\title{
Molecular Cloning of \\ Neuropathy Target Esterase
}

Thesis Submitted for the degree of

Doctor of Philosphy

at the University of Leicester

by

Michael John Lush

Department of Biochemistry

University of Leicester

Submitted February 1998 
UMI Number: U106116

All rights reserved

\section{INFORMATION TO ALL USERS}

The quality of this reproduction is dependent upon the quality of the copy submitted.

In the unlikely event that the author did not send a complete manuscript and there are missing pages, these will be noted. Also, if material had to be removed, a note will indicate the deletion.

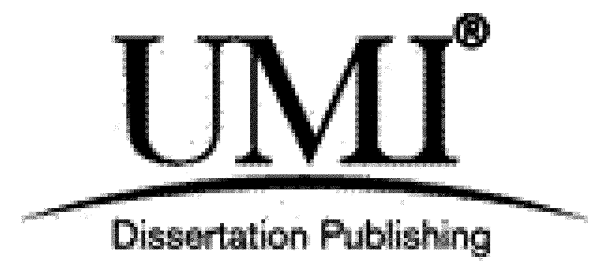

UMI U106116

Published by ProQuest LLC 2013. Copyright in the Dissertation held by the Author.

Microform Edition (c) ProQuest LLC.

All rights reserved. This work is protected against unauthorized copying under Title 17, United States Code.

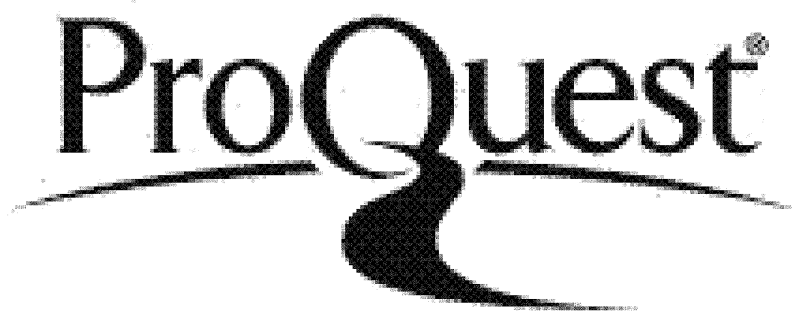

ProQuest LLC

789 East Eisenhower Parkway

P.O. Box 1346

Ann Arbor, MI 48106-1346 


\section{Acknowledgements}

I am deeply grateful to Dr Paul Glynn for his help, encouragement and advice during the course of this project and his guidance in the preparation of this thesis.

I would also like to thank Kath Lilley and the Protein and Nucleic Acid Chemistry Laboratory (Leicester University) for oligonucleotide synthesis, protein and DNA sequencing. I would also like to thank David Read for technical assistance and Mr and Mrs Lush for their support during the writing of this thesis.

Finally I indebted to Dr Ruth Akhtar, for her support over the last four years and especially for putting up with me while I wrote this thesis. 


\title{
Molecular Cloning of Neuropathy Target Esterase
}

\author{
Michael John Lush
}

\begin{abstract}
A single ingestion of certain organophosphorus esters (OPs) can cause a syndrome known as Organophosphate Induced Delayed Polyneuropathy (OPIDP), a paralysing neuropathy with degeneration of long axons, developing after a latent period of approximately one to three weeks. The primary target of these OPs has been shown to be a $155 \mathrm{kDa}$ neural protein designated Neuropathy Target Esterase (NTE), and the toxic effects apparently due to the covalent inhibition and subsequent secondary modification of this protein. Recently NTE has been purified to apparent homogeneity using a novel biotinylated $O P$ and sufficient pure protein was produced for limited protein sequencing.

The aim of this project was to clone NTE cDNA using peptide sequence data. Initially, these sequences were used to design degenerate oligonucleotide primers for amplifying sections of brain CDNA by polymerase chain reaction (PCR). These approaches were unsuccessful. Subsequently, database searching with the peptide sequences identified a number of Expressed Sequence Tags (ESTs); these could be aligned to form an initial contig of $2.2 \mathrm{kbp}$ which encoded the $3^{\prime}$ end of NTE cDNA. The 5' end of NTE CDNA, comprising a further $2.2 \mathrm{kbp}$, was obtained by a PCR-based technique. The final $4.4 \mathrm{kbp}$ contig encoded a 1327 residue polypeptide predicted to have a molecular mass of $146 \mathrm{kDa}$ and at least one transmembrane domain. A novel serine esterase domain of approximately 200 residues was present near the C-terminus. NTE is unrelated to any known serine hydrolases but homologous proteins are predicted to be present in diverse prokaryotic and eukaryotic organisms. The homologue in Drosophila is associated with the swisscheese phenotype, an age-dependent neurodegeneration of the brain. NTE was also mapped to chromosome 19p13.3 between markers D19216 and D19S413 (using the UniGene database) and an OMIM search reveals that this is near the locus of cerebellar ataxia (Cayman type).
\end{abstract}


$\begin{array}{lr}\text { Abstract } & \text { ii } \\ \text { Table of contents } & \text { ii } \\ \text { List of Figures } & \text { v } \\ \text { Abbreviations } & \text { ix }\end{array}$

\section{Chapter 1 Introduction}

$1.1 \quad$ Introduction

1.1.1 Organophosphate Induced Delayed Polyneuropathy 1

1.1.2 History of OPIDP 2

1.1.3 Organophosphate toxicology: acute effects 3

1.1.4 OPIDP is not an archetypal esterase inhibition 4 syndrome

1.1.5 Biochemistry of NTE

1.1.6 Candidate proteins for NTE 9

1.2 Introduction to cloning 11

1.2.1 Positional cloning 11

1.2.2 Functional selection 12

1.2.3 Immunochemical selection 13

1.2.4 Nucleic acid hybridisation selection 14

1.2.5 'Virtual' cloning 14

1.3 PCR cloning of NTE 16

1.3.1 Degenerate PCR 16

1.3.2 Rapid Amplification of CDNA Ends 18

$\begin{array}{lll}1.3 .3 & \text { 5' RACE } & 19\end{array}$

1.3.4 3' RACE 19

1.3.5 Other cloning work performed on NTE

\section{Chapter 2 Methods and reagents}

2.1 Protein methods 33

2.1.1 Preparation of microsomes 33

2.1.2 Protein Determination 34

2.1.3 S9B-labelling and solubilisation of microsomes 35

2.1.4 Solubilisation of microsomes and capture of NTE on avidin- 36 Sepharose

2.1.5 Analysis of prep-PAGE fractions 37

2.1.6 V8 protease digest 38

2.1.7 SDS-Polyacrylamide Gel Electrophoresis 39

2.2 DNA purification and cloning 41

2.2.1 Agarose gel electrophoresis 41

2.2.2 PCR product purification by electroelution 42

2.2.3 PCR Product purification by QIAspin kit 43

2.2.4 Phenol extraction and ethanol precipitation 44

2.2.5 Ethanol precipitation of oligonucelotides 45 
2.2.6 Purification of high molecular weight genomic DNA 45

2.2.7 DNA ligation with compatible sticky ends 48

2.2.8 pGEM-T cloning of PCR products 48

2.2.9 pCRscript cloning of PCR products 49

2.2.10 Transformation of ligated products 50

2.3 PCR Methods 52

2.3.1 General PCR and RACE methods 52

2.3.2 Direct PCR of bacterial colonies 53

2.3.2 Cycle sequencing 54

2.3.3 Preparation of a circular genomic DNA library for inverse 55 PCR

2.3.4 Synthesis of CDNA for 3' RACE and RT-PCR 56

2.3.6 DEPC treatment of reagents and equipment 57

2.3.6 Amplification of CDNA bacteriophage library for use as 58 PCR template

2.4 Hybridisation screening 60

2.4.1 Transfer of bacterial or bacteriophage colonies to solid 60 support

2.4.2 Southern Blotting 61

2.4.3 Labeling DNA probes 62

2.2.4 Hybridisation conditions 63

\section{Chapter 3 Isolation and protein sequencing of NTE}

3.1 Introduction 64

3.2 Results and Discussion 65

3.2.1 Chicken brain NTE: Purification and protein sequencing 65

3.2.2 Pig brain NTE: Purification and protein sequencing 67

3.3 Significance of NTE peptide sequences in isolating the 68 NTE CDNA

Chapter 4 Attempts to clone NTE cDNA using Rapid Amplification of CDNA Ends (RACE)

4.1 Introduction 81

4.2 Results and Discussion 81

4.2.1 Synthesis and quality control of CDNA 81

4.2.2 Ratification of 3' RACE protocol 82

4.2.3 3' RACE with degenerate primers designed to NTE 83 peptides

4.2.4 Nested 3' RACE $\quad 84$

$\begin{array}{lll}4.3 & \text { Perspectives on Degenerate 3' RACE } & 87\end{array}$ 


\section{Chapter 5 Attempts to clone NTE cDNA by PCR using degenerate primers of inferred relative position}
5.1
Introduction
100
5.2 Results and discussion
100
5.2.1 Production and isolation of PCR-generated clone ORF1 100
5.2.2 Attempts to clone the ORF1 template 103
5.3 Retrospective view on the relative positions of LTNP, 105 XGVP and the catalytic serine in NTE

\section{Chapter 6 Cloning of NTE via homology to an expressed} sequence tag
6.1 Introduction
6.2 Results and discussion
120
6.2.1 Sequencing the 3' end of NTE CDNA by alignment of ESTs
120
6.2.2 Subcloning of the $2 \mathrm{kbp}$ insert from pDR2 into pBluescript SK+
6.2.3 Cloning of the 5 ' end of NTE by 5' RACE
6.2.4 Sequencing of the 5' RACE products 124
6.3 Review of gene cloning

Chapter 7 Analysis of the NTE CDNA and deduced protein sequence

$\begin{array}{lll}7.1 & \text { Introduction } & 137\end{array}$

7.2 Results and Discussion 137

7.2.1 The NTE1 clones produced by the 5' RACE contain 11 out 137 of 12 of the NTE peptide sequences.

7.2.2 NTE shares a putative novel esterase domain with proteins 139 from both eukaryotes and prokaryotes.

7.2.3 Function of the N-terminal domain of NTE 140

7.2.4 Computer analyses of NTE and YOL4 sequences indicate 142 NTE is a membrane bound protein.

7.2.5 Mutation to the swisscheese (sws) gene can produce a 143 neurodegeneration syndrome or a specific anosmia to aldehydes (olfE).

7.2.6 The NTE gene is located on chromosome 19p13.3 near the 144 mapped position of a recessive heritable ataxia.

$\begin{array}{lll}7.3 & \text { Overview of predictions } & 147\end{array}$

$\begin{array}{lll}7.4 & \text { Future work } & 148\end{array}$

7.4.1 What is the membrane topology of NTE and what 148 organelles is it associated with?

7.4.2 What are the biological functions of the various domains of 149 the NTE protein?

7.4.3 What is the biological role of NTE and what is its role in the 151

7.5 Conclusion 


\section{List of Figures}

Figure 1.1

General structure of organophosphorus esters

Figure 1.2

Catalytic mechanism of serine esterases.

Figure 1.3

Figure 1.4 Mechanism esterases.

Figure 1.5 Prediction of OP delayed neuropathic potential.

Figure 1.6

Figure 1.7 Tissue distribution of NTE activity as a percentage of adult hen brain NTE activity (reproduced from Johnson, 1992).

Figure 1.8 General structure of the biotinylated saligenin phosphoramidates used to purify NTE.

Figure 1.9 AChE-like enzymes which lack catalytic activity.

Figure 1.10 Synthesis of CDNA for EST sequencing produces a common $3^{\prime}$ end sequence and many 5 ' end sequences from the same gene.

Figure 1.11 Amino acid degeneracy codes.

Figure 1.12 Synthesis of CDNA for 3'RACE.

Figure 3.1 Structure of 1, [Saligenin cyclic phosphoryl],-9,- 71 biotinyl-diaminonoane (S9B).

Figure 3.2 Flow chart summarising the purification stages of NTE 72 from chicken brain to purified NTE.

Figure $3.37 .5 \%$ SDS-PAGE illustrating the two stages of NTE 73 purification.

Figure 3.4 7.5\% SDS-PAGE of aliquots from Preparative SDSPAGE of Avidin eluate.

Figure 3.5 V8-digested NTE blotted on to Immobilon P prior to sequencing.

Figure 3.6 Chicken brain peptide sequence. containing the S9B binding site. 
Figure 3.9 Peptide sequence derived from HPLC purified tryptic digest fragments from V8 digest fragments of pig NTE.

Figure 3.10 Sequence similarities between ADLT, WPG and sequences linked to YOL4.

Figure 4.1 Pig NTE peptide sequence available for degenerate primer design.

Figure 4.2 Design of anchor primers for reverse transcription of poly-A RNA and 3' RACE.

Figure 4.3 Relative positions of trk1+, trk3-, trk5+ and trk9+

92 primers on pig trkC CDNA.

Figure 4.4 Quality control of BAT39-primed CDNA for 3' RACE 93

Figure 4.5 Ratification of 3' RACE protocol.

94

Figure 4.6 Design of degenerate primers from NTE peptides for

95 3' RACE experiment.

Figure 4.7 3' RACE with the F10I degenerate primer.

Figure 4.8 Translations of the DNA sequences from the $5^{\prime}$ (F10I) end of 3' RACE products.

Figure 4.9 PCR amplification of BAT39-primed CDNA with degenerate primers F36IS, F36IT, F16I, F13IG and F13IY paired with reverse primers BAT117 and BAT318.

Figure 4.10 Typical example of 2nd round nested 3' RACE with degenerate primers.

Figure 5.1 Putative peptide map of the serine esterase domain of 107 NTE.

Figure 5.2 Design of degenerate primers to the LTNP and XGVP 108 peptide sequences

Figure 5.3 PCR amplification of oligo-dT primed pig brain CDNA 109 using primers of putatively known position.

Figure 5.4 Translation of ORF1 and ORF2 sequence in three reading frames.

98

Figure 5.5 Alignment ORF1 with the N-termini of multiple serine esterase domains.

Figure 5.6 ORFA, ORFD, ORFE and ORRA primer locations on 112 ORF1 sequence.

Figure 5.7 Inferred consensus sequence flanking the catalytic serine in NTE.

Figure 5.8 Inverse PCR allows amplification of DNA sequences 114 flanking a small section of known sequence.

Figure 5.9 Inverse PCR on Mbol circularised pig genomic DNA with non-degenerate primers ORFA, ORFD and ORRA.

Figure 5.10 Alignment of ORF1 templates

Figure 5.11 Peptide map around the S9B-labelled serine of NTE, using sequence data from Figure 7.1. 
Figure 6.1 Similarity between the pig NTE peptide sequence

LAKL and YOL4 CAEEL, LAKL and RUBISCO,

DLGL and YOL4_CAEEL, DLGL and Acetyl-CoA carboxylase.

Figure 6.2 Alignment between YCHK_ECOLI, YCHK_SHFTL, and the C-terminal domain of YOL4 CAEEL.

Figure 6.3 Similarity between translated EST sequences and the 128 DLGL, DGHL, WKS and FDQI peptide sequences.

Figure 6.4 Relative positions of primers in insert and pDR2 vector sequence at the 5 ' end of the HOL4.2 insert.

Figure 6.5 PCR of DRA/B primers vs NDR2/3/4 on IDR2 adult human brain CDNA library.

Figure 6.6 Synthesis of Marathon CDNA.

Figure 6.7 PCR amplification using NDR2 and NDR4 vs AP1 primers on Marathon CDNA.

Figure 6.8 PCR with RSP vs NDR4 sizing the clones.

Figure 6.95 ' end sequence from the NTE1 and NTE2 product

Figure 6.10 groups.

Figure 6.11

Overlap between the NTE1 clone and HOL4.2 CDNA Detection of the initiation codon of the NTE1 CDNA and alternate splice form NTE2 of the 5 ' end of the putative CDNA.

Figure 7.1 Positions of the known pig NTE peptide sequences on the sequence of human NTE cDNA.

Figure 7.2 Summary of the hypothetical proteins with a similarity to NTE's C-terminal esterase domain.

Figure 7.3 Comparison of NTE like proteins.

Figure 7.4 Multiple alignment of the putative esterase domain of 156 proteins in Figure 7.3.

Figure 7.5 Similarity plot of data in Figure 7.4

Figure 7.6 Dotplot of YMF9 (vertical) vs NTE (horizontal) 158

Figure 7.7 Dotplot of NTE (horizontal) vs sws (A) and YOL4 (B). 159

Figure 7.8 Transmembrane predictions for NTE. 160

Figure 7.9 Putative secondary structure of NTE (not to scale). 161

Figure 7.10 Cytogenetic map of Chromosome 19 and genetic map 162 of the ATCAY/NTE location. 


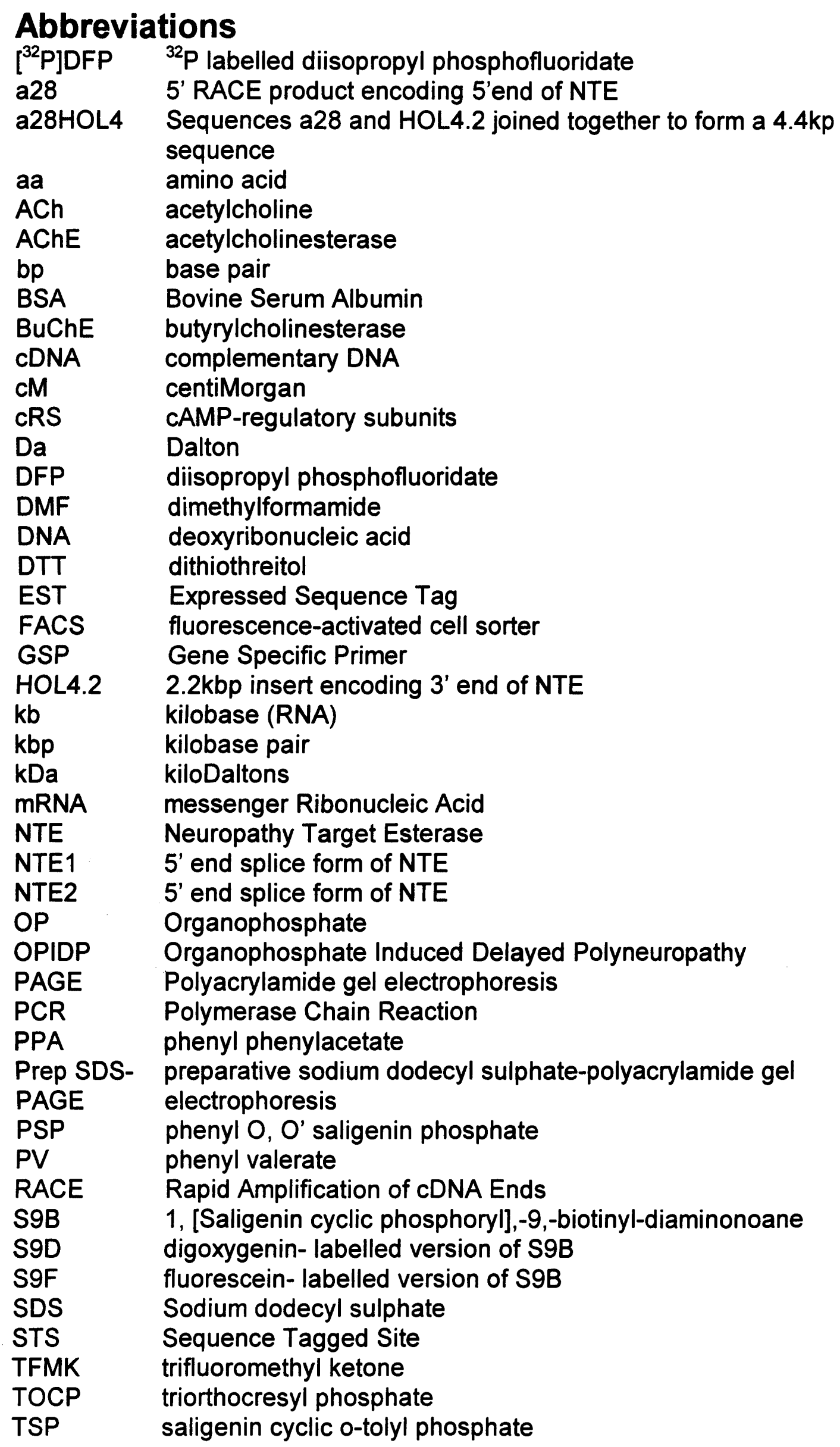




\section{Chapter 1 Introduction}

\subsection{Introduction}

\subsubsection{Organophosphate Induced Delayed Polyneuropathy}

Organophosphate Induced Delayed Polyneuropathy (OPIDP) is a syndrome caused by certain organophosphate (OP) compounds and is characterised by a flaccid paralysis of the lower limbs developing 1 to 3 weeks after a single dose of OP. Clinically, OPIDP presents as initial sensory disorders (cramp and tingling), followed by weakness and ataxia in the lower extremities progressing to a flaccid paralysis; in severe cases upper limbs can also be affected. The symptoms are relatively permanent although some recovery is possible as peripheral nerves repair (Lotti, 1992).

Histological study of the damaged nerves shows the initial leason manifesting at the same time as clinical signs (ie after 1 to 3 weeks), and expressed as a localised degeneration at the distal (though not terminal) end of individual nerve fibres. Large and long fibres (ie, those in the peripheral nerves and spinal cord) are the most susceptible to damage and as the syndrome progresses, an increasing proportion of the axons become involved and the already damaged axons undergo degeneration progressing up the axon but not affecting the cell body. Demyelination is also observed though this appears to be a secondary effect caused by the neuropathy (Bouldin and Cavanagh, 1979).

OPIDP is interesting from two view points: Firstly, study of the protein target of delayed neuropathic OPs has allowed the development of an in vitro testing system for the delayed neuropathic potential of many compounds; (this is important practically because hundreds of different OPs have been synthesised as candidate pesticides). Secondly, study of this syndrome has the potential to 
elucidate part of the mechanism of axonal maintenance which is critically disrupted during OPIDP.

At the outset of this project, the protein target of the neuropathic OP, Neuropathy Target Esterase (NTE) had recently been isolated in this laboratory. The goal of this project was to use the purified NTE as a starting point to clone its CDNA. Possession of an NTE clone is an attractive proposition since, despite more than 60 years of research, almost all the data relating to NTE and OPIDP is essentially based on the inhibition of esterase activity of NTE, which is known to be unrelated to the actual genesis of the neuropathy (see Section 1.1.5). Therefore possession of NTE's DNA (and hence protein) sequence, would allow a number of approaches to the study of NTE, which have been hitherto impossible:

- Synthetic peptides or expressed NTE protein could be used to raise high titer antisera, making it possible to visualise NTE at a cellular level.

- NTE's protein sequence could be compared with that of already characterised proteins with similar sequence and might, in itself provide clues as to NTEs function.

- Expression, biochemical (and subsequently structural) study of catalytically active NTE would become feasible, since until now it has only proved possible to purify heavily denatured and inhibited NTE.

\subsubsection{History of OPIDP}

The earliest reported cases of OPIDP occurred during 1899 in France, as a result of using a phospho-creosote mixture in the treatment of pulmonarytuberculosis, although it was not till the 1930s that it OPIDP was recognised as a syndrome (Inoue et al, 1988).

In the spring of 1930 in the midwestern and south-western states of the United States of America, an outbreak of a what is now known as OPIDP affected as many as 50,000 people (Inoue et al, 1988). The polyneuropathy was 
eventually traced by Smith et al, (1930) to a popular drink called 'Ginger Jake' (an $80 \%$ alcoholic extract of Jamaica ginger, sold as a medical tonic to evade the Prohibition laws) which had been adulterated by the addition of approximately $2 \%$ triorthocresyl phosphate (TOCP). A 47 year follow up study on eleven survivors of the Ginger Jake poisoning still showed signs of abnormal reflexes, paralysis and spasticity (Morgan and Penoich, 1978). Another notable outbreak of OPIDP happened during 1959 in Morocco when 10,000 people were poisoned with cooking oil adulterated with jet engine oil (Inoue et al, 1988). In 1953 three research workers developed OPIDP after working on new insecticide called mipafox (Bidstrup et al, 1953) these were the first reported cases of OPIDP caused by compounds other than TOCP; since that time the list of OPIDP causing OPs has grown, thanks, in no small part to the routine screening of all new OP compounds for neuropathic potential.

\subsubsection{Organophosphate toxicology: acute effects}

Organophosphates are widely used as pesticides and also have limited medical applications (Figure 1.1). The acute neurotoxic action of many OPs is almost entirely due to their inactivation of acetylcholinesterase (AChE) by covalent reaction with its catalytic site. $\mathrm{AChE}$ is an important part of the nervous system, as it is responsible for inactivating the neurotransmitter acetylcholine (ACh) in the synapses of the brain and neuromuscular junction. Inhibition of AChE leads to a build up of the stimulatory $A C h$ in the synapse leading to continual stimulation of the connecting neuron or muscle, ultimately causing paralysis and death by respiratory failure.

The hydrolytic mechanism of AChE utilises a charge relay system of a serine, histidine and aspartate to activate the serine and enable it to make a nucleophilic attack on the carbonyl group of the (non-covalently) bound substrate (Figure 1.2). The inhibitory power of the toxic OPs is partly due to the tetrahedral structure of the phosphate group, since it resembles the tetrahedral transition state Michaelis complex of the reacting ACh molecule (Figure 1.2). The other 
factor in their toxicity is the permanence of the covalent linkage to the catalytic serine. This is dependent on the structure of the inhibiting OP: for example, a dimethyl phosphate adduct is removed by spontaneous hydrolysis in a matter of hours; in contrast inhibition by a diisopropyl phosphate group is essentially irreversible. The reason for this is a phenomenon known as 'ageing' in which a single group is spontaneously hydrolysed from the phosphate rendering the inhibition irreversible (Figure 1.3; Johnson, 1992).

\subsubsection{OPIDP is not an archetypal esterase inhibition syndrome}

The clinical syndrome known as Organophosphate Induced Delayed Polyneuropathy represents an unusual response to challenge by toxic OP compounds. Rather than an immediate onset of symptoms as is displayed in response to anti-AChE compounds, there is an asymptomatic period of 1 to 3 weeks prior to the development of the peripheral neuropathy

Originally, W. N. Aldridge (1954) suggested that, since the neuropathic agent was an OP, then the likely initiating stage in OPIDP would involve the OP phosphorylation of an esterase (now called Neuropathy Target Esterase; NTE). Initial theories posited inhibition of brain AChE or butyrylcholinesterase (BuChE), however these theories had to be discarded due to the existence of OPs which caused OPIDP but did not inhibit the suggested esterase activities (Abou-Donia, 1981).

TOCP is relatively inert compound (perhaps explaining why it was selected as an adulterant for Ginger Jake) and does not inhibit NTE in vitro. It requires bioactivation to saligenin cyclic o-tolyl phosphate (TSP; Figure 1.4) to both inhibit NTE and trigger OPIDP (Casida et al, 1961; Eto et al, 1962). This prompted the observation from Poulsen and Aldridge (1964) that there was a certain structural similarity between TSP, phenyl phenylacetate (PPA) and that the latter could be a substrate for NTE. 
By screening for compounds that halted progressive labelling of the neuropathy target site by $\left[{ }^{32} \mathrm{P}\right]$ diisopropyl phosphofluoridate $\left({ }^{32} \mathrm{P}\right] \mathrm{DFP}$; an OP with both anti-AChE activity and also a delayed neuropathic effect (Johnson, 1969b), it was possible to dissect the various enzyme activities by differential inhibition using non-neuropathic and neuropathic compounds. Paroxon a nonneuropathic OP was used to eliminate much of the non-NTE esterase activity; this left about $9-11 \%$ PPA esterase or $\left[{ }^{32}\right.$ P]DFP labelling activity. The addition of mipafox (a potent neuropathic OP; Bidstrup et al, 1953) resulted in a further 3$4 \%$ loss of activity. This $3-4 \%$ PPA activity insensitive to paraoxon but sensitive to mipafox is the functional definition of NTE. Definitive assay conditions for NTE assay were laid down by Johnson (1978); the main alteration to the protocol was that substrate (PPA) was replaced by phenyl valerate (PV). The PV NTE assay has been successful in predicting the delayed neuropathic potential of more than 100 OPs and at least 20 organophosphinates, sulphonyl fluorides and carbamates (Johnson, 1975a and 1982). However, to achieve this level of success it is necessary to consider not only the inhibition of NTE but also the structure of the toxin.

As with OP inhibition of AChE, the OP group bound to NTE is faced with two possible fates; slow spontaneous hydrolysis from the enzyme, or ageing by loss of one of the residual groups from the phosphorus, rendering the inhibition irreversible and also introducing a negative charge to the active site serine (Figure 1.3). Curiously, unlike AChE, simple inhibition of NTE esterase activity, even for a period of weeks, does not necessarily lead to OPIDP (Johnson, 1970). It is clear that the non-neuropathic NTE inhibitors attack the same site in the enzyme as these reagents have a prophylactic effect preventing the action of neuropathic OPs by physically blocking the catalytic cleft. By examining a compound's ability to age and its activity against NTE it is possible to predict the neuropathic potential of an OP compound (Figure 1.5).

One particularly elegant demonstration of the necessity for ageing to trigger OPIDP was produced by Johnson and Read (1987). EPNO (Figure 1.1) is 
an inhibitor of NTE and in vitro the potency of the $L(-)$ and $D(+)$ isomers are similar; however the $L(-)$ isomer is rapidly aged in NTE whereas the $D(+)$ isomer does not age, despite the similar structure and presence of a potentially cleavable R-O-P bond. When tested in vivo the $L(-)$ isomer did induce OPIDP whereas the $D(+)$ isomer was not only non-neuropathic, but also prophylactic if administered prior to a dose of phenyl $O, O^{\prime}$ saligenin phosphate (PSP; another member of the highly neuropathic saligenin phosphate family of NTE inhibitors; Figure 1.1).

Another of the interesting features of the ageing reaction in NTE, compared to that in AChE is that the leaving isopropyl moiety from DFP is retained within the enzyme with $100 \%$ efficiency (Clothier and Johnson, 1979; Williams and Johnson, 1981). This intramolecular transfer is not observed with all neuropathic OPs and thus is not critical for the initiation of the delayed neuropathy. For example, the highly neurotoxic octyl saligenin cyclic phosphate was found to age but does not display a similar intramolecular group transfer (Yoshida et al, 1995) indicating that it is the introduction of a negatively charged group at the catalytic site that initiates OPIDP.

The initiating events of OPIDP are well known: about 70 to $80 \%$ of NTE activity needs to be inhibited to trigger neuropathy and the inhibiting $O P$ is aged within a few minutes (Clothier and Johnson, 1974); after this stage the actual physiological disturbance set up by the inhibition is unknown. What is known is that NTE has a half life of reappearance of 2 days in brain and 3.6 days in spinal cord after inhibition with a neuropathic OP (Meridith and Johnson, 1988). Thus NTE activity is completely restored by the time paralysis develops and it may be the disruption of NTE's function for a limited period that causes a knock-on effect on the stability of the axonal function. In addition there appears to be inhibition of retrograde axonal transport (of injected ${ }^{125}$-labelled tetanus toxin) at five to seven days after OP challenge, although whether this represents cause or effect is unknown (Moretto et al, 1987). 


\subsubsection{Biochemistry of NTE}

The molecular mass of denatured NTE was established to be approximately $155 \mathrm{kDa}$ by Williams and Johnson (1981); this was determined by $\left[H^{3}\right]$ DFP-labelling of brain microsomes which had been pre-treated with paraoxon or paraoxon+mipafox, followed by solubilisation and SDS-PAGE. Various attempts have been made to measure the mass of catalytically active NTE by gel filtration: estimates have ranged from $1800 \mathrm{kDa}$ (Chemnitius et al, 1984) through 970kDa (Pope and Padilla, 1989) to 850kDa (Thomas et al, 1990). The discrepancy in size between the native and denatured forms of NTE is probably because NTE forms an aggregate which is apparently necessary for its activity. Pope and Padilla (1989) reported that their 970kDa fraction contained a 148 to $160 \mathrm{kDa}$ polypeptide which possessed the appropriate $\left[{ }^{3} \mathrm{H}\right] \mathrm{DFP}$ binding characteristics for NTE.

Besides its size, little is known about NTE. It is a glycoprotein (P. Glynn, unpublished data) and it can hydrolyse several artificial ester substrates but appears inactive against several peptide substrates (Johnson, 1975b). However, its physiological substrate is unknown and it is even possible that it has no physiological substrate, especially since the near complete inhibition of NTE for a period of weeks appears to have no obvious adverse effect in adult chickens (Johnson, 1970). Figure 1.6 summarises the tissue distribution of NTE in the adult hen. It is worth noting that, even though NTE is present in non-neural tissue, the toxic effects of neuropathic OPs appear to be restricted to long axons in the spinal cord and peripheral nerves (Bouldin and Cavanagh, 1979).

Detergent solubilization studies show NTE is firmly associated with microsomal membranes and quite hydrophobic in nature (Davis and Richardson, 1987). Recent immunolocalisation studies have shown that NTE is located in neurons but not glia and the staining pattern indicates that NTE is located intracellularly, but not in the nucleus (Glynn et al, 1998). Carrington and AbouDonia (1985) have shown that NTE undergoes fast axonal transport. These 
findings are supported by immunohistochemical staining of NTE showing that it accumulates at a ligature on the sciatic nerve, mainly on the anterograde side and to a lesser extent the retrograde side of the blockage with staining building up after a period of 6 to 8 hours. The axonal transport of NTE is apparently independent of its modification by neuropathic OPs, as dosing chickens with a neuropathic OP has no gross qualitative or quantitative effects on the accumulation of NTE at the nerve ligature (Glynn et al, 1998).

A prerequisite for the goal of this project (ie, the cloning of NTE) is the ability to isolate the pure protein. However for many years NTE resisted purification attempts in several laboratories. This was primarily due to its low abundance ([ $\left.\mathrm{H}^{3}\right]$ DFP labelling indicates that NTE comprises only $0.03 \%$ of brain microsomal protein; Williams and Johnson, 1981), and poor recovery of detergent solubilized $\left[\mathrm{H}^{3}\right.$ ]DFP labelled NTE by conventional purification techniques such as ion-exchange and hydrophobic interaction chromatography gel filtration and sucrose gradient centrifugation. (Thomas et al, 1993). NTE activity is highly labile to even simple procedures such as dialysis and ultrafiltration, probably due to loss by surface adsorption (Pope and Padilla, 1989).

Previously the best purification protocol utilised Nonidet P40 solubilization of $\left[^{3} \mathrm{H}\right] \mathrm{DFP}$-labelled brain microsomes, followed by detergent phase partitioning, anion exchange and preparative sodium dodecyl sulphate-polyacrylamide gel electrophoresis (Prep SDS-PAGE). This protocol yielded a fraction in which NTE comprised only $2.5 \%$ of the total protein (Rüffer-Turner et al, 1992). Sequential proteolytic digestion and purification stages on the enriched NTE fraction yielded a seven residue heterogeneous sequence within which the serine esterase consensus sequence could be discerned (Figure 1.7; Glynn et al, 1993).

The next generation of NTE purification attempts focused on using NTE inhibitors to bind and immobilise the NTE so it could be purified by affinity chromatography. One approach used a trifluoromethyl ketone (TFMK) liganded 
onto Sepharose CL6B; the TFMK used (3-(9'-mercaptononylthio)-1,1,1trifluoropropan-2-one) is a reversible inhibitor for a wide range of serine hydrolases including NTE, and when used as an affinity ligand it was moderately successful in purifying NTE (Thomaes et al, 1993). However the technique was apparently not sufficient to purify NTE on a preparative scale as a later paper from the same research group did not report any $\mathrm{N}$-terminal sequence data (Mackay et al, 1996)

The approach used in this project was to use one of the saligenin cyclic phosphoryl OPs (Figure 1.1). A series of novel biotinylated saligenin phosphoramidates which differed only by the length of the alkyldiamine spacer were synthesised (Figure 1.8) and their inhibitory potency assessed in the PV assay for NTE (see Section 1.1.4). The diaminonoane derivative (1, [Saligenin cyclic phosphoryl],-9,-biotinyl-diaminonoane (S9B)) was one of the two most potent inhibitors and was adopted as the main purification reagent (Glynn et al, 1994).

S9B proved to be able to specifically biotinylate NTE in a complex mixture of microsomal protein. A simple absorption/washing/elution of the biotinyl-NTE complex bound to an avidin-Sepharose affinity column allowed a 1000 fold enrichment of NTE with recoveries of $30 \%$. A subsequent Preparative SDS-PAGE step removed contaminating endogenously biotinylated protein and yielded essentially pure NTE protein (Glynn et al, 1994). Chapter 3 describes purification of chicken and pig brain NTE, its digestion and $\mathrm{N}$-terminal sequencing.

\subsubsection{Candidate proteins for NTE}

At the outset of the project it was thought that NTE was likely to be a member of the AChE family of serine esterases, which included enzymes capable of catalysing the hydrolysis of a wide variety of ester substrates (Krejci et al, 1991). Additionally there was a growing body of research showing that the AChE protein had significant non-catalytic function. Studies using antibodies 
capable of discriminating between active and inactive $\mathrm{AChE}$ shows that $30 \%$ or the total AChE protein is inactive, which indicates a large pool of enzyme apparently uninvolved in its supposed primary physiological role (Chatel et al, 1993). Furthermore, inhibition of $A C h E$ can lead to a dose dependent decrease in chick neurite growth in vitro; however simple inhibition of the AChE appears to be insufficient to cause this effect since there is at least one AChE inhibitor which does not have this effect (Layer et al, 1993).

One group of proteins (neurotactin, glutactin, gliotactin and neuroligins 1 to 3; see Figure 1.9) within the AChE family are of particular interest. All are large glycoproteins which possess a domain with a significant sequence similarity to AChE, yet are enzymically inactive since they lack a catalytic serine. These enzymes appear to be involved in cell-cell interactions mediated through the extracelluar AChE-like domain. Since the proteins have a comparable size and like NTE do not require catalytic activity for function, it was thought that NTE might be a new member of this protein family in which the catalytic serine had been retained, allowing NTE to keep a vestigial esterase activity unrelated to its physiological function. In this model inhibition and ageing of the AChE-like domain would disrupt the protein-protein interactions by which cells communicated. This line of thinking had a considerable influence on certain decisions taken during this project, particularly at the stages described in Chapters 5 and 6 


\subsection{Introduction to cloning}

Gene cloning projects essentially rely on the selection of a DNA clone from a library of clones which represent all or most of the genes in a organism or a particular tissue or cell type.

Selection strategies for cloning an animal gene fall into 5 broad categories:-

\subsubsection{Positional cloning}

\subsubsection{Functional selection}

\subsubsection{Immunochemical selection}

\subsubsection{Nucleic acid hybridisation selection}

\subsection{5 'Virtual' cloning}

\subsubsection{Positional cloning}

It is possible to locate and clone genes responsible for genetic diseases simply on the basis of their position within the genome. Unless the disease is caused by large scale chromosomal rearrangements which are cytogenetically detectable, the gene localisation usually takes the form of linkage analysis (Wicking and Willamson, 1991).

Linkage analysis works by studying the way a disease or trait is inherited relative to DNA markers of known chromosomal location. Once the chromosomal location of the trait is determined, normally an interval of about 100 to $1000 \mathrm{kbp}$, it is then necessary to clone and search the genomic DNA for gene coding regions and, by a process of elimination, isolate the disease gene (Wicking and Willamson, 1991). However, since OPIDP does not closely resemble any known genetic disease and no strain polymorphisms are known which could be used to track NTE, linkage analysis is completely unsuitable as a possible cloning approach. 


\subsubsection{Functional selection}

Functional selection exploits the biochemical properties of a protein to isolate the gene. Messenger RNA (mRNA) is isolated from the organ known to express the desired protein. It is then reverse transcribed to make CDNA and cloned into a plasmid with suitable promoter for expression in the vector's host. In theory, a cDNA expression library could be screened for the binding of S9B. However, there were a number of drawbacks to this approach. Due to NTE's large size and consequent high complexity, it seems unlikely that NTE would express as an active protein in a bacterial or yeast expression system, necessitating the use of more technically-challenging hosts such as insect or mammalian cell lines. Furthermore, since biotin is an endogenous cellular component it would probably cause an unacceptably-high background if a biotinlabelled probe was used to screen the library. Thus, a fluorescein- or digoxygenin-labelled version of S9B (S9F and S9D, respectively) would need to be synthesised.

One possible benefit to using a fluorescein-labelled saligenin phosphoramidate would be the ability to use a fluorescence-activated cell sorter (FACS) to screen and separate individual cells on the basis of S9F binding. This approach was used by Rice et al (1990) to clone a full length CD4 CDNA in a COS-7 host cell by screening 500,000 independent clones. FACS sorting of NTE would probably be a rather more demanding proposition. Rice et al already had a CD4 CDNA with which they had developed the system. Furthermore, CD4 is a cell surface protein and was detected by labelled antibody. Labelled saligenin phosphates are very hydrophobic and would permeate the entire cell with the S9F probably lodging in the cell membranes, leading to a high fluorescent background possibly masking any NTE signal.

The use of the traditional PV assay for NTE, with differential inhibition by paraoxon and mipafox (Section 1.1.4) would be too cumbersome and probably 
too insensitive for this approach. Thus, functional cloning of NTE was not pursued as part of the cloning strategy.

\subsubsection{Immunochemical selection}

In antibody selection, cDNA is cloned into an expression vector and transferred into either a bacterial or bacteriophage host strain. The recombinant micro-organisms are grown to form colonies, then are transferred onto a suitable solid support (traditionally nitro-cellulose membrane). The immobilised colonies are then screened for binding of an antibody specific to the desired protein.

Antibody selection has the advantage over functional selection in that the protein need not be biochemically active to be detected. However, as with functional selection, the cDNA can be of different lengths (due to the reverse transcriptase failing to completely copy the mRNA). The cDNA is thus inserted into the vector in a random reading frame resulting in two thirds of the clones expressing in the wrong reading frame, effectively rendering them invisible to the antibody. Nevertheless, this approach has been successfully used to isolate over 200 genes and was not lightly dismissed (Sambrook et al, 1989b).

Rabbit antisera raised against a synthetic peptide designed from chicken brain NTE sequence provided the possibility of screening an expression library. However, these antisera were relatively low titre and on Western blots of microsomal protein stained not only NTE but several other polypeptides. Some experiments were performed to test the feasibility of antibody screening on colony lifts from a $\lambda Z A P$ CDNA library. This showed that the serum had a significant amount of background reactivity to the lysed $E$. coli proteins present in the plaques. This background could not be satisfactorily removed by preabsorption with a lysed $E$. coli preparation and antibody selection was not pursued further. 


\subsubsection{Nucleic acid hybridisation selection}

Nucleic acid hybridisation selection exploits the specific base pairing between complementary DNA strands. Conventionally this can be used in two ways:-

A) Hybridisation screening of a DNA library. In which a radioactively- or fluorescently- labelled DNA probe is used to identify clones in a DNA library with matching sequence (Sambrook et al, 1989b).

B) Polymerase chain reaction (PCR) in which two relatively short oligonucleotides are used to amplify regions of intervening DNA (Taylor, 1991).

Prior to the initiation of the project a commercial Clontech pig brain cDNA library was screened using two degenerate oligonucleotide probes designed using pig brain NTE sequence. This experiment failed to produce any positive clones, so a PCR based cloning strategy was pursued; this is discussed in Section 1.3.5.

\subsection{5 'Virtual' cloning}

One particularly attractive approach to cloning any gene is by searching the genetic databases for sequences similar to fragments of protein sequence determined by Edman degradation. There are two databases of particular relevance to gene cloning: EMBL and SWISSPROT databases.

The SWISSPROT protein database, though smaller than EMBL (the current releases have around 70,000 and 500,000 entries respectively), is more thoroughly annotated and cross-referenced. This makes it suitable for initial screening of peptide sequence to determine if the sequence is novel and also search for proteins which are similar to two or more NTE sequences. Discovery of such a protein would be useful to the project as it could provide a guide to the 
relative positions of the peptide sequences and possibly provide clues as to NTE's function.

Although other genome databases are available (GenBank and DNA database of Japan) the exchange of data between them render them effectively identical and since EMBL was available on the local computing resources (Leicester University Computing Service and the Hixtons Human Genome Mapping Project computers) EMBL was used as the default database for almost all searches.

EMBL is a DNA database and contains DNA sequence from a variety of sources. Besides the cDNA sequences for already cloned genes, it also contains genomic sequence from the many genome sequencing projects currently in progress. Of particular interest is the Expressed Sequence Tag (EST) division of EMBL. ESTs are the result of picking and sequencing random clones from a directional CDNA library, often one that has been 'normalised', i.e. one that is synthesised to reduce the representation of highly expressed genes (Schuler et al, 1996). Each EST clone is sequenced once at the 5' end and once at the 3' end using standard primers flanking the plasmid's cloning site. Each sequencing run normally yields between 300-500 bp of DNA sequence. The clones are then propagated and usually made available via the IMAGE consortium (Lennon et al, 1996). Therefore any matches between a peptide sequence and an EST can be easily investigated either by obtaining the clone from IMAGE or by further research through the database.

One useful feature of the EST sequences is the asymmetry of the $5^{\prime}$ and $3^{\prime}$ end sequence results. Because the cDNA library was made using an oligo-dT primer, the overwhelming majority of 3' end sequences initiate from the very end of the $3^{\prime}$ untranslated region and almost all of the 3 ' end sequences record the same data. Conversely the initiation point for the $5^{\prime}$ end data is essentially random, determined by the termination of the reverse transcriptase. The 3' end sequence is useful to cluster ESTs by transcript and identify a group of 
corresponding 5 ' end sequences even if these have no overlap (see Figure 1.10). This feature of EST is used in the UniGene project (Schuler et al, 1996) to produce Sequence Tagged Site markers (discussed in Section 7.2.6).

\subsection{PCR cloning of NTE}

\subsubsection{Degenerate PCR}

In conventional PCR, a unique pair of oligonucleotides are designed using known DNA sequence. These are used to amplify the region of DNA lying between them, normally producing a product of predictable size and often allowing the identification of the correct product by agarose gel electrophoresis.

When only peptide sequence is available, it is possible to deduce the DNA sequence which encodes the peptide sequence. However, due to the degeneracy of the genetic code a single amino acid can be coded for by up to six different codons (Figure 1.11). Thus, rather than synthesising a single oligonucleotide primer, a mixture of oligonucleotide primers are synthesised, each primer in the mix representing one possible permutation of DNA sequence which encodes the gene-specific peptide sequence. This degenerate sequence PCR can be used as an approach to gene cloning (McPherson et al, 1991).

An important consideration in designing degenerate primers is to keep the number of primers in the pool (the degeneracy) to a minimum. Since, as the degeneracy increases the proportion of primer identical to the desired gene drops, reducing the amount of product that can be produced as there is less

primer to produce it. Secondly and more significantly, as the degeneracy increases, so does the number of primers that do not match the desired product; thus the chance of spurious product production increases, which can easily swamp any genuine signal. 
The NTE peptide sequences available at the initiation of cloning of pig cDNA were assessed for reliability of the peptide sequence and their suitability for degenerate primer design (Figure 4.1). As a basis for a gene cloning project the sequences were less than optimal. Either the sequences were short, resulting in a short degenerate primer with a low melting temperature and high chance that the sequence is not unique in the genome, resulting in false positives. Alternately, the longer sequences contained the highly degenerate amino acids leucine, arginine or serine which radically increase the degeneracy of the primer pool and were also rich in fourfold degenerate residues.

The high overall degeneracy of the primer pools was in part addressed by introducing inosine to the $3^{\text {rd }}$ position 'wobble' base if the position was fully degenerate (ie specified a A T G or C), but not within 3 bp of the 3 ' end of the primer as this is known to adversely affect priming. Inosine is known to base pair with all bases and has been often been used to reduce the degeneracy of a degenerate PCR primer pool (McPherson et al, 1991).

Degenerate PCR using primers derived from partial protein sequence often differs from conventional PCR in that the relative positions of the peptide sequence and hence the degenerate primers position is unknown. Such was the case with NTE and this significantly increased the complexity of the experiments, because two sets of PCR reactions needed to be performed to cover all possible permutations of the primer positions. In addition to the primer pairings, single primer controls are necessary since previous experience (as well as Hiltunen et al, 1994) has shown that degenerate primers can produce artifactual PCR products. Finally, negative controls omitting the cDNA are necessary for both the primer pair and single primer reactions to test for contamination and primer dimer formation.

The problem with the increased number of reactions in an experiment is not the large number of reactions, but the proportionally increased chance of spurious product formation. Without reliable information of how large a genuine 
product should be, there is no simple method of choosing candidates for further investigation. Chapter 5 describes how two of the peptide sequences were putatively positioned relative to each other and used in a degenerate PCR experiment. Unfortunately there was little other positional information aside from the minimum distance that the sequence was from the C-terminal of NTE, provided by the fragment sizing that accompanied each peptide sequence determination (Figure 3.7). One method that could take advantage of this information was Rapid Amplification of cDNA Ends (RACE).

\subsubsection{Rapid Amplification of cDNA Ends}

RACE is a PCR-based method for the amplification and cloning of the $5^{\prime}$ or $3^{\prime}$ ends of a gene (called 5' RACE and 3' RACE respectively), using either DNA or degenerate DNA sequence for product selection (Frohman et al, 1990 and Lee and Caskey, 1990).

In RACE, CDNA is synthesised with a known DNA 'anchor' sequence attached to either the $3^{\prime}$ or $5^{\prime}$ end of the product. Then the desired gene is amplified using a Gene Specific Primer (GSP) derived from the known or inferred gene sequence and an anchor primer (Figure 1.12). The principle advantages of the RACE protocol are that only a single GSP is required for a reaction. In addition, since the peptide sequence and hence the degenerate primers are of known orientation relative to the mRNA, no extra reactions need to be run to test alternate orientations, unless a second round of RACE is performed to nest pairs of degenerate primers. 


\subsubsection{5' RACE}

In 5' RACE (amplification of the 5' end of a CDNA) the CDNA is synthesised from RNA using reverse transcriptase and a poly-dT oligonucleotide primer. The anchor sequence can then be enzymatically attached to the 5' end of the CDNA (Frohman, 1990). This second stage in CDNA synthesis was the principal factor against using the 5' RACE method for cloning NTE, since the additional synthetic stages are poorly reproducible and can lead to truncated CDNAs which may omit the DNA sequence encoding the NTE peptides (Edwards et al, 1995).

The advantage of 5' RACE in the context of cloning NTE is that the more reliable $\mathrm{N}$-terminal peptide sequence is used to design the 3 ' end of the degenerate primers; since the $3^{\prime}$ end is most sensitive to mismatch, it is most important for the sequence in this region to be accurate. The principal disadvantage of 5 ' RACE is that the PCR product makes little use of any additional peptide sequence. In each Edman sequencing run there is normally sequence at the C-terminal which cannot be used to design degenerate primers because its either too heterogeneous or, more often, the Edman sequencing reaction is beginning to fail so the residues returned are unreliable. This sequence though too untrustworthy for primer design can be useful in rapidly identifying putative NTE clones since the sequence adjoining that produced by the degenerate primer should at least resemble the unused sequence.

\subsubsection{3' RACE}

3' RACE was used in the first attempt at cloning NTE using degenerate primers (see Chapter 4). The CDNA used in the 3' RACE protocol requires only a small change to the standard CDNA synthesis protocol; this involves attaching an adapter/anchor primer construct consisting of a poly-dT region (to anneal to the poly-A tail of mRNA) and a unique DNA sequence to 'tag' the 5 ' end of the CDNA and provide a target for a reverse anchor primer (Figure 1.12). One advantage of the 3' RACE method is it allows an estimate of the smallest PCR product that the 
RACE reaction could theoretically produce, based on the size of the peptide fragments the original protein sequence came from (Figure 4.1). However, predicted product sizes only provide a rough guide to product size since 3' RACE is known to be able to produce truncated products if the poly-dT primer anneals to an adenine rich region within the mRNA sequence (Schmidt et al, 1993).

\subsubsection{Other cloning work performed on NTE}

Besides the cloning attempts described in Chapters 4 and 5 , other methods were tried by this research group. The peptide sequences ADLT and DLGL (see Figure 3.7) were used to design three degenerate inosine-containing oligonucelotide probes. These were used to screen 1.6 million colonies from a Clontech pig brain CDNA library at low stringency but no clones were isolated. A second approach utilised the 26 amino acid ADLT (Figure 3.7) peptide sequence. Forward and reverse degenerate primers were designed to residues 1 to 7 and 15 to 21 respectively of the ADLT sequence. A PCR product produced by this primer pair would have produced a 63 bp product which could have been unambiguously identified as ADLT by translation of the 25bp DNA sequence that lay between the degenerate primers. However, despite careful optimisation, the degenerate primers failed to produce any products of the correct size. 
A)<smiles>[R]P([R])([R])=O</smiles>

B)<smiles>[R]P1(=O)OCc2ccccc2O1</smiles>

Figure 1.1 General structure of organophosphorus esters

A) Various substitutions of this general structure are possible, $\mathbf{R}^{1}$ and $\mathbf{R}^{2}$ are usually alkyl or aryl groups bonded either directly to the phosphorous or via an oxygen, sulphur or nitrogen atom. The substituent $X$ may be one of a wide variety of groups; either organic (aliphatic, aromatic or heterocyclic) bound to the phosphate via a labile group (usually oxygen or sulphur), or inorganic (halogen or cyanide groups). The common property of the $X$ group is that it is the most labile of the four bonds attached to phosphorous.

\begin{tabular}{|c|c|c|c|}
\hline Compound & $\mathrm{R} 1$ & $\mathrm{R} 2$ & $x$ \\
\hline Sarin & Me- & Prio- & $-F$ \\
\hline DFP & Prio- & Prio- & $-F$ \\
\hline Paraoxon & Eto- & Eto- & $-\mathrm{OPh}-4-\mathrm{NO}_{2}$ \\
\hline Mipafox & $(\mathrm{Me})_{2} \mathrm{CHNH}-$ & $(\mathrm{Me})_{2} \mathrm{CHNH}-$ & $-F$ \\
\hline EPNO & $\mathrm{Ph}-$ & Eto- & $-\mathrm{OPh}-4-\mathrm{NO}_{2}$ \\
\hline
\end{tabular}

B) The saligenin cyclic phosphoryl OP compounds represent a special case of the OP form, in which the $R^{2}$ and $X$, positions are linked by a heterocyclic moiety. the $\mathrm{CH}_{2} \mathrm{O}-\mathrm{P}$ bond is broken when saligenin cyclic phosphoryl compounds from a covalent complex with the serine at the active site of NTE (see Figure 1.3).

Four saligenin phosphate compounds are mentioned in the text:-

Saigenin cyclic o-tolyl phosphate (TSP), in which the methyl group in the heterocyclic ring is hydroxylated and the leaving group is 2 , methylphenol group (see Figure 1.4).

Octyl saligenin cyclic phosphate in which $\mathrm{R}^{1}$ is octanol.

Phenyl O, O' saligenin phosphate (PSP) in which R1 is phenol

Biotinylated saligenin phosphoramidates (ie S9B; see Figure 1.8). 

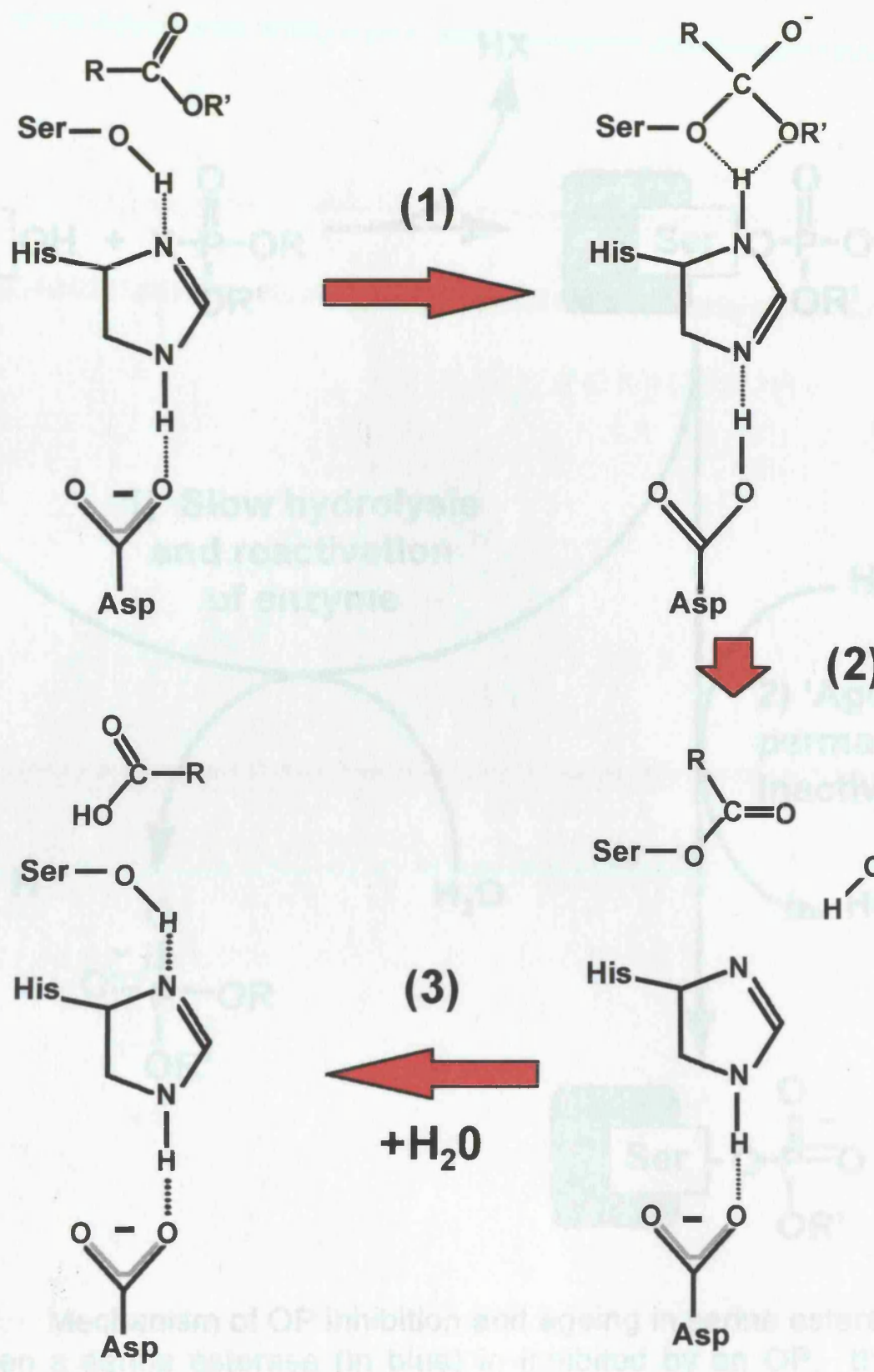<smiles>[R]OC([R])=O</smiles>

(3)
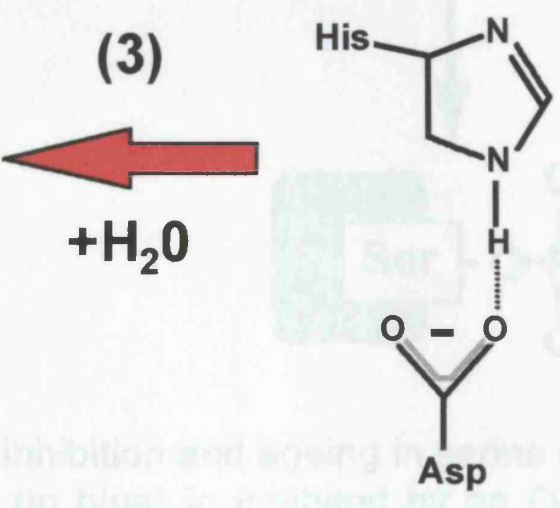

Figure 1.2 Catalytic mechanism of serine esterases.

1) The oxygen of the catalytic serine makes a nucleophilic attack on the carbonyl atom in the ester, to form a tetrahedral Michaelis complex (the negative charge on the oxygen is in stabilised by hydrogen bonding with other portions of the protein; not shown).

2) The Michaelis complex collapses ejecting the labile alcohol group ( $\left.\mathrm{R}^{\prime} \mathrm{OH}\right)$ leaving a bound acyl group $(\mathrm{R}-\mathrm{C}=\mathrm{O})$.

3) The catalytic serine is regenerated by hydrolysis of the acyl group and the carboxylic acid is released from the catalytic cleft (adapted from Simmonds, 1992). 


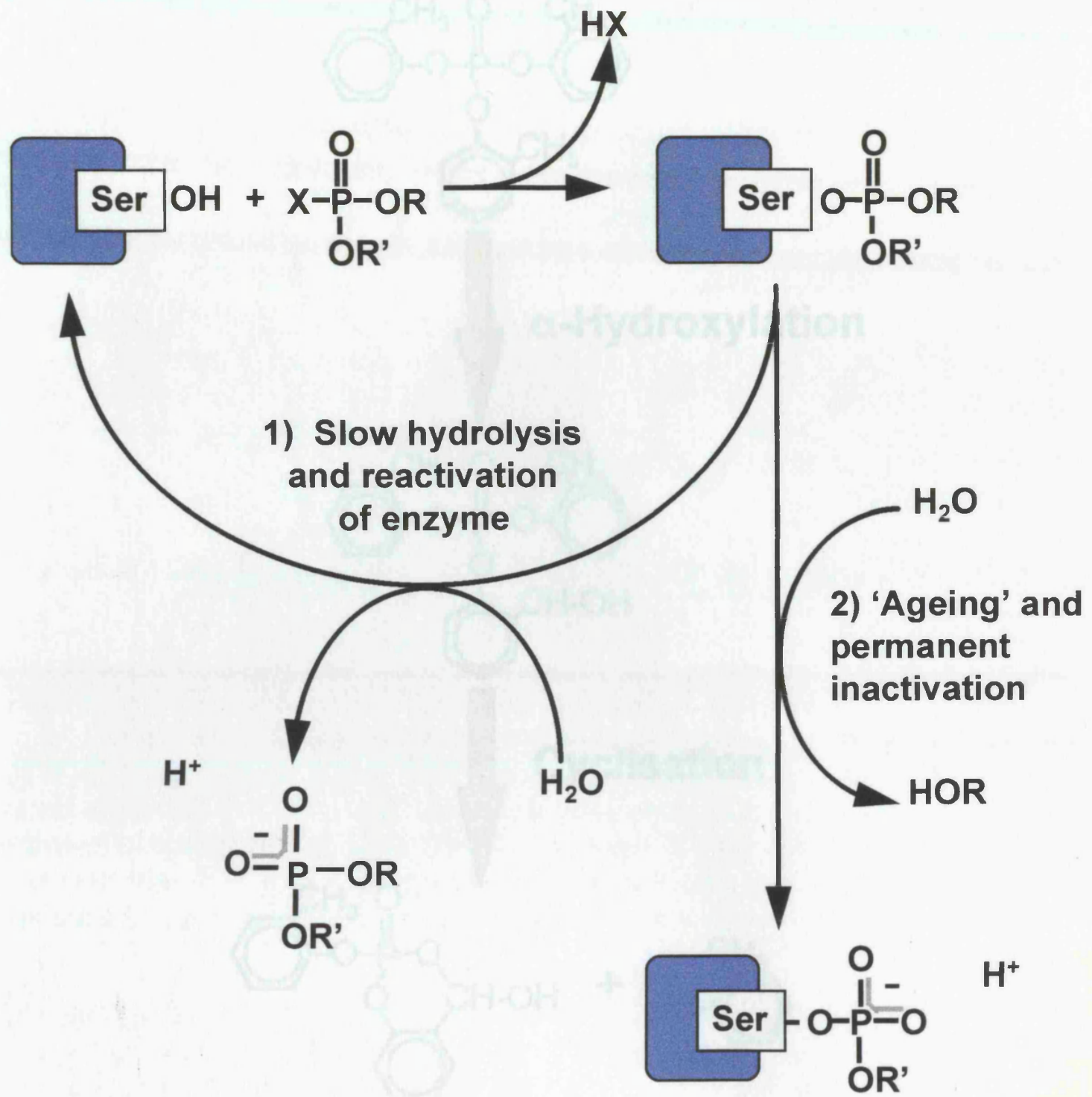

Figure 1.3 Mechanism of OP inhibition and ageing in serine esterases.

When a serine esterase (in blue) in inhibited by an OP, the tetrahedral OP-serine complex is stabilised by the hydrogen bonding that also stabilises the tetrahedral Michaelis complex formed during acylation of the enzyme (see Figure 1.2). The complex is not stable and can under go one of two fates: 1) slow hydrolysis (over a period of hours) removing the OP and regenerating the enzyme, or, 2) 'Ageing' when one of the other phosphate groups are hydrolysed leaving a negative charge on the OP and rendering the inhibition permanent (adapted from Johnson, 1992). 


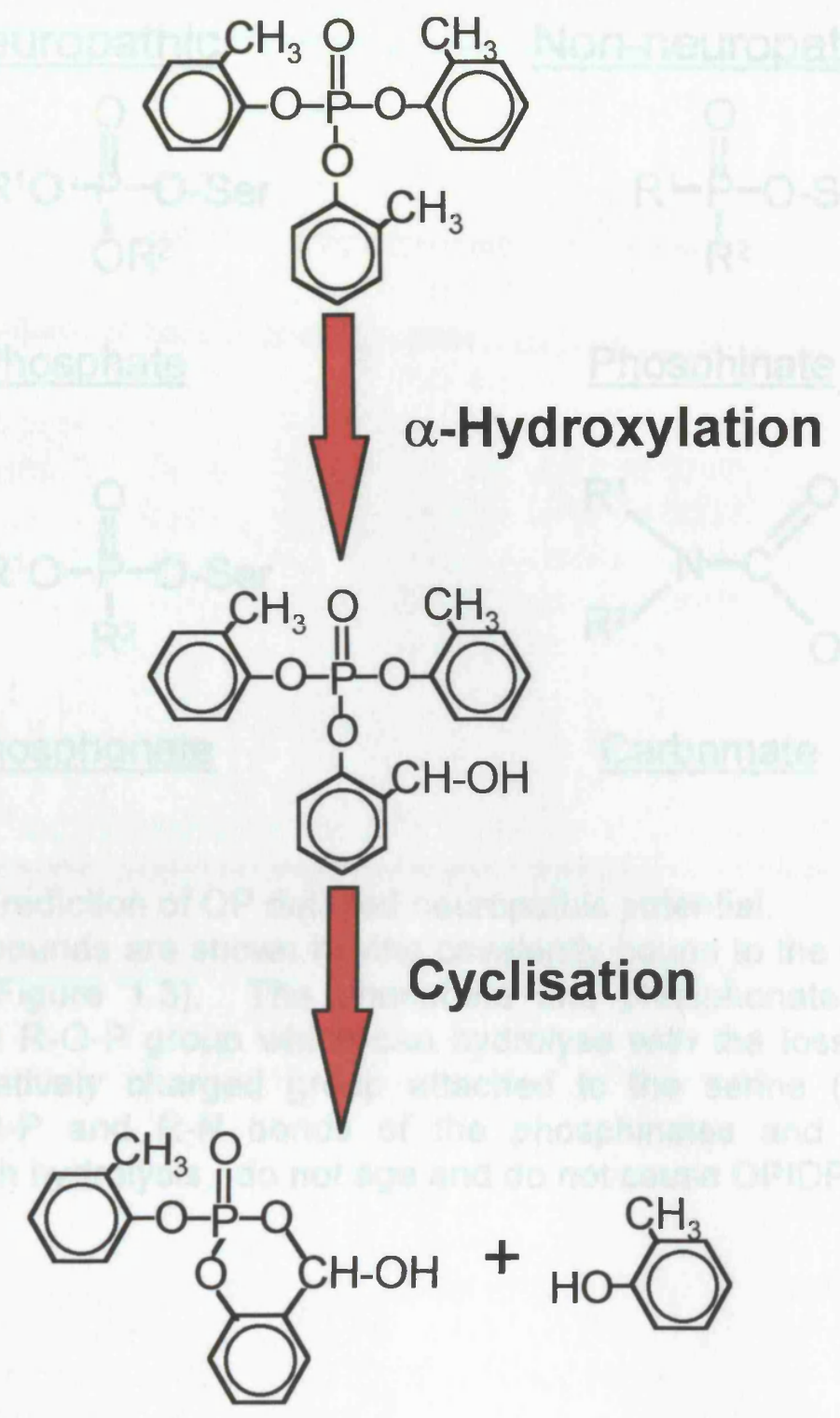

Figure 1.4 Formation of saligenin cyclic o-tolyl phosphate from TOCP. Adapted from Johnson, 1982 


\section{Neuropathic}<smiles>[R20][R]([R20])(=O)O[Ga]</smiles>

Phosphate<smiles>[R20]P([R])(=O)O[Ga]</smiles>

Phosphonate

\section{Non-neuropathic}<smiles>[R]P([R])(=O)O[Ga]</smiles>

Phosphinate<smiles>[R]N([R])C(=O)O[Ga]O</smiles>

Carbamate

Figure 1.5 Prediction of OP delayed neuropathic potential.

All compounds are shown having covalently bound to the active site serine of NTE (see Figure 1.3). The phosphate and phosphonate both contain a relatively labile R-O-P group which can hydrolyse with the loss of the $R$ group leaving a negatively charged group attached to the serine (Figure 1.3). In contrast the R-P and R-N bonds of the phosphinates and carbamates are resistant to such hydrolysis, do not age and do not cause OPIDP. 


\begin{tabular}{llll} 
Tissue & NTE activity & Tissue & NTE activity \\
\cline { 2 - 3 } B brain & & \% brain \\
Brain & 100 & Muscle (pectoralis) & 0 \\
Spinal cord & 23 & Muscle (gastrocnemius) & 0 \\
Dorsal root ganglia & 18 & Liver & 0 \\
Spleen & 60 & Kidney & 0 \\
Spleen lymphocytes & 26 & & \\
Blood lymphocytes & 24 & 10-day embryo brain & 26 \\
Blood T cells & 24 & 14-day embryo brain & 21 \\
Blood B cells & 39 & 17-day embryo brain & 64 \\
Heart & 14 & 20-day embryo brain & 62 \\
Thymus & 69 & 6 week old brain & $100-120$ \\
lleum & 94 & &
\end{tabular}

Figure 1.6 Tissue distribution of NTE activity as a percentage of adult hen brain NTE activity (reproduced from Johnson, 1992). 
Edman cycle Residue

number

1

Lysine

Isoleucine

Valine

Phenylalanine

Alanine

2

Glutamic acid

Valine

Alanine

3

Lysine

Alanine

Glycine

4

Glutamic acid

5

Alanine

Dehydroalanine

6

Methionine

Lysine

7

Glycine

Figure 1.7 Putative N-terminal sequence around the NTE catalytic serine.

An enriched ( $2 \%$ pure) [ $\left.{ }^{3} \mathrm{H}\right] \mathrm{DFP}$-labelled-NTE fraction was first digested with $V 8$ protease and a $16 \mathrm{kDa}\left[{ }^{3} \mathrm{H}\right] \mathrm{DFP}$-labelled fragment was purified by preparative SDS-PAGE. This fragment was then thermolysin digested and a $\left[H^{3}\right]$ DFP-labelled peptide fragment HPLC-purified. Edman degradation of this peptide fragment yielded a seven residue heterogeneous sequence. The dehydroalanine in cycle 5 reflects a modified serine; surrounding it can be discerned the serine esterase consensus sequence Gly-Glu-Ser-XXX-Gly (Glynn et al, 1993). 
<smiles>O=C(CCC1SCC2NC(=O)NC21)NCCNP1(=O)OCc2ccccc2O1</smiles>

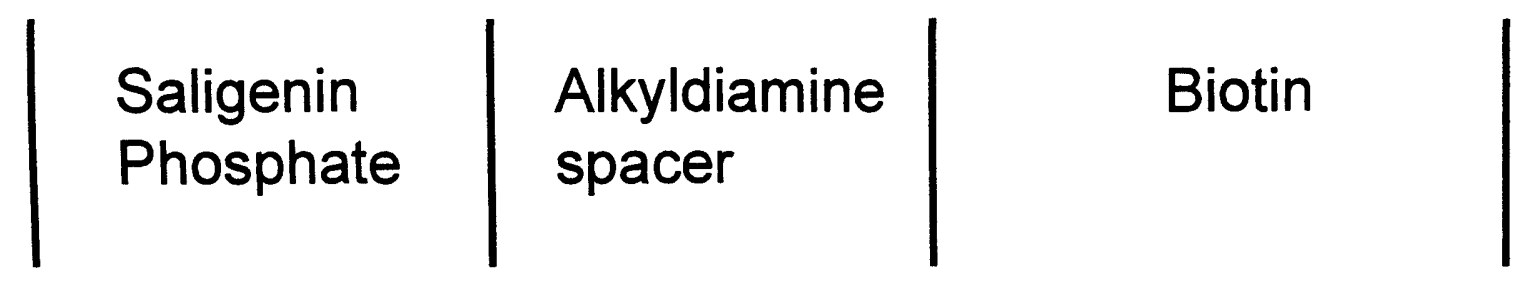

Figure 1.8 General structure of the biotinylated saligenin phosphoramidates used to purify NTE.

A total of six biotinylated saligenin phosphoramidates were synthesised with alkyldiamine spacers of 5 , to 12 methyl carbon atoms in length. These were assayed for inhibition of NTEs phenyl valerate hydrolase activity and a rank order of potency was established to be $n=9,10>8,12>7 \gg 5$. The $n=9$ homologue was selected for subsequent work and designated S9B. Its $\mathrm{I}_{50}$ was determined to be $1.6 \mathrm{nM}$ and it was shown that it could bind to a $155 \mathrm{kDa}$ protein (Glynn et al, 1994). 


\begin{tabular}{|c|c|c|c|c|}
\hline Protein & $\frac{\text { Size }}{(\mathrm{kDa})}$ & Function & $\frac{\text { Tissue }}{\text { location }}$ & Reference \\
\hline $\begin{array}{l}\text { Glutactin } \\
\text { Drosophila }\end{array}$ & 155 & $\begin{array}{l}\text { Cell-cell and cell- } \\
\text { substrate interactions }\end{array}$ & $\begin{array}{l}\text { Neural } \\
\text { Basement } \\
\text { membrane }\end{array}$ & $\begin{array}{l}\text { Olson et al, } \\
(1990)\end{array}$ \\
\hline $\begin{array}{l}\text { Neurotactin } \\
\text { Drosophila }\end{array}$ & 135 & Cell adhesion & Neurons & $\begin{array}{l}\text { Escalera et al, } \\
\text { (1990) }\end{array}$ \\
\hline $\begin{array}{l}\text { Gliotactin } \\
\text { Drosophila }\end{array}$ & 109 & $\begin{array}{l}\text { Formation of blood } \\
\text { nerve barrier }\end{array}$ & Peripheral glia & $\begin{array}{l}\text { Auld et al } \\
\text { (1995) }\end{array}$ \\
\hline $\begin{array}{l}\text { Neuroligin1 } \\
\text { Rat }\end{array}$ & 116 & $\begin{array}{l}\text { Cell-cell and cell- } \\
\text { substrate interactions }\end{array}$ & $\begin{array}{l}\text { Brain neurons } \\
\text { especially } \\
\text { synapses }\end{array}$ & $\begin{array}{l}\text { Ichtchenko et al, } \\
\text { (1995) }\end{array}$ \\
\hline $\begin{array}{l}\text { Neuroligin2 } \\
\text { Rat }\end{array}$ & 94 & As neuroligin1 & as neuroligin1 & $\begin{array}{l}\text { Ichtchenko et al, } \\
\text { (1996) }\end{array}$ \\
\hline $\begin{array}{l}\text { Neuroligin3 } \\
\text { Rat }\end{array}$ & 100 & As neuroligin1 & as neuroligin1 & $\begin{array}{l}\text { Ichtchenko et al, } \\
\text { (1996) }\end{array}$ \\
\hline
\end{tabular}

Figure 1.9 AChE-like enzymes which lack catalytic activity. 


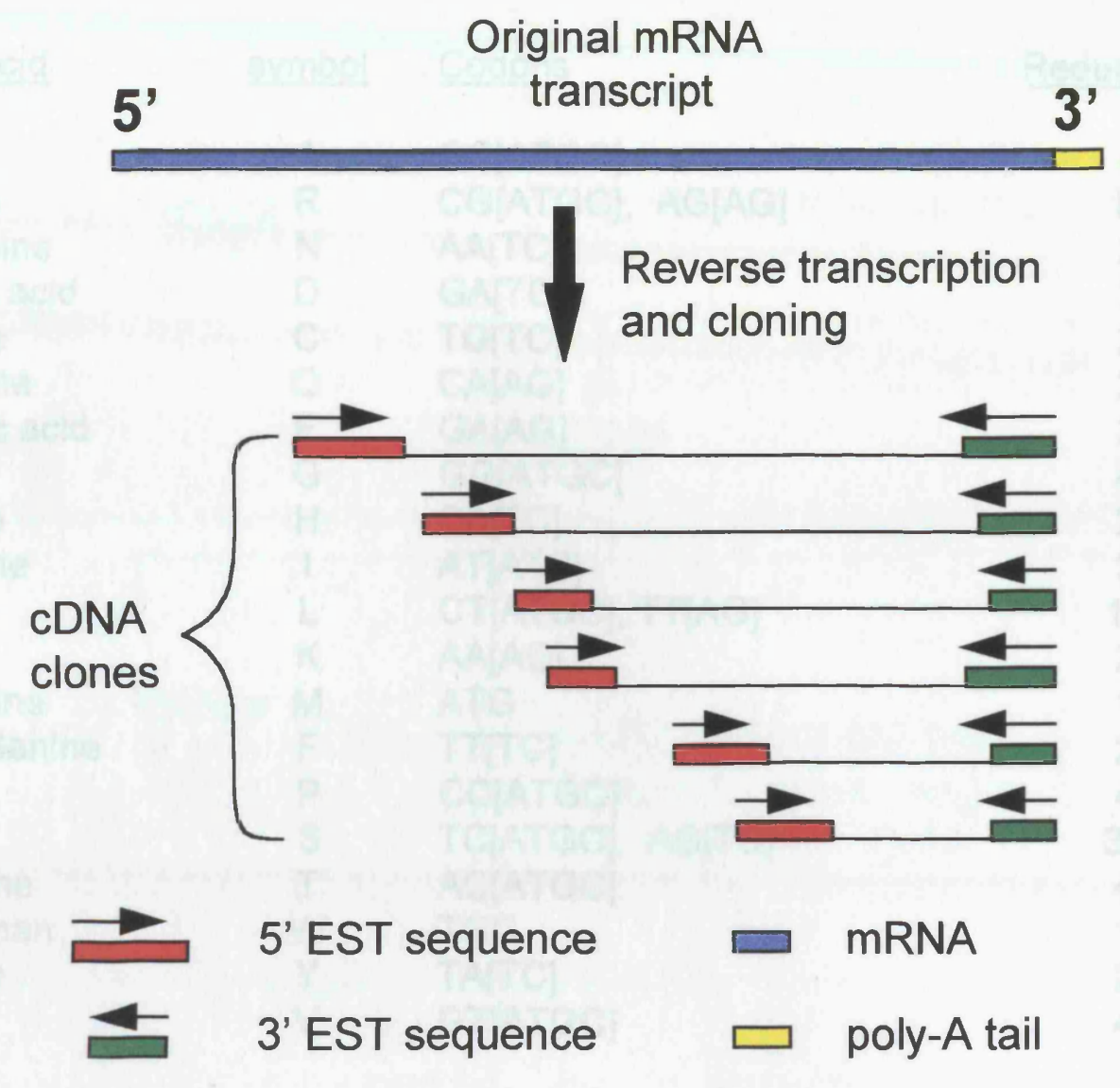

Figure 1.10 Synthesis of cDNA for EST sequencing produces a common 3' end sequence and many 5 ' end sequences from the same gene. 


\section{Amino acid}

Alanine

Arginine

Asparagine

Aspartic acid

Cysteine

Glutamine

Glutamic acid

Glycine

Histidine

Isoleucine

Leucine

Lysine

Methionine

Phenylalanine

Proline

Serine

Threonine

Tryptophan

Tyrosine

Valine symbol Codons

A GC[ATGC]

R CG[ATGC], AG[AG]

N AA[TC]

D GA[TC]

C TG[TC]

Q CA[AG]

E GA[AG]

G GG[ATGC]

H CA[TC]

AT[ATC]

L CTIATGC], TT[AG]

$\mathrm{K}$ AA[AG]

$M \quad$ ATG

F TT[TC]

$P \quad C C[A T G C]$

S TC[ATGC], AG[TC]

$T \quad$ AC[ATGC]

W TGG

$Y \quad T A[T C]$

$V \quad$ GTIATGC]
Redundancy

4

16

2

2

2

2

2

4

2

3

16

2

1

2

4

32

4

1

2

4

Figure 1.11 Amino acid degeneracy codes. 


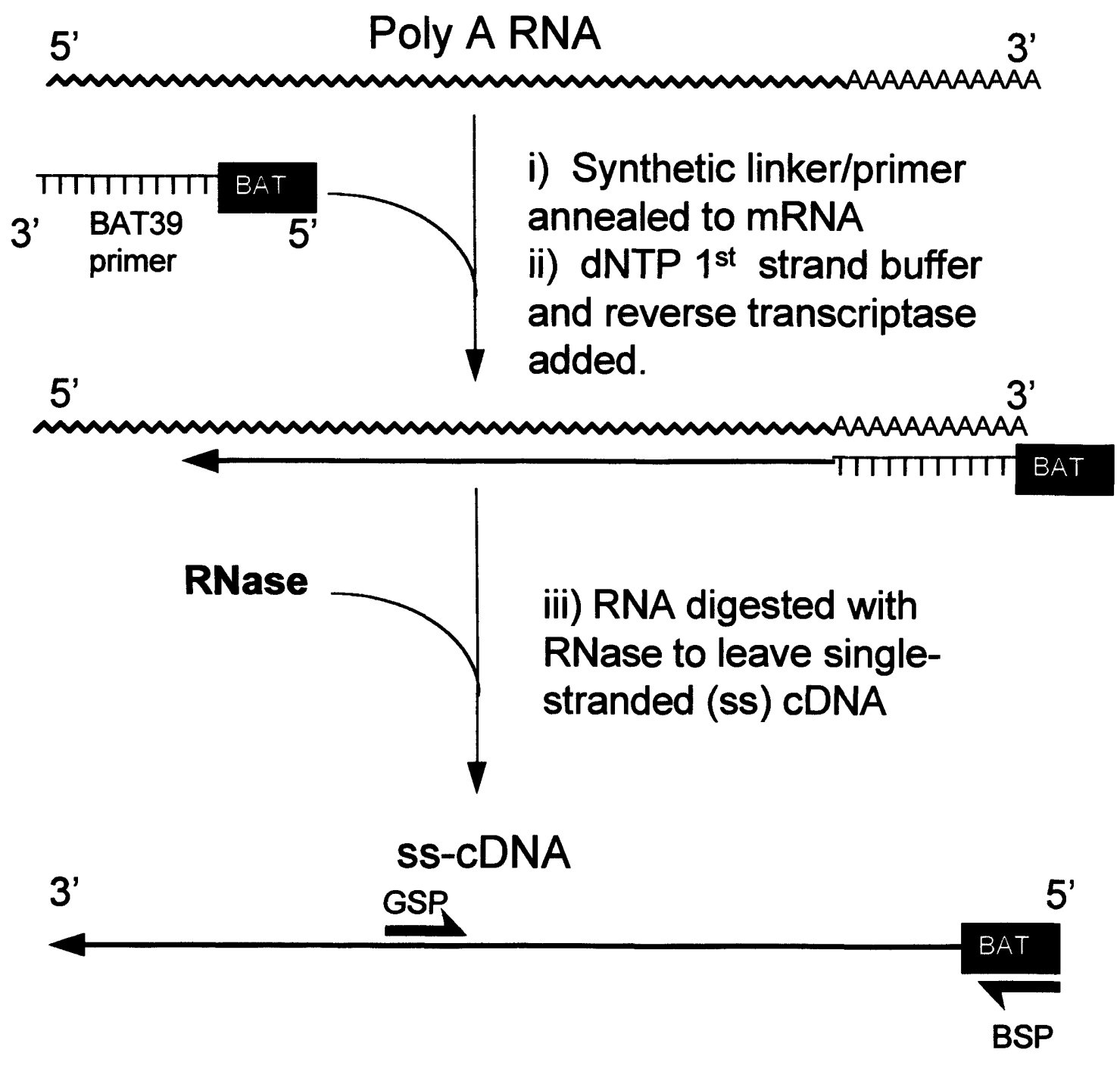

Figure 1.12 Synthesis of CDNA for 3' RACE.

A linker adapter designated BAT39 (see Figure 4.2) consisting of a polydT region at the $3^{\prime}$ end of the primer followed by a unique anchor sequence (BAT39 5' GTTACGGCTGGATGAGTGACTTTTTTTTTTTTTTTTTTT 3') was annealed onto poly-A RNA. Reverse transcriptase was then used to generate the CDNA and the RNA was then removed by digestion with RNase A.

The BAT39-primed CDNA was used in a series of 3' RACE reactions in which a degenerate Gene Specific Primer (GSP) was used in combination with a primer (BSP) made using the BAT anchor sequence (attached to the 5' end of all CDNA templates) as the reverse primer.

In 5' RACE the anchor primer is attached to the 3' end of CDNA (i.e the 5' end of the mRNA). 


\section{Chapter 2 Methods and reagents}

\subsection{Protein methods}

\subsubsection{Preparation of microsomes}

\section{Reagents}

TE buffer

$50 \mathrm{mM}$ TRIS- $\mathrm{HCl}$ pH8.0

$1 \mathrm{mM}$ EDTA

\section{DTT/TE}

TE supplemented with $1 \mathrm{mM}$ dithiothreitol (DTT; Sigma)

$0.9 \% \mathrm{NaCl} / \mathrm{TE}$

$0.9 \%$ Sodium Chloride in TE buffer

\section{Protocol}

A total of $150 \mathrm{~g}$ of frozen brain tissue was thawed, chopped in to 1 litre of DTT/TE and then homogenised in a Waring blender set at low speed. Further DTT/TE was added to a final volume of $1500 \mathrm{ml}$ and distributed evenly in to six $250 \mathrm{ml}$ bottles for a JA-14 rotor (Beckman) and spun at 9000rpm $(12,000 \mathrm{xg})$ at $4^{\circ} \mathrm{C}$ for 15 minutes. The supernatant was decanted and transferred to six $250 \mathrm{ml}$ Type 19 bottles (Beckman), TE was added until the bottles were full whereupon they were spun at $19,000 \mathrm{rpm}(54,000 \mathrm{~g})$ at $4^{\circ} \mathrm{C}$ for 1 hour in a Type 19 Beckman rotor. The supernatant was discarded and the pellets were resuspended in $30 \mathrm{ml}$ $0.9 \% \mathrm{NaCl} / \mathrm{TE}$ using an Ultra-turrax set at medium speed.

The resuspended pellets from three $19 \mathrm{k}$ spins (ie $450 \mathrm{~g}$ of processed brain) were placed in to six type 19 centrifuge bottles and topped up until full with additional $0.9 \% \mathrm{NaCl} / \mathrm{TE}$, then spun at $19,000 \mathrm{rpm}$ at $4^{\circ} \mathrm{C}$ for 1 hour. The supernatant was discarded and each pellet was resuspended in $40 \mathrm{ml} \mathrm{TE}$, dispersing it by Ultra-turrax set at medium speed. A $1 \mathrm{ml}$ aliquot was taken from the resuspended pellets for protein determination (see section 2.1.2) and the 
remaining microsomes were pooled and stored at $-20^{\circ} \mathrm{C}$ in $25 \mathrm{ml}$ aliquots. Under these conditions NTE was stable for approximately two weeks. The typical yield was about $5 \mathrm{mg}$ of microsomal protein per gram of brain tissue processed; at least $3000 \mathrm{mg}$ of microsomal protein was required for the S9B labelling stage (Glynn et al, 1994).

\subsubsection{Protein Determination}

\section{Reagents}

DC protein assay kit (BioRad)

Reagent A an 'alkaline copper tartrate solution' reagent $B$ a 'dilute Folin Reagent'

Reagent $\mathrm{S}$

\section{Protocol}

If the sample contained detergent $20 \mu \mathrm{l}$ of Reagent $\mathrm{S}$ was added per $\mathrm{ml}$ of Reagent A. A standard curve of $0,250,500,750,1000,1500$ and $2000 \mu \mathrm{g} / \mathrm{ml}$ BSA was prepared and $100 \mu$ was pipetted into clean dry test tubes; a $5 x$ dilution of sample was similarly prepared. To each tube $500 \mu$ l of Reagent A was added and vortex mixed, then $4 \mathrm{ml}$ of reagent $B$ was added and the tube was vortexed again. After 15 minutes the absorbance of each reaction was measured at $750 \mathrm{~nm}$. The standards typically produced ODs up to 0.6 (BioRad DC Protein Assay Kit). 


\subsubsection{S9B-labelling and solubilisation of microsomes}

Reagents

TE buffer

50mM TRIS- $\mathrm{HCl} \mathrm{pH} 8.0$

$1 \mathrm{mM}$ EDTA

$0.1 \% \mathrm{DTT} / \mathrm{TE}$

TE supplemented with $0.1 \% \mathrm{DTT}$ (Sigma)

$160 \mu \mathrm{M} \mathrm{S9B}$ in dry dimethylformamide (DMF) Supplied by D. Read (see Glynn et al, 1994)

$10 \%$ SDS solution

\section{Protocol}

Microsomes equivalent to $750 \mathrm{mg}$ of protein were defrosted and diluted with TE to a final concentration of $2 \mathrm{mg} / \mathrm{ml}$ and dispersed by Ultra turrax set at medium speed. The microsomes were then evenly split in to six Type 19 rotor bottles and warmed in a $37^{\circ} \mathrm{C}$ incubator for 15 minutes then $62.5 \mu \mathrm{l}$ of $160 \mu \mathrm{M} \mathrm{S9B}$ in dry DMF was added to give a final concentration 160nM S9B. This was incubated for 20 minutes at $37^{\circ} \mathrm{C}$ after which the bottles were filled to the top with $180 \mathrm{ml} \mathrm{TE}$ and then centrifuged at $19,000 \times \mathrm{xg}$ at $4^{\circ} \mathrm{C}$ for 1 hour. The supernatant was discarded and the pellet resuspended in $0.1 \% \mathrm{DTT} / \mathrm{TE}$ by Ultra-turrax. The resuspended microsomes were pooled in a $500 \mathrm{ml}$ Schott bottle and $5.7 \mathrm{ml}$ of $10 \%$ SDS solution was added to give a final SDS concentration of $0.15 \%$. The bottle was then placed in a boiling waterbath for 7 minutes and subsequently cooled under a under a running tap. This process was repeated a further three times until a total of $3000 \mathrm{mg}$ of microsomal protein had been S9B labelled (Glynn et al, 1994). 
2.1.4 Solubilisation of microsomes and capture of NTE on avidinSepharose

\section{Reagents}

TE buffer

$50 \mathrm{mM}$ TRIS-HCl pH8.0

$1 \mathrm{mM}$ EDTA

$0.1 \% \mathrm{DTT} / \mathrm{TE}$

TE supplemented with $0.1 \%$ DTT

DTT/SDS/TE

TE supplemented with $0.1 \%$ DTT and $0.15 \%$ SDS

$10 \%$ SDS solution

$50 \mathrm{ml}$ Falcon tube

Centriprep100 spin column (Amicon)

Avidin-Sepharose -supplied by P. Glynn (Glynn et al, 1994)

\section{Protocol}

A $10 \mathrm{ml}$ column of avidin-Sepharose was packed in to a $2 \mathrm{~cm}$ diameter chromatography column (Pharmacia) and pre-equilibrated by pumping $50 \mathrm{ml}$ of DTT/SDS/TE through at a rate of $80 \mathrm{ml} /$ hour. The SDS-solubilised S9B-labelled microsome extracts were then combined and were pumped through the column overnight at room temperature (a rate of approximately $80 \mathrm{ml} / \mathrm{hour}$ ). In the morning the remaining SDS extract was pumped through at a rate of $450 \mathrm{ml} / \mathrm{hour}$ and the column was washed by pumping through $200 \mathrm{ml} 0.15 \%$ SDS/TE. The avidin-Sepharose slurry was transfered to a $50 \mathrm{ml}$ Falcon tube and washed a further four times by resuspending in $40 \mathrm{ml} 0.15 \% \mathrm{SDS} / \mathrm{TE}$, then pelleting by centrifugation at $1500 \mathrm{rpm}(400 \mathrm{xg})$ for 2 minutes without braking in a benchtop centrifuge. The bound protein was then eluted by resuspending in $30 \mathrm{ml}$ of $1 \% \mathrm{SDS} / 1 \% \mathrm{DTT}$ in $0.2 \times \mathrm{TE}$ and then immersion in boiling water for 7 minutes. The extract was cooled to room temperature and the avidin-Sepharose removed, pelleting by centrifugation at $1500 \mathrm{rpm}(400 \mathrm{xg})$ for 5 minutes after which the supernatant was decanted. Trace contamination from carried over avidin- 
Sepharose was removed by a secondary spin. The supernatant was then concentrated down to a $2 \mathrm{ml}$ volume using a Centriprep100 spin column (Amicon; three 1600rpm spins were required: 45 minutes, 20 minutes and 10 minutes discarding filtrate between spins), A $50 \mu \mathrm{l}$ aliquot of the concentrate was taken (designated 'avidin eluate') and the rest of the concentrate was submitted to $D$. Read for preparative-PAGE to remove the remaining contaminant proteins (Glynn et al, 1994).

\subsubsection{Analysis of prep-PAGE fractions}

\section{Reagents}

SDS sample buffer

$10 \%$ SDS

$10 \%$ glycerol

$62 \mathrm{mM}$ TRIS- $\mathrm{HCl} \mathrm{pH} 6.8$

$\sim 0.1 \%$ bromophenol blue

$0.25 \%$ Coommassie blue in $10 \%$ acetic acid $/ 50 \%$ methanol

Microcon 100 columns (Amicon)

Bovine Serum Albumin (BSA) concentration standards

$2.5,5,7.5$ or $10 \mu \mathrm{g} / \mathrm{ml} \mathrm{BSA} \mathrm{dissolved} \mathrm{in} 2 \%$ sample buffer and $0.1 \%$ DTT

\section{Protocol}

Fractions 20 to 32 from the prep-PAGE usually contained the $155 \mathrm{kDa}$ NTE protein band. An aliquot $(40 \mu \mathrm{l})$ from each fraction was mixed with $10 \mu \mathrm{l}$ of sample buffer and the mix boiled for 2 minutes, then $30 \mu \mathrm{l}$ was loaded on to a $7.5 \%$ SDSPAGE gel along with $30 \mu$ l the avidin eluate $(40 \mu l$ avidin eluate $+10 \mu l$ sample buffer). The SDS-PAGE gel (Section 2.1.7) was run at 200V until the bromomphenol blue marker had reached the bottom of the gel. The gel cassette was dismantled and the gel stained by immersion in $0.25 \%$ Coommassie blue for 20 minutes and destained as described in the SDS-PAGE protocol (Section 2.1.7). Fractions that contained the $155 \mathrm{kDa}$ NTE but no $120 \mathrm{kDa}$ polypeptide were selected (normally 4 fractions) and concentrated to 200-300 $\mu$ l using a 
Microcon100 spin columns. $500 \mu$ l of sample was spun in each column for 15 minutes at $3000 \mathrm{rpm}(720 \mathrm{xg})$ in a benchtop microcentrifuge, the flowthrough was discarded and the retentates combined in a single column which was spun at 3000 rpm for a further 10 minutes to give a final sample volume of $200-300 \mu l$.

The pure NTE solution was precipitated by adding 1.1 volumes of acetone and incubating overnight at $-20^{\circ} \mathrm{C}$, reserving $10 \mu \mathrm{l}$ for later analysis. The precipitate was pelleted by spinning at $14,000 \mathrm{rpm}(15,800 \mathrm{xg})$ for 30 minutes in a microcentrifuge and the pellet dried by vacuum centrifuge for 20 minutes. The yield and purity of the pure NTE protein was assessed by running a $3 \mu$ laliquot of the microcon concentrate (diluted to $30 \mu$ with $2 \%$ sample buffer $+0.1 \% \mathrm{DTT}$ ) on a 7.5\% SDS-PAGE gel (Section 2.1.7 with 30 $\mu$ l concentration standard of 150, 300,450 and 600ng BSA (Glynn et al, 1994)

\subsubsection{V8 protease digest}

\section{Reagents}

V8 protease (Sigma)

Novex $4-20 \%$ gradient gel ( $R$ and $D$ systems)

\section{Protocol}

The precipitated NTE was redissolved in $40 \mu \mathrm{l}$ of $0.1 \% \mathrm{SDS} / 1 \%$ DTT in TE buffer and boiled for 3 minutes. A pilot digest of $4 \mu \mathrm{l} \mathrm{NTE}+1 \mu \mathrm{l}$ V8 protease solution was initially run with V8:NTE ratios of $1: 6$ and $1: 18$ (by mass) paired with a blank which contained only the V8 protease. The digests were incubated for 2 hours at $37^{\circ} \mathrm{C}$ and stopped by the addition of $15 \mu \mathrm{l} \%$ sample buffer. The digests were then run on a Novex $4-20 \%$ gradient gel (150V for 1.5 hours) which was stained as per the SDS-PAGE protocol (Section 2.1.7). Once the appropriate ratio of NTE:V8 was determined the remaining NTE was digested under the same conditions then submitted to the Protein and Nucleic Acid (PNAC) sequencing laboratory for peptide sequencing by Edman degradation(Glynn et al, 1994). 


\subsubsection{SDS-Polyacrylamide Gel Electrophoresis}

\section{Reagents}

Solution A (BioRad)

$30 \%$ Acrylamide

$0.8 \%$ Bis-acrylamide

Solution B

1.5M TRIS- $\mathrm{HCl} \mathrm{pH8.8}$

Solution C

$10 \%$ SDS

Solution D

$0.5 \mathrm{MTRIS}-\mathrm{HCl} \mathrm{pH} 6.8$

$10 \%$ Ammonium persulphate (APS; must be made fresh)

SDS sample buffer

$10 \%$ SDS

$10 \%$ glycerol or sucrose

62mM TRIS-HCl pH6.8

$\sim 0.1 \%$ bromophenol blue

SDS-PAGE running buffer ( $x 5$ stock solution per litre)

$15 \mathrm{~g}$ TRIS (base)

$72 \mathrm{~g}$ glycine

$5 \%$ SDS

TEMED (Sigma)

$50 \mathrm{ml}$ syringe

Novex molecular weight size standard ( $R$ and D Systems)

\section{Protocol}

The gel plates were cleaned with methanol and assembled in the gel casting stand. To make $80 \mathrm{ml}$ of $7.5 \%$ acrylamide gel, $39 \mathrm{ml}$ distilled water, $20 \mathrm{ml}$ Solution A, $20 \mathrm{ml}$ of Solution $B$ and $0.8 \mathrm{ml}$ of Solution $C$ were mixed in a Buchner flask and degassed by sealing the flask with a rubber stopper and reducing the pressure for 10 minutes. The polymerisation reaction was initiated by adding 
$160 \mu \mathrm{l} 10 \%$ APS and $60 \mu \mathrm{l}$ TEMED, then the mixture was transferred to the gel cassette filling it to within $20 \mathrm{~mm}$ of the upper edge of the smaller plate. The gel was then overlayed with butan-2-ol to exclude air and accelerate polymerisation.

After a maximum of 1 hour the butan-2-ol was poured off and the top of the gel was washed with several rinses of distilled water. The water was drained from cassette and a comb inserted in to the cassettes. Stacking gel was prepared by mixing $8.5 \mathrm{ml}$ Solution $A, 12.5 \mathrm{ml}$ Solution $B$ and $0.5 \mathrm{ml}$ Solution $D$ with $28 \mathrm{ml}$ of distilled water in a clean Buchner flask. This mixture was degassed for 10 minutes and $500 \mu \mathrm{l} 10 \%$ APS and $50 \mu$ IEMED was added to the mix, which was then transfered to the gel cassette filling to the top of the smaller plate and taking care to ensure no bubbles were trapped by the comb. The stacking gel normally set within 1 hour and gels could be stored at $4^{\circ} \mathrm{C}$ for up to 10 days.

To run the gel, the comb was removed and the gel cassette fitted into a gel tank; this was filled with $1 \times$ SDS running buffer and samples applied to wells in $2 \%$ sample buffer. Novex molecular weight size standards were used throughout the project (Laemmli, 1971). 


\subsection{DNA purification and cloning}

Unless otherwise stated the following techniques were based on ones presented in Sambrook et al, 1989a.

\subsubsection{Agarose gel electrophoresis}

\section{Reagents}

$5 \times T B E$

450mM TRIS-Borate pH8.0

10mM EDTA

Agarose electrophoresis grade (Sigma)

$10 \mathrm{mg} / \mathrm{ml}$ Ethidium bromide (Sigma)

$1 \mathrm{~kb}$ ladder (Gibco)

Loading buffer

$50 \%$ glycerol

Trace ammounts of Bromophenol blue and Xylene cynol

(sufficent to allow visualisation of sample when loading gel)

\section{Protocol}

A $1 \%$ agarose gel in $0.5 \times T B E$ stained with $0.5 \mu \mathrm{g} / \mathrm{ml}$ ethidium bromide was used to resolve DNA fragments throughout the project. $1 \%$ agarose was boiled in $0.5 x$ TBE until the powder was completely dissolved. This was then allowed to cool to less than $45^{\circ} \mathrm{C}$ at which point ethidium bromide was added to a final concentration of $0.5 \mu \mathrm{g} / \mathrm{ml}$. The gel casting tray was then filled to a depth of 5$6 \mathrm{~mm}$ and the well comb was then inserted. After the agarose gel had set the comb was removed the gel loaded into the electrophoresis tank and immersed in $0.5 \times T B E+0.5 \mu \mathrm{g} / \mathrm{ml}$ ethidium bromide. The DNA sample was mixed with 0.1 volumes of loading buffer and pipetted into the wells. The gel was run at 2.5-5 $\mathrm{V} / \mathrm{cm}$, with the anode at the bottom the gel until the DNA bands of interest were satisfactorily resolved (monitoring by occasional viewing under UV light). Gels were photographed with Polaroid Type 53 film. 
The Gibco 1kb ladder was used throughout the project for sizeing DNA fragments. The ladder consists of bands of $75,134,154,201,220,298,344$, $396,506,517,1018,1636,2036,3054,4072,5090,6108,7126,8144$, $9162,10180,11198$ and $12216 \mathrm{bp}$ in size. Figure 5.3 provides a good example of the first 12 bands, the bottom most being the $75 \mathrm{bp}$ in size.

\subsubsection{PCR product purification by electroelution.}

\section{Reagents}

Dialysis tubing

$2 \%$ sodium bicarbonate

5 XTBE

450mM TRIS-Borate pH8.0

$10 \mathrm{mM}$ EDTA

TE buffer

$10 \mathrm{mM}$ TRIS- $\mathrm{HCl} \mathrm{pH} 8.0$

$1 \mathrm{mM}$ EDTA

\section{Protocol}

The desired PCR product band was resolved by agarose gel electrophoresis and visualised with ethidium bromide under ultraviolet (UV) light (Section 2.2.1). A clean scalpel was used to cut the band from the gel (keeping the exposure to UV light to an minimum to reduce DNA crosslinking) and the agarose gel plug was placed along with $2-3 \mathrm{ml} 0.5 \times \mathrm{TBE}$ in a short section of dialysis tubing (which was prepared by boiling for 10 minutes in a $2 \%$ solution of sodium bicarbonate, followed by thorough washing in distilled water). The dialysis tubing was sealed at each end by double knotting the tube and placed perpendicular to the current in an electrophoresis tank filled with 0.5xTBE and a voltage of $5 \mathrm{~V} / \mathrm{cm}$ placed across the tank for 2 hours. The DNA accumulated on the positively charged (anode) side of the dialysis tubing and was visualised under UV light. DNA bound to the dialysis bag was then dislodged by reversing the polarity for 1 minute then decanting the contents of the dialysis bag 
(excluding the agarose gel plug). The DNA was recovered by phenol/chloroform extraction and ethanol precipitation (Section 2.2.4) and redissolved in $10 \mathrm{mM}$ TRIS-HCl pH8.0 or TE.

\subsubsection{PCR Product purification by QIAspin kit}

\section{Reagents}

Buffer PB (QIAGEN Proprietary buffer)

Buffer PE (QIAGEN Proprietary buffer)

Elution buffer

$10 \mathrm{mM}$ TRIS-HCl pH8.0

QIAquick spin column (QIAGEN)

\section{Protocol}

Five volumes of buffer PB were added per 1 volume of PCR reaction, and the mixture was applied to a QIAquick spin column. After spinning at $\geq 10,000 \times \mathrm{xg}$ for 30-60 seconds in a $2 \mathrm{ml}$ collection tube, the flowthrough was discarded and $0.75 \mathrm{ml}$ buffer PE was applied to the column which was then spun as before, the flowthrough was again discarded. The empty spin column was spun briefly to remove residual buffer PE. Elution buffer $(50 \mu l)$ was then applied to the spin column which was placed in a sterile Eppendorf tube and elution buffer containing the DNA was spun out at $\geq 10,000 \times$ (QIAGEN, 1997). 


\subsubsection{Phenol extraction and ethanol precipitation}

\section{Reagents}

Phenol/chloroform

1 volume equilibrated phenol (Fisons)

1 volume chloroform (Fisons)

TE buffer

$10 \mathrm{mM}$ TRIS- $\mathrm{HCl} \mathrm{pH} 8.0$

$1 \mathrm{mM}$ EDTA

3M Sodium Acetate $\mathrm{pH} 5.2$

Absolute ethanol

$70 \%$ ethanol

\section{Protocol}

An equal volume of phenol/chloroform was added to the solution to be treated. The mixture was vortexed until the phenol/chloroform became emulsified and then spin at $\geq 10,000 \times \mathrm{g}$ for 2 minutes until the phases separated. The upper aqueous phase was decanted and the procedure repeated prior to ethanol precipitation.

$3 \mathrm{M}$ sodium acetate ( 0.1 volume) and 2 volumes of ethanol were mixed with the sample, and then incubated at $-20^{\circ} \mathrm{C}$ for 1 hour. The sample was centrifuged at $>10,000 \times \mathrm{xg}$ for 30 minutes then the supernatant was decanted and the pellet rinsed in $500 \mu \mathrm{l} 70 \%$ ethanol. After spinning for 5 minutes and removal of the ethanol the pellet was allowed to air dry prior to redissolving in TE or water. 


\subsubsection{Ethanol precipitation of oligonucelotides}

\section{Reagents}

Absolute ethanol

$3 \mathrm{M}$ Sodium acetate $\mathrm{pH} 5.2$

$70 \%$ ethanol solution

$10 \mathrm{mM}$ TRIS-HCl pH 8.0

\section{Protocol}

All oligonucelotides were synthesised by the Protein and Nucleic Acids (PNAC) laboratories and were precipitated by the addition of 0.1 volume $3 \mathrm{M}$ Sodium acetate, 2 volumes of absolute ethanol with 1 volume of primer stock solution and incubated for 1 hour at $-20^{\circ} \mathrm{C}$. After spinning at $>10,000 \times \mathrm{xg}$ for 20 minutes the supernatant was decanted and the pellet washed with $70 \%$ ethanol. The pellet was then vacuum centrifuged until dry and redissolved in $50-200 \mu l$ $10 \mathrm{mM}$ TRIS- $\mathrm{HCl} \mathrm{pH} 8.0$. Primer concentration was determined by $O D^{260}$ absorbance $\left(O D^{260} \quad 1=20 \mu \mathrm{g} / \mathrm{ml} \approx 3 \mu \mathrm{M}\right)$.

\subsubsection{Purification of high molecular weight genomic DNA.}

\section{Reagents}

Liquid Nitrogen $\left(\mathrm{LN}_{2}\right)$

Extraction buffer

$10 \mathrm{mM}$ TRIS- $\mathrm{HCl} \mathrm{pH} 8.0$

100mM EDTA

20 $\mu \mathrm{g} / \mathrm{ml}$ Pancreatic RNase A (Gibco)

$0.5 \%$ SDS

Proteinase K (Gibco)

Equilibrated Phenol (Fisons)

$10 \mathrm{M}$ ammonium acetate

Absolute ethanol

$70 \%$ ethanol 


\section{TE buffer}

10mM TRIS- $\mathrm{HCl} \mathrm{pH} 8.0$

$1 \mathrm{mM}$ EDTA

General notes: To prevent premature melting of the pulverised brain powder any item which came into contact with the powder was exhaustively pre-chilled in $\mathrm{LN}_{2}$ until almost no bubbles rose from the object.

\section{Protocol}

Approximately $20-40 \mathrm{~g}$ of frozen $\left(-20^{\circ} \mathrm{C}\right)$ pig brain was allowed to warm sufficiently to permit the tissue to be cut with difficulty, but it not allowed to fully defrost. The tissue was cut in to chunks of $\sim 1-3 \mathrm{~cm}^{3}$ volume and immediately dropped into $\mathrm{LN}_{2}$ and allowed to freeze completely (5-10 minutes). Meanwhile the stainless steel waring blender was carefully dried, filled $1 / 3^{\text {rd }}$ full with $L_{2}$ and the temperature allowed to equilibrate. The $\mathrm{LN}_{2}$ and brain chunks were then poured in to the blender and $\mathrm{LN}_{2}$ was added/removed to ensure the blades were covered to a depth of 1-2 inches (but no more as the nitrogen tends to splash out when the blender ran).

The lid was fitted to the blender and firmly held down (wearing insulating gloves and safety specs) then the blender was placed behind a screen and then run in short bursts $(\sim 1$ second) holding the lid down and allowing time for gas pressure to release between runs. Homogenisation was continued until the until the grinding sound becomes more even, checking regularly to ensure the blades stayed well covered with $\mathrm{LN}_{2}$ and topping it up when necessary.

Once the tissue was sufficiently pulverised (ie reduced to a powder with no large $>2 \mathrm{~mm}$ chunks) the $\mathrm{LN}_{2}$ was transferred along with the powdered brain to a pre-chilled plastic beaker and the $\mathrm{LN}_{2}$ was carefully allowed to boil off to leave a pink 'cake' (still moist with $\mathrm{LN}_{2}$ ) which was transferred in to (pre-chilled) $50 \mathrm{ml}$ Falcon tubes. These could be stored almost indefinitely at $-70^{\circ} \mathrm{C}$, however the lids were punctured (with a 18 gauge needle) to allow residual $\mathrm{LN}_{2}$ to boil off and 
escape (the lids may be replaced after 2 weeks although samples have been successfully stored without this precaution for a year without obvious ill effect).

Seven grams of powdered brain was incrementally added to $70 \mathrm{ml}$ extraction buffer in a $250 \mathrm{ml}$ Schott bottle, stirring after each addition of powder to prevent the powder freezing into aggregates. The extraction mix was incubated for 1 hour at $37^{\circ} \mathrm{C}$, swirling occasionally, then $7 \mathrm{mg}$ of proteinase $\mathrm{K}$ (for a final concentration of $100 \mu \mathrm{g} / \mathrm{ml}$ ) was added and incubated at $50^{\circ} \mathrm{C}$ overnight, swirling periodically for the first few hours.

The proteinase $K$ digest was cooled to room temperature and 1 volume of equilibrated phenol added. This was gently mixed by hand, turning the bottle end over end until the contents were thoroughly emulsified. The phenol emulsion was transferred to $50 \mathrm{ml}$ Falcon tubes and centrifuged at $3000 \mathrm{xg}$ for 45 minutes. The aqueous supernatant was gently decanted in to a clean $250 \mathrm{ml}$ Schott bottle. The phenol extraction was repeated a further two times prior to ethanol precipitation.

A total of 0.2 volumes for $10 \mathrm{M}$ ammonium acetate and 2 volumes of ethanol was added to the aqueous phase and swirled gently to mix. The floating precipitated DNA was fished with a bent glass rod and transfered in to $20 \mathrm{ml}$ of $70 \%$ ethanol. Then the precipitate was spun down at $3000 \mathrm{xg}$ for 5 to 10 minutes, the supernatant discarded and a further $70 \%$ ethanol solution added and the precipitate spun down again. As much ethanol as possible was removed and the pellet allowed to air dry (without allowing the DNA to dry out completely).

The pellet was covered with $3.5 \mathrm{ml}$ of TE and the DNA redissolved by gently rocking at room temperature for at least 24 hours. The DNA concentration was determined by absorbance at $O D^{260}\left(O D^{260} 1=50 \mu \mathrm{g} / \mathrm{ml}\right)$ and protein contamination assessed by measuring $O D^{260} / O D^{280}$ and ensuring ratio was $>1.8$. The typical yield of DNA was approximately $1 \mathrm{mg}$ DNA per gram of tissue. 


\subsubsection{DNA ligation with compatible sticky ends}

\section{Reagents}

$5 x$ ligation buffer (Gibco)

$10 \mathrm{mM}$ ATP

T4 DNA ligase (Gibco)

20u/ $\mu$ l Calf intestinal alkaline phosphatase (CIAP; Gibco)

10x Dephosphorylation buffer

$500 \mathrm{mM}$ TRIS-HCl pH 8.5

$10 \mathrm{mM}$ EDTA

\section{Protocol}

pBluescript (Stratagene) was the standard vector for cloning and subcloning. After a double digestion the vector was gel-purified and dephosphorylated by incubation with 1 unit of CIAP in 1x Dephosphorylation buffer. The CIAP was subsequently heat inactivated at $65^{\circ} \mathrm{C}$ for 15 minutes. Of the CIAP-treated vector 50ng was incubated in a 3:1 insert:vector molar ratio with $1 \mu$ ligation buffer, $1 \mu \mathrm{l}$ ATP, $1 \mathrm{u}$ T4 DNA ligase in a $10 \mu \mathrm{l}$ reaction volume for either 3 hours at $15^{\circ} \mathrm{C}$ or overnight at $4^{\circ} \mathrm{C}$ subsequently $5 \mu$ of the ligation mix was used to transform supercompetent XL1-Blue cells (see Section 2.2.10).

\subsection{8 pGEM-T cloning of PCR products}

\section{Reagents}

10xT4 ligase buffer (Promega)

pGEM-T vector $(50 \mathrm{ng} / \mu \mathrm{l})$

T4 DNA ligase

distilled water

\section{Protocol}

PCR product was added to 50ng of PGEM-T vector in a molar ratio of $3: 1$, in a control reaction $2 \mu \mathrm{l}$ of control insert (supplied with kit) was added to 50ng 
PGEM-T vector and a negative control was included which only contained PGEM$T$ vector. To each of the three reactions $1 \mu$ l of T4 ligase buffer and distilled water was added for a final volume of $9 \mu \mathrm{l}$. T4 $(1 \mu \mathrm{l})$ ligase was then added and the mixture was incubated at $15^{\circ} \mathrm{C}$ for three hours. An aliquot $(5 \mu \mathrm{l})$ was withdrawn for transformation in to high efficiency competent cells (see Section 2.2.10) and the remainder stored at $4^{\circ} \mathrm{C}$ (Promega, 1996).

\subsection{9 pCRscript cloning of PCR products}

\section{Reagents}

$10 \mathrm{mM}$ dNTP mix ( $2.5 \mathrm{mM}$ of each nucleotide)

0.5 units/ $\mu$ l Pfu polymerase

$10 x$ polishing buffer

$100 \mathrm{mM}$ Potassium chloride

$100 \mathrm{mM}$ Ammonium sulphate

$200 \mathrm{mM}$ TRIS- $\mathrm{HCl} \mathrm{pH} 8.75$

$20 \mathrm{mM}$ Magnesium sulphate

$1 \%$ Triton X-100

$1 \mathrm{mg} / \mathrm{mlBSA}$

pCRscript vector $(10 \mathrm{ng} / \mu \mathrm{l})$

10x pCRscript reaction buffer

$500 \mathrm{mM}$ TRIS-HCI pH 7.5

$70 \mathrm{mM}$ Magnesium chloride

$10 \mathrm{mM}$ DTT

$10 \mathrm{mM}$ ATP

Srfl restriction enzyme $(5 u / \mu l)$

\section{Protocol}

PCR products produced by non-proofreading polymerases were 'polished' to produce blunt ended products for pCRscript cloning by adding $1 \mu \mathrm{l} 10 \mathrm{mM}$ dNTP mix $1.3 \mu \mathrm{l} 10 \mathrm{x}$ polishing buffer and $1 \mu \mathrm{l}$ Pfu polymerase to $10 \mu \mathrm{l}$ purified PCR product, overlaying it with $20 \mu \mathrm{l}$ of mineral oil and incubating at $72^{\circ} \mathrm{C}$ for 30 minutes. 
Once cool, $5.5 \mu \mathrm{l}$ of polished PCR products were mixed with $1 \mu \mathrm{l} \mathrm{pCRscript}$ vector, $1 \mu \mathrm{l}$ pCRscript $10 \mathrm{x}$ reaction buffer, $1 \mu \mathrm{l}$ ATP and $1 \mu \mathrm{l}$ Srfl restriction enzyme. The mix was incubated at room temperature for 1 hour then the reaction was stopped by heating to $65^{\circ} \mathrm{C}$ for 10 minutes; $5 \mu l$ of the reaction mix was then withdrawn for transformation in to supercompetent cells (see Section 2.2.10). A positive control using a supplied control insert and a negative control in which only pCRscript was added to the reaction mix were also included in each ligation experiment (Stratagene, 1997).

\subsubsection{Transformation of ligated products}

\section{Reagents}

NZY/Glucose broth (per litre)

$5 \mathrm{~g}$ Sodium chloride

$2 \mathrm{~g}$ Magnesium sulphate (hydrated)

$5 \mathrm{~g}$ bacto-yeast extract

$10 \mathrm{~g}$ casein hydrolysate

$\mathrm{pH}$ was adjusted to 7.5 with $\mathrm{NaOH}$ and then the solution was autoclaved and allowed to cool prior the addition of $20 \mathrm{ml}$ sterile $20 \%$ glucose solution.

Epicurian Coli XL1-Blue MRF' Kan supercompetent cells (Stratagene)

JM109 High efficiency competent cells (Promega)

$15 \mathrm{ml}$ Falcon tubes

L-agar $/ 50 \mu \mathrm{g} / \mathrm{ml}$ ampicillin (per liter)

$10 \mathrm{~g}$ bacto-typtone

$5 \mathrm{~g}$ bacto-yeast extract

$10 \mathrm{~g}$ Sodium Chloride

$15 \mathrm{~g}$ bacto-agar

The $\mathrm{pH}$ was adjusted to 7.0 with $\mathrm{NaOH}$ and autoclaved; when cool $\left(\sim 45^{\circ} \mathrm{C}\right)$ ampicillin was added to a final concentration of $50 \mu \mathrm{g} / \mathrm{ml}$.

$0.2 \mathrm{M}$ IPTG (Isopropyl-1-thio- $\beta$-D-galactopyranoside) in distilled water

$10 \%$

$X$-gal

(5-bromo-4-chloro-3-indoyl- $\beta$-galactopyranoside)

in

dimethylformamide 


\section{Protocol}

The JM109 cells were used to clone the PGEM-T vector, XL1-Blue were used for all transformations involving pBluescript and pCRscript.

The supercompetent cells were stored at $-70^{\circ} \mathrm{C}$, thawed on ice and $3.5 \mu \mathrm{l}$ of $1.44 \mathrm{M} \beta$-mercaptoethanol was added to $200 \mu \mathrm{l}$ of the XL1-Blue cells. $50 \mu \mathrm{l}$ of competent cells were gently dispensed into four pre-chilled $15 \mathrm{ml}$ Falcon tubes and $5 \mu \mathrm{l}$ of the ligation mixes added (one sample tube, one positive control and one negative control; $0.1 \mathrm{ng}$ of control plasmid (pUC18) was added to the remaining tube). All four tubes were incubated on ice for 10 minutes, then placed in a $42^{\circ} \mathrm{C}$ water bath for 45 seconds and quickly returned to the ice to cool for 2 minutes. $450 \mu$ l of NZY/glucose was then added and the transformation mix and then placed in a shaking (225-250rpm) incubater at $37^{\circ} \mathrm{C}$ for 1 hour.

The L-agar ampicillin plates were poured in $150 \mathrm{~mm}$ petri dishes and allowed to set, then $60 \mu \mathrm{l}$ of $0.2 \mathrm{M} \mathrm{IPTG}$ and $60 \mu \mathrm{l}$ of $10 \% \mathrm{X}$-gal was spread over the surface of the agar at least 30 minutes prior to the addition of the transformed cells. All of the sample transformation was spread on the L-agar/amp/Xgal/IPTG plates $(\sim 100 \mu \mathrm{l}$ per $15 \mathrm{~cm}$ petri dish) and $50 \mu \mathrm{l}$ of the three control reactions were plated out and grown overnight at $37^{\circ} \mathrm{C}$ (Stratagene, 1997). Recombinant clones were selected by blue/white selection or hybridisation screening (section 2.2.4). 


\subsection{PCR Methods}

These methods were based on McPherson et al 1993 and 1995.

\subsubsection{General PCR and RACE methods}

Due to the variable nature of the PCR protocol, several different cycling conditions and buffers were used. Rather than list the exact parameters for each experiment here, they are listed with the pertinent Figure, while the buffer recipe and equipment used are shown below.

\section{Reagents}

10x PCR buffer

$250 \mathrm{mM}$ TRIS- $\mathrm{HCl} \mathrm{pH} 8.3$

$500 \mathrm{mM}$ Potassium chloride

1\% Tween 20

$1 \mathrm{mg} / \mathrm{ml}$ gelatine

Magnesium chloride added separately

10x Taq2000 buffer (Stratagene proprietary buffer)

10x Pfu polymerase buffer (Stratagene)

$100 \mathrm{mM}$ Potassium chloride

$100 \mathrm{mM}$ Ammonium sulphate

$200 \mathrm{mM}$ TRIS-HCl pH8.75

$20 \mathrm{mM}$ Magnesium sulphate

1\% Triton X-100

$1 \mathrm{mg} / \mathrm{mlBSA}$

10x KlenTaq buffer

400mM Tricine- $\mathrm{KOH}$ (pH9.2)

$150 \mathrm{mM}$ Potassium Acetate

$35 \mathrm{mM}$ Magnesium acetate 
$750 \mathrm{ug} / \mathrm{ml} \mathrm{BSA}$

Pfu polymerase (Stratagene)

AmpliTaq (Perkin Elmer)

Taq2000 (Stratagene)

Advantage KlenTaq (Clontech)

Marathon-Ready cDNA, Human brain (Clontech)

Primers were dissolved in $10 \mathrm{mM}$ TRIS- $\mathrm{HCl}$, Sterile ' $\mathrm{Q}$ ' water (18M $\Omega$ ) was used in all buffers and reagents were autoclaved. PCR reactions were covered with $\sim 40 \mu$ l of mineral oil (Sigma) and spun briefly at maximum speed to ensure complete mixing of reagents. PCR reactions were carried out in $0.5 \mathrm{ml}$ thin-walled PCR tubes (Alpha labs) in a Perkin Elmer Thermal cycler model 480.

\subsubsection{Direct PCR of bacterial colonies}

\section{Reagents}

$10 \mu l$ pipette tip

$200 \mu l$ pipette tip

L-broth $50 \mu \mathrm{g} / \mathrm{ml}$ ampicillin (per liter

$10 \mathrm{~g}$ bacto-typtone

$5 \mathrm{~g}$ bacto-yeast extract

$10 \mathrm{~g}$ Sodium Chloride

The $\mathrm{pH}$ was adjusted to 7.0 with $\mathrm{NaOH}$ and autoclaved, when cool ampicillin was added to a final concentration of $50 \mu \mathrm{g} / \mathrm{ml}$.

LB agar $/ 50 \mu \mathrm{g} / \mathrm{ml}$ ampicillin

$15 \mathrm{~g} /$ liter bacto agar was added to L-broth and autoclaved; when cool $\left(\sim 45^{\circ} \mathrm{C}\right)$ ampicillin was added to a final concentration of $50 \mu \mathrm{g} / \mathrm{ml}$.

Sterile $Q$ water

\section{Protocol}

Thin walled PCR tubes were labelled and charged with $10 \mu$ sterile $Q$ water: for PCR and liquid culture, a sterile $10 \mu$ lip was fitted on to the end of a 
$200 \mu l$ tip and used to pick a bacterial colony. The $10 \mu$ tip was dipped into the $Q$ water then dropped in to L-Broth $/ 50 \mu \mathrm{g} / \mathrm{ml}$ ampicillin and incubated overnight, shaking at $37^{\circ} \mathrm{C}$. The $200 \mu$ lip was refitted with a fresh $10 \mu l$ tip and the process repeated.

For PCR and solid culture: PCR tubes were prepared as before, colonies were picked with a $200 \mu \mathrm{l}$ tip, which was dipped in to $Q$ water then remaining bacteria were dabbed on to a gridded LB-agar plate (LB-agar $+50 \mu \mathrm{g} / \mathrm{ml}$ ampicillin) and incubated at $37^{\circ} \mathrm{C}$ overnight.

The PCR tubes were sealed and incubated (in a thermal cycler) at $100^{\circ} \mathrm{C}$ for 10 minutes, the other PCR reagents were then added and cycling commenced as normal.

\subsubsection{Cycle sequencing}

\section{Reagents}

Terminator ready reaction mix (ABI)

DNA template

Sequencing primer

Mineral oil (Sigma)

Absolute ethanol

3M Sodium Acetate $\mathrm{pH} 4.6$

$70 \%$ ethanol

\section{Protocol}

All sequencing was performed with the $A B I P R I S M$ dye terminator system; $8 \mu \mathrm{l}$ of terminator ready reaction mix was mixed with $300-500 \mathrm{ng}$ of template DNA, $3.2 \mathrm{pmol}$ of sequencing primer and distilled water was added to a final volume of $20 \mu \mathrm{l}$. The reaction was covered with mineral oil and thermal cycled through the following program $\left(96^{\circ} \mathrm{C}, 30 \mathrm{sec} ; 50^{\circ} \mathrm{C}, 15 \mathrm{sec} ; 60^{\circ} \mathrm{C} 4 \mathrm{~min}\right) \times 25$. The $20 \mu$ reaction was separated from the oil and ethanol precipitated by the addition of $50 \mu \mathrm{l}$ absolute ethanol and $2 \mu \mathrm{l} 3 \mathrm{M}$ Sodium acetate.. The mixture was incubated on ice 
for 10 minutes prior to spinning in a mirocentrifuge at $14,000 \mathrm{rpm}(15,800 \mathrm{xg})$ for 30 minutes,. The supernatant was removed and replaced with $250 \mu 170 \%$ ethanol, then the reactions were respun at 14,000rpm for 5 minutes. The supernatant was discarded and the reactions dried in a vacuum centrifuge, prior to submission to the PNAC laboratory for DNA sequencing.

\subsubsection{Preparation of a circular genomic DNA library for inverse PCR}

\section{Reagents}

High molecular weight genomic DNA (see Section 2.2.6)

Mbol (Promega)

Taql (Promega)

Buffer C x1

$50 \mathrm{mM}$ Sodium chloride

$10 \mathrm{mM}$ TRIS-HCl pH7.9

$10 \mathrm{mM}$ Magnesium chloride

$1 \mathrm{mM}$ DTT

Buffer E x1

$100 \mathrm{mM}$ Sodium chloride

$6 \mathrm{mM}$ TRIS-HCl pH7.5

$6 \mathrm{mM}$ Magnesium chloride

$1 \mathrm{mM}$ DTT

$5 x$ ligation buffer (Gibco)

$250 \mathrm{mM}$ TRIS-HCl pH 7.6

$50 \mathrm{mM}$ Magnesium chloride

$5 \mathrm{mM}$ ATP

$25 \%$ polyethylene glycol

T4 Ligase (Gibco)

\section{Protocol}

Two circular genomic libraries were prepared using 100 units of either Mbol or Taql to digest $70 \mu \mathrm{g}$ of high molecular weight genomic DNA (section 
2.2.6)in a final volume of $700 \mu \mathrm{l}$ over a period of 5 hours. The two protocols were identical except for digestion conditions:-

$\begin{array}{lll}\text { Enzyme } & \text { Buffer } & \text { Temperature of digest } \\ \text { Mbol } & \text { Promega Buffer C } & 37^{\circ} \mathrm{C} \\ \text { Taql } & \text { Promega Buffer E } & 65^{\circ} \mathrm{C} \text { (under oil) }\end{array}$

A $200 \mu \mathrm{l}$ aliquot of the digested DNA was phenol-chloroform extracted, ethanol precipitated and redissolved in $50 \mu$ l of TE, $5 \mu$ l of which was added to $781 \mu \mathrm{l}$ distilled water, $200 \mu \mathrm{l}$ of $5 \mathrm{x}$ ligation buffer, 14 units of T4 ligase and incubated at $4^{\circ} \mathrm{C}$ overnight. The ligated DNA was then ethanol precipitated and redissolved in $100 \mu \mathrm{l}$ of $10 \mathrm{mM}$ TRIS- $\mathrm{HCl} \mathrm{pH} 8.0$, for use as a template in the inverse PCR reaction using the conditions described in Section 5.2.2 and 2.3.1.

\subsubsection{Synthesis of CDNA for 3' RACE and RT-PCR}

\section{Reagents}

Pig brain poly-A RNA (Gift from D. Read)

$1 \mathrm{mg} / \mathrm{ml}$ poly-dT 18 or BAT39 synthesis primers

BAT39 = 5' GTTACGGCTGGATGAGTGACTTTTTTTTTTTTTTTTTTTT 3'

$5 \times 1^{\text {st }}$ strand buffer (Gibco)

250mM TRIS- $\mathrm{HCl}$ pH8.3

$375 \mathrm{mM}$ Potassium chloride

$15 \mathrm{mM}$ Magnesium chloride

$100 \mathrm{mM}$ DTT (Gibco)

$20 \mathrm{mM}$ dNTP (Gibco)

1 volume of $25 \mathrm{mM}$ dATP

1 volume of $25 \mathrm{mM}$ dTTP

1 volume of $25 \mathrm{mM}$ dCTP

1 volume of $25 \mathrm{mM}$ dGTP

1 volume DEPC treated water (Section 2.3.6)

Superscript II reverse transcriptase (Gibco) 
RNase A (Stratagene)

New sterile plastic wear were used throughout the protocol

\section{Protocol}

Two to three micrograms of pig brain poly-A RNA was added to either $0.5 \mu \mathrm{g}$ of poly- $-\mathrm{dT}_{18}$ or BAT39 primer and was made to a final volume of $11 \mu \mathrm{l}$ with DEPC-treated sterile $Q$ water. The mixture was incubated at $70^{\circ} \mathrm{C}$ for 10 minutes, chilled on ice and then $6 \mu \mathrm{l}$ of $5 \times 1^{\text {st }}$ strand buffer, $3 \mu \mathrm{l} 100 \mathrm{mM}$ DTT, $1.5 \mu \mathrm{g} 20 \mathrm{mM}$ dNTP and 1unit of Gibco Superscript II reverse transcriptase were mixed and DEPC-treated water was added to a final volume of $30 \mu l$. The reaction mix was incubated for 90 minutes at $42^{\circ} \mathrm{C}$, then terminated by heating to $95^{\circ} \mathrm{C}$ for 5 minutes. The RNA template was then destroyed by the addition of 150ng RNase A. Product quality was assessed by PCR with a defined pair 0 f primers directed to a low abundance transcript (see Chapter 4 for details).

\subsubsection{DEPC treatment of reagents and equipment}

\section{Reagents}

Diethyl pyrocarbamate (DEPC; Sigma)

\section{Protocol}

Sufficient DEPC was added for a final concentration of $0.1 \%(\mathrm{v} / \mathrm{v})$, the bottle was sealed and stirred overnight at room temperature. The buffer was autoclaved to destroy residual DEPC. Most buffers can be treated excluding ones containing TRIS which prematurely breaks down the DEPC. 


\subsubsection{Amplification of CDNA bacteriophage library for use as PCR template}

\section{Reagents}

L-broth/MgSO 4 /Maltose

$10 \mathrm{~g}$ bacto-typtone

$5 \mathrm{~g}$ bacto-yeast extract

$10 \mathrm{~g}$ Sodium Chloride

$10 \mathrm{mM}$ Magnesium sulphate

The $\mathrm{pH}$ was adjusted to 7.0 with $\mathrm{NaOH}$, autoclaved and allowed to cool prior to the addition of $0.2 \%$ Maltose.

Top agar

$10 \mathrm{~g}$ bacto-typtone

$5 \mathrm{~g}$ bacto-yeast extract

$10 \mathrm{~g}$ Sodium Chloride

$10 \mathrm{mM}$ Magnesium sulphate

$7.5 \mathrm{~g}$ bacto-agar

The $\mathrm{pH}$ was adjusted to 7.0 with $\mathrm{NaOH}$, autoclaved and allowed to cool prior to the addition of $0.2 \%$ Maltose.

SM buffer

$100 \mathrm{mM}$ sodium chloride

$17 \mathrm{mM}$ Magnesium sulphate

$50 \mathrm{mM}$ TRIS-HCl pH 7.5

$0.01 \%$ gelatine

\section{Protocol}

An overnight culture of bacterial strain $\mathrm{C600}-\mathrm{hfl}$ was used to inoculate an L-broth/MgSO4/maltose culture, shaking at $200 \mathrm{rpm}$ at $37^{\circ} \mathrm{C}$ until the $O D_{600}$ reached between 1.8 and 2 . The cells were then spun down at 3000rpm $(1800 \mathrm{xg})$ for 20 minutes in a bench top centrifuge, the supernatant discarded and the pellet resuspended in an equal volume of $10 \mathrm{mM}$ Magnesium sulphate to produce the competent cell stock. 
Final dilution's of the phage library of between $10^{-5}$ and $10^{-10}$ were added to $200 \mu \mathrm{l}$ competent cells and the phage allowed to bind to the bacteria for 15 minutes at $37^{\circ} \mathrm{C}$. Then $2-3 \mathrm{ml}$ of molten top agar at $48^{\circ} \mathrm{C}$ was added and the mix poured on top of a $10 \mathrm{~cm} \mathrm{L-agar} \mathrm{plate} \mathrm{and} \mathrm{allowed} \mathrm{to} \mathrm{set} \mathrm{for} 20$ minutes; the plates were then incubated overnight at $37^{\circ} \mathrm{C}$. The number of plaques was assessed and the dilution that produced near confluent plaques across the top agar was selected. The transfection was then scaled up to grow at least $10^{6}$ bacteriophage plaques, when they grew to about $1 \mathrm{~mm}$ in diameter the plates were transfered to a cold room $\left(4^{\circ} \mathrm{C}\right)$ and $10 \mu l$ of $S M$ buffer per $\mathrm{cm}^{2}$ of petri dish was poured on to the plates and gently rocked overnight. In the morning the SM buffer was poured off, pooled and chloroform was added to a final concentration of $5 \%$. This was shaken then the chloroform and cell debris removed by centrifugation $3000 \mathrm{rpm}(1800 \mathrm{xg})$ in a benchtop centrifuge for 10 minutes. The supernatant was decanted and more chloroform was added to a final concentration of $0.3 \%$, the amplified library was titered as before to determine the number of plaque forming units (pfu) $/ \mathrm{ml}$ and the bacteriophage were stored in the short term at $4^{\circ} \mathrm{C}$ and for long term storage were frozen at $-70^{\circ} \mathrm{C}$. 


\subsection{Hybridisation screening}

\subsubsection{Transfer of bacterial or bacteriophage colonies to solid support}

Reagents

Hybond $\mathrm{N}+$ membranes in $15 \mathrm{~cm}$ disks (Amersham)

$10 \%$ SDS solution

Denaturing solution

$0.5 \mathrm{M}$ Sodium hydroxide

$1.5 \mathrm{M}$ Sodium chloride

Neutralising solution

$0.5 \mathrm{M}$ TRIS- $\mathrm{HCl} \mathrm{pH} 7.4$

1.5M Sodium Chloride

$20 \times S S C$

$3 \mathrm{M}$ Sodium chloride

$0.3 \mathrm{M}$ Sodium citrate

$\mathrm{pH}$ was adjusted to 7.0 with $\mathrm{NaOH}$

3MM paper (Whatman)

\section{Protocol}

Bacterial colonies or bacteriophage were grown over night on agar plates under the appropriate conditions until colonies were $\sim 1 \mathrm{~mm}$ in diameter. Three trays were then lined with $3 \mathrm{MM}$ paper which was saturated with either $10 \%$ SDS, Denaturing solution or Neutralising solution. The Hybond $\mathrm{N}+$ disk was labelled in soft pencil then laid on top of the agar and left in place for 1 minute, 4 to 6 holes were punched asymmetrically in the membrane using a $19 \mathrm{G}$ needle dipped in permanent black ink to register the position of the membrane on the agar.

Then membrane was then laid colony side up, on to the SDS soaked 3MM paper for 3 minutes. It was then similarly transfered to the denaturing solution soaked paper and exposed for 5 minutes. Finally, it was exposed to neutralising buffer-soaked paper for 5 minutes. The membrane was rinsed briefly by dipping in a bath of $2 \times S S C$, then laid on dry $3 \mathrm{MM}$ paper for 30 minutes until dry. The 
DNA was fixed to the membrane by baking for 2 hours at $80^{\circ} \mathrm{C}$ and stored dry until needed.

\subsubsection{Southern Blotting}

\section{Reagents}

Hybond $\mathrm{N}+$ membranes in $15 \mathrm{~cm}$ disks (Amersham)

Denaturing solution

$0.5 \mathrm{M}$ Sodium hydroxide

1.5M Sodium chloride

Neutralising solution

$0.5 \mathrm{M}$ TRIS-HCl pH 7.4

1.5M Sodium Chloride

$20 \times S S C$

3M Sodium chloride

$0.3 \mathrm{M}$ Sodium citrate

$\mathrm{pH}$ was adjusted to 7.0 with $\mathrm{NaOH}$

\section{Protocol}

The agarose gel was immersed with gentle rocking in several gel volumes of denaturing solution for $\mathbf{4 5}$ minutes. The gel was then rinsed in distilled water and immersed in neutralising buffer for $\mathbf{3 0}$ minutes, at which point the buffer was changed for fresh neutralising buffer and the gel incubated for a further 15 minutes. The gel was placed upside down on a raised platform on top of two oblong strips of Whatman 3MM paper cut to the width of the gel, wetted in 10xSSC with the ends of the oblong immersed in a tray of 10xSSC (approximately 1 litre). Taking care no bubbles were trapped between layers, the edges of the gel were masked with Saranwrap so that the paper towels (see below) would not contact the 10xSSC pool or the wetted 3MM paper. A piece of Hybond $\mathrm{N}+$ membrane (which had been soaked in distilled water for 5 minutes) was placed on top of the gel and small holes were punched with a needle to mark the position of the sample origin. The Hybond was covered with two sheets of 
3MM paper cut to fit the gel and this was covered with a 5-8 $\mathrm{cm}$ stack of paper towels surmounted by a $500 \mathrm{~g}$ weight. This was left overnight for the DNA to transfer after which the Hybond $\mathrm{N}+$ membrane was removed from the stack and fixed by baking at $80^{\circ} \mathrm{C}$ for two hours. The remains of the agarose gel were sometimes restained by immersion in $0.5 \times T B E$ and $0.5 \mu \mathrm{g} / \mathrm{ml}$ ethidium bromide (see section 2.2.1) and checked under UV light to ensure the DNA had fully transfered.

\subsubsection{Labeling DNA probes}

\section{Reagents}

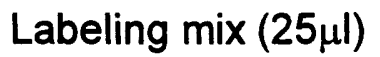

1x PCR buffer

$150 \mu \mathrm{M} d[\mathrm{GAT}]$ (2.5mM of dATP, dGTP, and dTTP)

$2 \mathrm{mM}$ Magnesium chloride

0.5u AmpliTaq (Perkin Elmer)

$5 \mu \mathrm{l}\left[{ }^{32} \mathrm{P}\right] \mathrm{dCTP}$ labelled to $>3000 \mathrm{Ci} / \mathrm{mmole} ; 10 \mathrm{mCi} / \mathrm{ml}$ (Amersham)

PCR-produced DNA

Labeling primer

\section{Protocol}

All the DNA used as hybridisation probes were available as PCR products and were labelled by single strand PCR; the labelling mix above was prepared and $20 \mathrm{ng}$ of the PCR product to be labelled was added along with $0.2 \mu \mathrm{M}$ of forward primer for the product. The mix was covered with mineral oil and thermal cycled $\left(94^{\circ} \mathrm{C} 5 \mathrm{~min},\left[94^{\circ} \mathrm{C} 30 \mathrm{sec} ; 55^{\circ} \mathrm{C} 30 \mathrm{sec} ; 72^{\circ} \mathrm{C} 1 \mathrm{~min}\right] \times 10\right)$. The labelled DNA was then purified by the QIAspin PCR purification kit (Section 2.2.3) and the labelling judged by measuring the counts per second of a $1 \mu$ aliquot in a scintillation counter. 


\subsubsection{Hybridisation conditions}

\section{Reagents}

Hybridisation buffer

$6 \times$ SSC

$0.5 \%$ SDS

1x Denhardts solution

$100 \mu \mathrm{g} / \mathrm{ml}$ denatured fragmented salmon sperm DNA (Sigma)

50x Denhardts solution (per liter)

10g Ficol (Type 400, Pharmacia)

$10 \mathrm{~g}$ polyvinylpyrrolidone (Pharmacia)

$10 \mathrm{~g} \mathrm{BSA}$ (Sigma)

20xSSC

3M Sodium chloride

$0.3 \mathrm{M}$ Sodium citrate

pH was adjusted to 7.0 with $\mathrm{NaOH}$

\section{Protocol}

Both probes used in this project were relatively short (<200bp) and identical conditions were used in both experiments.

The membrane was incubated with $10 \mathrm{ml}$ of hybridisation buffer for 1 hour at $65^{\circ} \mathrm{C}$ in a Techne hybridisation oven, then approximately $10^{5} \mathrm{cpm}$ of labelled probe per $\mathrm{cm}^{2}$ of membrane was boiled for 1 minute and applied to the membrane and allowed to hybridise for at least 1 hour at the same temperature.

The membranes were washed in $2 \times S S C+0.05 \%$ SDS at $65^{\circ} \mathrm{C}$ changing the buffer every 20 minutes for 80 minutes. The membrane was then washed in $0.2 \times S S C+0.05 \%$ SDS for $2-5$ minutes at $65^{\circ} \mathrm{C}$. The membrane was monitored with a hand-held Geiger counter throughout the washing process and if the radioactivity dropped below $10 \mathrm{cps}$ or the membrane had been washed in $0.2 \times S S C$, the washing was stopped and the membrane wrapped in Sarenwrap and autoradiographed using Biomax MS film (Kodak) with intensifying screens. 


\section{Chapter 3 Isolation and protein sequencing of NTE}

\subsection{Introduction}

The original aim of this project was to clone the NTE gene from chicken brain CDNA. The chicken has long been the test animal of choice for the study of Organophosphate Induced Delayed Polyneuropathy (OPIDP), since it is apparently more sensitive to OPIDP and due to its bipedal gait clinical symptoms are pronounced and thus can be easily and accurately assessed (Abou-Donia, 1981). Translation of the chicken CDNA sequence would allow synthesis of NTE peptides to which antibodies could be raised for use in immunohistochemistry. In addition chicken brain is known to be relatively rich in NTE compared with several mammalian species (for example pig and sheep brain have $20 \%$ less NTE and rat has $65 \%$ less; Meredith and Johnson, 1989).

The NTE purification protocol depends on the novel biotinylated organophosphate S9B (1,[Saligenin cyclic phosphoryl],-9,-biotinyl-diaminonoane; Figure 3.1). Incubation of S9B with brain microsomes leads to a specific labelling of NTE at the active site serine residue, producing a biotinyl-NTE complex which can be easily separated from almost all other proteins by avidin's extraordinary affinity for biotin (Glynn et al, 1994). 


\subsection{Results and Discussion}

\subsubsection{Chicken brain NTE: Purification and protein sequencing}

A typical preparation of chicken brain NTE is summarised in Figure 3.2 and described in detail in Sections 2.1.1 to 2.1.6. Chicken brains were homogenised in TE containing $1 \mathrm{mM}$ DTT and the microsomes were prepared by differential centrifugation. The pelleted microsomes were resuspended in $0.9 \%$ $\mathrm{NaCl} / \mathrm{TE}$ and stored at $-20^{\circ} \mathrm{C}$ while the protein concentration of a sample was assayed using the BioRad DC reagent.

The microsomal protein solution was thawed and diluted to a protein concentration of $2 \mathrm{mg} / \mathrm{ml}$ with $0.9 \% \mathrm{NaCl} / \mathrm{TE}$ and $\mathrm{S} 9 \mathrm{~B}$ added to a final concentration of $160 \mathrm{nM}$. After an incubation of 20 minutes at $37^{\circ} \mathrm{C}$ the microsomes were washed to remove unreacted $\mathrm{S} 9 \mathrm{~B}$, then solubilized by boiling for 10 minutes in TE buffer plus $0.15 \%$ SDS, $0.1 \%$ DTT.

After a brief spin $(2000 \mathrm{xg} ; 10 \mathrm{~min})$ the clear extract was passed through an avidin-Sepharose affinity column, and after washing, the biotinyl-NTE complex subsequently eluted by boiling the avidin-Sepharose slurry in $0.25 \%$ SDS, $0.25 \%$ DTT in 0.1 XTE buffer (Figure 3.3). This avidin eluate was concentrated using a Centrispin 100 filter prior to preparative SDS-PAGE, which removed the endogenous biotinylated protein present in the avidin eluate (Figure 3.4).

A total of $200 \mu \mathrm{g}$ of pure chicken NTE was prepared from $1.8 \mathrm{~kg}$ of fast frozen chicken brain using the methods described above. Of this pure NTE, $60 \mu \mathrm{g}$ was used as part of a group effort to produce a mouse monoclonal antibody. The remaining $140 \mu \mathrm{g}$ was used in protein sequencing experiments.

NTE has proven to be far from an ideal subject for peptide sequencing. Intact NTE protein was resistant to $\mathrm{N}$-terminal sequencing by Edman degradation. Furthermore, CNBr cleavage, acid hydrolysis, chymotrypsin, thermolysin or 
trypsin digestion were unsuitable for NTE digestion as they produced no discrete bands on SDS-PAGE which could be extracted and sequenced. Only partial digestion with Staphylococcus aureus V8 protease (EC:3.4.21.9) produced a selection of fragment bands with sizes of between 4 and $70 \mathrm{kDa}$ which were suitable for peptide sequencing.

Small scale experiments showed that the optimum digestion conditions were $1 \mu \mathrm{g}$ of V8 protease to $10 \mu \mathrm{g}$ of NTE, digesting for 2 hours at $37^{\circ} \mathrm{C}$. The 140 $\mu \mathrm{g}$ of NTE was divided into several batches, digested and then submitted to the Protein and Nucleic Acid (PNAC) sequencing laboratory. Digested fragments were resolved on a $16.5 \%$ Tris-Tricine gel and Western blotted on to Immobilon-P membrane (Figure 3.5). Then individual bands were excised and sequenced by Edman degradation.

A total of five bands were cut from the Immobilon-P blots: of these, only three produced peptide sequences (this sort of peptide blockage was also experienced in sequencing pig NTE). A further four sequences had been produced in previous work by other members of the group (Figure 3.6). The sequence data produced by this work were not only heterogeneous but also the amount of derivatized amino acid produced by the Edman degradation was low. Thus, even when the sequencing indicated a single residue, there was a certain amount of suspicion as to its accuracy because the sequencing was operating at the limits of its sensitivity. These ambiguities severely limited the usefulness of the sequence data, as degenerate oligonucleotide probes and primers must be based on reliable sequence. This is because the specificity of a probe is related to its length and since the length is usually short (typically 21 bp or 7 residues) a single residue error can cause a large percentage error, further reducing the already limited specificity of a degenerate oligonucleotide pool.

After a few attempts to clone chicken brain NTE CDNA (data not shown) it became clear that producing sufficient reliable protein sequence data would be 
prohibitively expensive and efforts were focused on obtaining pig brain NTE, since the source tissue was readily available at low cost.

\subsubsection{Pig brain NTE: Purification and protein sequencing}

The methods used to obtain pure NTE protein and sequence data from pig brain were identical to those described for chicken. A group effort over a period of 2 years and more than 50 Edman sequencing runs generated reliable $\mathrm{N}$ terminal sequence for 8 NTE peptides from V8 digest fragments (Figure 3.7).

One modification to the purification protocol was developed to produce a cleaner set of V8 digest bands. Products from a V8 digestion of porcine NTE were passed through a second avidin-Sepharose column and eluted to extract the protein fragments bearing the S9B. Both the V8 avidin eluate and the flowthrough were run on SDS-PAGE, blotted and bands were excised for sequencing (Figure 3.8).

Two sequences were found in the V8 avidin eluate: LTNP and XGVP. The LTNP peptide sequence found in the V8 avidin eluate was associated with a $36 \mathrm{kDa}$ peptide fragment and indicated that the previously observed $72 \mathrm{kDa}$ fragment initiated by LTNP would also be S9B biotinylated. In the flowthrough, LTNP was found on a $20 \mathrm{kDa}$ peptide; because this peptide had failed to be bound by avidin, it was thought to be unbiotinylated, putatively placing the active site serine less than $36 \mathrm{kDa}$ and more than $20 \mathrm{kDa}$ from the LTNP sequence. The XGVP peptide sequence in the V8 avidin eluate was found on $32 \mathrm{kDa}$ and $23 \mathrm{kDa}$ fragments. In the flowthrough, XGVP was found on a $16 \mathrm{kDa}$ fragment, so was also thought to be unbiotinylated. This tentatively placed the active site serine less than $23 \mathrm{kDa}$ and more than $16 \mathrm{kDa}$ from the XGVP sequence. These sequence data were used to assemble a tentative peptide map around the active site serine which will be discussed in greater detail in Chapter 5 (Figure 5.1 and Section 5.1). 
The final batch of peptide sequences were produced by excising V8 digested NTE fragments from a Western blot. A portion of each band was Nterminally sequenced, and the rest was redigested with trypsin. The trypsin digested fragments were fractionated by HPLC and then sequenced. This work (performed by Dr Tony Willis of the MRC Immunochemistry Unit, Oxford) produced one familiar sequence (DLGL) and five novel ones (Figure 3.9). The original aim of this work was to provide fresh sequence with some reliable evidence as to their relative positions within the NTE protein, to aid in design of degenerate PCR reactions for further cloning attempts. However it was found that DGHL, WKS, FDQI and DLGL all had near perfect matches with sequences in the Expressed Sequence Tag (EST) division of EMBL. These sequence matches are discussed further in Chapter 6 (Section 6.1).

\subsection{Significance of NTE peptide sequences in isolating the NTE cDNA}

The pig brain NTE sequences where far more reliable than those produced from chicken NTE. The improvement in sequence quality was essentially due to the larger amounts of NTE it was possible to produce from pig brain. Firstly, it allowed more digested NTE to be submitted to PNAC which gave a stronger signal on the Edman degradation sequencing. Secondly, it made multiple sequencing runs possible and allowed detection of errors in detecting the residue and often resolved ambiguous residues.

Despite the relatively large quantities of pig brain NTE available for analysis, NTE still proved to be a challenging subject for sequencing. For unknown reasons, certain peptide fragments proved to be resistant to repeated Edman sequencing attempts (mirroring the experience with chicken NTE) and furthermore some turned out to be the result of contaminating avidin and carboxylesterase sequences (figure 3.7). 
The avidin sequence contamination was easily detected because avidin's full protein sequence was available from SWISSPROT (sW:P02701) and a simple BLAST search was sufficient to detect any homology. However, the carboxylesterase contamination was considered a particularly insidious threat to the project. Since carboxylases are endogenously biotinylated (PROSITE: PS00188) they are greatly enriched by the avidin-Sepharose affinity protocol (see Figure 3.3). Because the carboxylase contamination was never fully characterised (Glynn et al, 1994), the concern was that the purification protocol could yield peptide sequence derived from a previously unknown carboxylesterase which copurified with NTE. Thus protein sequence which showed any similarity to carboxylase sequences was regarded with suspicion.

The routine database searching using the NTE peptide sequences was also an important part of the overall cloning strategy. Both the EMBL and SWISPROT databases were regularly searched using the BLAST and BLITZ programs. BLITZ was found to be better at detecting similarities between short peptides and database entries but unfortunately it was limited to searching the smaller SWISSPORT database. BLAST was found to be less sensitive when using short peptides; however, it could be used to search EMBL DNA database, comparing the peptide sequence with each DNA sequence translated in all six reading frames.

It was hoped that sequence matches produced by the database searches could assist the project in two ways. First, it was possible that NTE had already been cloned as an EST (See Section 1.2.5); this could be obtained from the IMAGE consortium (Lennon et al, 1996) for further investigation. Secondly, if two NTE peptides were found to be similar to a single protein this could have given the relative position and separation of the NTE peptides; this information could then have been used to design degenerate oligonucelotides which would yield a PCR product of predictable size and only require a limited number of primer combinations to be investigated. 
Up until the beginning of 1997, database searches using the eight older peptide sequences (Figure 3.7 ) had only produced two significant matches (between DLGL, LAKL and YOL4_CAEEL): these are discussed fully in Chapter 6 (Section 6.1). After this date, sequences began to appear linking YOL4_CAEEL with other NTE peptide sequences. Had the tryptic digest/HPLC sequence data been unavailable, either of these similarities would have refocused interest in YOL4 and lead to the cloning of NTE. In February 1997 the yeast sequence YMF9_YEAST (sw:Q04958; Devlin et al, unpublished) appeared in SWISSPROT which had a similarity with WPG (Figure 3.10). YMF9 is a hypothetical $187 \mathrm{kDa}$ protein from Saccharomyces cerevisiae and was listed as being part of the YOL4/YCHK family of hypothetical proteins. YMF9 is discussed more fully in Chapter 7 (Sections 7.2.2 and 7.2.3). In June 1997 a 424 bp zebrafish EST clone 8K14 (em:AA494858) was submitted to EMBL (Clark et al, 1997) listed as being similar to both YOL4 and YMF9. The translated sequence contained two very close matches with both ADLT and WPG (Figure 3.10). The $8 \mathrm{~K} 14$ clone is an EST of unusual length since the sequence match is located within 210 amino acids of the N-terminal end of YOL4 (all the Human NTE ESTs in Chapter 6 are less than 2kbp long) and 8K14 probably represents a near full length NTE-like clone. This would be of great interest since full length sequence of another vertebrate NTE clone could highlight regions of functional importance in NTE, by looking at the regions of similarity between the sequences. 


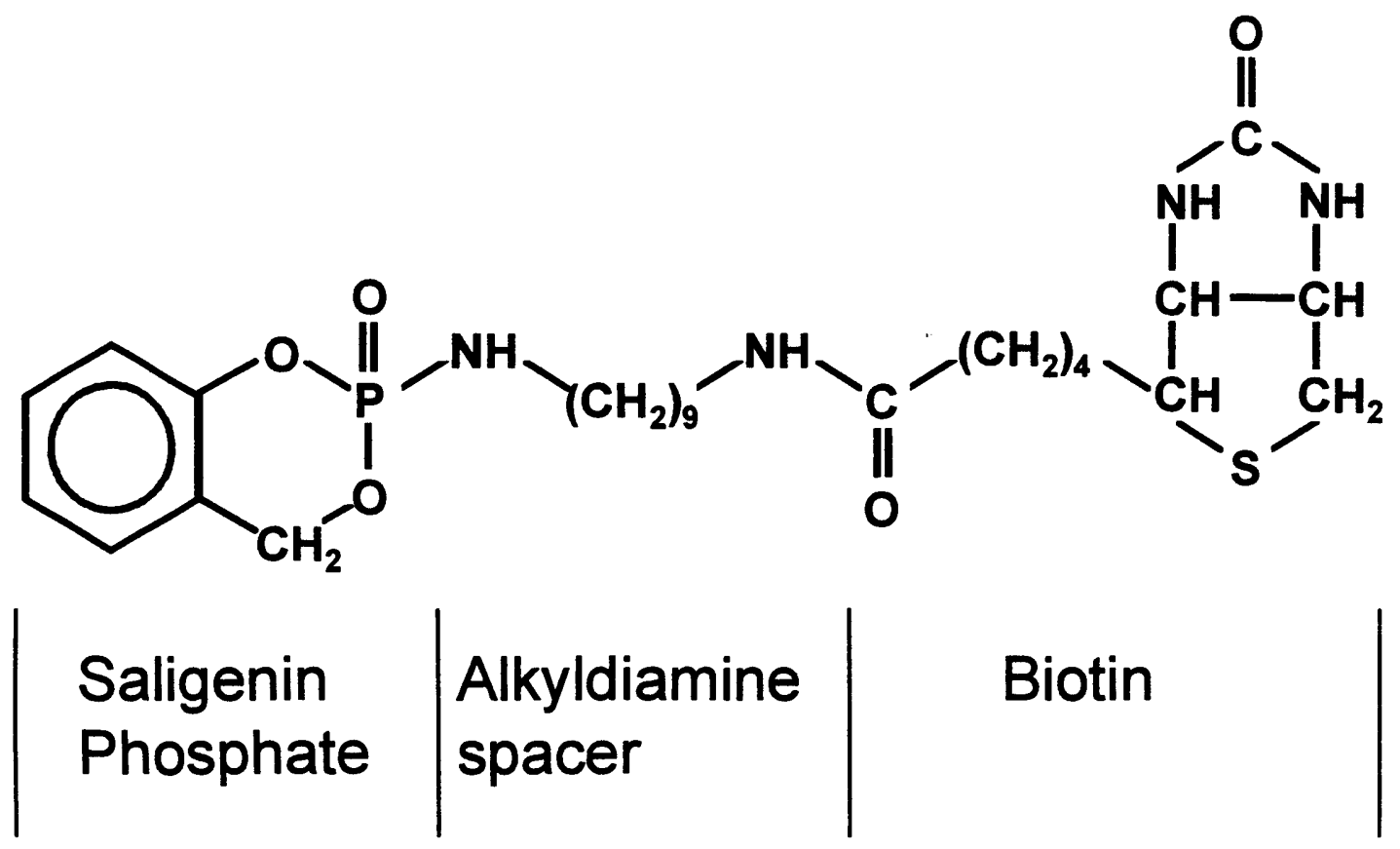

Figure 3.1 Structure of 1, [Saligenin cyclic phosphoryl],-9,-biotinyldiaminonoane (S9B). 


\section{$580 \mathrm{~g}$ of fast frozen chicken brain}

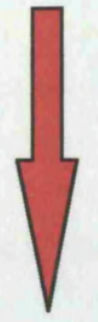

(i) Homogenization of tissue

(ii) Differential centrifugation

\section{$1.5 \mathrm{I}$ microsomes $2 \mathrm{mg} / \mathrm{ml}$ protein concentration}

$$
\text { ( } \approx 900 \mu \mathrm{g} \text { NTE) }
$$

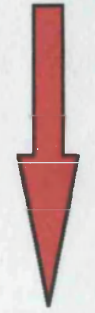

(iii) S9B labeling

(iv) Solubilisation in SDS

(v) Absorbtion/elution from

avidin-Sepharose

\section{Avidin eluate $(\approx 200 \mu \mathrm{g}$ NTE in $30 \mathrm{ml}$ buffer $)$}

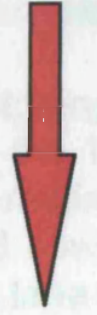

(vi) Concentration of NTE in Centriprep 100

(vii) Preparative PAGE

(viii) Acetone precipitation

\section{Purified NTE ( $\approx 60 \mu \mathrm{g}$ NTE)}

Figure 3.2 Flow chart summarising the purification stages of NTE from chicken brain to purified NTE.

The amount of NTE in the microsomal fraction was estimated from the reported proportion of NTE in microsomal protein $(0.03 \%$; Rüffer-Turner et al, 1992). The other yields of NTE were estimated by comparison with standard quantities of protein on stained SDS-PAGE gels (See Figure 3.3 and Section 2.1.5). 


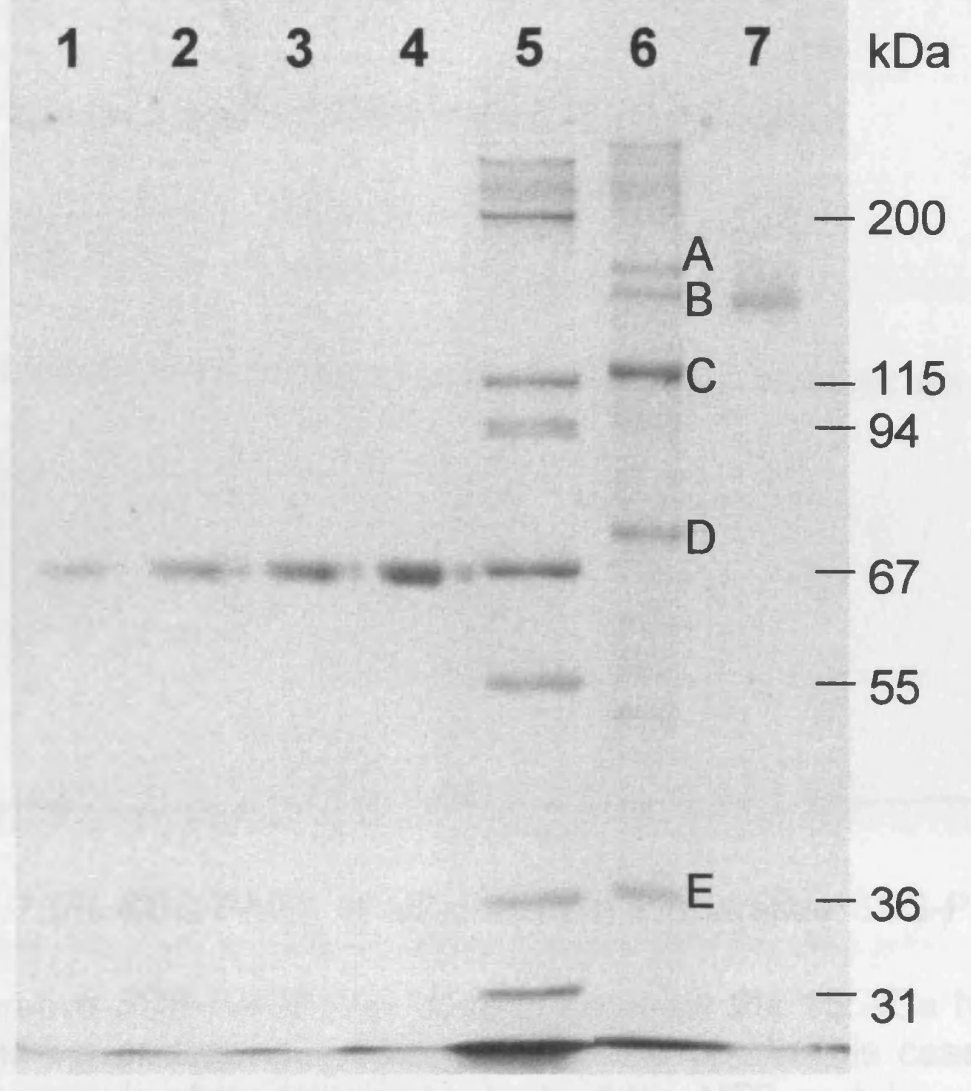

Figure $3.37 .5 \%$ SDS-PAGE illustrating the two stages of NTE purification.

In lane 6 band $B$ represents the 155kDa NTE protein, bands A, C and D are endogenously biotinylated proteins (probably carboxylases). Band $E$ represents a protein that binds S9B however it is not an NTE type activity as it is sensitive to paraoxon treatment. In lane 7 the major band is pure NTE.

$\begin{array}{ll}\frac{\text { Lane }}{1} & \\ 2 & 300 \mathrm{ng} \mathrm{BSA} \\ 3 & 450 \mathrm{ng} \text { BSA } \\ 4 & 600 \mathrm{ng} \text { BSA } \\ 5 & \text { Novex Molecular weight markers } \\ 6 & \text { Avidin eluate } \\ 7 & \text { 155 kDa NTE protein band purified } \\ & \text { by Preparative SDS-PAGE }\end{array}$




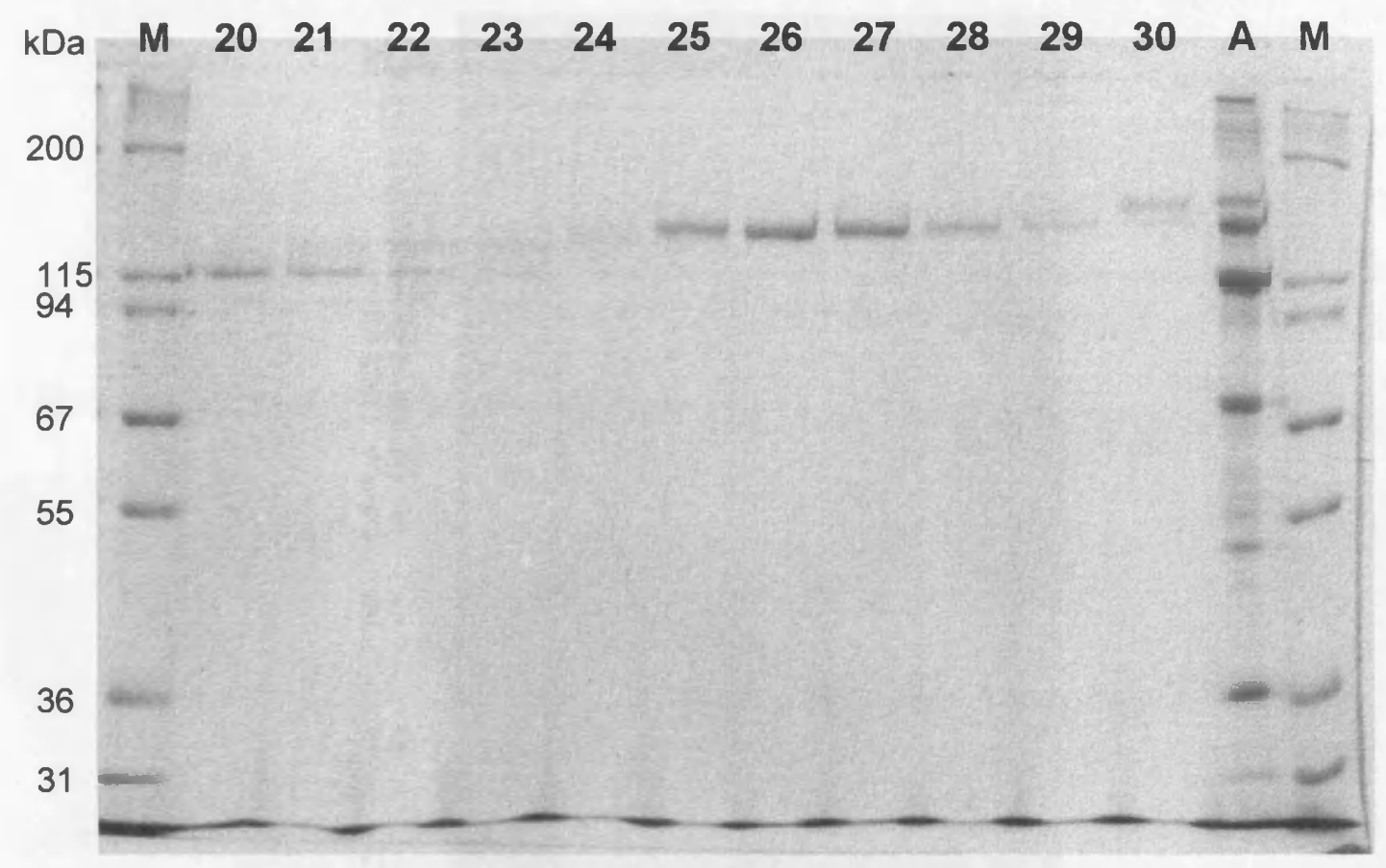

Figure $3.47 .5 \%$ SDS-PAGE of aliquots from Preparative SDS-PAGE of Avidin eluate.

Preparative SDS-PAGE was used to separate the $155 \mathrm{kDa}$ NTE band from the contaminating endogenous biotinylated proteins. In this case fractions 26 , 27 and 28 were judged to contain the bulk of the NTE protein and these three were pooled and acetone precipitated prior to use in sequencing or antibody production.

Careful examination reveals a faint band in lanes 212223 and 24 located between the $120 \mathrm{kDa}$ carboxylase protein and the $155 \mathrm{kDa}$ NTE. In retrospect, knowing that NTE displays multiple splice forms (see Section 6.2.4) it is possible that this band represents an NTE isoform. However it could also represent a carboxylase or a proteolytic cleavage of the $155 \mathrm{kDa}$ NTE: further work would be needed to confirm this speculation.

\footnotetext{
Lane

M Novex Protein Molecular weight markers

A Avidin eluate

20 to 30 Aliquot number of preparative SDS-PAGE fractions
} 


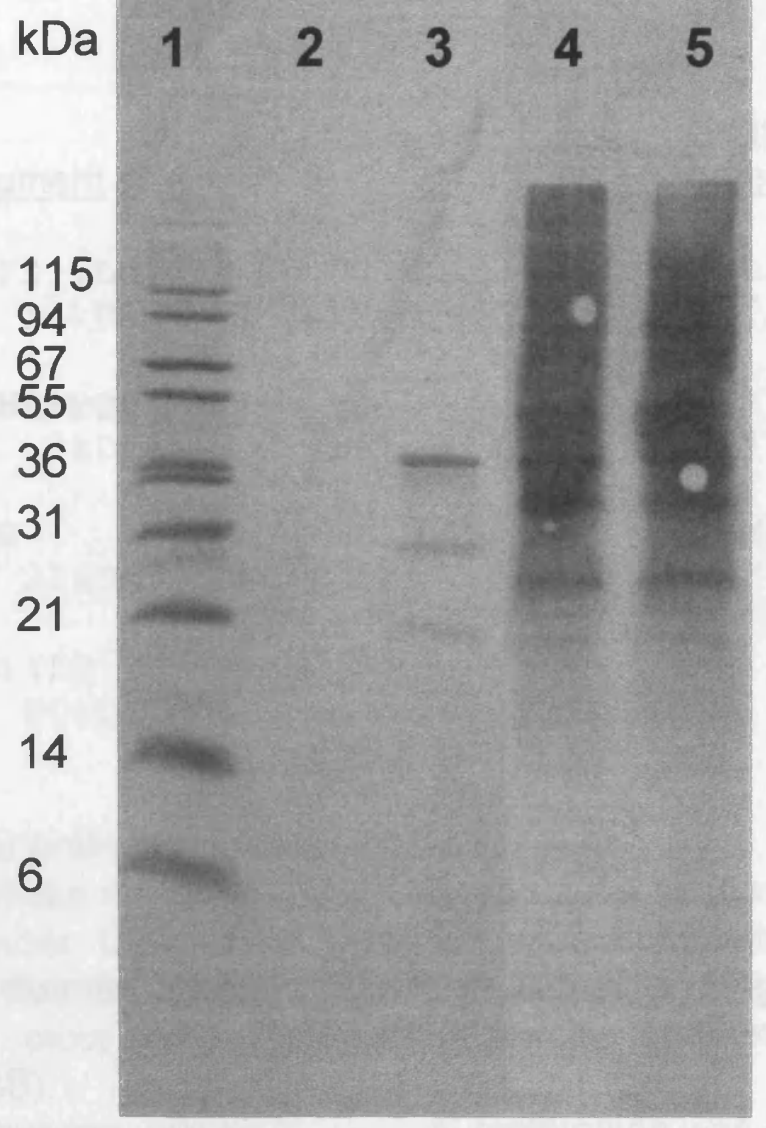

Figure 3.5 V8-digested NTE blotted on to Immobilon P prior to sequencing.

$\begin{array}{ll}\frac{\text { Lane }}{1} & \\ 2 & \text { Novex molecular weight markers } \\ 3 & \text { Blank lane } \\ 4 & \text { V8 protease } \\ 5 & \text { V8 digested NTE }\end{array}$


Name Sequence

date and fragment size

C18A (ML) (QF) (PS) (LH) (RE)

3/12/93 18kDa

C18B (SMKR) QPLRLFPQP

$8 / 11 / 93 \quad 18 \mathrm{kDa}$

C21 GQSAW (PQ) P

$14 / 12 / 93 \quad 21 \mathrm{kDa}$

C10 (AKRS) (DG) (LR) (TI) E (FV) DVAN

$11 / 11 / 93 \quad 10 \mathrm{kDa}$
Name Sequence

date and fragment size

$\begin{array}{lll}\text { C29 VILPEXDR } & \\ & 28 / 3 / 94 \quad 29 \mathrm{kDa}\end{array}$

C18C (SAV) QLALEPQEE

$6 / 4 / 94 \quad 18 \mathrm{kDa}$

C8 (AML) DLTEPD

$29 / 3 / 94 \quad 8 \mathrm{kDa}$

Figure 3.6 Chicken brain peptide sequence.

A total of 7 Edman degradations produced useful sequences from chicken brain NTE. Sequences $\mathrm{C} 29, \mathrm{C} 18 \mathrm{C}$ and $\mathrm{C} 8$ were completed as part of this project. Brackets denote residues detected within a single Edman cycle. Residues shown in colour were duplicated in another sequencing run (C8 and C10; C18A and C18B).

The $\mathrm{C} 18 \mathrm{~B}$ sequence with an $\mathrm{N}$-terminal methionine was likely to be correct since an antibody raised to a synthetic peptide of this sequence proved able to detect chicken brain NTE in a microsomal protein preparation. 


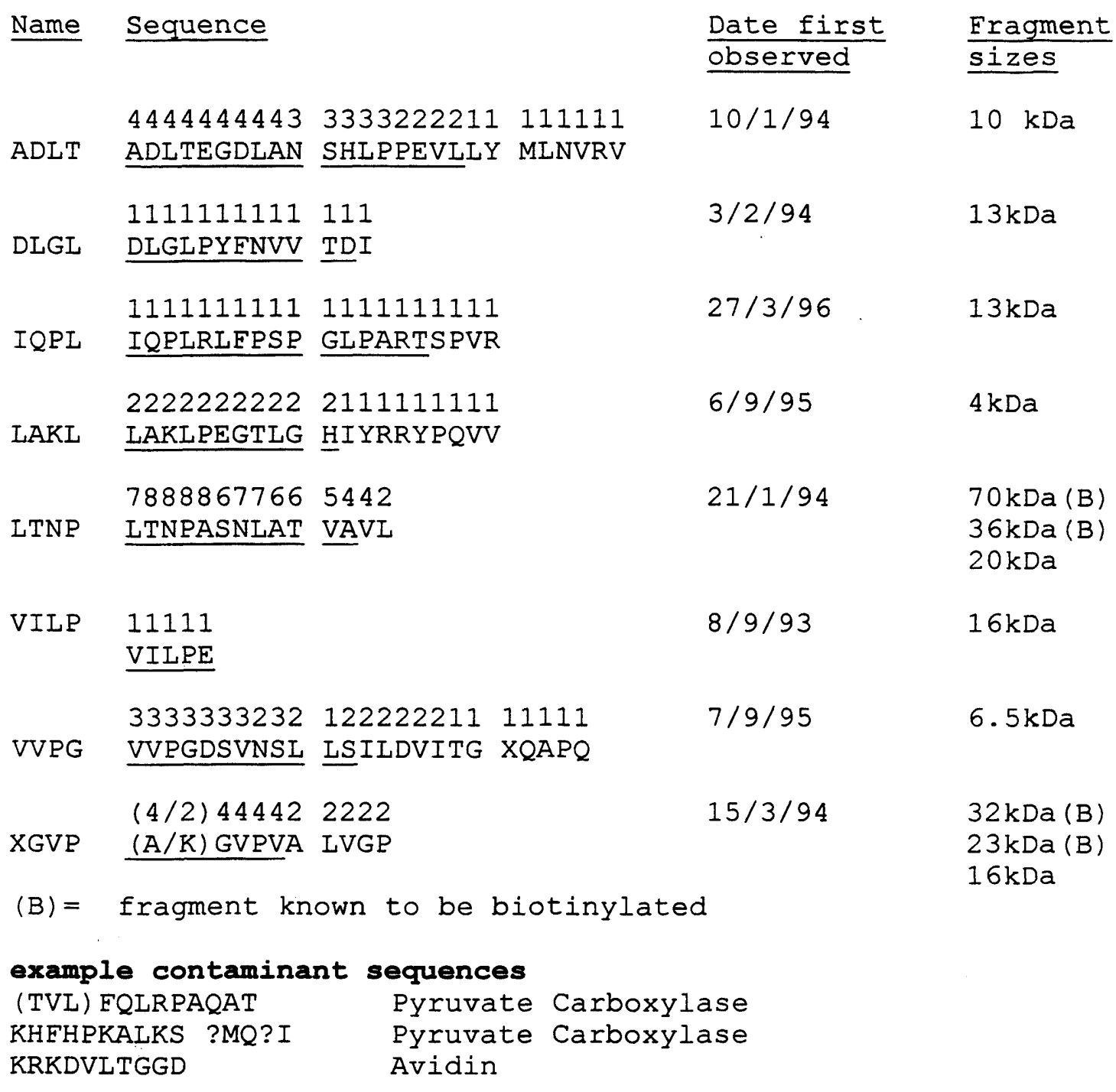

Figure 3.7 Pig NTE peptide sequence data.

This figure summarises over 50 Edman sequencing runs (omitting sequences which were of insufficient quality to use in cloning). The digit above each residue represents the number of times the residue had been observed in that position in repeated protein sequencing runs. Thus ADLT has been observed four times and combines sequences of $9,14,18$ and 26 amino acids. The underlined sequences indicate the extent of reliable sequence suitable for design of oligonucleotide probes. Reliability was assessed by considering the number of sequencing runs that had detected that residue or, if the sequence was unique, the yield of derivatized amino acid produced by the Edman degradation run.

The contaminant sequences were an important factor in the interpretation of the peptide sequence data, since they showed that the 'pure' NTE protein produced by the S9B protocol was susceptible to contamination and thus any protein sequence which had some similarity to any known endogenously biotinylated proteins was regarded with suspicion. 


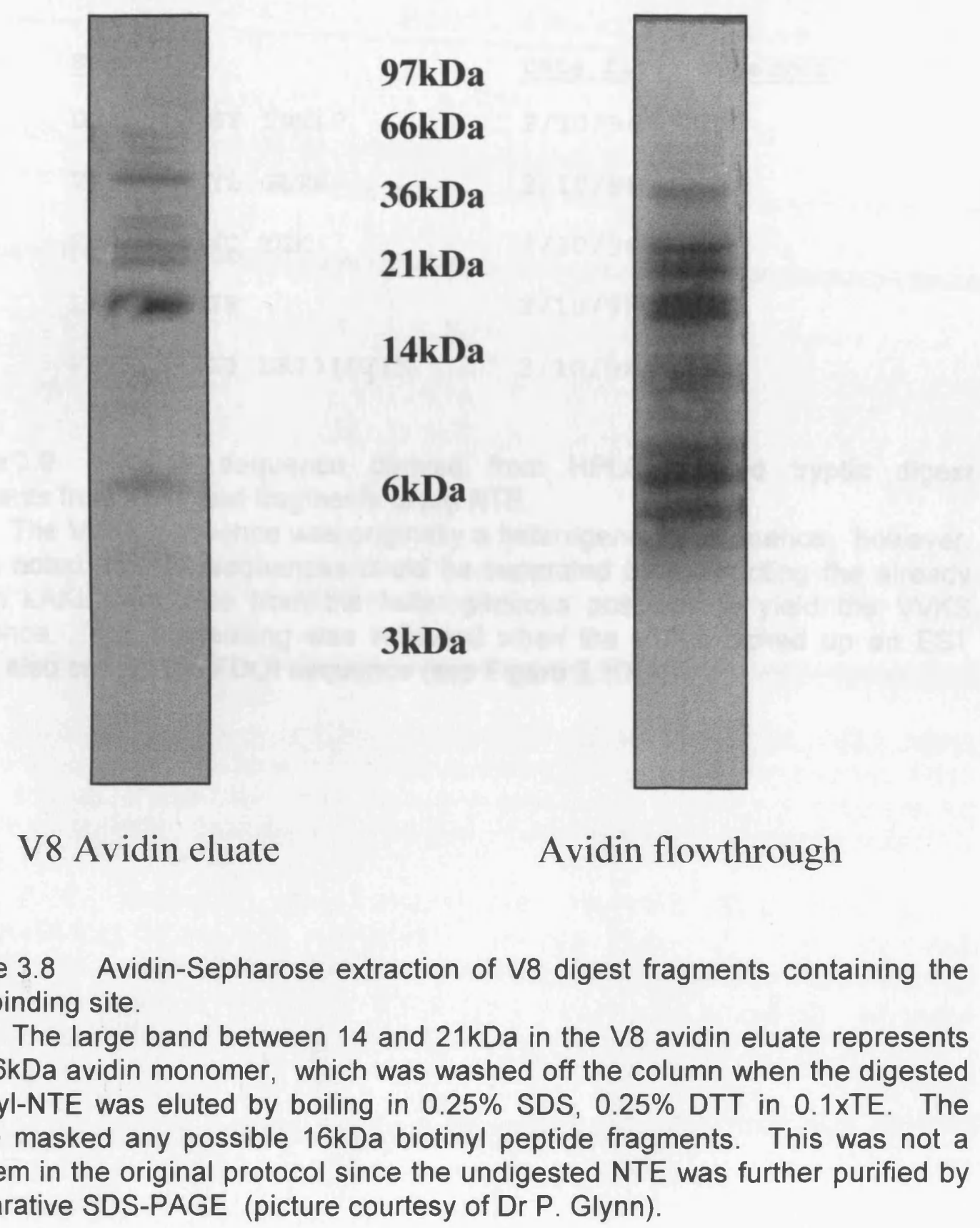

Figure 3.8 Avidin-Sepharose extraction of V8 digest fragments containing the S9B binding site.

The large band between 14 and $21 \mathrm{kDa}$ in the $\mathrm{V} 8$ avidin eluate represents the $16 \mathrm{kDa}$ avidin monomer, which was washed off the column when the digested biotinyl-NTE was eluted by boiling in $0.25 \%$ SDS, $0.25 \%$ DTT in $0.1 \times$ TE. The avidin masked any possible $16 \mathrm{kDa}$ biotinyl peptide fragments. This was not a problem in the original protocol since the undigested NTE was further purified by Preparative SDS-PAGE (picture courtesy of Dr P. Glynn). 


$\begin{array}{lll}\text { Name } & \text { Sequence } & \text { Date first observed } \\ \text { DGHL } & \text { DGHLLTDGGY INNLP } & 2 / 10 / 96 \\ \text { VTFL } & \text { VTFLALHNYL GLTN } & 2 / 10 / 96 \\ \text { FDQI } & \text { FDQIYDVGYQ YGK } & 2 / 10 / 96 \\ \text { LPEG } & \text { LPEGTLGHIK } & 2 / 10 / 96 \\ \text { VVKS } & \text { VVKSSS?DG? LR??IDYFK } & 2 / 10 / 96\end{array}$

Figure 3.9 Peptide sequence derived from HPLC purified tryptic digest fragments from V8 digest fragments of pig NTE.

The WKS sequence was originally a heterogeneous sequence; however, it was noted that the sequences could be separated by subtracting the already known LAKL sequence from the heterogeneous positions to yield the WKS sequence. This processing was validated when the WKS picked up an EST which also carried the FDQI sequence (see Figure 3.10). 
A) $\begin{array}{ccc}\text { VVPG } & 1 \text { VVPGDSVNSLLSILDVITGXQAPQ } & 24 \\ & 11::=11: 111::=1 \quad: \\ \text { YMF9 } & 328 \text { LNTVKPGNPTSLVILNLFTHSMSYGNS } 357\end{array}$

B)

ADLT

1 ADLTEGDLANSHLPPEVL 18

8K14 31 LLEADLTEFDMQNSHLPSEVLYML 55

C)

VVPG 1 VVPGDSVNSLLSILDVITGXQAPQ 24

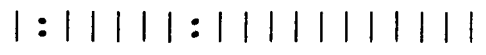

8K14 119 VKHVLPGDSVHSLLSILDVITGYP 142

Figure 3.10 Sequence similarities between ADLT, VPG and sequences linked to YOL4.

A) WPG shows a moderate similarity with the $\mathrm{N}$-terminal domain of YMF9_YEAST (sw:Q04958).

B) ADLT shows a very close match with zebrafish clone 8K14 when translated in reading frame +3 . This homology is of particular interest since ADLT sequence was used to produce a peptide antibody which is known to be specific to pig NTE, thus the ADLT sequence was known to represent a true NTE sequence.

C) WPG shows a close match with zebrafish EST clone 8K14 (em:AA494858) when it is translated in reading frame +2 . The frameshift between $A D L T$ and WPG is unsurprising since the DNA sequence the translation was made from was made from a 'single pass' sequencing run which can easily contain single base errors and frameshifts. 


\section{Chapter 4 Attempts to clone NTE cDNA using Rapid Amplification of cDNA Ends (RACE)}

\subsection{Introduction}

The peptide sequences available at the initiation of the cloning attempt are shown in Figure 4.1. Every sequence except XGVP contained one or more amino acids encoded by highly redundant codons (see Section 1.3.1) and so did not favour design of oligonucleotides for use as specific probes for hybridisation to a CDNA library, or primers for PCR. Furthermore, until near the end of the project there were no clues to the relative position of these peptides within NTE. The general cloning strategies appropriate to cloning NTE and early attempts by other members of this laboratory in this endeavour have been discussed in Chapter 1. This chapter concentrates on the application of 3' RACE to the problem the background to this stratagy is discussed in detail in Section 1.3.4.

\subsection{Results and Discussion}

\subsubsection{Synthesis and quality control of cDNA}

The 3' RACE protocol requires a population of CDNAs with a known anchor sequence attached to the poly-A tail end of the molecule. It is then possible to PCR amplify the desired CDNA using a degenerate primer based on known peptide sequence (Figure 1.12).

CDNA for 3' RACE was synthesised from pig brain poly-A RNA using Gibco Superscript II reverse trancriptase and the BAT39 synthesis primer (Figure 4.2) as described in Chapter 2 (Section 2.3.4). Since apparently identical cDNA synthesis reactions could produce batches of CDNA of variable quality (Edwards 
et al. 1995), the quality of each batch of CDNA was assessed by PCR amplification of a known low abundance cDNA sequence, trkC (em:M80800). TrkC is a tyrosine protein kinase with a very superficial resemblance to NTE in that it is expressed at low levels, has a similar size (145kDa glycoprotein), and is preferentially expressed in neural tissue (Lamballe et al, 1991). TrkC was selected as the control template as its cDNA was originally isolated from a Clontech pig brain CDNA library, an aliquot of which had been purchased for the NTE cloning project

Primers were designed which covered almost the full length of the published trkC CDNA sequence (Figure 4.3). Two 'forward' primers trk1+ and trk9+, and one 'reverse' primer trk3- were amplified individually and as pairs (trk1+ vs trk3- and trk9+ vs trk3-) to show that the batch of cDNA contained full length copies of low abundance transcripts. A typical result is shown in Figure 4.4.

\subsubsection{Ratification of 3' RACE protocol}

To demonstrate that the $3^{\prime}$ RACE protocol was viable, BAT39-primed CDNA was amplified using the forward primers trk1+, trk5+ and trk9+, and the reverse BAT318 anchor primer (Figure 4.2 and 4.3). Of these primer combinations only trk1+ produced a product band (of about $1.4 \mathrm{~kb}$; Figure 4.5 ). The published CDNA sequence (Figure 4.3) indicates that a 3' RACE product generated from the trk1+ primer should be greater than 1024 bp long (the distance from the trk1+ primer to the end of the published sequence). However, the published trkC cDNA sequence (2.5kbp) contains only 20 bp of 3' untranslated sequence and has no polyadenylation signal (Lamballe et al, 1991). Taking this information into account, it was concluded that the 1.4kbp band observed in Figure 4.5 represented $1 \mathrm{kbp}$ of coding sequence plus a further $0.4 \mathrm{kbp}$ of untranslated sequence from the trkC mRNA. The failure of trk5+ and trk9+ to amplify is unclear. This may have been as a result of insufficient optimisation of PCR conditions, or because the primers were incompatible with 
BAT318. However, since it appeared that the BAT39-primed CDNA was capable of sustaining a 3' RACE reaction, the investigation progressed to perform 3' RACE using degenerate primers derived from pig NTE peptide sequence.

\subsubsection{3' RACE with degenerate primers designed to NTE peptides}

The first series of attempts to clone NTE by 3' RACE used a single degenerate primer designated $\mathrm{F} 10 \mathrm{l}$ which was designed using the pig ADLT peptide sequence (Figure 4.6). The degeneracy of the back translated DNA sequence was reduced from $\times 2048$ down to $\times 32$ by the introduction of inosine bases at the maximally degenerate positions (Ns) in the primer sequence. No inosines were introduced within three bases at the $3^{\prime}$ end of the primer as this was known to adversely affect priming (McPherson et al, 1991).

In combination with BAT318, the F10l primer produced a diffuse but bright band of about 400 to 500 bp in size (Figure 4.7). Further PCR experiments showed that this band was specific to BAT39-primed CDNA, since poly-dT primed CDNA did not produce the band (data not shown). This indicated that a specific reaction with the BAT39 anchor primer was responsible for the product rather than the non-specific priming of BAT318. Titrations of magnesium chloride concentration and annealing temperatures (over the ranges of $1.5-3.0 \mathrm{mM} \mathrm{Mg}^{2+}$ and $55-60^{\circ} \mathrm{C}$ ) failed to produce a more defined product band. Despite its small size (representing approximately 150 amino acids or $16 \mathrm{kDa}$ of protein sequence) the F10I product band was considered significant because poly-dT anchor primers can anneal within an mRNA, on to adenine rich regions of the sequence rather than the poly-A tail, thus yielding a truncated product (Schmidt et al, 1993).

To analyse the 400-500 bp band, it was excised from the agarose gel and the DNA purified by electroelution, before it was cloned into PGEM-T vector (see Section 2.2.2 and 2.2.8 respectively). A total of 18 recombinants were selected 
by blue/white selection and each recombinant colony was propagated in L-Broth $50 \mu \mathrm{g} / \mathrm{ml}$ Ampicillin. Plasmid DNA was purified (Section 2.2.3) and each end of the clone was partially sequenced by Dye Primer cycle sequencing (Section 2.3.2). Of the 18 recombinants, 13 contained inserts produced by F10I/BAT318 primer pair as they contained the F10I and BAT318 DNA sequence at the $3^{\prime}$ and 5 ' ends, respectively. However, when the sequence from the F10I end of the insert was translated, it was found none of the clones possessed predicted peptide sequence matching the ADLT protein sequence known to lie beyond the seven residues used to design the F10I primer (Figures 4.8).

At this stage it was concluded that one of the F10I 3' RACE products which had not been cloned and sequenced might represent an amplified cDNA fragment from NTE, but would have to be separated from the numerous non-specific products also generated by the reaction. To this end a strategy of nested 3' RACE was developed and implemented.

\subsubsection{Nested 3' RACE}

In conventional PCR the two primers each exert a selective pressure on the final product, since for a template to amplify it must possess a binding site for both PCR primers. RACE lacks this double selection and any annealing and extension of the degenerate primer will lead to the formation of a PCR product as all templates possess the reverse anchor primer at the 5' end of the CDNA. This limitation could be in part overcome by nesting pairs of degenerate primers. Products from an initial 3' RACE reaction would be reamplified in a secondary RACE reaction using a different degenerate primer located downstream from the original primer, but the same anchor primer. In context of cloning NTE the major problem was that there was no reliable information as to the relative positions of the various peptide sequences within the NTE protein. Thus it was necessary to test all possible permutations of primer pairing to ensure that the correct arrangement was tested at least once. 
Five more inosine-containing forward (sense) primers F36IS, F36IT, F16I, F13IG and F13/Y were designed aiming to produce oligonucleotides with the lowest degeneracy possible from the peptide sequence available at the time (Figure 4.6). Due to the low annealing temperatures of the degenerate primer pools it was necessary to use a lower annealing temperature of $40^{\circ} \mathrm{C}$ during PCR cycling. Although such a low annealing temperature was liable to cause an increased level of false priming, it was decided that the second round of selection was likely to remove spurious products. Because of the uncertainty regarding the length of the $3^{\prime}$ RACE product (the peptide sequences could be located anywhere in the NTE protein sequence) a very long extension time (7 minutes) was used to ensure even the largest RACE product would be completely extended. The 7 minute extension time was derived using the standard extension rate for Taq polymerase of $1 \mathrm{kbp} /$ minute (Kidd and Ruano, 1995) and the largest plausible template size ( $4.6 \mathrm{kbp}$ coding sequence plus a large $3^{\prime}$ untranslated region).

In the initial experiment BAT39-primed CDNA was amplified with either BAT117 or BAT318 reverse anchor primer paired with each of the new degenerate forward primers (Figure 4.9). Primers BAT117 and BAT318 produced a very similar pattern of bands when paired with NTE degenerate primers, while BAT primers and NTE primers used alone, produced very little product. BAT117 was adopted as the principal RACE anchor primer in the nested 3' RACE reactions since it appeared to give slightly cleaner results relative to the BAT318 and furthermore BAT318 could be used as a nested anchor primer in a possible second or third round of amplification (Figure 4.2).

The first round of 3' RACE produced a bank of eight products for amplification in the second round of RACE. Five templates were derived from degenerate NTE primer vs BAT117. The remaining three were controls comprising; BAT39-primed CDNA amplified with no primers (to show that residual cDNA carried over from the first round of PCR was not responsible for any product formation), BAT39-primed CDNA amplified with BAT117 alone (to control 
for single primer amplification), and a water blank (to control against contamination). These samples were diluted and used as templates in the second round of RACE amplification.

In the second round of nested 3' RACE, all possible permutations of the degenerate primers were tested, even though there were two pairs of primers for which some positional information was available. From Figure 4.6 it can be seen that F13IY was located internally to F13IG and F36IS was internal to F36IT; however, this information was not used to reduce the number of reactions required as it would have complicated the assembling of the nesting reactions and, in any case, use of all primer permutations proved to be a valuable test of the specificity of the second round of PCR.

Each of the six primers (F36IS, F36IT, F16I, F13IG, F13IY and BAT117) were used in PCR reactions as single primers and as a pair with the BAT117 primer on each of the eight templates (in the case of the BAT117 this meant the templates were amplified with no primer at all and with a BAT117 alone). Thus each primer was used in 16 PCR reactions and the full experiment involved a total of 96 different reactions. A typical result and experimental layout for testing of the F36IT primer is described in Figure 4.10.

Several variations on this experiment were tried without success, including nesting the first round products with the $\mathrm{F} 10 \mathrm{O}$ degenerate primer and varying the annealing temperature of the first round RACE amplification. The negative controls (single primer reactions and no template blanks) produced no products indicating that any products that were produced were a result of a RACE reaction between the degenerate primer and the anchor primer. However, the general outcome of these experiments was that first round of RACE produced a large number of products which in the second round of RACE, either failed to reamplify or produced products which were clearly a result of non-specific amplification. 
At this stage in the 3' RACE experiments, the degenerate PCR using primers of inferred orientation which were being performed in parallel with the RACE (Chapter 5) started to show promising results and the 3' RACE experiments were discontinued.

\subsection{Perspectives on Degenerate 3' RACE}

The experiments described in this chapter were intended as a survey of the 3' RACE protocol. They concentrated on testing all the available degenerate primers to determine if any were capable of producing a defined product, either in a single round of amplification, or nested with another degenerate primer. Due to the complexity of assembling all the permutations only a single set of PCR conditions were investigated.

The second stage of the RACE experiments would have concentrated on double checking that the 3' RACE system was adequate for the task of amplifying a large rare transcript using degenerate primers made to trkC protein sequence. This was not attempted in the first series of experiments since it would have represented a task of equal magnitude to cloning NTE.

Once the viability of the system was reaffirmed, all the NTE based degenerate primers were to be extensively optimised with regard to annealing temperature, magnesium concentration, extension time and the effect of using other thermostable DNA polymerases. Additionally, limited nested 3' RACE was planned, using pairs of primers derived from the same peptide sequence (ie. F36IS being internal to F36IT and F13IY being internal to F13IG; see Figure 4.6), so only the appropriate reactions would need to be performed, thus allowing the second round of RACE to be optimised as the first was.

A third approach to the 3' RACE method would have been to Southern blot the products from the first round of RACE. The blot could be probed for hybridisation with each of the degenerate primers end labelled with ${ }^{32} \mathrm{P}$. Any 
band identified by this method could be cloned either by making a library from the 3' RACE products and rescreening it with the degenerate primer, or an antisense primer could be made and used to attempt to PCR amplify the intervening sequence from the cDNA. The advantage of the latter approach would be that the maximum size of the PCR product would be known as it could not be larger than the 3' RACE product it was originally detected in.

A survey of papers which successfully used a 3' RACE cloning approach with degenerate primers, reveals that the products produced by the reported reactions were relatively short and the approximate product size and primer position was known. For example Schmidt et al, (1994) used an N-terminal based inosine containing eight fold degenerate primer to amplify a $1.5 \mathrm{kbp}$ product from plastidial $n-6$ desaturase (a relatively abundant protein from spinach chloroplast). Similarly, Dry and Robinson (1994) a reported cloning $1.65 \mathrm{kbp}$ of the grape berry polyphenol oxidase cDNA (again a relatively abundant protein) with a single primer in which all the degeneracy had been eliminated by substituting inosine in all degenerate positions within the backtranslated sequence.

In both cases there was information as to the approximate size of a product (both laboratories had $\mathrm{N}$-terminal peptide sequence from the intact protein) and furthermore the proteins (and by implication the mRNA) were relatively abundant as only a 100 to 200 fold purification was required to extract the desired protein. The next largest 3' RACE reported was a $\sim 500 \mathrm{bp}$ product amplified from the CDNA of a small GTP binding protein (Rab25) using a 256 fold degenerate primer (Goldenring et al, 1993).

In contrast, NTE is at least an order of magnitude less abundant than the plant proteins (no evidence as to Rab25's abundance was presented in the Goldenring paper). Furthermore it is interesting to look at the final peptide map of NTE in Figure 7.1. Of the four peptide sequences used to design primers for the 3' RACE (Figure 4.6), VILP does not appear in the peptide sequence at all 
(See Figure 7.1 and Appendix 4), ADLT is located $3.9 \mathrm{kbp}$ from the $3^{\prime}$ end of the cDNA sequence, LTNP is $2.0 \mathrm{kbp}$ and DLGL is $1.1 \mathrm{kbp}$ from the $3^{\prime}$ end (see Appendix 4). Thus the successful applications of degenerate 3' RACE were on more abundant templates and/or looking for shorter products, often with some indication of the product length to look for and lower degeneracy peptide sequence. It is possible therefore that degenerate 3' RACE on NTE would have failed regardless of the amount of optimisation, and it was fortunate that it was only one of several approaches to cloning NTE. 


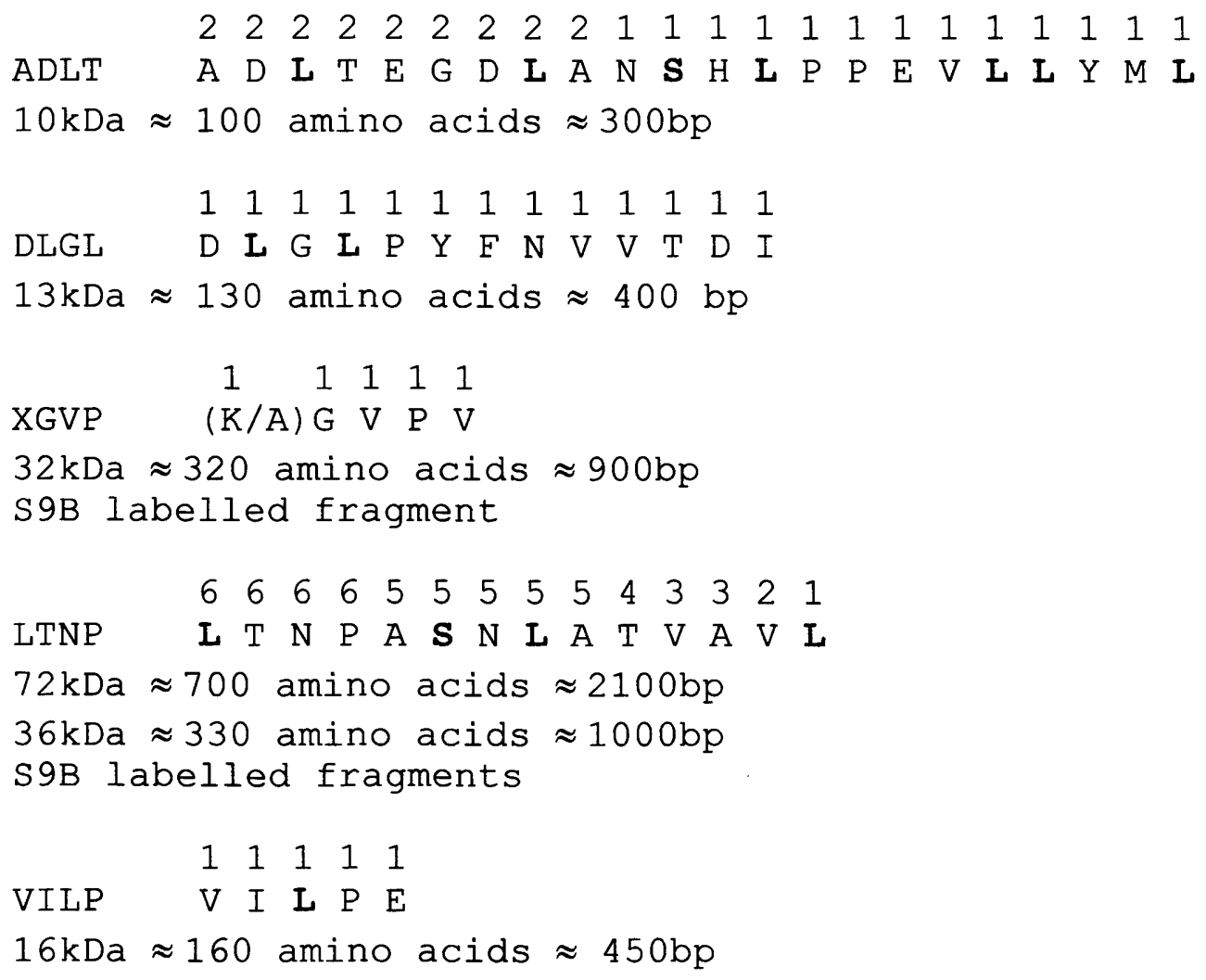

Figure 4.1 Pig NTE peptide sequence available for degenerate primer design.

The number above each residue represents the number of times that residue had been observed in repeated protein sequencing runs. Below the sequence is the size of the V8 digest fragments which yielded the sequences along with the approximate amount of protein and DNA sequence required to encode the peptide. Conversion of molecular weight to DNA sequence assumes the average amino acid weighs 110 Daltons (Da).

Highly degenerate amino acids are highlighted in colour; leucine (green) is 16 fold degenerate, serine (red) is 32 fold. 
Stop Stop

Figure 4.2 Design of anchor primers for reverse transcription of poly-A RNA and 3' RACE.

A novel 20 bp sequence was designed using the program OLIGO for Windows Version 5.0. Primers were designed to have no predicted tendency to form primer dimers either with themselves or the degenerate primers to be used in the cloning attempt and also have maximum priming specificity according to OLIGOs primer annealing model. Stop codons were introduced at the 3' end to discourage anchor primers priming in an open reading frame.

Three oligonucleotides were made using this sequence. One synthesis primer, designated BAT39, had an oligo-dT tail to prime reverse transcription, and two anchor primers were for use as the reverse (antisense) primers in the $3^{\prime}$ RACE reaction. 


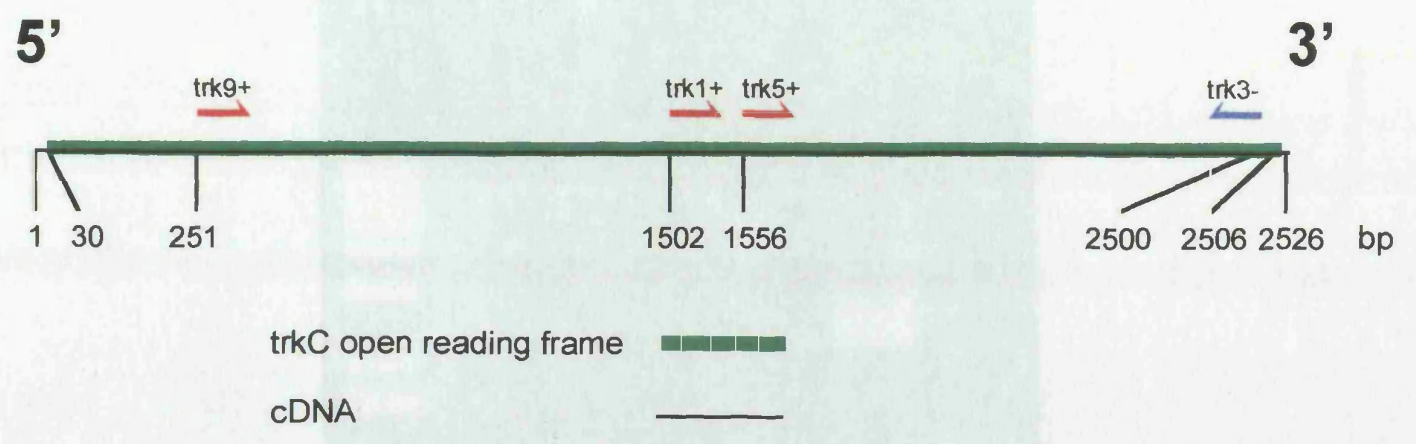

Figure 4.3 Relative positions of trk1+, trk3-, trk5+ and trk9+ primers on pig trkC cDNA.

Trk primers were designed to the published sequence of trkC CDNA (Lamballe et al, 1991. cDNA em:M80800).

\section{Forward primers:-}

$$
5^{\prime}-\cdots>3
$$

trk1+ ACCCTCATCACTGGA

trk5+ CCAGTCATTGAGAACCCC

trk9+ ATCACGGACATCTCAAGG
Reverse primer:-

$$
5^{\prime}-\mathrm{B}^{--.->>}
$$

trk3- GATGTCCCAGGTAGATG

These forward and reverse primers were used as a positive control to test the integrity of the pig brain cDNA BAT39-primed templates used in the PCR and 3' RACE experiments. The products they produced would be of a predictable size (trk9+ vs trk3- $=2249 \mathrm{bp}$, trk1+ vs trk3- $=998 \mathrm{bp}$, trk5+ vs trk3- $=944 \mathrm{bp}$ ) and aid identification of correctly formed product. 


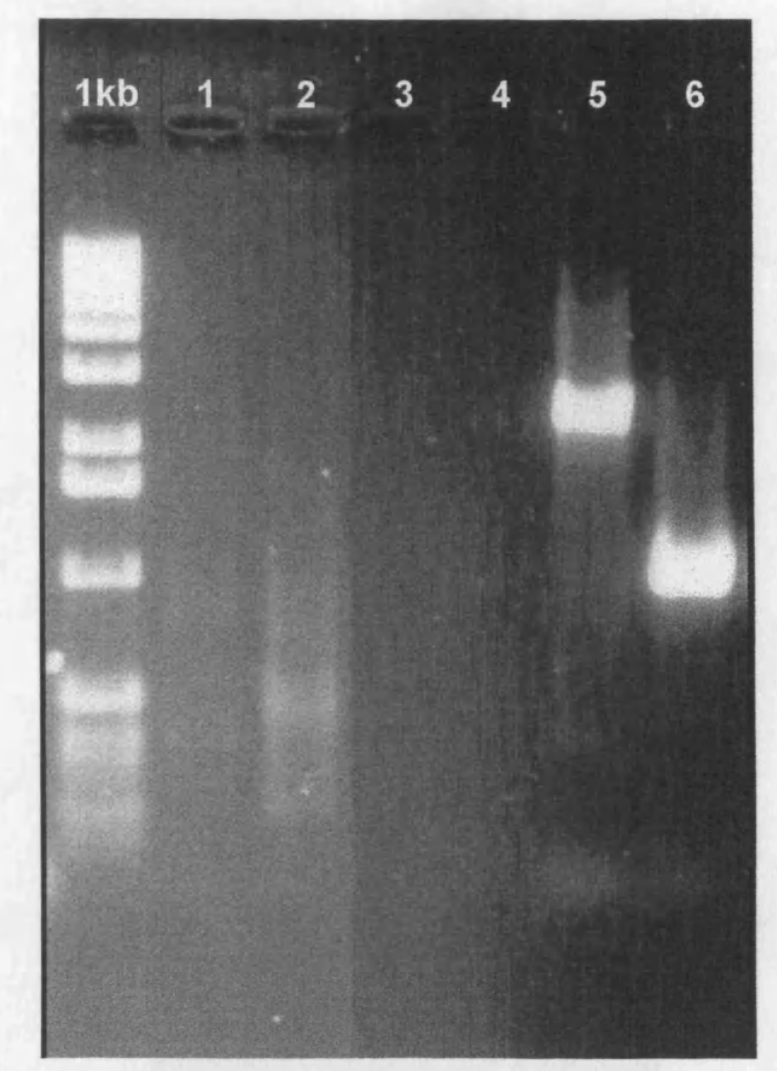

Figure 4.4 Quality control of BAT39-primed cDNA for 3' RACE.

PCR reaction conditions: $\left(94^{\circ} \mathrm{C}, 5 \mathrm{~min},\left[94^{\circ} \mathrm{C}, 1 \mathrm{~min} ; 55^{\circ} \mathrm{C}, 1 \mathrm{~min} ; 72^{\circ} \mathrm{C}\right.\right.$, 2min]x35) $1 \mu \mathrm{M}$ primer, $2 \mathrm{mM} \mathrm{MgCl}, 0.2 \mathrm{mM}$ dNTP, 1 xPCR buffer, $0.5 \mathrm{u}$ Taq2000 polymerase in $20 \mu \mathrm{l}$ reaction volume, $2.5 \mathrm{ng}$ BAT39-primed CDNA).

Lane 5 shows a $2.2 \mathrm{kbp}$ product produced by the trk9+ vs trk3- primer pair, lane 6 shows a $1 \mathrm{kbp}$ product from the trk $1+$ vs trk3- primer pair, lanes 1,2 and 3 show single primers are not responsible for the product bands.

$\begin{array}{ll}\frac{\text { Lane }}{1 \mathrm{~kb}} & \text { Primers } \\ 1 & \text { Gibco 1kbp ladder } \\ 2 & \text { trk1+ alone } \\ 3 & \text { trk9+ alone } \\ 4 & \text { trk3- alone } \\ 5 & \text { no primer blank } \\ 6 & \text { trk9+ vs trk3- } \\ & \text { trk1+ vs trk3- }\end{array}$




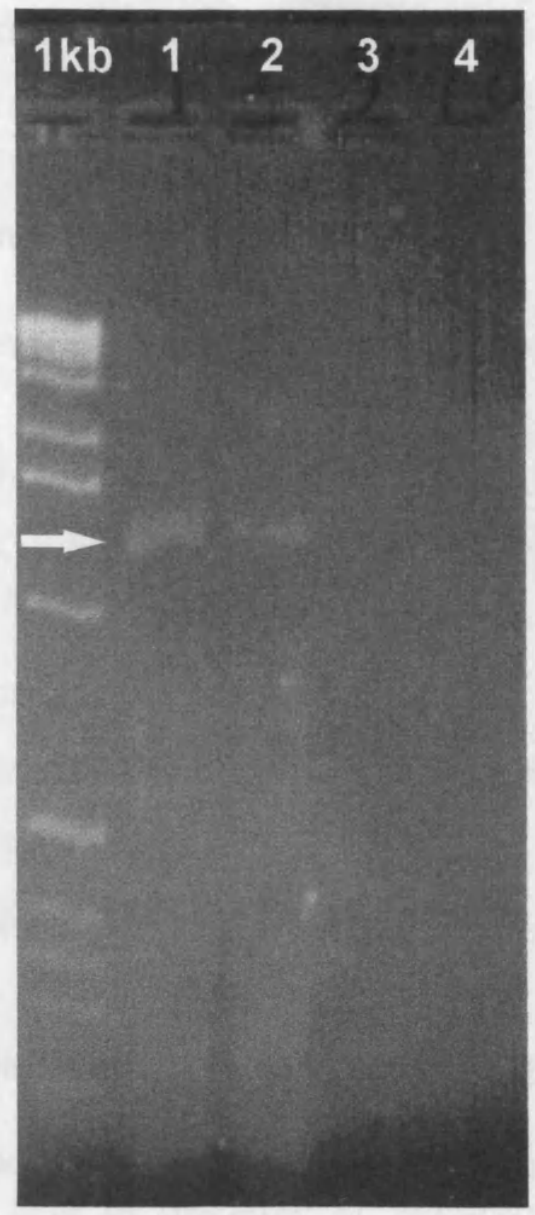

Figure 4.5 Ratification of 3' RACE protocol.

BAT39-primed CDNA was amplified with the forward primers trk9+, trk5+ and trk1+ against the anchor primer BAT318. Only the trk1+/BAT318 primer combination produces any products (shown above). (Conditions $\left(94^{\circ} \mathrm{C}, 5 \mathrm{~min}\right.$, $\left.\left[94^{\circ} \mathrm{C}, 1 \mathrm{~min} ; 55^{\circ} \mathrm{C}, 1 \mathrm{~min} ; 72^{\circ} \mathrm{C} 2 \mathrm{~min}\right] \times 35\right)$, 3ng BAT39-primed cDNA, $1 \mu \mathrm{M}$ each primer, $1.5 \mathrm{mM} \mathrm{MgCl}_{2}, 0.2 \mathrm{mM}$ dNTP, 1xPCR buffer, 1u AmpliTaq polymerase in $30 \mu$ reaction volume).

Lane 1 and 2 are duplicates of the 3' RACE reaction with a major band at about $1.4 \mathrm{kbp}$ (arrowed).

$\begin{array}{ll}\frac{\text { Lane }}{1 \mathrm{~kb}} & \frac{\text { Primers }}{\text { Gibco } 1 \mathrm{kbp} \text { ladder }} \\ 1 & \text { trk1+ vs BAT318 } \\ 2 & \text { trk1+ vs BAT318 } \\ 3 & \text { trk1+ alone } \\ 4 & \text { BAT318 alone }\end{array}$


ADLT (Pig 10kDa) sequence

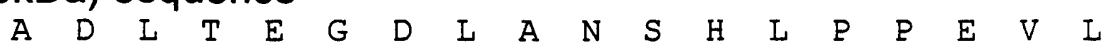

GCNGAYYTNACNGARGGNGAYYTNGCNAAYWSNCAYYTNCCNCCNGARGTNYTN

F10I

GCIGAYYTIACIGARGGNGA

$\times 32 \mathrm{Td}=50-58^{\circ} \mathrm{C}$

DLGL (Pig 13kDa) sequence

$\begin{array}{llllllllllllll}D & L & G & I & P & Y & F & N & V & V & T & D & I\end{array}$

GAYYTNGGNYTNCCNTAYTTYAAYGTNGTNACNGAYATH

F13IG GGIYTICCITAYTTYAA

$x 8 \mathrm{Td}=36-44^{\circ} \mathrm{C}$

F13IY TAYTTYAAYGT IGT IACNGA

$\times 32 \mathrm{Td}=44-52^{\circ} \mathrm{C}$

VILP (Pig 16kDa) sequence

$D E V$ I $L \quad P \quad E$

5 ' GANGTNATHYTNCCNGAR 3 '

F16I

GAIGTIATHYTICCNGA

$\times 24 \mathrm{Td}=38-44^{\circ} \mathrm{C}$

LTNP (Pig 36kDa) sequence

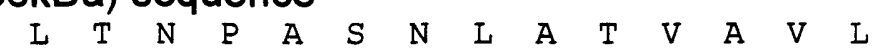

F36IS

E36IT

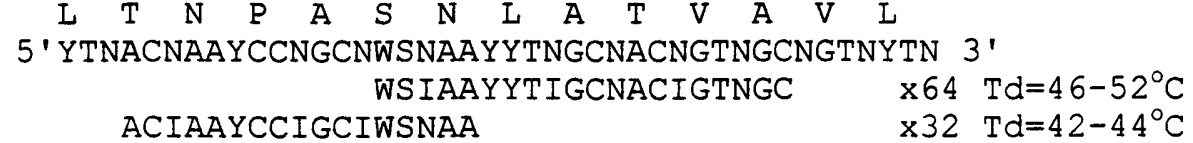

Degeneracy codes: $\mathrm{H}=\mathrm{A}, \mathrm{C}$ or $\mathrm{T}$; $\mathrm{I}=$ inosine; $\mathrm{N}=\mathrm{A}, \mathrm{C}, \mathrm{G}$ or $\mathrm{T}$; $\mathrm{R}=\mathrm{A}$ or $\mathrm{G} ; \mathrm{S}=\mathrm{C}$ or $\mathrm{G} ; \mathrm{W}=\mathrm{A}$ or $\mathrm{T} ; \mathrm{Y}=\mathrm{C}$ or $\mathrm{T}$

Figure 4.6 Design of degenerate primers from NTE peptides for 3' RACE experiment.

Codons shown in bold have been deduced from V8 specificity for peptide bonds on the C-terminal side of glutamic or aspartic acid residues (Drapeau, 1976). The ' $x$ ' values refer to the degeneracy of the primers. The temperature range shown next to the degeneracy is the lowest and highest Td value predicted for the primers in that pool. High and low Td values were calculated using the $2(A+T)+4(G+C)=T d$ formula rule (Kidd and Ruano, 1995) substituting $A$ in all possible positions for the low $\mathrm{Td}$ calculation and substituting $\mathrm{G}$ when calculating the high Td. Inosine was deemed to add 0 to the $\mathrm{Td}$.

Primer positions were selected to make use of the most reliable portions of the peptide sequence and to keep the degeneracy to a minimum. 


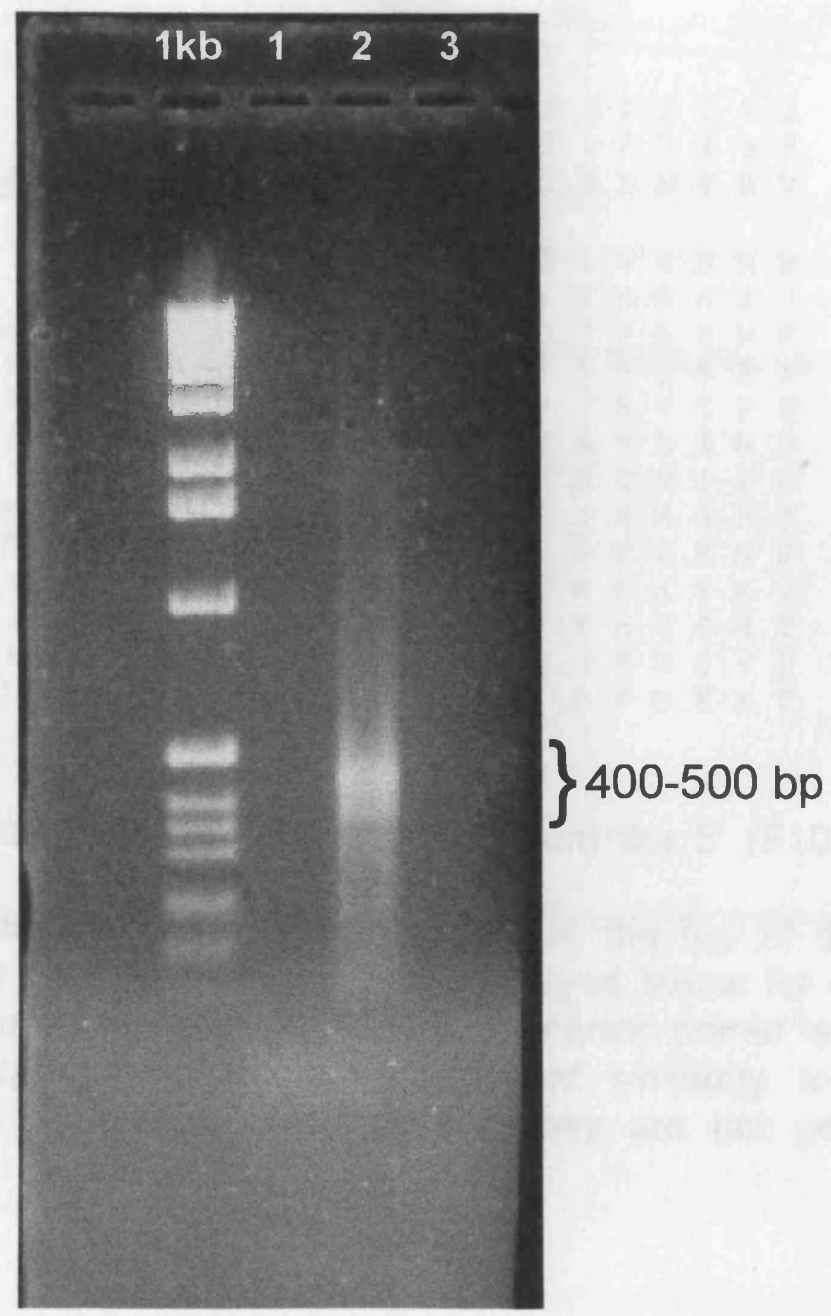

Figure 4.7 3' RACE with the F10I degenerate primer.

BAT39-primed CDNA was PCR amplified with the degenerate primer F10l and RACE anchor primer BAT318. (Conditions $\left(94^{\circ} \mathrm{C}, 5 \mathrm{~min},\left[94^{\circ} \mathrm{C}, 1 \mathrm{~min} ; 55^{\circ} \mathrm{C}\right.\right.$, $\left.\left.1 \mathrm{~min} ; 72^{\circ} \mathrm{C}, 3 \mathrm{~min}\right] \times 35\right)$, 3ng BAT39 cDNA $1.7 \mu \mathrm{M}$ F10I, $0.1 \mu \mathrm{M}$ BAT318, $1.5 \mathrm{mM}$ $\mathrm{MgCl}_{2}, 0.2 \mathrm{mM}$ dNTP, 1xPCR buffer, 1u AmpliTaq polymerase in $30 \mu$ reaction volume).

The reaction produced a broad band of 400 to $500 \mathrm{bp}$. This band though relatively small was interesting as it was known that the RACE protocol could produce truncated products if the BAT39 annealed within the MRNA, as opposed to the poly-A tail (Schmidt et al, 1993).

$\begin{array}{ll}\frac{\text { Lane }}{1 \mathrm{~kb}} & \frac{\text { Primers }}{\text { Gibco } 1 \mathrm{kbp} \mathrm{ladder}} \\ 1 & \text { BAT318 alone } \\ 2 & \text { F10l vs BAT318 } \\ 3 & \text { F10l alone }\end{array}$


Residue number

$\begin{array}{llllllllllllllllll}1 & 1 & 1 & 1 & 1 & 1 & 1 & 1 & 1 & 1 & 2 & 2 & 2 & 2 & 2 & 2 & 2\end{array}$

$\begin{array}{llllllllllllllllllllllllll}1 & 2 & 3 & 4 & 5 & 6 & 7 & 8 & 9 & 0 & 1 & 2 & 3 & 4 & 5 & 6 & 7 & 8 & 9 & 0 & 1 & 2 & 3 & 4 & 5 & 6\end{array}$

A D I T E G D I A N S H I P P E V I I Y M I N V R V

Clone ID

r3-12 A D L T E G D * Q V K M Q F G S S H C G S V E K N P r2-13A A D I T E G D N F K * R V L Y N * F K F V K G S R V r1-17A A D L T E G D r3-09A A D L T E G E E D * T K P N L E X S

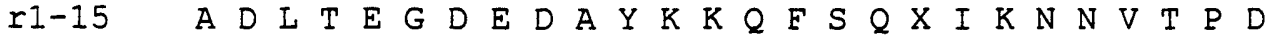
11-11 A D L T E G D r3-05A A D L T E G D E D A Y K X $Q$ Q X S $Q$ I2-06A A D L T E G E E D * T K P X S X K S * H I H X S H r2-04A A D L T E G D $Q Q V$ X M Q F G S S H C X S V E K N P I2-14A A D L T E G D K C X S S H D L T I E R K X E X R R V r3-01A A D L T E G E E D * P T P X X E K S $\mathrm{T}$ Y I H Q S H T

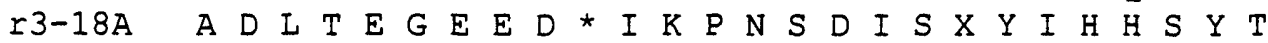
I1-16A A D L T E G E R X X X X X G R A R

Figure 4.8 Translations of the DNA sequences from the $5^{\prime}$ (F10I) end of $3^{\prime}$ RACE products.

The NTE sequence ADLT is shown in bold at the top of the table for comparison with the $13 \mathrm{~F} 10 \mathrm{l}$ derived sequences arrayed below for comparison. The peptide sequence used to design the $\mathrm{F} 10 \mathrm{l}$ degenerate primer is underlined. None of the F10l sequences show any significant similarity to the ADLT sequence beyond the $7^{\text {th }}$ residue demonstrating they are not genuine NTE clones. 


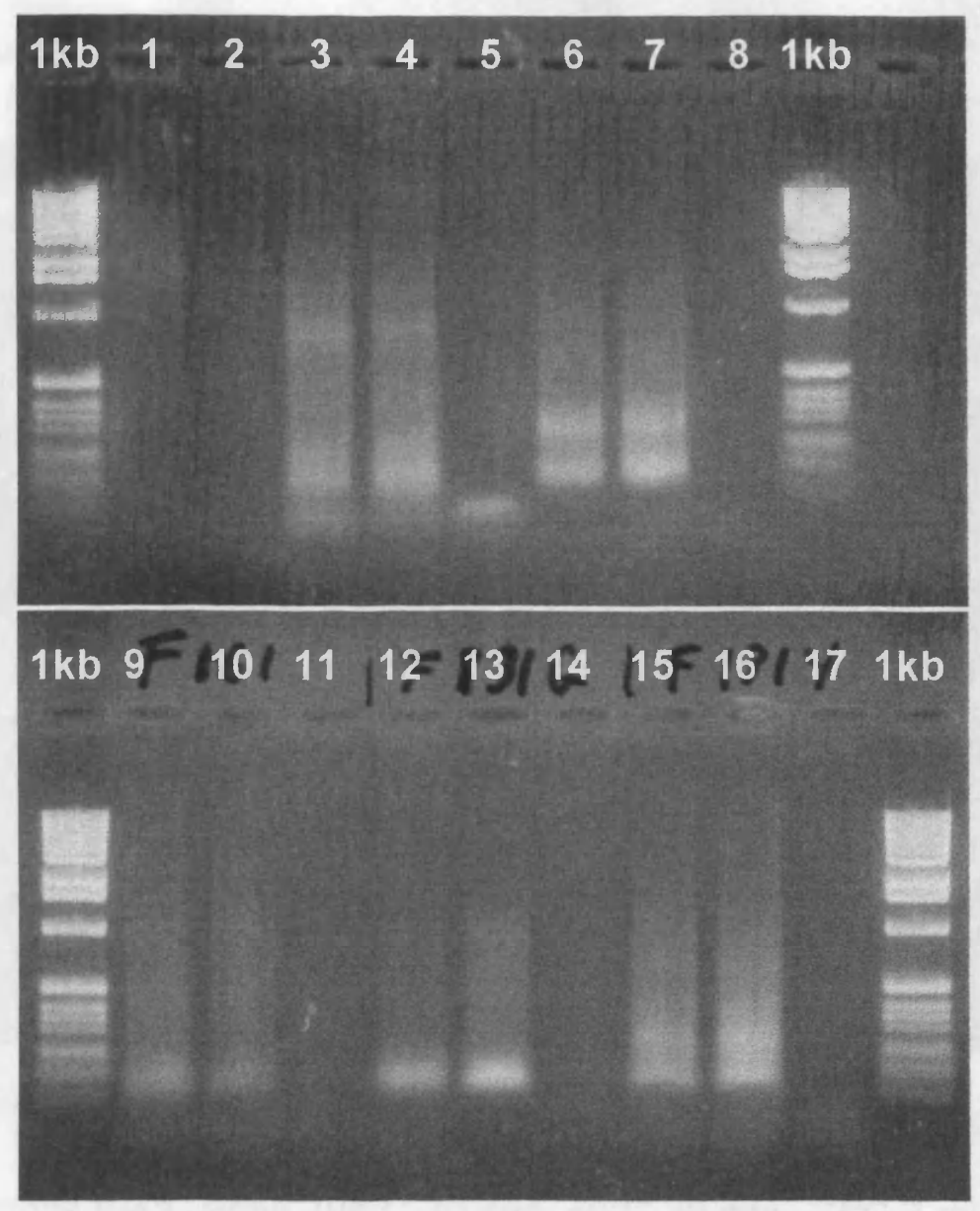

Figure 4.9 PCR amplification of BAT39-primed CDNA with degenerate primers F36IS, F36IT, F16I, F13IG and F13IY paired with reverse primers BAT117 and BAT318.

Amplification conditions $\left(94^{\circ} \mathrm{C}, 5 \mathrm{~min},\left[94^{\circ} \mathrm{C}, 30 \mathrm{sec} ; 40^{\circ} \mathrm{C}, 30 \mathrm{sec} ; 72^{\circ} \mathrm{C}, 7\right.\right.$ min]x35), 3ng BAT39 cDNA, $1.7 \mu \mathrm{M}$ Degenerate primer, $0.1 \mu \mathrm{M}$ BAT318 or BAT117, $1.5 \mathrm{mM} \mathrm{MgCl}_{2}, 0.2 \mathrm{mM}$ dNTP, 1xPCR buffer, 1u AmpliTaq polymerase in $30 \mu$ reaction volume).

\begin{tabular}{|c|c|c|c|}
\hline Lane & Primers & Lane & Primers \\
\hline$\overline{1}$ & $\overline{\text { BAT318 alone }}$ & $\overline{9}$ & F16I vs BAT318 \\
\hline 2 & BAT117 alone & 10 & F16I vs BAT117 \\
\hline 3 & F36IS vs BAT318 & 11 & F16l alone \\
\hline 4 & F36IS vs BAT117 & 12 & F13IG vs BAT318 \\
\hline 5 & F36IS alone & 13 & F13IG vs BAT 117 \\
\hline 6 & F36IT vs BAT318 & 14 & F13IG alone \\
\hline 7 & F36IT vs BAT117 & 15 & F13IY vs BAT318 \\
\hline 8 & F36IT alone & 16 & F13IY vs BAT117 \\
\hline $1 \mathrm{~kb}$ & Gibco $1 \mathrm{kbp}$ ladder & 17 & F13IY alone \\
\hline
\end{tabular}




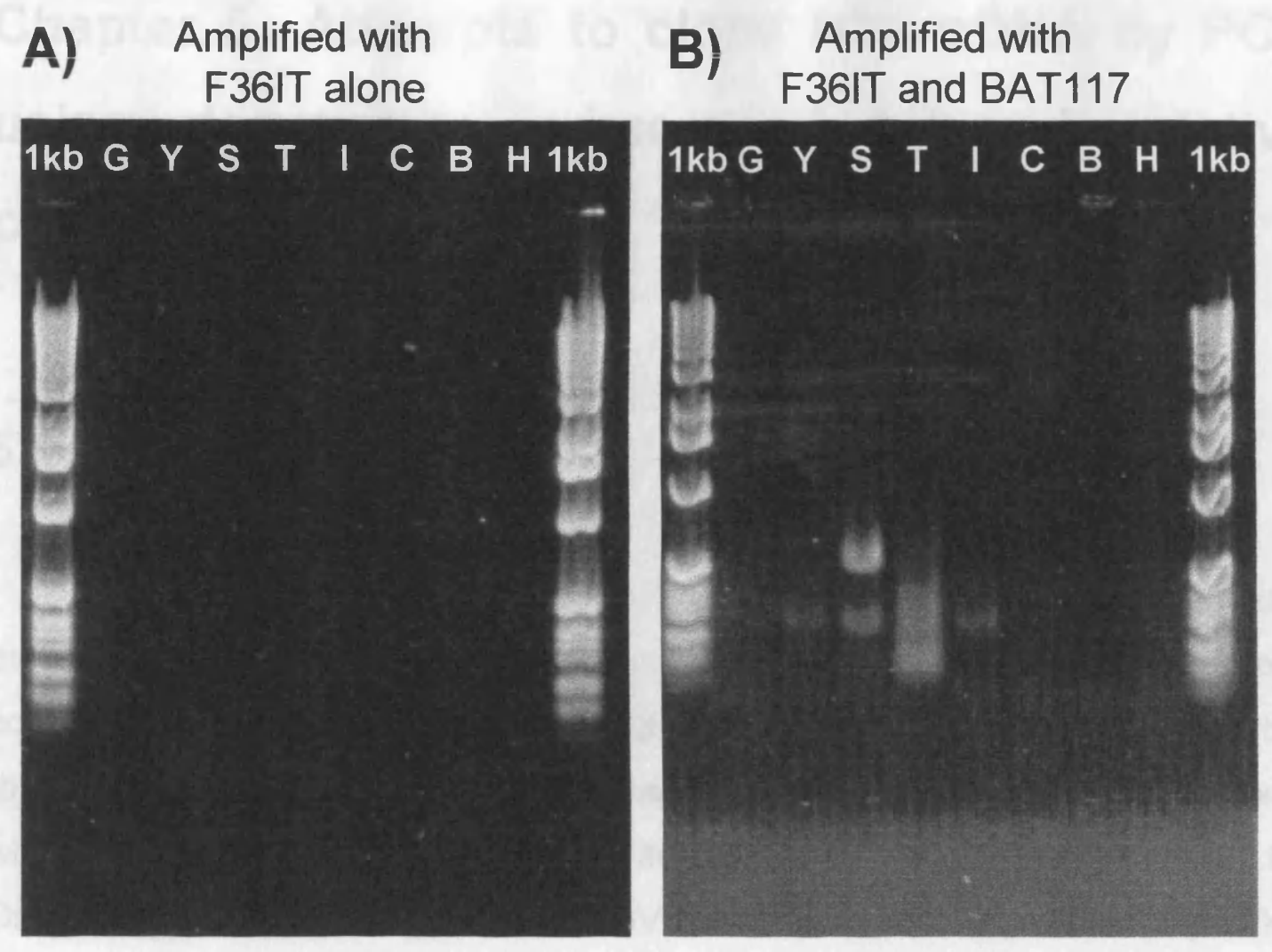

Figure 4.10 Typical example of 2nd round nested 3' RACE with degenerate primers.

Conditions $\left(94^{\circ} \mathrm{C} 5 \mathrm{~min},\left[94^{\circ} \mathrm{C} 30 \mathrm{sec}, 40^{\circ} \mathrm{C} 30 \mathrm{sec}, 72^{\circ} \mathrm{C} 7 \mathrm{~min}\right] \times 35\right) \quad 1.7 \mu \mathrm{M}$ F36IT, $0.1 \mu \mathrm{M}$ BAT117, $1.5 \mathrm{mM} \mathrm{MgCl}_{2}, 0.2 \mathrm{mM}$ dNTP, $1 \times$ PCR buffer, $1 \mathrm{u}$ AmpliTaq polymerase in $30 \mu \mathrm{l}$ reaction volume, $5 \mu \mathrm{l} 1: 10000$ diluted template from 1 st round amplification).

Template codes:

First round PCR reaction products amplified with BAT117 paired with;

G, F13IG; Y, F13IY; S, F36IS; T, F36IT; I, F16I.

Negative control templates were; C, BAT39 cDNA diluted 1:10000; B, first round PCR amplified by BAT117 alone; $H$, water blank.

Gel A shows each template does not amplify in the presence of the F36IT alone. The BAT117 primer also shows no amplification on these templates (Data not shown).

Gel B shows the products produced by the F36IT/BAT117 primer pair. The products in lane $S$ were potentially interesting, as a specific product of about $500 \mathrm{bp}$ was produced. However the template for that reaction was made using F36IS/BAT117 primer pair and since F36IS is located internally to F36IT (Figure 4.6), the $500 \mathrm{bp}$ product must be the result of non-specific hybridisation. Lane $T$ shows the non-specific amplification resulting from F36IT reamplifying F36IT derived template. 


\section{Chapter 5 Attempts to clone NTE cDNA by PCR using degenerate primers of inferred relative position}

\subsection{Introduction}

Chapter 3 described experiments in which S9B-labelled purified NTE, was digested with V8 protease before it was refractionated on an avidin-Sepharose column (Section 3.2.2). This allowed the separation of fragments bearing the (biotinylated) active site, from the complex mixture of NTE fragments. Fragments which passed through the column were deemed to be unbiotinylated (Figure 3.8). Only two sequences, LTNP and XGVP were found in the avidin repurified fraction and they were associated with protein fragments of 16 to $72 \mathrm{kDa}$ in size (Figure 3.7). The simplest organisation of the two peptides (ie, the one which required the minimum number of V8-mediated bond cleavages) resulted in the map depicted in Figure 5.1. In terms of this scheme, a PCR using forward primers designed to the LTNP sequence and reverse primers designed using XGVP sequence should produce a PCR product of approximately $100 \mathrm{bp}(\sim 4 \mathrm{kDa}$ of peptide sequence). As discussed in Chapter 1 (Section 1.3.1) this is a useful observation as gave some clue as to what primers to use and what product size to expect.

\subsection{Results and discussion}

\subsubsection{Production and isolation of PCR-generated clone ORF1}

Two forward primers were designed to the LTNP peptide sequence and two reverse primers were designed to the XGVP peptide sequence using inosine to reduce the overall degeneracy of the primers; these primers were designated 
F36IT, F36IS, R32IA and R32IK (Figure 5.2). All permutations of forward and reverse primers were tested in PCR reactions with oligo-dT primed pig brain cDNA at magnesium chloride concentrations of $1.5 \mathrm{mM}$ to $4 \mathrm{mM}$; it was found that certain primer pairs produced product bands of about 170, 300 and 400 bp with $3 \mathrm{mM}$ magnesium chloride (Figure 5.3).

The 170 bp product band, which was the closest to the predicted size, was excised from the agarose gel and reamplified, using the same reaction conditions. This produced sufficient DNA to clone into the PGEM-T vector system (Section 2.2.8). Eighteen recombinants were picked by blue/white selection, then propagated in L-Broth containing $50 \mu \mathrm{g} / \mathrm{ml}$ Ampicillin. Plasmid DNA was isolated and the inserts sequenced on both strands by Dye Primer cycle sequencing (see Section 2.3.2).

Out of the eighteen transformants, six did not contain a 170 bp insert and were discarded, four clones contained an identical sequence which was dubbed ORF1. A further two clones contained a different sequence named ORF2. The remaining six clones each contained different sequences, each one with a stop codon in the LTNP primers reading frame, thus they were eliminated as candidate clones possessing NTE cDNA sequence. The ORF1 and ORF2 sequences were translated in all 3 reading frames defining the F36IS sequence as the 5' end (Figure 5.4). The ORF2 sequence was dismissed as a candidate since, when translated, the F36IS and F32IK peptide sequences occurred in different reading frames. This frameshift was unlikely to be a result of a Taq polymerase-mediated mutation since two identical ORF2 clones were observed and, furthermore, Taq polymerase generates relatively few frameshift mutations (Tindall and Kunkel, 1988); thus investigation of ORF2 was suspended in favour of the ORF1 clone.

The ORF1 sequence was thought to be potentially interesting since both known NTE peptide sequences were in the same reading frame and were linked by ORF1's only open reading frame. The mismatch between the predicted 
residues at the C-terminal of LTNP (VL) and the observed ones (TT), was thought to be a result of errors in protein sequencing. Both serine and threonine residues are difficult to detect by Edman sequencing as the anilinothiazolinoneamino acids (AZT-amino acids) cleaved from the peptide chain undergo a $\beta$ elimination process resulting in derivatised amino acids. When yields of amino acids are low (as was the case in the sequencing of NTE) the derivatives are hard to detect: serine can be detected as dehydroalanine; threonine co-elutes with proline, methionine and valine derivatives, effectively concealing it from detection (K. Lilley, Personal communication). The mismatch of the first residue ( $S$ vs R) was a result of the degenerate codon used in that position (WSN) as it codes for both serine and arginine. Extensive database searches were carried out using the BLAST, FASTA and BLITZ programs on both the DNA and deduced peptide sequence of ORF1, but produced no significant matches in either EMBL or SWISSPROT.

It was noticed that one of the predictions made by the putative peptide map (Figure 5.1) placed the LTNP sequence 20 to $27 \mathrm{kDa}$ (180-250 amino acids) from the catalytic serine. Interestingly, the $\mathrm{N}$-terminus of the acetylcholinesterase (AChE) family of serine esterases also lies approximately 200 residues from the catalytic serine (Krejci et al, 1991). This prompted an attempt to align the deduced ORF1 peptide sequence with the $\mathrm{N}$-terminal region of the serine esterase alignment. (Figure 5.5). The degree of similarity revealed by the alignment between ORF1 and a portion of a known serine esterase family was thought sufficient evidence to justify cloning the flanking DNA sequence and determine if similarity really extended through and beyond the primer sequence. 


\subsubsection{Attempts to clone the ORF1 template}

Various approaches were taken to clone the template which produced ORF1.

1) CDNA library screening

Hybridisation screening of 1 million plaques from a Clontech pig brain cDNA library using ${ }^{32} \mathrm{P}$ labelled ORF1 probe, yielded no genuine positive clones (Section 2.4.1).

\section{2) 3'RACE}

Non-degenerate forward primers were designed using ORF1 DNA sequence lying between the degenerate primers (two of these primers are shown in Figure 5.6). The primers were used in an unsuccessful attempt at 3' RACE on BAT39-primed CDNA which failed to produce any specific products despite optimisation and the use of nested primers.

\section{3) 'hemi-degenerate' PCR}

Previous work by the group (Section 1.1.5) had produced a limited amount of heterogeneous sequence, flanking the catalytic serine (see Figure 1.7; Glynn et al, 1993). In an attempt to elucidate the genuine sequence, SWISSPROT was BLITZ searched using the acetylcholinesterase active site sequence (VTLFGESAG). Twentyone different active sites were observed and the sequences combined into a catalytic site motif which was compared with this heterogeneous NTE sequence (Figure 5.7). The tentative consensus sequence residues was used to design two degenerate primers, denoted F-SECl and RSECI. These primers were hardly used during the NTE cloning project, due to the highly speculative nature of their generation. However the R-SECl (the reverse primer) was tested with the nondegenerate ORF1 based forward primers (Figure 5.6) but produced no product (data not shown). 
4) Inverse PCR

Inverse PCR is a method which allows the sequence flanking a short section of known DNA sequence to be specifically amplified (Ochman et al, 1990). The technique requires genomic DNA digested with a restriction enzyme to produce a population of DNA fragments each terminating in compatible sticky ends. The digested DNA is religated at a low DNA concentration $(<2 \mu \mathrm{g} / \mathrm{ml})$. This promotes intramolecular ligation over intermolecular ligation, thus the fragments self ligate to form circles rather than randomly reassociate with other fragments. PCR with non-degenerate primers pointing outwards from the known sequence amplifies the bulk of the circle resulting in novel sequence flanked by known (ORF1) sequence (Figure 5.8).

High molecular weight pig genomic DNA was prepared from frozen pig brain tissue (see Section 2.2.6 and 2.3.4). Two circularised genomic DNA (cgDNA) libraries were prepared using Taql- and Mbol-digested DNA. Restriction enzymes were selected on the basis that they produced 4bp sticky ends and that they did not cut the known ORF1 sequence. Two cgDNA libraries were constructed to guard against an enzyme that produced an ORF1 bearing fragments which were too large ( $>3 \mathrm{kbp}$ ) to easily amplify from a complex mix, or too small (<200-300 bp) to readily circularise (Silver, 1991).

The circularised template was amplified with the ORFA vs ORRA as a positive control for the presence of the ORF1 sequence, as well as the inverse primers ORFD vs ORRA (Figures 5.6 and 5.9). Despite the very poor quality of the single primer controls and the ORFA vs ORRA positive control, an $800 \mathrm{bp}$ band was considered worth pursuing and the gel was Southern blotted (see Section 2.4.2). When the blot was probed with ${ }^{32} \mathrm{P}$-labelled ORF1 (see Section 2.4.3 and 2.4.4) a hybridisation signal was detected around the 800 bp band, so the entire PCR reaction which produced the band was cloned into PCR-script vector (Section 2.2.9). 
Twenty recombinant colonies were selected on the basis of Blue/White selection and the ORF1 inserts were selected for by a diagnostic PCR between the ORFE vs vector primer. This produced a PCR product of predicted size (data not shown). On the basis of this experiment six colonies were selected (clones 996A 1, 3, 5, 18, 19 and 20) and the inserts sequenced by Dye Primer cycle sequencing (Section 2.3.2). All six clones were found to posses a $100 \%$ DNA match with the ORF1 sequence located between the degenerate primers, but only the first 9 bases of the primer were homologous to the ORF1 sequence; beyond that the known NTE peptide sequences and the translated sequence of the genomic clone diverged, proving conclusively that ORF1 did not represent a part of the NTE CDNA sequence and was merely the product of non-specific amplification (Figure 5.10).

\subsection{Retrospective view on the relative positions of LTNP, XGVP and the catalytic serine in NTE}

Now that NTE has been cloned and sequenced (Figure 7.1) it is possible to construct a more realistic peptide map surrounding the S9B-labelled serine residue (Figure 5.11). Most significantly, LTNP is 228 residues ( 25kDa) upstream of XGVP, rather than the $4 \mathrm{kDa}$ predicted by the map in Figure 5.1. Part of the reason for this margin of error was the mistaken impression that the $16 \mathrm{kDa}$ XGVP fragment was not biotinylated, when, in fact, it was, but its presence in the $V 8$ avidin eluate fraction was masked by the avidin monomer (Figure 3.8). A certain percentage of the $16 \mathrm{kDa}$ XGVP fragment was always detected in the avidin flowthrough fraction because, under the experimental conditions used $(0.15 \%$ SDS), avidin-biotin binding is not totally efficient. In the light of the complete sequence data (Figure 7.1) it is clear that a PCR reaction between primers corresponding to LTNP and XGVP should yield a product of about 680bp. However, the largest discernible product do the degenerate PCR was only about $400 \mathrm{bp}$ (Figure 5.3), thus it is probable that this approach would have still failed. 
More importantly, since the LTNP is $25 \mathrm{kDa}$ upstream of XGVP and the $36 \mathrm{kDa}$ LTNP fragment contains the S9B labelling site, then the catalytic serine must lie within the $11 \mathrm{kDa}$ (100 residues) downstream of the XGVP sequence. 


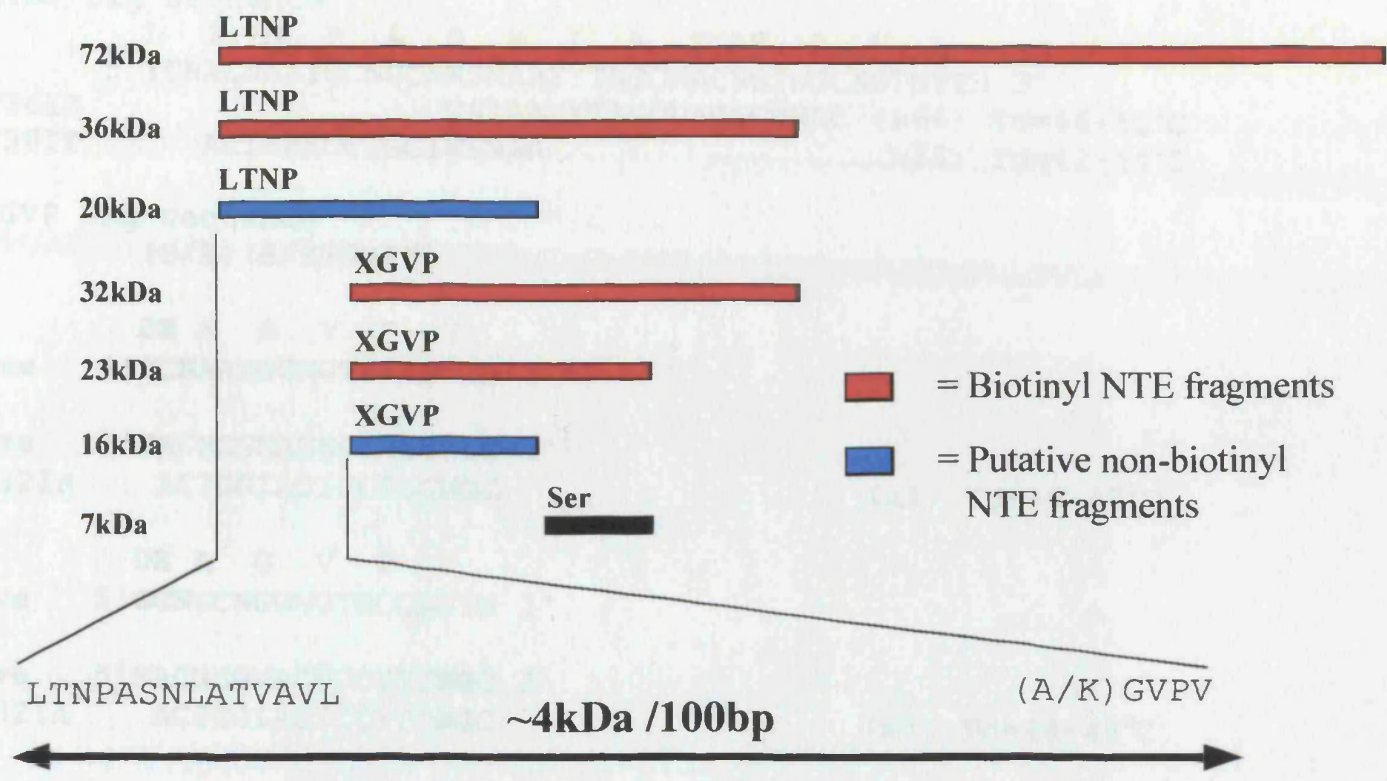

Figure 5.1 Putative peptide map of the serine esterase domain of NTE.

Originally the map only consisted of the $32 \mathrm{kDa}$ XGVP sequence and the 36 and $72 \mathrm{kDa}$ LTNP peptide sequences; later sequencing work provided the other biotinyl and nonbiotinyl sequences which expanded and apparently confirmed the map.

The LTNP peptide sequence was eventually found to be associated with protein fragments of 72,36 and $20 \mathrm{kDa}$. The 72 and $36 \mathrm{kDa}$ fragments were biotinylated by S9B, the $20 \mathrm{kDa}$ was possibly unbiotinylated. Similarly XGVP sequence was associated with two biotinylated fragments ( 32 and $23 \mathrm{kDa})$ plus one $16 \mathrm{kDa}$ protein fragment which also appeared not to be biotinylated.

This information was used to construct the map shown above and infer the position of the catalytic serine (marked Ser) to between 20 to $27 \mathrm{kDa}(180-250$ amino acids) from the LTNP peptide sequence. The map also predicted a $4 \mathrm{kDa}$ distance between the LTNP and XGVP sequences. This information was used to estimate a range of plausible product sizes from a PCR reaction using degenerate primers designed to the peptide sequence. 


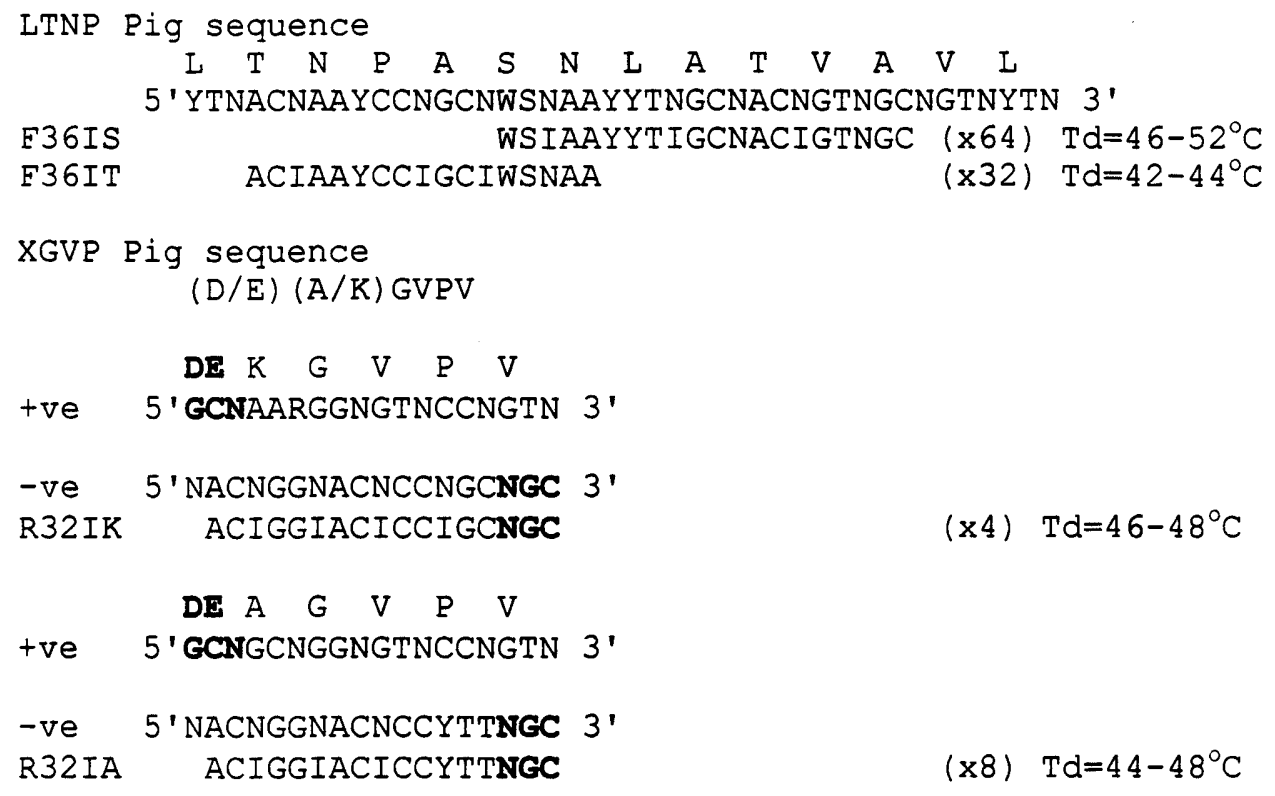

Figure 5.2 Design of degenerate primers to the LTNP and XGVP peptide sequences.

The LTNP sequence posed no unusual problems in selecting PCR primers (aside from the degeneracy of the sequence).

The XGVP peptide sequence posed two problems when designing reverse degenerate primers.

First, the Edman sequencing was unable to determine if the first residue in the sequence was an alanine or a lysine so two primers were designed, one assuming the 1st position was an alanine (R32IA) the other assuming it was a lysine (R32IK).

Secondly the sequence was too short to produce PCR primers with a reasonable $\mathrm{Td}$ value so the residue preceding the $\mathrm{N}$-terminal was inferred from the specificity of V8 protease for glutamate and aspartate which combined to from the codon GCN (shown in bold) increasing the annealing temperature of the primers by $8-10^{\circ} \mathrm{C}$. 


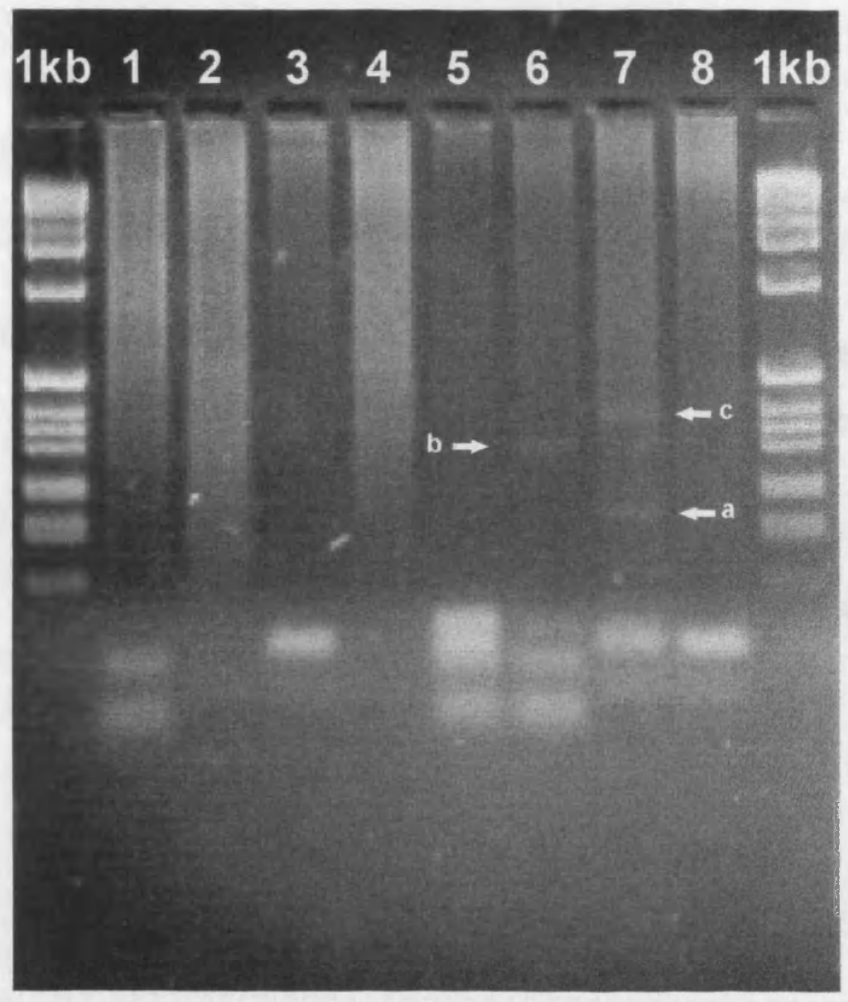

Figure 5.3 PCR amplification of oligo-dT primed pig brain CDNA using primers of putatively known position.

All combinations of forward vs reverse primers were tested (Conditions $\left(94^{\circ} \mathrm{C} 5 \mathrm{~min},\left[94^{\circ} \mathrm{C} 1 \mathrm{~min} ; 55^{\circ} \mathrm{C} 1 \mathrm{~min} ; 72^{\circ} \mathrm{C} 2 \mathrm{~min}\right] \times 35\right), 3 \mathrm{ng}$ pig brain oligo-d $\mathrm{T}$ primed cDNA $1 \mu \mathrm{M}$ primer, $3 \mathrm{mM} \mathrm{MgCl}_{2}, 0.2 \mathrm{mM}$ dNTP, 1 xPCR buffer, $0.5 \mathrm{u}$ Taq2000 polymerase in $20 \mu \mathrm{l}$ reaction volume).

Three faint bands (arrowed a, b and c) were evident in lanes 6 and 7 , of sizes a $\sim 170 \mathrm{bp}, \quad b \sim 300 \mathrm{bp}, \mathrm{c} \sim 400 \mathrm{bp}$.

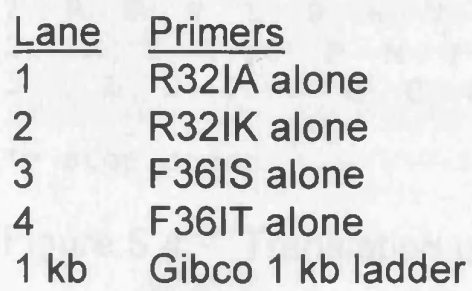

\begin{tabular}{|c|c|}
\hline Lane & Primers \\
\hline 5 & F36IS vs R32IA \\
\hline 6 & F36IT vs R32IA \\
\hline 7 & F36IS vs R32IK \\
\hline 8 & F36IT vs R32IK \\
\hline
\end{tabular}




\section{ORF1}

LTNP (Pig $36 \mathrm{kDa}$ ) sequence residues $6-14$

$\begin{array}{llllllllll}S & N & L & A & T & V & A & V & L\end{array}$

5' AGGAACCTGGCGACGGTGGCCACTACCACAGGTCAGGAAATGGGACTGAATATTGGGCCT

$\begin{array}{lllllllllllllllllllllllll}1 & \mathbf{R} & \mathbf{N} & \mathbf{I} & \mathbf{A} & \mathbf{T} & \mathbf{V} & \mathbf{A} & \mathrm{T} & \mathrm{T} & \mathrm{T} & G & \boldsymbol{Q} & \mathrm{E} & \mathrm{M} & \mathrm{G} & \mathrm{L} & \mathrm{N} & \mathrm{I} & G & \mathrm{P}\end{array}$

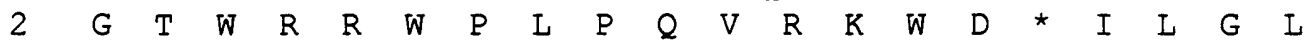

$\begin{array}{lllllllllllllllllllllll}3 & & E & P & G & D & G & G & H & Y & H & R & S & G & N & G & T & E & Y & W & A & F\end{array}$

TCCATGTGTTCTGTATTGCTCATTATCTATTTAAAGCTTAGAAGTGTTTTTCTTATTTTC

$\begin{array}{lllllllllllllllllllll}1 & S & M & C & S & V & L & L & I & I & Y & L & K & L & R & S & V & F & L & I & F\end{array}$

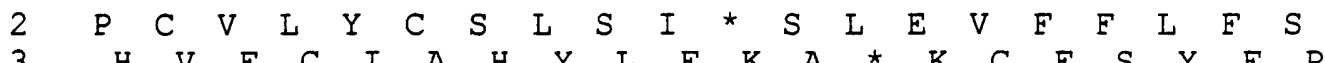

$$
\text { XGVP (Pig } 32 \mathrm{kDa} \text { ) sequence }
$$

$D \quad K \quad G \quad V \quad P \quad V$

CTGCAGGTGGTTATCACTAGACTAAAACTGGACAAGGGCGTCCCCGT 3 '

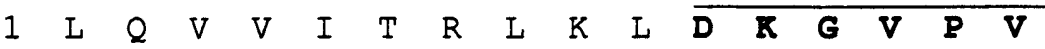

$\begin{array}{lllllllllllllllll}2 & C & R & W & L & S & L & D & * & N & W & T & R & A & S & P\end{array}$

$\begin{array}{llllllllllllllllll}3 & A & G & G & Y & H & * & T & K & T & G & Q & G & R & P & R\end{array}$

\section{ORF2}

LTNP (Pig $36 \mathrm{kDa}$ ) sequence residues 6-14

$S \quad N \quad L \quad A \quad T \quad V \quad A \quad V \quad$ L

5 ' AGGAACCTGGCGACGGTGGCCCATCTGGTAAATGAAGATCCCCTCCAAAAGTCATTATTT

$\begin{array}{llllllllllllllllllllllll}1 & \mathbf{R} & \mathbf{N} & \mathbf{I} & \mathbf{A} & \mathbf{T} & \mathbf{V} & \mathbf{A} & \mathrm{H} & \mathrm{L} & \mathrm{V} & \mathrm{N} & \mathrm{E} & \mathrm{D} & \mathrm{P} & \mathrm{L} & \mathrm{Q} & \mathrm{K} & \mathrm{S} & \mathrm{L} & \mathrm{F}\end{array}$

$\begin{array}{lllllllllllllllllllll}2 & G & T & W & R & R & W & P & I & W & * & M & K & I & P & S & K & S & H & Y & I\end{array}$

$\begin{array}{llllllllllllllllllllll}3 & E & P & G & D & G & G & P & S & G & K & * & R & S & P & P & K & V & I & I & C\end{array}$

GTAGATTATATGATCACATACAGCTTTGGGTCTGGAACAGTCTTTGCCTTTCATTCTCAG

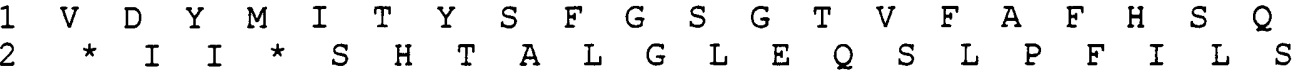

$\begin{array}{lllllllllllllllllllll}3 & R & L & Y & D & H & I & Q & L & W & V & W & N & S & L & C & L & S & F & S & A\end{array}$

XGVP (Pig $32 \mathrm{kDa}$ ) sequence

CGCTTCCACCTGTCCCATGTTCCTGGACAAAGGCGTCCCCGT $3^{\prime}$

$\begin{array}{lllllllllllllll}1 & R & F & H & L & S & H & V & P & G & Q & R & R & P & R\end{array}$

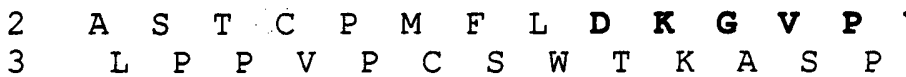

* = stop codon

Figure 5.4 Translation of ORF1 and ORF2 sequence in three reading frames. 
Figure 5.5 Alignment ORF1 with the $\mathrm{N}$-termini of multiple serine esterase domains.

Residues identical to ORF1 are highlighted in red, those similar to ORF1 are marked in green. Similarity was defined as the amino acid pair having a score of 0 or greater on the BLOSUM62 protein comparison matrix (Henikoff and Henikoff, 1992).

The original alignment (Krejci et al, 1991) did not contain the sequence for neuroligins 1,2 or 3 , or gliotactin, these were added to the alignment later. The addition of gliotactin was considered particularly auspicious, since it is an inactive serine esterase protein which possessed a 16 residue insertion relative to the other sequences much like the one present in ORF1.

\section{Key to abbreviations}

Gliotactin Drosophila gliotactin

Lipase Geotrichum lipase 1

Neuroligin1 Drosophila neuroligin1

Neuroligin2 Drosophila neuroligin2

Neuroligin3 Drosophila neuroligin3

Neurotactin Drosophila neurotactin

Glutactin Drosophila glutactin

JHE insect juvenile hormone esterase

DroEst6 Drosophila esterase 6

DroAChE

TorpAChE

HumBChE

Thyroglob

Carbox Est

MsomEst1
Drosophila AChE

Torpedo fish AChE

Human Butyrlcholine esterase

Bovine thyroglobulin

rat carboxylesterase 3

rabbit microsomal esterase 2
Auld et al, 1995

sw:P17573

Ichtchenko et al, 1995

Ichtchenko et al, 1996

Ichtchenko et al, 1996

Escalera et al, 1990

Olson et al, 1990

sw:P12992

sw:P08171

sw:P07140

sw:P04058

sw:P06276

sw:P01267

sw:Q63108

pir1:A34329 


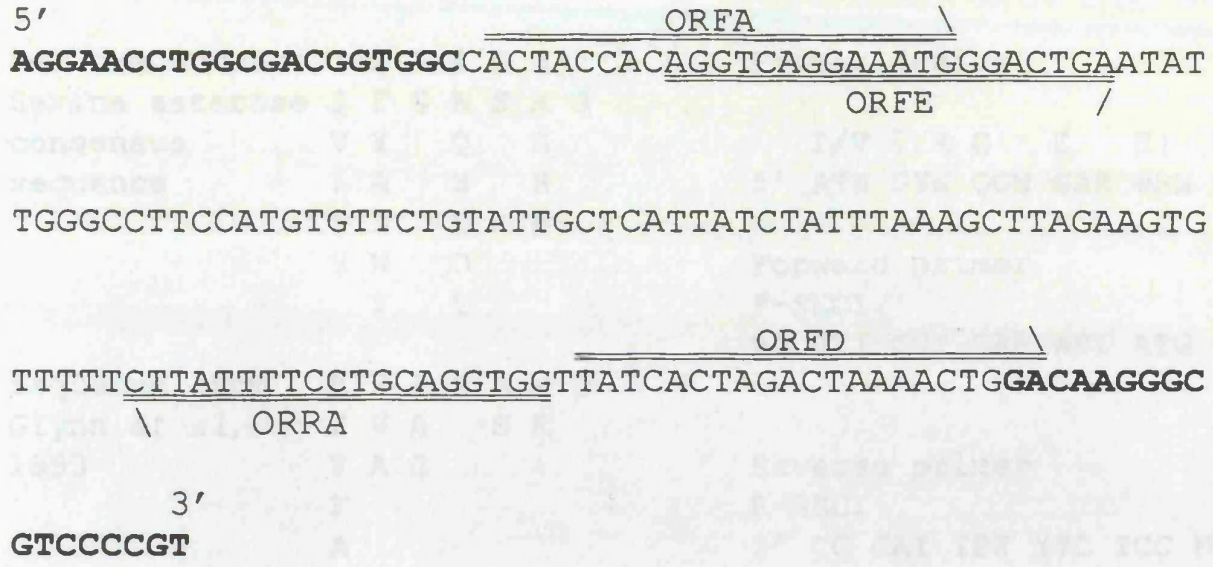

Figure 5.6 ORFA, ORFD, ORFE and ORRA primer locations on ORF1 sequence.

The DNA sequence derived from the degenerate primers is shown in bold. ORFA and ORFE are two of the primers used in the unsuccessful $3^{\prime}$ RACE attempts at cloning more of the CDNA template which gave rise to ORF1.

The ORFD and ORRA were used in the successful inverse PCR of the ORF 1 template. 
Residue number $\begin{array}{lllllll}1 & 2 & 3 & 4 & 5 & 6 & 7\end{array}$ Serine esterase I F G E S A G consensus $\quad V Y$ Q $S$

sequence $\quad \mathrm{I} A \mathrm{H} \quad \mathrm{H}$

$\begin{array}{llll}T & S & S & M\end{array}$

$S W \quad D$

V Y

Sequence from $K E K E A M G$

Glynn et al, I V A S K

1993

$\mathrm{V} A \mathrm{G}$

F

A

Common

Residues

\section{$\underline{\text { Primer design }}$}

$I / V \quad V / A \quad G \quad E \quad S \quad M \quad G$

5' RTN GYN GGN GAR WSN ATG GGN 3'

Forward primer

F-SECI

5' GYI GGI GAR WSI ATG GG 3'

Reverse primer

R-SECI

5' CC CAT ISW YTC ICC NRC 3'

Figure 5.7 Inferred consensus sequence flanking the catalytic serine in NTE. 

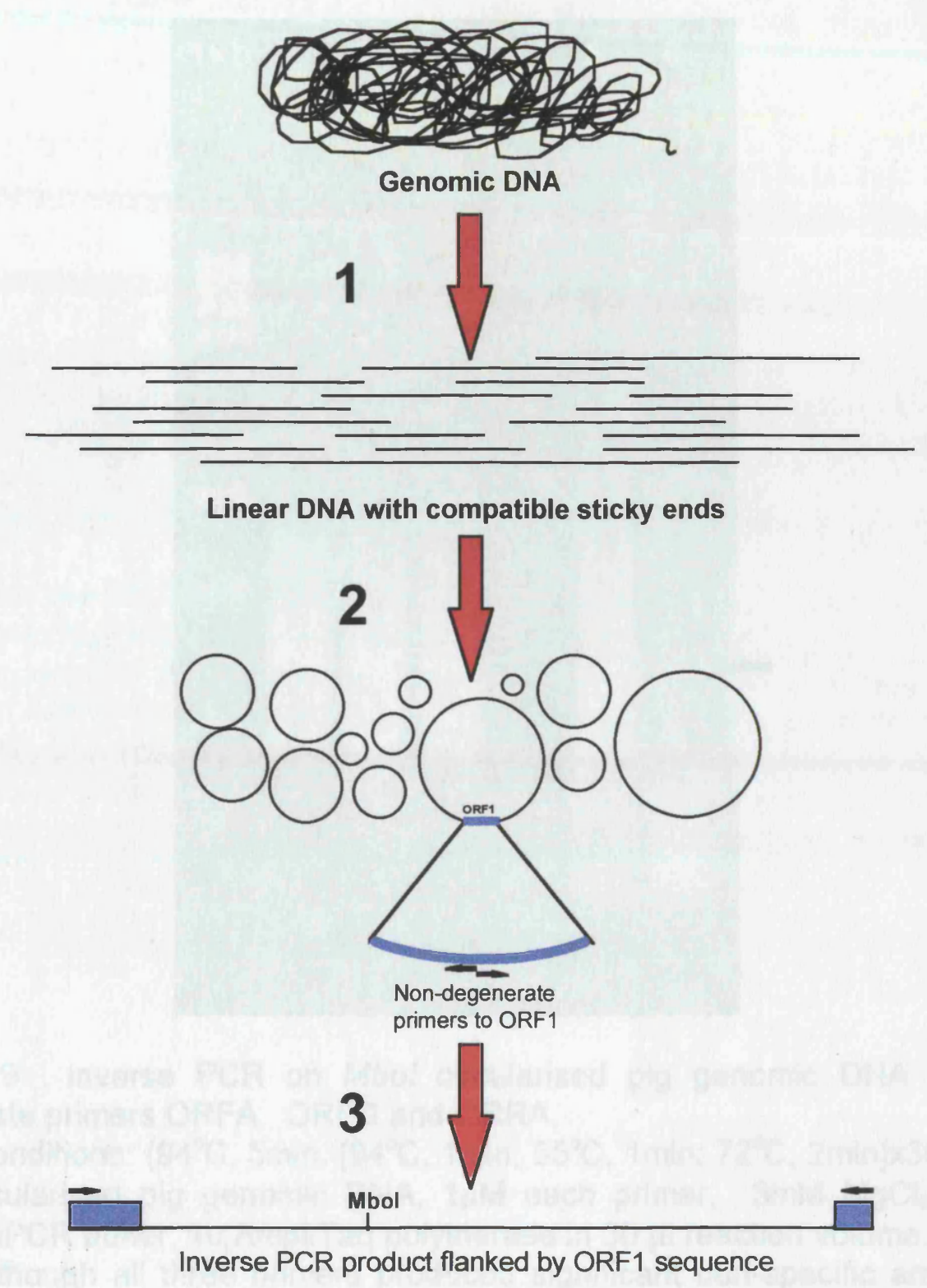

Figure 5.8 Inverse PCR allows amplification of DNA sequences flanking a small section of known sequence.

1) Genomic DNA is digested with a restriction enzyme (in this case Mbol) to produce a population of DNA fragments with identical sticky ends.

2) The digested DNA is religated at high dilution to ensure the fragments self ligate rather than randomly reassociate with other fragments.

3) PCR with primers pointing outwards towards the unknown (flanking) sequence amplifies the bulk of the circle resulting in a novel sequence flanked by the known (ORF1) sequence. 


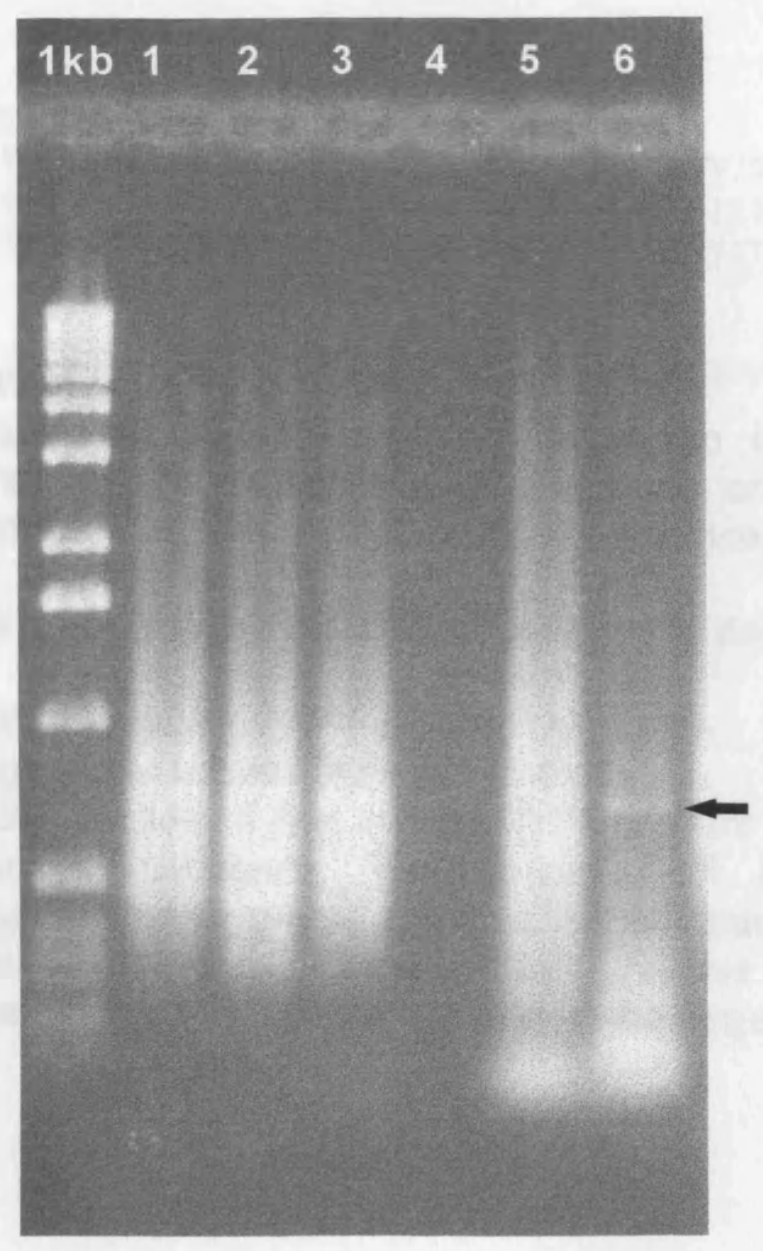

Figure 5.9 Inverse PCR on Mbol circularised pig genomic DNA with nondegenerate primers ORFA, ORFD and ORRA.

Conditions: $\left(94^{\circ} \mathrm{C}, 5 \mathrm{~min},\left[94^{\circ} \mathrm{C}, 1 \mathrm{~min} ; 55^{\circ} \mathrm{C}, 1 \mathrm{~min} ; 72^{\circ} \mathrm{C}, 2 \mathrm{~min}\right] \times 35\right), 300 \mathrm{ng}$ Mbol-circularised pig genomic DNA, $1 \mu \mathrm{M}$ each primer, $3 \mathrm{mM} \mathrm{MgCl}, 0.2 \mathrm{mM}$ dNTP, 1xPCR buffer, 1u AmpliTaq polymerase in $30 \mu$ reaction volume.

Although all three primers produced significant non-specific amplification when alone (lanes 1, 2 and 3), and the ORFA vs ORRA positive control pair also produced a large smear, the ORFD and ORRA pair produce a discrete band of $800 \mathrm{bp}$ in lane 6 (arrowed).

\begin{tabular}{|c|c|c|c|}
\hline Lane & Primers & Lane & Primers \\
\hline 1 & ORFA alone & 4 & No primer blank \\
\hline 2 & ORRA alone & 5 & ORFA vs ORRA \\
\hline 3 & ORFD alone & 6 & ORFD vs ORRA \\
\hline $1 \mathrm{~kb}$ & Gibco $1 \mathrm{~kb}$ ladder & & \\
\hline
\end{tabular}




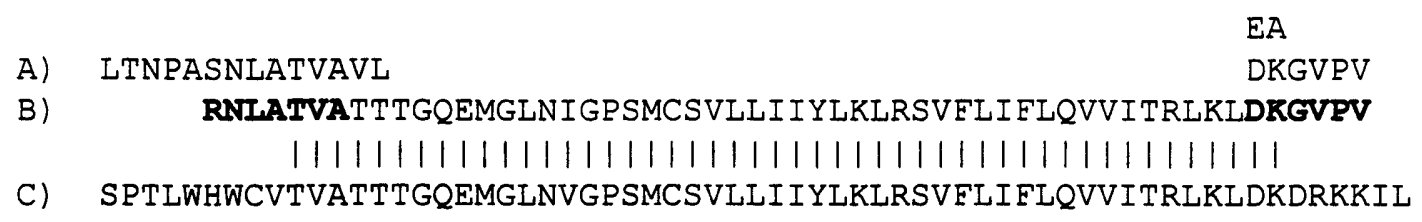

Figure 5.10 Alignment of ORF1 templates.

Only the translated peptide sequence is shown in this figure as it conveniently summarises the relationship between the original NTE peptide sequences LTNP and XGVP, the translated ORF1 sequence, and the translated genomic clone.
A) Represents the original peptide sequences used to design the degenerate primers.
B) Represents the deduced ORF1 peptide sequence. Peptide sequenced derived from the degenerate primers is shown in bold.
C) Represents the section of the inverse PCR product homologous to the deduced ORF1 protein sequence. The inverse PCR product has been reassembled into the original templates configuration and translated to show that the homology between ORF1 and the template that gave rise to it only extends to the first two or three residues ( 6 to 9 base pairs) of the degenerate primers that produced it. 


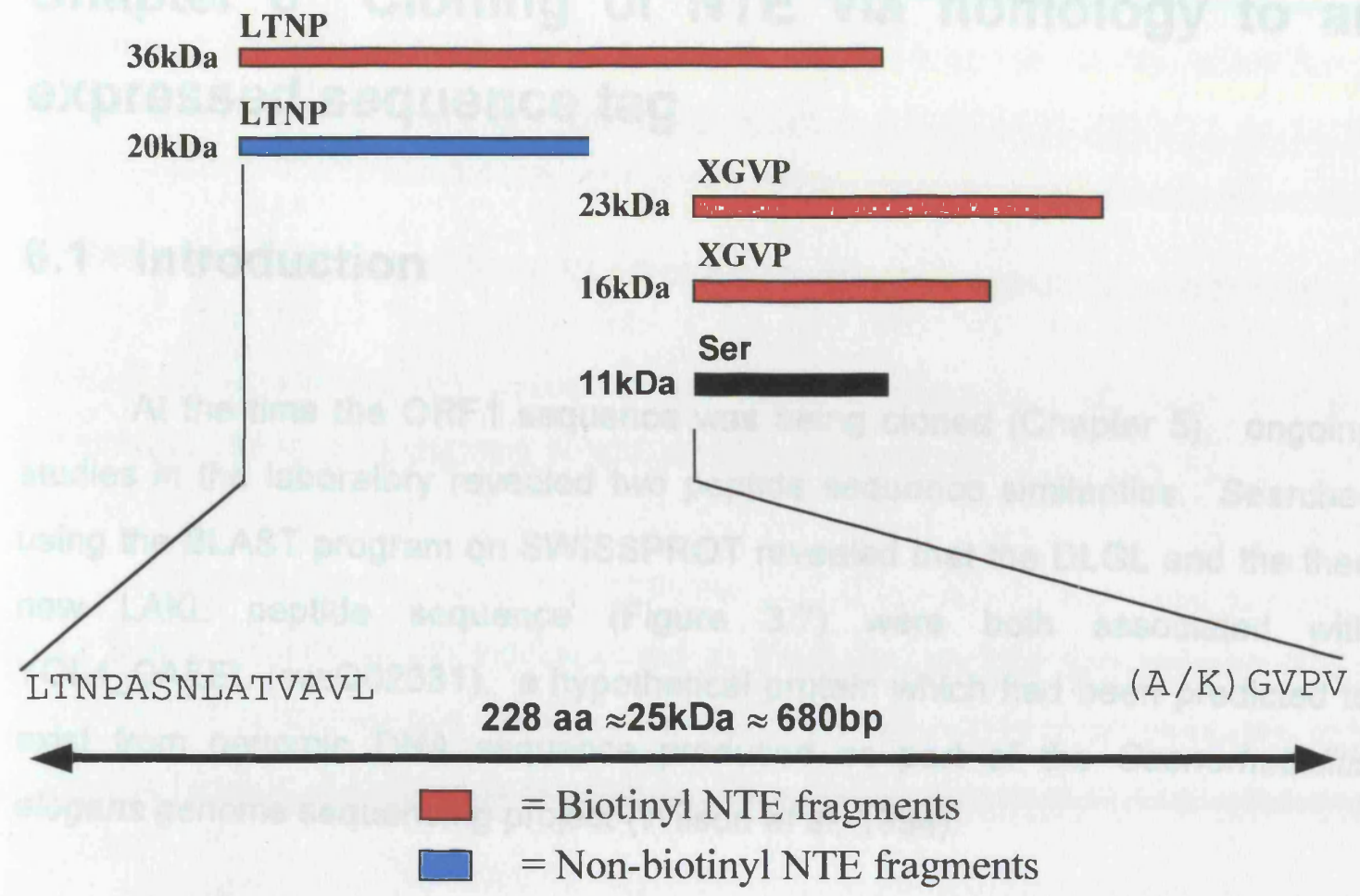

Figure 5.11 Peptide map around the S9B-labelled serine of NTE, using sequence data from Figure 7.1.

The S9B-labelled serine must lie in an $11 \mathrm{kDa}(100 \mathrm{aa})$ interval (shown in black) upstream of the C-terminal end of the $36 \mathrm{kDa}$ fragments and downstream of the XGVP sequence. 


\section{Chapter 6 Cloning of NTE via homology to an expressed sequence tag}

\subsection{Introduction}

At the time the ORF1 sequence was being cloned (Chapter 5), ongoing studies in the laboratory revealed two peptide sequence similarities. Searches using the BLAST program on SWISSPROT revealed that the DLGL and the then new LAKL peptide sequence (Figure 3.7) were both associated with YOL4_CAEEL (sw:Q02331), a hypothetical protein which had been predicted to exist from genomic DNA sequence produced as part of the Caenorhabiditis elegans genome sequencing project (Wilson et al, 1994).

The LAKL peptide sequence had a weak similarity with part of the $\mathrm{N}$ terminal region of YOL4. It also had similarities with Ribulose Bisphosphate Carboxylase (RUBISCO) Large Chain, a 485 amino acid non-biotinylated plant carboxylase which showed that the LAKL/YOL4 match was not of unique quality (Figure 6.1). The DLGL sequence had shown similarity with a group of five overlapping human Expressed Sequence Tag (EST) sequences (R17727, H10469, T10299, H58564 and R51195). Translations of these ESTs were in turn similar to the C-terminal region of YOL4_CAEEL. However DLGL also had a similarity with Escherichia coli acetyl-coenzyme A carboxylase (sw:P08193), a 345 residue biotinylated protein. This match suggested DLGL may not have derived from NTE but a contaminant sequence in the NTE protein preparation (see Section 3.3 and Figure 6.1).

YOL4 is a $152 \mathrm{kDa}$ (1351 residue) hypothetical protein from C. elegans chromosome III (Wilson et al, 1994). At the time there were only two similar database records: YCHK_ECOLI, an Escherichia coli 314 amino acid (34 kDa) hypothetical protein (sw:P37053) and a near identical 178 amino acid fragment from a partial hypothetical protein sequence from Shigella flexneri YCHK_SHIFL 
(sw:P37054; Figure 6.2). On examination, neither the YOL4 nor the YCHK sequences showed any significant similarity with any member of the AChE family of serine-esterases, or any other protein sequence in SWISSPROT or EMBL databases.

One of the ESTs that matched DLGL (em:H58564) was ordered from the IMAGE consortium cDNA collection at Research Genetics Inc, USA and the match was further investigated by the group in three ways:-

1) Deduced peptide sequence from the EST (Figure 6.1) was used to design a synthetic peptide which was coupled to Keyhole Limpet Haemocyanin (KLH) and used to immunise two rabbits. The antibody did not become available until after the peptide sequence produced by Tony Willis (Figure 3.9) had refocused interest in YOL4.

2) The EST was used to screen a $\lambda D R 2$ adult human brain $C D N A$ library and a single $2.2 \mathrm{kbp}$ cDNA insert was isolated. This sequence was later named HOL4.2.

3) The EST was used to probe a human brain mRNA Northern blot which produced a very faint band at about $2 \mathrm{~kb}$.

Combining the data from the similarity searches which indicated DLGL was similar to a known biotinylated protein (Figure 6.1) and the $2 \mathrm{~kb}$ size of the mRNA measured on the Northern blot (much too small to encode the full NTE gene), it was concluded that the DLGL sequence was probably from a contaminating carboxylase protein and the similarity between DLGL, LAKL and YOL4 was just a coincidence and so work on YOL4-connection was suspended.

Interest in YOL4 was revived by the arrival of five new protein sequences (Section 3.3.2. and Figure 3.9). Similarity searches with the new peptides revealed striking links with the YOL4 hypothetical protein. DLGL was found to 
be on the same EST (em:R51195) as the new DGHL sequence and the WKS sequence was located right next to FDQI on another EST (em:R54769, Figure 6.3). In addition to the sequence similarities, the peptide antibody made using the EST based sequence (Figure 6.1) was found to be specific to partially-purified S9B-labelled NTE on a Western blot. These new data indicated that the human ESTs could code for part of the NTE gene.

The homologies between NTE, YOL4 and YCHK and their similarity with several other hypothetical proteins will be discussed in Chapter 7).

\subsection{Results and discussion}

\subsubsection{Sequencing the 3' end of NTE CDNA by alignment of ESTs}

As mentioned in Chapter 1 human ESTs normally come in pairs (one from the $5^{\prime}$ and one from the $3^{\prime}$ ends of the insert; see Figure 1.10 (and Section 1.2.5), the 3 ' ends of a particular transcript are normally the same because the CDNA was primed off the poly-A tail, thus each priming starts from more or less the same place in the transcript. This is useful as a single 3' EST sequence can be used to search the database and identify other clones from the same transcript, then the corresponding 5' sequence which contains DNA sequence from the coding region of the gene can be retrieved from the database and aligned to determine a preliminary consensus sequence for the gene without recourse to further DNA sequencing.

A total of $26 \mathrm{EST}$ clones were recovered from the EST division of EMBL, in addition to this 3 ' and 5 ' sequence from the HOL4.2 (isolated from the $\lambda D R 2$ human brain cDNA library) insert was also generated from cycle sequencing from vector primers. The 5'EST sequences were aligned by looking for sequence overlaps between sequences and also by reference similarities between YOL4 protein sequence and translations of the EST sequences. The completed 'virtual' 
sequence covered a region of $2.2 \mathrm{kbp}$ broken by a single gap of approximately 50bp for which no EST sequence was available. This preliminary DNA consensus sequence contained unavoidable insertions and deletions (despite careful alignment), as the sequences it was based on were derived from automatically generated single pass sequencing runs. However, it was possible to patch together parts of the reading frame by searching for parts of the translated sequence that matched the known NTE peptide sequences (see Figure 7.1) and/or YOL4 sequence (the full EST contig is listed in Appendix 1). This putative protein sequence allowed analysis of the C-terminus well before cycle sequencing of the clone was completed, and was instrumental in showing that the HOL4.2 clone was genuinely from NTE. Aside from allowing a preview of the protein sequence, the EST alignment allowed a series of dye terminator sequencing primers to be designed spaced throughout the HOL4.2 insert and directly confirm the EST based sequence. HOL4.2 is clearly a truncated NTE cDNA, 2200bp of DNA sequence is only enough to encode $730 \mathrm{aa}(\sim 80 \mathrm{kDa})$ of protein sequence as the polyadenylation signal and poly-A tail is present at the $3^{\prime}$ end of HOL4.2, at least another $2 \mathrm{kbp}(\sim 680 \mathrm{aa}, \sim 75 \mathrm{kDa})$ of CDNA sequence would be required to complete the gene (cloning of the 5'end of NTE is descried in Section 6.2.3).

\subsubsection{Subcloning of the $2 \mathrm{kbp}$ insert from pDR2 into pBluescript SK+}

Due to its large size (9.1kbp) the pDR2 vector proved to be a poor subject for purification and sequencing. Thus the first stage in sequencing the gene was to subclone the HOL4.2 insert into a more tractable vector. The HOL4.2 insert was excised from pDR2 with a BamHIX Xbal double digest and agarose gel purified, then subcloned into BamHIXXbal digested pBluescript SK+ (Section 2.2.7). One clone was selected and sequenced using both dye primer and dye terminator sequencing reactions using the primers based on the EST sequence; the final HOL4.2 sequence is shown in Appendix 4. 


\subsubsection{Cloning of the 5' end of NTE by 5' RACE}

As mentioned in Section 6.2.1 the HOL4.2 insert was $2.2 \mathrm{kbp}$ long, enough to encode 730aa of proteins sequence To clone the remaining sequence the initial cloning effort concentrated on demonstrating that the $\lambda D R 2$ human brain CDNA library contained no larger NTE clones. Three antisense primers (NDR2, NDR3 and NDR4) were designed using version 5.0 OLIGO for Windows separated by approximately $100 \mathrm{bp}$ and selected for compatibility with a novel pair of forward primers designed to the pDR2 vector sequence, DRA and DRB (see Figure 6.4). The 100bp separation between the NDR primers was designed to help in identifying the correct products as genuine bands produced from NTE cDNA would differ in size depending on which primer was used. Most nonspecific products would not display this feature and thus could be ignored.

The $\lambda D R 2$ cDNA library was amplified (see Section 2.3.6) to produce sufficient amounts of library phage for repeated PCR experiments and approximately $10^{7}$ plaque forming units (pfu) of phage was used in each reaction (boiled prior to the PCR to destroy DNase activity). Subsequently all permutations of gene specific (NDR2/3/4) and vector specific (DRA/B) primers were tested searching for PCR products larger than those produced by HOL4.2 insert in PDR2 vector (Figure 6.5).

Once it became clear that the $\lambda D R 2$ cDNA library contained no full length inserts which could be amplified by PCR (a result later confirmed by conventional hybridisation screening performed by other members of the research group), 15 ng of Marathon human brain RACE ready CDNA was procured from Clontech and utilised in a series of 5 ' RACE reactions.

Figure 6.6 summarises the principal features of the Marathon cDNA system. Of particular note is the use of the $T 7$ promoter sequence as the anchor primer (AP1). The pBluescript SK+ vector contains the T7 promoter and fortuitously it adjoined the 5 ' end of the HOL4.2 insert in the subcloned construct 
described previously. Thus the NDR2 and 4 primers could be tested on a known template, and it was demonstrated that the NDR2/4 primers were compatible with the Marathon AP1 primer (data not shown).

Initial experiments used Pfu DNA polymerase because of its proof-reading activity and its ability to amplify large templates (Lundberg et al, 1991). Using the pBluescript/HOL4.2 construct as a primer positive control and a glyceraldehyde-3-phosphate dehydrogenase (GAP-DH) specific primer as a CDNA template control, a set of PCR conditions were determined which would efficiently amplify both the control DNAs. However when these conditions were used on the Marathon CDNA only a smear was produced, even when nested PCR was attempted. The smearing of the product was finally overcome using by changing the polymerase to Clontech Advantage KlenTaq. This produced a discrete band of about $2.9 \mathrm{kbp}$ from the NDR4 primer and a slightly smaller band from the NDR2 primer (Figure 6.7).

Previously the Stratagene pCRscript cloning system was the method of choice for blunt end DNA cloning. However the PCRscript system was unsuitable for cloning the 5' RACE product since the Clontech linker adapter contained a Srfl restriction site. The pCRscript kit selects for recombinant plasmids by including Srfl (a blunt ending restriction enzyme with an $8 \mathrm{bp}$ recognition sequence 5' GCCC'GGGC 3') into the ligation mixture. The pCRscript plasmid contains a Srfl restriction site which is also the insertion site for the PCR product, thus non-recombinant $p C R s c r i p t$ is maintained in the linear state. When an insert ligates into the vector, the Srfl site is disrupted so the vector remains in a circular form which can transform the competent cells (Stratagene, 1996).

A certain amount of difficulty was encountered in cloning the $2.9 \mathrm{kbp}$ insert since limited amounts of the PCR product were available. The cloning problem was finally resolved by resynthesising the NDR2 and NDR4 primers and adding a $X$ hol site to the 5 ' end to make the primers XNDR2 and XNDR4 (Figure 6.4). The products from the 5' RACE reaction using these new primers were digested with 
Not/ $X \mathrm{Xhol}$ and ligated into Not//Sall digested pBluescript SK+ vector, which had been dephosphorylated with Calf Intestinal Alkaline Phosphatase (CIAP). Subsequently the ligation mix was transformed into Stratagene supercompetent XL1 Blue MRF' cells (see Sections 2.2.7 and 2.2.10).

Colony lifts were taken from recombinants and screened using the first $200 \mathrm{bp}$ of the HOL4.2 insert (from the $5^{\prime}$ end to NDR4 primer) labelled with ${ }^{32} \mathrm{P}$. One hundred and twenty two positive clones were selected for further investigation by PCR amplification between the vector primer RSP and NDR4 (Figure 6.8). The largest 14 clones were selected and dye primer sequenced (Section 2.3.2) from the vector sequences.

\subsubsection{Sequencing of the 5' RACE products}

Examination of the $145^{\prime}$-sequences showed that each was derived from independent CDNA transcripts as the initial DNA sequence was different in each clone (see Figure 6.9). However, when aligned they formed two groups of sequences which were named NTE1 and NTE2. Representatives from both these groups have been partially or fully sequenced using primers designed to the D16 NTE clone which had been produced other work by the research group (see Section 6.3).

Only one of the NTE1 clones has so far been sequenced in full (a28); it consists of a 2607bp sequence (Appendix 4) which has a 266bp overlap with the HOL4.2 DNA sequence (Figure 6.10), A contig of a28 and HOL4.2 (a28HOL4) contains one open reading frame (ORF) of 1342aa. The likely start codon is located 16aa into the ORF (Figure 6.11) to give a 1327aa protein (Appendix 4) with a predicted molecular weight of $146 \mathrm{kDa}$, close to the $155 \mathrm{kDa}$ measured for NTE. Partial sequence data from the other NTE1 clone (a12) has been compared to the a28 sequence and found to be almost identical. 
The NTE2 sequence group, contains three clones which have been partially sequenced and show evidence of alternate splicing at the 5' end (Figure 6.11). The remaining cDNAs are truncated and start more than 500bp from the beginning of the a28 clone and thus match both NTE1 and NTE2.

\subsection{Review of gene cloning}

Once it became clear that HOL4.2 insert encoded a portion of NTE CDNA sequence (see Section 6.1 and Figure 7.1) two approaches were taken to obtaining the 5' end of the gene: 5' RACE (described above) and Hybridisation screening of human brain CDNA libraries (undertaken by other members of the research group). The hybridisation screening started by rescreening the $\lambda D R 2$ human brain which had originally yielded the HOL4.2 clone. No larger CDNA clones were detected (confirming the 5' RACE screening of the library; Section 6.2.3). Subsequent screening of a foetal brain CDNA library (kindly provided by W. Schwaeble, University of Leicester), produced four CDNA clones three of which have been partially sequenced and are not full length clones. The translation product of the remaining clone, D16, is identical to that of the contig a28HOL4. This sequence is analysed fully in Chapter7. 


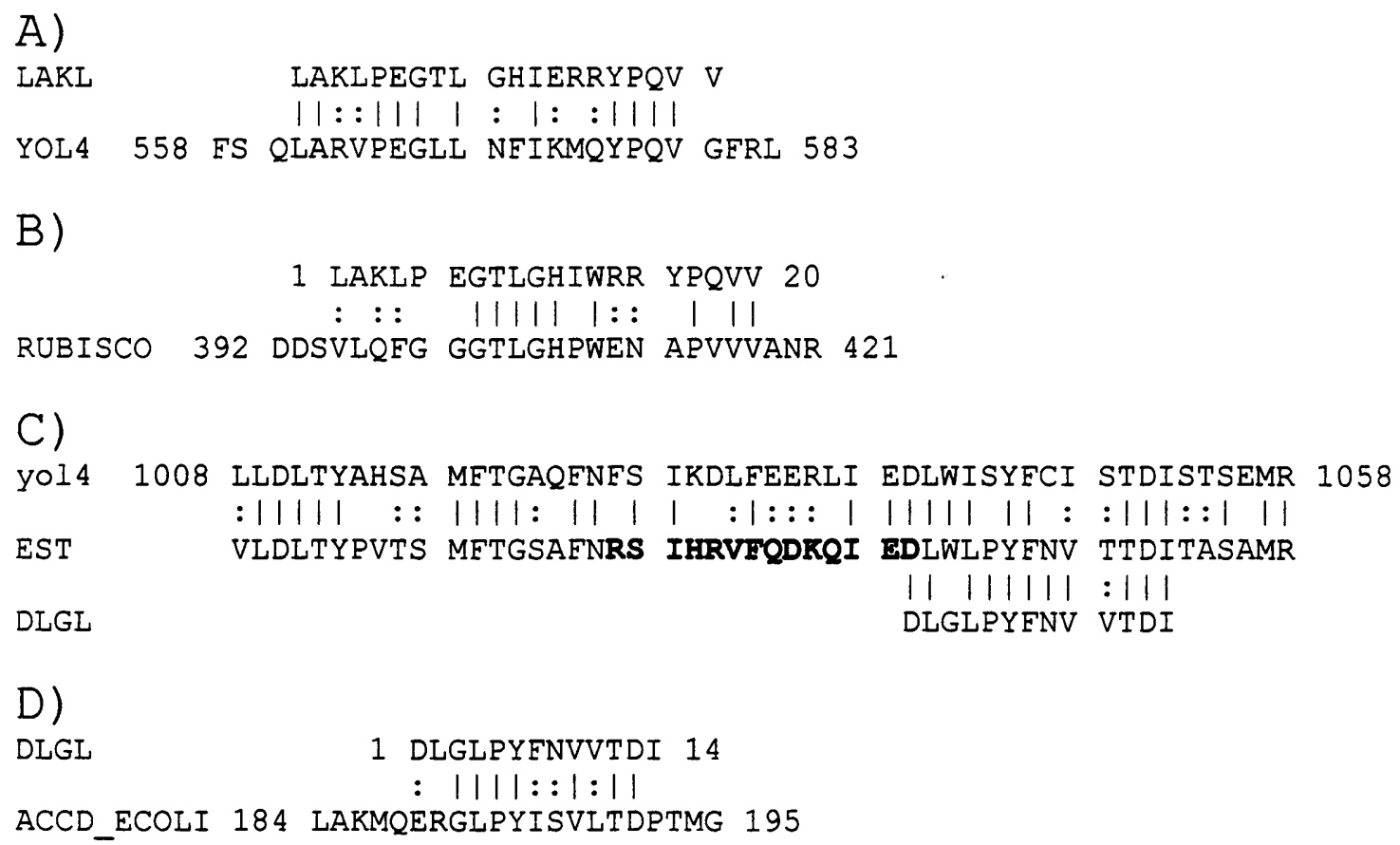

D)

DLGL

Figure 6.1 Similarity between the pig NTE peptide sequence LAKL and YOL4_CAEEL, LAKL and RUBISCO, DLGL and YOL4_CAEEL, DLGL and Acetyl-CoA carboxylase.

A) The LAKL sequence was moderately similar to the hypothetical C. elegans protein YOL4. No human EST sequences were available for this region of the protein.

B) LAKL was also similar to RBL_HELAN (the large chain of Sunflower Ribulose bisphosphate carboxylase (RUBISCO, sw:P45738).

C) The DLGL sequence was similar to the translated peptide sequence of EST H58564 and YOL4 hypothetical peptide sequence. Sequence shown in bold was used to make a peptide antibody (Section 6.1).

D) The DLGL sequence also has a similarity with $E$. coli acetyl-coenzyme $A$ carboxylase carboxyl transferase subunit beta (sw:P08193). This cast some doubt on the authenticity of the sequence.

$1=$ identity, : = similarity (amino acid pair has a score of 0 or greater on the BLOSUM62 matrix. Henikoff and Henikoff, 1992). 
1

yol 4 caeel ychk_ecoli ychk_shifl

yol4_caeel ychk ecoli ychk_shifl

yol 4 caeel ychk_ecoli ychk_shifl

yol 4 caeel ychk_ecoli ychk_shifl

yol4_caeel ychk ecoli ychk_shifl

yol4_caeel ychk_ecoli

yol4 caeel ychk_ecoli
FWTPDRRSDF SRLARILTGN AIGLVLGGGG ARGAAHVGVL RALREEGIPV MATIAEQGNL AGIMRKI... KIGLALGSGA ARGWSHIGVI NALKKVGIEI

$51 \quad 100$

DIVGGTSIGS LIGGLYAETP DDVVVETRAA SWFNGM.SSL WRKLLDLTYA DIVAGCSIGS LVGAAY..... . ACDRLSALE DWVTSFSYWD VLRLMDLSWQ

101

HSAMFTGAQF NFSIKDLFEE RLIEDLWISY FCISTDISTS EMRVHRSGPL RGGLLRGERV FNQYREIMPE TEIENCSRRF AAVATNLSTG RELWETEGDL

15 200

WAYCRASMSL AGYLPPLCDP QDGHLLLDGG YVNNVPADVM RNLGARCVIA HLAIRASCSI PGLMAPV.. A HNGYWLVDGA VVNPIPISLT RALGADIVIA

$201 \quad 250$

CDVG.SIEET NLYDYG...........DSL SGMWVLLKRL NPFGTPPRIL VDLQHDAHLM QQDLLSENVS EENSENGDSL PWHARLKERL GSITTRRAVT $--$

251 300

NMEEIQSRLA YVSCVRQLEV VKKASYCRYL RPPIEPEKTL DFPKFQEIME APTATEIMTT SIQVLENR.. LKRNRMAGDP PDILIQPVCP QISTLDFHRA

301 350

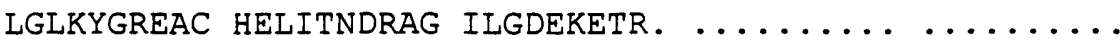

HAAIAAGQLA VERKMDELLP IVRTNI ... ............

Figure 6.2 Alignment between YCHK_ECOLI, YCHK_SHFTL, and the Cterminal domain of YOL4_CAEEL.

The fragmentary YCHK_SHFTL sequence was identical to YCHK_ECOLI (shown as - in the SHFTL sequence) except in 4 positions (shown in bold). 
EST R51195 Reading frame +1

$\begin{array}{lr}\text { DLGLPYFNVVTDI } & \text { DGHLLTDGGYINNLP } \\ 11111111: 111 & 11111111111111 \\ \text { IEDLWLPYENVTTDITASAMRVHKDGSLWRYVRAXMDAVGLPAPAVXPDGHLLMDGGYINNLPADI } & 67 \\ 1 & \end{array}$

\section{EST R54769 Reading frame +3}

VVKSSS?DG?LR??IDYFK FDQIYDVGYQYGK

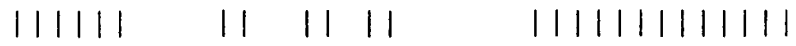

QLEVVKSSSYCEYLRPPIDCFKTMDFGKFDQIYDVGYQYGKAVFG

$\begin{array}{ll}1 & 45\end{array}$

Figure 6.3 Similarity between translated EST sequences and the DLGL, DGHL, WKS and FDQI peptide sequences. 


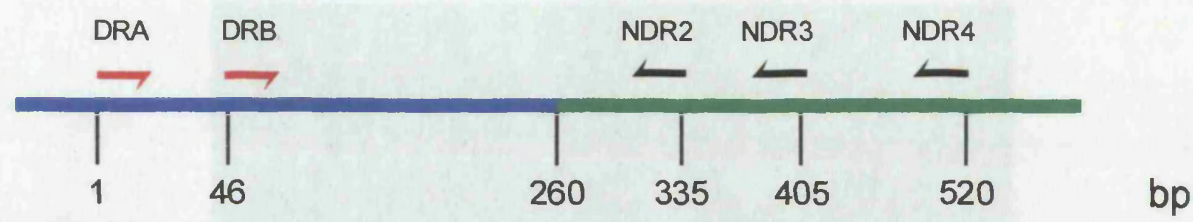

5 ' end of HOL4.2 (NTE) insert pDR2 vector sequence

Figure 6.4 Relative positions of primers in insert and pDR2 vector sequence at the 5 ' end of the HOL4.2 insert.

Primer DRA is located 493bp from the start of the published pDR sequence (em:U02428).

Primers XNDR2 and XNDR4 were used later in the project and include a $X$ hol site at the $5^{\prime}$ end (shown in bold) to aid cloning.
Primer sequence
$5^{\prime}----->3$ '
DRA CGTGCATGCCGATTGGTG
DRB ATACAATAAACGCCATTTGA
NDR2 TTGGTGAGTTCCGAGTGTGG
NDR3 GCGTGAAGGCCACCA
NDR4 ACAGCCGGAACTCTTGGATG
XNDR2 CCGCTCGAGTTGGTGAGTTCCGAGTGTGG
XNDR4 CCGCTCGAGACAGCCGGAACTCTTGGATG 


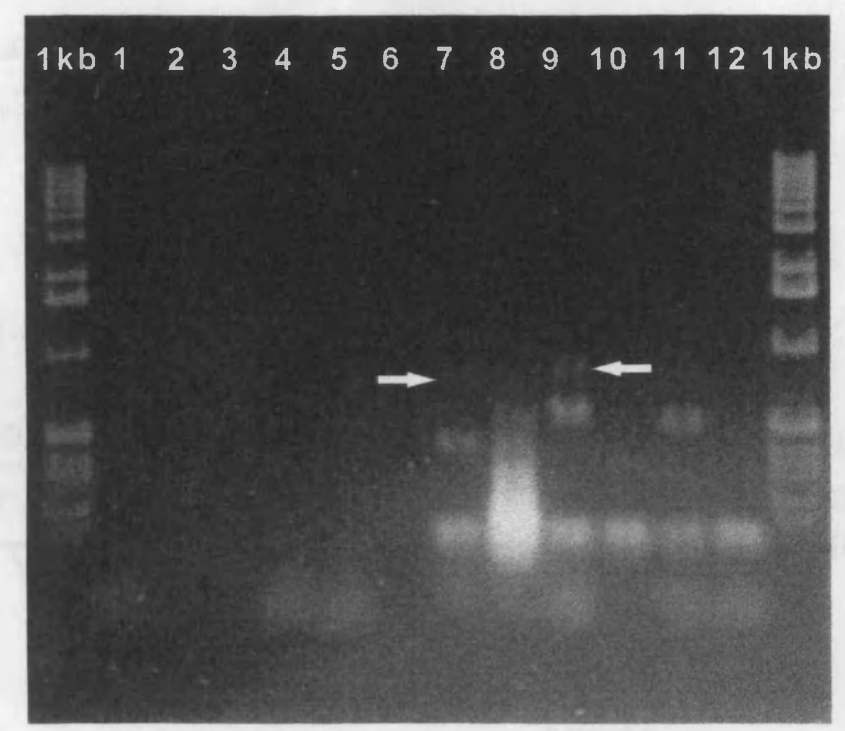

Figure 6.5 PCR of DRAVB primers vs NDR2/3/4 on $\lambda D R 2$ adult human brain cDNA library.

Conditions $\left(94^{\circ} \mathrm{C}, 5 \mathrm{~min},\left[94^{\circ} \mathrm{C}, 1 \mathrm{~min} ; 68^{\circ} \mathrm{C}, 4 \mathrm{~min}\right] \times 40\right), 10^{7} \mathrm{pfu}$ bacteriophage $2 \mu \mathrm{M}$ each primer, $0.2 \mathrm{mM}$ dNTP, 1xTaq2000 buffer, 1u Taq2000 polymerase in $25 \mu \mathrm{l}$ reaction volume.

Lanes 7 and 9 show bands at about 300 and 500 bp as would be predicted from Figure 6.4. There also appears to be a faint band at about $800 \mathrm{bp}$ in both lanes (arrowed). Although this product could have represented a longer NTE clone, it could not have represented a full length clone (a further 2 to $3 \mathrm{kbp}$ of DNA sequence would be needed to complete NTE cDNA), and so was not pursued.

Lane 8 (DRA vs NDR3) has failed to amplify properly; a similar pattern of products is seen in the negative control (data not shown) so the smear is presumably the result of primer dimer formation.

The DRB primer appears not to be as successful as DRA, only the DRB vs NDR3 primer pair has produced any suitable product and even that indicates the template was no larger than the one already known.

\begin{tabular}{|c|c|c|c|}
\hline Lane & $\underline{\text { Primers }}$ & Lane & Primers \\
\hline 1 & $\overline{\text { DRA alone }}$ & $\overline{7}$ & $\overline{\text { DRA vs NDR2 }}$ \\
\hline 2 & DRB alone & 8 & DRA vs NDR3 \\
\hline 3 & NDR2 alone & 9 & DRA vs NDR4 \\
\hline 4 & NDR3 alone & 10 & DRB vs NDR2 \\
\hline 5 & NDR4 alone & 11 & DRB vs NDR3 \\
\hline 6 & no primer blank & 12 & DRB vs NDR4 \\
\hline $1 \mathrm{~kb}$ & Gibco $1 \mathrm{kbp}$ ladder & & \\
\hline
\end{tabular}




\section{A) Marathon cDNA synthesis primer}

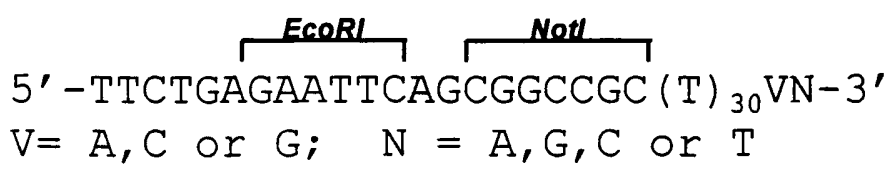

B) Marathon CDNA adaptor

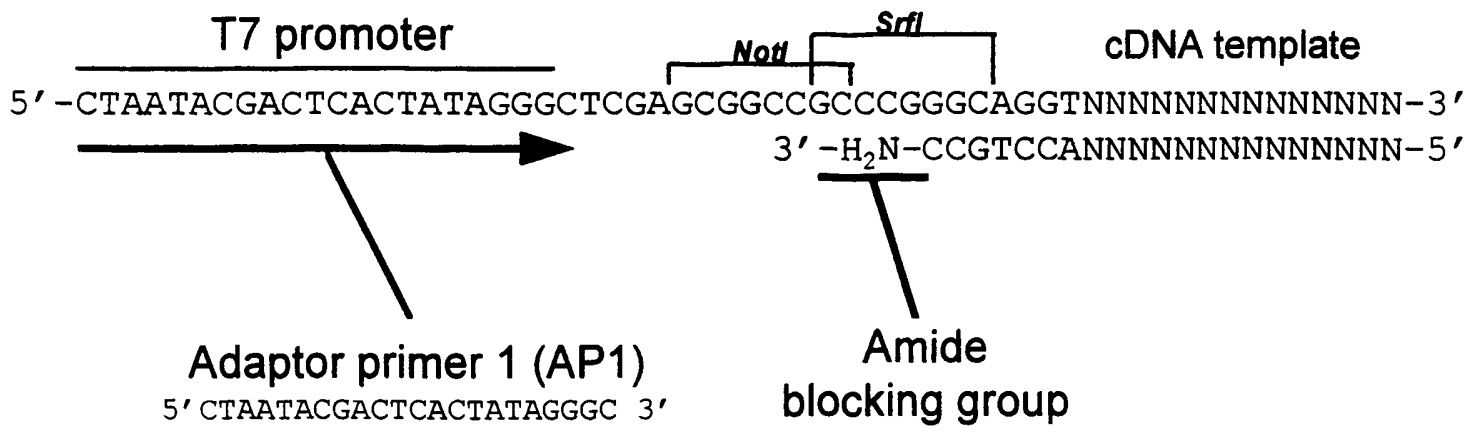

Figure 6.6 Synthesis of Marathon CDNA.

Clontech synthesise the first strand of CDNA using the synthesis primer (A) and Moloney murine leukaemia virus reverse transcriptase. The second strand is made using a cocktail of E. coli DNA polymerase I, RNase $H$ and E. coli DNA ligase. The double stranded cDNA is blunt ended with T4 DNA polymerase and the Marathon CDNA adapter (B) ligated onto both ends of the CDNA template using T4 DNA ligase (Clontech, 1997).

The amide blocking group attached to both $3^{\prime}$ ends ensures that 5 ' overhang is not filled in by the polymerase; thus, when the strands are separated in the first round of PCR, each strand has only one copy of Adapter Primer 1 (AP1) region. Since the CDNA adapter does not have the AP1 complementary sequence, the AP1 primer alone is unable to anneal to the CDNA preventing nonspecific amplification. When a gene specific primer (GSP) anneals to the CDNA and the strand complimentary to the AP1 sequence is produced, the AP1 primer can anneal and PCR amplification can proceed.

Which end of the gene gets amplified is dependent on whether the GSP was designed to anneal to the first (anti-sense) or second strand (sense) CDNA strand; the former would amplify the 3' end of a gene, the latter works on the 5' end strand. 


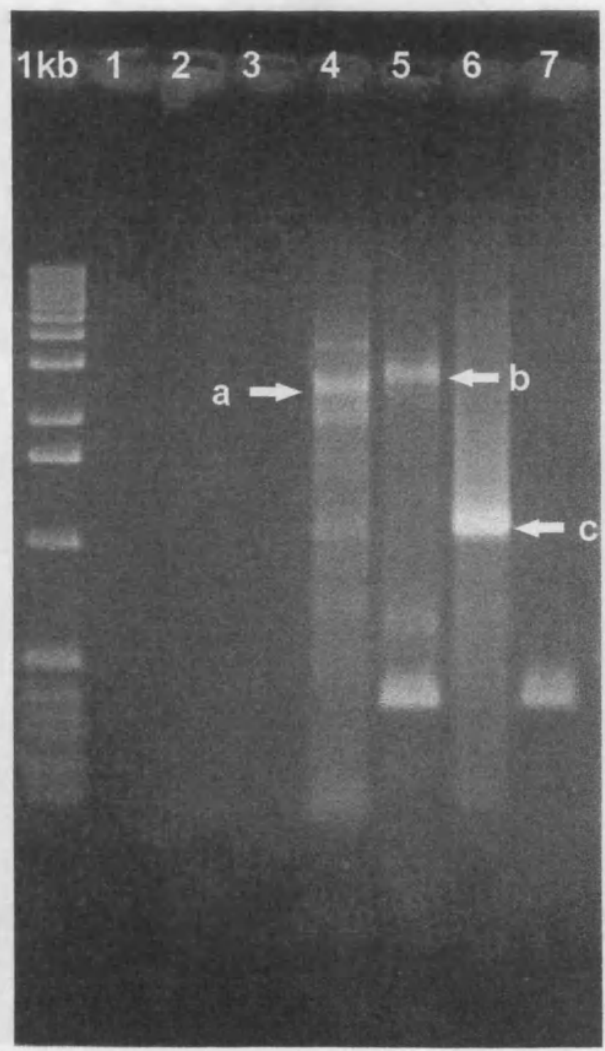

Figure 6.7 PCR amplification using NDR2 and NDR4 vs AP1 primers on Marathon cDNA.

Conditions $\left(94^{\circ} \mathrm{C}, 5 \mathrm{~min},\left[94^{\circ} \mathrm{C}, 1 \mathrm{~min} ; 68^{\circ} \mathrm{C}, 4 \mathrm{~min}\right] \times 30\right), 0.08 \mathrm{ng}$ Marathon cDNA $(2.5 \mu \mathrm{l}), 0.2 \mu \mathrm{M}$ AP1 primer, $0.2 \mu \mathrm{M}$ NDR2/4 primer , $0.2 \mathrm{mM}$ dNTP, 1xKlenTaq PCR buffer, 0.5 units Advantage KlenTaq polymerase in $25 \mu \mathrm{l}$ reaction volume).

The NDR2/AP1 primer pair (lane 4) shows a major band at $\sim 2.7 \mathrm{kbp}$ (a), the NDR4/AP1 primer pair (lane 5) has a major band at $\sim 2.9 \mathrm{kbp}$ (b). These two bands were thought to be significant since they were about the correct size to encode the 5 ' end of the NTE cDNA. Secondly the size difference of the two bands was as predicted from the original design on the NDR primers (Figure 6.4).

Lane 6 shows the $1 \mathrm{kbp}$ band produced by the GAP-DH 5' RACE control primer (5'-TCCACCACCCTGTTGCTGTAG-3') supplied in the Marathon RACE ready cDNA kit.

\begin{tabular}{|c|c|c|c|}
\hline Lane & Primers & Lane & Primers \\
\hline$\overline{1 \mathrm{~kb}}$ & Gibco $1 \mathrm{kbp}$ ladder & $\overline{4}$ & $\overline{\text { AP1 vs NDR2 }}$ \\
\hline 1 & AP1 alone & 5 & AP1 vs NDR4 \\
\hline 2 & NDR2 alone & 6 & AP1 vs GAP-DH \\
\hline 3 & NDR4 alone & 7 & AP1 vs NDR4, no template blank \\
\hline
\end{tabular}




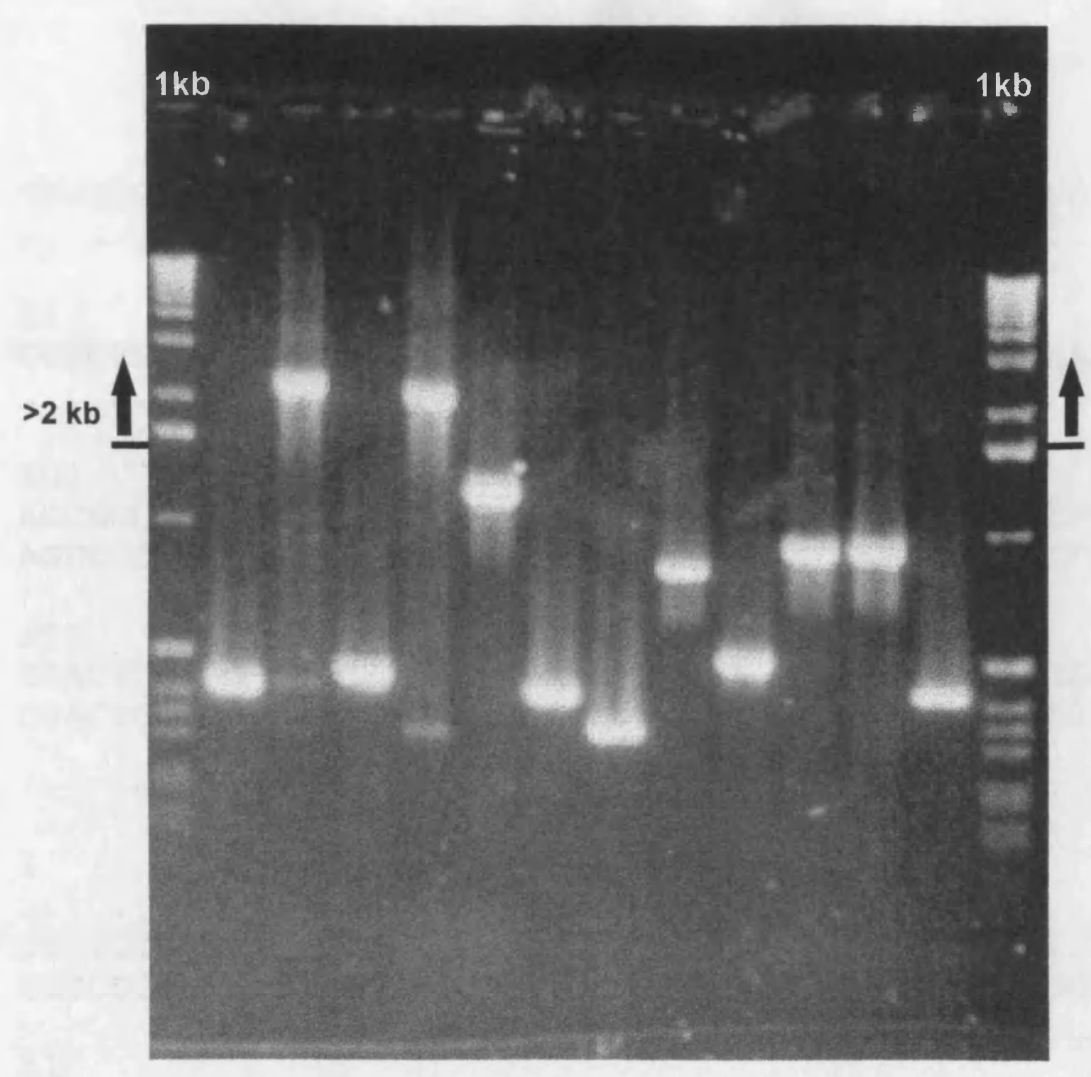

Figure 6.8 PCR with RSP vs NDR4 sizing the clones.

Conditions $\left(94^{\circ} \mathrm{C} 5 \mathrm{~min},\left[94^{\circ} \mathrm{C} 1 \mathrm{~min}, 68^{\circ} \mathrm{C} 4 \mathrm{~min}\right] \times 25\right), 10 \mu$ boiled bacteria, $0.2 \mu \mathrm{M}$ RSP primer, $0.2 \mu \mathrm{M}$ NDR4 primer , $0.2 \mathrm{mM}$ dNTP, 1xKlenTaq PCR buffer, 0.5 units Advantage KlenTaq polymerase in $25 \mu$ l reaction volume).

The RSP primer (5'-GGAAACAGCTATGACCATG-3') is located 85bp from the Notl site used to clone the original PCR product, and pairs with the NDR4 primer to produce a product spanning the full insert length.

Colonies were picked using a $10 \mu$ l pipette tip and the colony placed in 10 $\mu$ of water; about $1 \mu$ l of the water was then spotted out onto LB agar supplemented with $50 \mu \mathrm{g} / \mathrm{ml}$ ampicillin. The bacterial suspension was heated to $100^{\circ} \mathrm{C}$ for 10 minutes then $15 \mu \mathrm{l}$ of PCR reaction mix was added to it.

After amplification the products were resolved on an agarose gel and clones of $2 \mathrm{kbp}$ or larger were identified and the corresponding colony was further cultured to produce sufficient DNA for sequencing. Twelve representative reactions out of 122 are shown in this figure. 


\section{NTE1}

$1 \quad 50$

a28 GTGGCGCCAC CATCGGTCCC GGAGTCCCAG TGATGCTCTG TGCCATAGAG

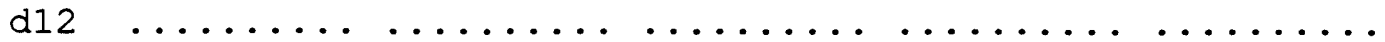

$51 \quad 100$

a28 CCCCCATAAC TTCACTACTA CGTGATAGTA AATCCCCGGC AAAAACCAGC

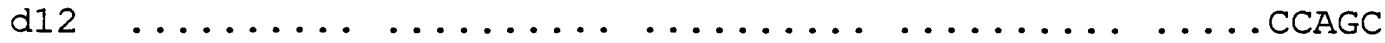

$101 \quad 150$

a28 AGCGCCTTGC AAGCCCACGC CACCCCAAGC ATCCCAGGAC TCTTCTGAAA

d12 AGCGCCTTGC AAGCCCACGC CACCCCAAGC ATCCCAGGAC TCTTCTGAAA

151

200

a28 CGACTCCGGG CTACCAGATC GGCCGTCCAG CTGGAATCAA CCGATGGAGG

d12 CGACTCCGGG CTACCAGATC GGCCGTCCAG CTGGAATCAA CCGATGGAGG

\section{NTE2}

1

d4 1

$\mathrm{d} 43$

GCCCGGGCAG GTCGAGTCAC GGGCTGGCTA CGAACTCCTC GGGGGCGAAG

51

100

$\mathrm{d} 41 \ldots \ldots \ldots \ldots \ldots \ldots$ TT CCAGGACGTC CTGGCGCCCG GGGAAGGCTC

$\mathrm{d} 43 \quad \ldots \ldots \ldots \ldots \ldots \ldots \ldots \ldots \ldots \ldots \ldots \ldots \ldots$. . . . . . . .

a27 GTGGCGGAGA GGGATGGGTT CCAGGACGTC CTGGCGCCCG GGGAAGGCTC

101

150

d41 GGCGGGACGG ATTTGCGGTG CGCAGCCAGT GCCGTTCGTC CCTCAGGTGC

d43 GGCGGGACGG ATTTGCGGTG CGCAGCCAGT GCCGTTCGTC CCTCAGGTGC

a27 GGCGGGACGG ATTTGCGGTG CGCAGCCAGT GCCGTTCGTC CCTCAGGTGC

151

200

d41 TTGGCGTGAT GATCGGGGCC GGAGTGGCGG TGGTGGTCAC GGCCGTGCTC

d43 TTGGCGTGAT GATCGGGGCC GGAGTGGCGG TGGTGGTCAC GGCCGTGCTC

a27 TTGGCGTGAT GATCGGGGCC GGAGTGGCGG TGGTGGTCAC GGCCGTGCTC

Figure 6.95 ' end sequence from the NTE1 and NTE2 product groups.

Two clones match the NTE1 pattern and three clones match the NTE2 pattern; since the reverse transcription reaction has terminated at different points in the sequence, the clones are of different lengths and they must have been amplified from different templates and therefore independent clones. 
NTE1 CCACCTACTG AGCCAGAAAA TTCTAGgGAA TTTGCAGCAG CTGCAAGGAC

HOL $4.2 \ldots \ldots \ldots \ldots \ldots \ldots \ldots \ldots$.

2370

$\downarrow \downarrow \downarrow$

2419

NTE1 CCTTCCCAGC AGGCTCTGGG TTGGGTGTGC CCCCACACTC GGAACTCACC

HOI 4.2 CCTTCCCAG. ..GCTCTGGG TTGGGTGTGC CCCCACACTC GGAACTCACC

$$
2420
$$

NTE1 AACCCAGCCA GCAACCTGGC AACTGTGGCA ATCCTGCCTG TGTGTGCTGA HOL4.2 AACCCAGCCA GCAACCTGGC AACTGTGGCA ATCCTGCCTG TGTgTGCTGA

$$
2470
$$

NTE1 GGTCCCCATG GTGGCCTTCA CGCTGGAGCT GCAGCACGCC CTGCAGGCCA HOL4.2 gGTCCCCATG GTGGCCTTCA CGCTGGAGCT GCAGCACGCC CTGCAGGCCA

$$
2570
$$

NTE1 TCGGTCCGAC GCTACTCCTT AACAGTGACA TCATCCGGGC ACGCCTGGGG HOL4.2 TCGGTCCGAC GCTACTCCTT AACAGTGACA TCATCCGGGC ACGCCTGGGG

\author{
NTE1 GCCTCCGCAC TGGATAGCAT CCAAGAGTTC CGGCTGTC........... \\ HOL4.2 GCCTCCGCAC TGGATAgCAT CCAAGAGTTC CGGCTGTCAG GGTGGCTGGC
}

Figure 6.10 Overlap between the NTE1 clone and HOL4.2 CDNA

The 3bp difference (arrowed) is probably a mutation in HOL4.2 sequence and as the deletion had no effect on the reading frame, the NTE1 sequence was used in the overlap zone. 


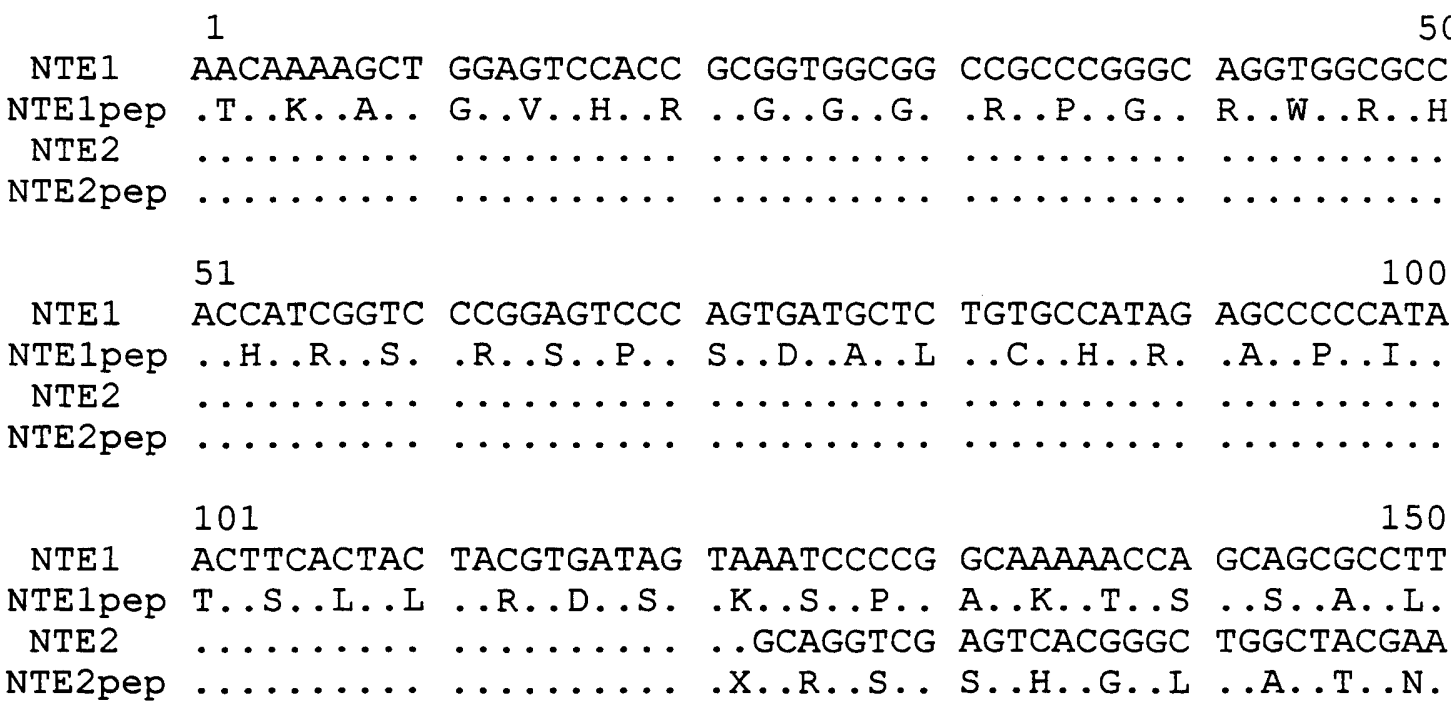

NTE1 AACAAAAGCT GGAGTCCACC GCGGTGGCGG CCGCCCGGGC AGGTGGCGCC NTE1pep .T..K..A.. G..V..H..R ..G..G..G. .R..P..G..R..W..R..H NTE2 51 .A.......

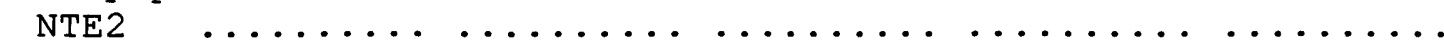

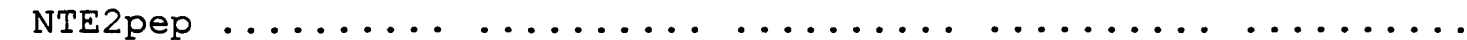
NTElpep T..S..L..L ..R..D..S. .K..S..P.. A..K..T..S ..S..A..L.

$151 \quad 200$

NTE1 GCAAGCCCAC GCCACCCCAA GCATCCCAGG ACTCTTCTGA AACGACTCCG NTElpep .Q..A..H.. A..T..P..S ..I..P..G. .L..F..*.. N..D..S..G NTE2 CTCCTCGGGG GCGAAGGTGG CGGAGAGGGA TGGGTTCCAG GACGTCCTGG NTE2pep .S..S..G.. A..K..V..A ..E..R..D. .G..F..Q.. D..V..I..A

$201 \quad$ ACCATGG 250

NTE1 GGCTACCAGA TCGGCCGTCC AGCTGGAATC AACCGATGGA GGCTCCGCTG NTE1pep ..L..P..D. .R..P..S.. S..W..N..Q ..P..M..E. .A..P..L.. NTE2 CGCCCGGGGA AGGCTCGGCG GGACGGATTT GCGGTGCGCA GCCAGTGCCG NTE2pep ..P..G..E. .G..S..A.. G..R..I..C ..G..A..Q. .P..V..P..

\begin{tabular}{|c|c|c|c|c|c|}
\hline \multirow{4}{*}{$\begin{array}{c}\text { NTE1 } \\
\text { NTE1pep } \\
\text { NTE2 } \\
\text { NTE2pep }\end{array}$} & $\begin{array}{l}251 \ldots \text { ACCA } \\
\text { CAAACTGGAA }\end{array}$ & TGGTGCTTGG & CGTGATGATC & & TGGCGGTGG \\
\hline & Q.T..G..M & .V..I..G. & .V..M..I.. & G.A..G..V & $\ldots A . . V \ldots V$ \\
\hline & TTCGTCCCTC & AGGTGCTTGG & CGTGATGATC & GGGGCCGGAG & TGGCGGTGC \\
\hline & $F \ldots V \ldots P \ldots Q$ & .V. .I..G. & .V..M.... & G.A..G.VV & $\ldots A . . V \ldots V$ \\
\hline & 301. & & & & \\
\hline NTE1 & GGTCACGGCC & GTGCTCATCC & TCCTGGTGGT & GCGGAGGCTG & CGAGTGCC \\
\hline NTE I pep & - V.Т..А. & 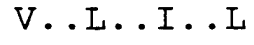 & . I..V..V. & .R. . R . L . . & R..V..P. \\
\hline NTE2 & GGTCACGGCC & GTGCTCATCC & TCCTGGTGGT & GCGGAGGCTG & CGAGTGCC \\
\hline NTE2 pep & .V..T.A. & V..L..I..L & . I..V..V. & .R. . R. I . & $R \ldots V \ldots P$. \\
\hline
\end{tabular}

Figure 6.11 Detection of the initiation codon of the NTE1 CDNA and alternate splice form NTE2 of the 5 ' end of the putative cDNA.

Reading frame 2 of the NTE1 clone gives an unbroken open reading frame starting from base 191, with the first methionine (starting at base 236) as the likely starting codon as it possesses the closest match to the optimum 'Initiator codon (ACCATGG; Kozak, 1986).

The NTE2 sequence diverges from NTE1 9 residues from the putative initiation codon and extends to the 5 ' end of NTE2 in a run of 37 residues unbroken by either initiation or stop codons, indicating NTE2 may represent a form of NTE substantially larger than that encoded by a28. It is unlikely that this is an artefact as the same sequence has been observed in six separate clones(Figure 6.9). 


\section{Chapter 7 Analysis of the NTE cDNA and deduced protein sequence}

\subsection{Introduction}

Chapter 6 describes how the NTE CDNA was eventually cloned using EST data, 5' RACE and conventional hybridisation screening of a CDNA library and also how the open reading frame for NTE was located, this work has now been published in Lush et al, (1998). This chapter attempts to gather all the information relating to NTE (toxicology, biochemistry, protein sequence, peptide mapping and sequence similarities) and integrate it to produce a model of NTE on which future work can be based. Due to the inherent difficulty in predicting protein secondary structure from protein sequence and thus the consequent unreliability of these techniques when used in the absence of supporting data (Argos, 1989), the use of structural prediction programs has been kept to a minimum and, when used, the results treated with caution.

\subsection{Results and Discussion}

7.2.1 The NTE1 clones produced by the 5' RACE contain 11 out of 12 of the NTE peptide sequences.

The DNA sequence from the a28HOL4 contig was translated in all three reading frames. Reading frame +2 contained an ORF of 1342aa in size (Appendix 4) with the initiation codon located 16aa from the start of the ORF (Figure 6.11) to produce a predicted NTE protein sequence of 1327 aa in length. Of the twelve pig NTE sequences (Figures 3.7 and 3.9), eleven found matches within the translated a28HOL4 CDNA sequence (NTE1; Figure 7.1). Only VILP failed to find a match within the sequence, the search was repeated with the available sequence from NTE2 but no further matches were found, indicating that 
VILP was probably a contaminant sequence. The eleven pig NTE peptide sequences were widely dispersed throughout the translated human NTE clone from $A D L T$ at residue 122 to $F D Q I$ at residue 1191. In addition antisera raised to a synthetic peptide of the final $13 \mathrm{C}$-terminal residues in Figure 7.1, reacted with pig NTE on Western blots (P. Glynn, personal communication). It was concluded that the a28HOL4 contig corresponded to one isoform of NTE.

The significance of the alternate splicing at the 5' end of NTE is unclear, the two forms seem to be expressed at roughly equal levels (two NTE1 sequences and only three NTE2 sequences were observed from a more or less random sample of NTE transcripts). Without an initiation codon for NTE2 it is possible that it is responsible for a much larger product (if NTE2 is a truncated cDNA) or much shorter (the next best Kozak sequence is located around base 1048 on the a28HOL4 sequence; see Appendix 4). Two approaches are applicable to determining the extent of alternate splicing in NTE: further 5' RACE using the 5' end sequence from NTE1 to amplify the missing sequence, or, a rather more ambitious approach would be to clone the entire genomic NTE sequence. This task could be approached by designing PCR primers spaced throughout the length of the NTE CDNA sequence and then used to amplify genomic DNA this could be sequenced and assembled to form the genomic clone. The promoter region and 5' end of NTE could be cloned by directedinverse PCR, an inverse PCR library could be constructed using an enzyme known to cut the NTE CDNA near the 5' end, the inverse PCR primers could then be situated between the 5 ' end and the cut site thus they would amplify sequence upstream of the NTE gene. Once the genomic clone had been sequenced computer prediction of the exon-intron boundaries would allow permutations of splicing to be determined and then these predictions could be tested by PCR amplification of cDNA. 


\subsubsection{NTE shares a putative novel esterase domain with proteins from both eukaryotes and prokaryotes.}

BLAST of searches SWISSPROT and EMBL using the deduced NTE peptide sequence have returned 11 protein sequences which have significant similarities with NTE; see Figure 7.2). With the exception of Swisscheese and YOL4, all are hypothetical proteins predicted to exist from genomic DNA sequence. The full database entry for each protein is reproduced in Appendix 2.

The common feature of all these proteins was a domain of about 200 amino acids, which, in NTE, was close to the C-terminal end (Figure 7.3). Interestingly, this region of NTE (residues $920-1120$ ) is precisely that in which the S9B-labelled serine was predicted to reside (See section 5.3). In fact, the data considered in section 5.3 indicate that the catalytic serine lies between NTE residues 955 and 1055; there are 10 serines in this region but particular attention is drawn to Ser966 which is located in the motif GXSXG, characteristic of many serine hydrolases (Brenner, 1988).

A multiple alignment of the regions similar to the NTE C-terminal from the other NTE like proteins was made using GCGs pileup program (Figure 7.4). Evolutionary theory predicts that regions of a protein that are important to its function are better conserved between species than non-critical regions and that the active site of a protein tends to be the best conserved between species as mutation to it is often deleterious to the protein's function (Brenner, 1988). With this in mind GCG program 'plotsimilarity' was used to plot the relative conservation throughout the multiple alignment (Figure 7.5).

On the graph the four regions had a high similarity score and correspond to NTE residues 929-945, 964-970, 1070-1075 and 1083-1087. Peak 964-970 is located on top of the highly conserved putative catalytic serine (Ser966). Given that Ser966 is the only serine located in a minimal serine hydrolase motif (GXSXG) and that the motif is conserved in all the sequences in the alignment, 
Ser966 represents the best candidate for the catalytic serine in NTE. The peak at 929-945 is clearly caused by the near ubiquitous quartet of glycines and the surrounding conserved sequence. Six residues downstream (still within the zone of high conservation) lies a histidine (His945) which is conserved throughout all 11 sequences in the alignment. Since this was the only histidine so extensively conserved, it represents an excellent candidate to be involved in the catalytic triad. The third highest peak between 1083-1087, is located on a region that contains a $100 \%$ conserved aspartate residue in the alignment (Asp1086). Though there are other well conserved aspartates and glutamates in the alignment, Asp1086 is the only one that is flanked by well conserved sequence making is a good candidate to complete the triad.

\subsubsection{Function of the $\mathrm{N}$-terminal domain of NTE}

Among all the proteins with any similarity to NTE only three have an extensive similarity with NTE beyond the 200 residue putative esterase domain: YMF9, sws and YOL4. YMF9 is the least like NTE as it is significantly longer (by 352 residues) and unlike YOL4 and sws; YMF9 has little similarity with the first 500 residues of NTE (see Figure 7.6) so has not been used in this analysis.

YOL4 and sws are better candidates as NTE homologues; both are known to be expressed in vivo (see Figure 7.2) and, as will be discussed later (see Section 7.2.5) mutations to sws produce a neurodegeneration in Drosophila. Therefore, assuming that NTE, YOL4 and sws are homologues which have similar function in all three species regions of similarity between them should delineate zones which are important to the function of the protein.

A gapped-BLAST search of SWISSPROT with NTE returns ambivalent results (gapped-BLAST is a modification of the BLAST algorithm which allows gaps to be introduced into both the database entry and query sequence permitting larger alignments between sequences; Altschul et al, 1997). Aside from strong matches with the putative esterase domain (significance $10^{-21}$ or 
better), there are a number of weak matches with CAMP-regulatory subunits (cRS) which just border on the significant $\left(10^{-7}\right.$ or worse; see Appendix 3$)$. In an attempt to gauge the relevance of the similarities between NTE and the cRS proteins a pair of dotplot graphs of NTE vs YOL4 and NTE vs sws were prepared using GCGs 'compare' and 'dotplot' programs (Figure 7.7). At the bottom of each of the plots the region of similarity between NTE and the 'KAP' proteins (a group of 17 cRS with the highest similarity scores with NTE on the gapped-BLAST search; see Appendix 3). This shows that the cRS similarities between residue 500 and 700 on NTE are located within a functionally significant region of the protein as the peptide sequence is conserved between the NTE homologues.

Direct evidence as to functional importance of this cRS-like domain is provided by Kretzschmar et al (1997) who characterised three of the swisscheese mutations (tabulated below).

$\begin{array}{lll}\text { Phenotype } & \text { Mutation } & \begin{array}{l}\text { Days to } 90 \% \\ \text { Mortality at } 29^{\circ} \mathrm{C}\end{array} \\ \text { wildtype } & \text { na } & 18 \\ \text { sws }^{1} & \text { Ser } 375 \rightarrow \text { stopcodon } & 9 \\ \text { sws }^{4} & \text { Gly648 } \rightarrow \text { Arginine } & 7 \\ \text { sws }^{5} & \text { Gly965 } \rightarrow \text { Aspartate } & 8\end{array}$

From a structural and mechanistic standpoint, there are four interesting points to these data: firstly, sws ${ }^{4}$ is a mutation at residue 648 which corresponds to part of the area of NTE similar to the KAP proteins, this would tend to confirm the presumption of importance for this section of sequence. Secondly sws ${ }^{5}$ is a Glycine to aspartate mutation and effectively adds a charge to the catalytic cleft in much the same way an aged OP does in NTE. Thirdly sws ${ }^{1}$ eliminates $75 \%$ of the protein sequence but has a marginally less severe effect on mortality, relative to the other point mutations. Finally, despite the point mutations (sws ${ }^{4}$ and $s s^{5}$ ) being well separated, they still have a similar gross effect on the insect: this could imply linkage between these two regions of the protein. 
However, no definitive evidence exists and it is difficult to make any useful predictions about this $\mathrm{N}$-terminal domain until more experimental evidence and/or better sequence similarity are available.

\subsubsection{Computer analyses of NTE and YOL4 sequences indicate NTE is a membrane bound protein.}

Transmembrane predictions were made for NTE by three programs: SAPS (Statistical Analysis of Protein Sequences; Brendel et al, 1992), TMpred (Hoffmenn and Soffel, 1993) and SOSUl (Hirokawa et al, 1998). The results are summarised in Figure 7.8. The prediction of transmembrane (TM) domains depends on looking for runs of relatively hydrophobic residues, unfortunately it is not possible to discriminate between these segments and ones that occur in the interior of globular proteins (Creighton,1993), thus TM prediction should always be treated with caution. The TM domain between residues 10-32 was detected by all three programs. The other TM domains predicted by TMpred have much lower scores and two are located near His945 and on Ser966 (Figure 7.1). In the past it has been noted that one of the common features of NTE inhibitors is that they tend to be hydrophobic with small leaving groups (Johnson, 1975b). This could suggest that the environment the catalytic triad is in is also hydrophobic and the hydrophobicity detected by TMpred is attributable to this rather than to any TM domains.

NTE is a membrane associated protein (Section 1.1.5) and the consensus prediction places a TM domain within 10 residues of the $\mathrm{N}$-terminus, the PSORT package (Nakai and Kanehisa, 1992) detects no N-terminal cleavage signal suggesting that the TM domain is present in vivo. Combining the TM predictions and the inferred positions of the catalytic triad it is possible to construct a model of NTE which could be used as a basis for further investigation (Figure 7.9). 
7.2.5 Mutation to the swisscheese (sws) gene can produce a neurodegeneration syndrome or a specific anosmia to aldehydes (olfE).

The swisscheese phenotype was first observed by Heisenberg and Böhl (1987) as part of a screening project for anatomical brain defects in Drosophila. Homozygous insects show reduced lifespan at elevated temperatures $\left(29^{\circ} \mathrm{C}\right.$ gives $90 \%$ mortality after 8.5 days in the average sws mutant, relative to an 18 day lifespan in the wild type). Affected individuals show formation of vacuoles in all regions of the brain; advancing age produces more vacuoles and also apoptotic cell death. Electron microscopy shows neurones enwrapped in multiple layers of membranes derived from nearby glia whereas, in the wild type, glia typically enwrap neurones in one or occasionally two processes (Kretzschmar et al, 1997).

Independent work by Ayyub et al (1990) on the genetics of olfactory behaviour resulted in the characterisation of five olfaction mutants (olfA, olfB, olfC, olfE and olfF) which are all recessive mutations which result in specific reduction (but not elimination) in response to aldehydes (olfA, B, E and F) or acetate esters (olfC). The interesting feature of these mutations is that olfE is produced by the same gene that is mutated in the swisscheese phenotype (Kretzschmar et al, 1997). The other olf genes have been mapped demonstrating that they are separate genes (for example; crossing olfA and olfB gives a near normal phenotype): this may imply that they are part of a signal transduction pathway since disruption to any one of the genes gives a similar phenotype.

Complementation studies of olfE and sws give conflicting results. Hasan showed that germ line transformation of olfE flies with a $14 \mathrm{kbp}$ stretch of genomic DNA containing the wildtype sws gene rescued the olfE phenotype (Hasan, 1990). However, a cross of the olfE/sws ${ }^{1}$ strains show no signs of either the swisscheese or olfE phenotype (Kretzschmar et al, 1997). One possibility is that 
the sws and olfE are separate genes; however, this seems unlikely as the region has been thoroughly searched for transcripts (Paterson and O'Hare, 1991, Kretzschmar et al, 1997). Alternatively it is not unknown for two mutations in a single gene to complement (certain combinations of para and smellblind mutations can complement even though they affect the same gene). Finally it is possible that the sws mutations affect only the $5.4 \mathrm{~kb}$ transcript whereas the olfE mutations affect only the $1.7 \mathrm{~kb}$ transcript (see Figure 7.2): there is a certain amount of evidence for this option since sequencing of the sws5.4 cDNA from one of the olfE insects failed to produce any mutations and it has been suggested that the olfE phenotype is a mutation affecting only the sws 1.7 splice form (Kretzschmar et al, 1997).

\subsubsection{The NTE gene is located on chromosome 19p13.3 near the mapped position of a recessive heritable ataxia.}

The UniGene project is an international collaboration dedicated to genome mapping human EST sequences. The problem is approached using the 3' EST sequence to produce Sequence Tagged Site (STS) genetic markers, then map them relative to two Radiation Hybrid panels and a Yeast Artificial Chromosome library. As of October 1996 more than 16,000 genes have been localised by this method with a clamed reliability of $99 \%$ (Schuler et al, 1996). Over the past few years the STS has become a major genome mapping tool. Essentially an STS is a unique stretch of DNA sequence for which a diagnostic PCR reaction had been developed. By testing for the presence or absence of an STS in a panel of human genome fragments of previously mapped location, it is possible to assign a specific STS to a specific human genome fragment and thus determine its chromosomal location (Schuler et al, 1996).

A search of the UniGene database located ESTs from NTE between markers D19S216 and D19S413: this defines a region between 19.1 and 31.9 centiMorgans (CM) from the top of chromosome 19 in band p13.3. In physical terms this represents an interval of about 13Mbp (Davies and Read 1988); 
however, it may well be a much smaller interval as Nystuen et al (1996) report that the region has an above average recombination rate. Other genes listed as being located in the D19S216-D19S413 interval include the human complement C3 precursor and, ironically, a human EST similar to the Drosophila glutactin precursor (Figure 1.9).

Once a chromosomal location was available, it was then possible to search the On-line Mendelian Inheritance in Man (OMIM, 1997) database for genetic diseases mapped to chromosome 19, looking for diseases which could plausibly be linked with defects in NTE. Of all the genes and diseases linked to chromosome 19, only cerebellar ataxia Cayman type (ATCAY; OMIM Entry Number: 601238; 30/4/1996) survived the most superficial inspection. ATCAY was identified by W. G. Johnson et al. (1978) in a highly inbred population on Grand Cayman Island, and is a rare (only 19 cases have been studyed in Nystuen et al, 1996) recessive disease characterised by marked psychomotor retardation and prominent nonprogressive cerebellar dysfunction including nystagmus, intention tremor, dysarthria, wide-based ataxic gait and hypotonia from early childhood. CT scans performed on two affected individuals showed a marked cerebellar hypoplasia (Nystuen et al, 1996). ATCAY has been mapped by Nystuen et al (1996) to chromosome 19p13.3 in a 9cM interval between markers D19S424 and GATA66B01. The most likely position for the disease is at or near marker D19S216, where the same allele was shared in all 38 chromosomes observed and generated a maximum lod score of $5.98(\theta=0)$. The positions of ATCAY and NTE are summarised in Figure 7.10 indicating that it is possible that ATCAY is caused by a mutation to the NTE gene. However the relationship between ATCAY and NTE is tentative: the mutated gene could easily be the human homologue of Drosophila glutactin (see Section 1.1.6) which is also located in that interval.

To determine if ATCAY is caused by mutation to the NTE gene first it would be necessary to map the NTE gene to a much finer level than has been achieved by the UniGene project. This could be addressed by obtaining YACs 
covering that area of chromosome 19 from the Human Genome Mapping Project resource centre, and determining by hybridisation which YACs NTE is located on, the YACs could be assembled into a contig by chromosome walking and the positions of D19S216, GATA66B01, and D19S413 could be determined by hybridisation. If NTE is located near D19S216 (and hence the ATCAY gene) it would be possible to collaborate with Nystuen et al (1996), assay NTE activity in affected individuals and ultimately clone and sequence the NTE CDNA (as NTE is expressed in blood lymphocytes, a relatively assessable source of RNA for use in CDNA synthesis and cloning). Ultimately it seems unlikely that ATCAY mutation will yield critical information about NTE, however currently it would be the only human model of malfunction in NTE besides that caused by OP and may yield some insight. 


\subsection{Overview of predictions}

The main findings of this chapter are that:-

- The cDNA clones discussed in Chapter 6 encode NTE and furthermore at least two alternately spliced versions of the NTE mRNAs exist.

- NTE is a novel serine esterase protein which has homologues in a wide range of species and the probable locations of the catalytic triad are His945, Ser966 and Asp1086.

- The esterase domain is apparently quite compact, possibly encoded by as few as 200 amino acids and similar sequences exist in a wide variety of organisms.

- NTE has one reasonably credible transmembrane domain located at the $\mathrm{N}$ terminal.

- The Drosophila homologue of NTE (swisscheese) has a number of mutations which have more or less the same effect on survival and suggest close interactions between separate regions of the protein: all sws mutants show an age-dependant neurodegeneration.

- Mutations in NTE may cause a neurodegenerative disease; ESTs encoding NTE have been mapped to a region of chromosome 19p13.3 close to the positions of a recessive heritable ataxia. 
These are a number of questions still unanswered:-

- What is the membrane topology of NTE and what organelles is it associated with?

- What are the biological functions of the various domains of the NTE protein?

- What is the biological role of NTE and what is its role in the development of OPIDP?

\subsection{Future work}

The first priority in continuing the research project into NTE is to fully sequence all the variants of NTE produced by the 5' RACE. Once this is complete, besides maintaining surveillance on the genetic databases with regular BLAST searches, the questions posed at the end of the previous section could be addressed thus.

7.4.1 What is the membrane topology of NTE and what organelles is it associated with?

Since NTE was cloned subsequent work has succeeded in transiently expressing catalytically active (D16) NTE in COS cells (Jane Atkins, personal communication). This work could provide the basis to test the structural predictions made in Figure 7.9 (ie that NTE has an N-terminal TM domain and almost all the protein is located on one side of the membrane). One method that has been used to study membrane topology was proteolytic digestion of microsomes which had been incubated in the presence of a in vitro transcriptiontranslation system (Olender and Simoni, 1992). NTE expressed in such a system should be processed by the microsomes and, when the proteinase is added, the NTE should either be totally destroyed (if it has a cytosolic Cterminal) or left essentially untouched (if the $\mathrm{N}$-terminal is cytosolic). If NTE has 
more than one transmembrane domain a section of NTE should remain untouched as the cytosolic domains are digested by the proteinase.

For a detailed localisation of NTE to specific organelles the current generation of antisera raised to synthetic peptides is of limited use as their binding is sensitive to aldehyde fixation; this severely limits the microscopic resolution possible (though confocal microscopy is possible as this can be performed on unfixed or acetone-treated cells). However, a more robust polyclonal antisera could be raised to bacterially-expressed NTE domain to allow more extreme fixation procedures required for immunoelectron microscopy work, which would be able to resolve NTE's location and orientation in the cell.

\subsubsection{What are the biological functions of the various domains of the NTE protein?}

Recombinant forms of NTE offer one avenue to probing the functions of NTE; one useful modification would be a version of NTE starting at residue 34 , omitting the transmembrane domain and incorporating a His tag to allow easy purification of the native enzyme. Assuming the single TM domain model is true, this should produce a more soluble form of NTE (presently NTE is soluble only in the presence of detergent; Davis and Richardson, 1987) which would be easier to study.

The suggestion that NTE binds cyclic nucleotides (Section 7.2.3) could then be tested by incubating purified NTE with radiolabelled CAMP or CGMP and assaying for binding. Should binding be detected it could be further localised by expressing portions of NTE in a suitable eukaryotic expression vector (ie one that has start and stop codons flanking the polycloning site as well as some form of purification system such as a His tag). This technology could be used to isolate and analyse the function of any part of NTE. Initially the main region of interest would be the 200 residue esterase domain (see Sections 7.2.2). If it could be 
demonstrated that just the esterase domain was catalytically active then structural studies could commence using NMR and X-ray crystallography.

An 'esterase only' version of NTE could also be used to test the other prediction made in Figure 7.9, that the catalytic triad is composed of His945Ser966-Asp 1086 by mutating each of the three and determining the effect on catalysis, although the results would not be definitive as even if unrelated to the catalytic triad, mutation of these residues could have a severe effect on the protein as they are located in highly conserved regions. Only 3D structure (from NMR or X-ray crystallography) could ultimately resolve the residues comprising the catalytic triad.

The other question which could be addressed by protein expression is what other proteins does NTE interact with. There are two systems suitable for the task: phage display, in which an M13 phage cDNA library has been engineered to express the cDNAs as part of their coat protein; phage that do not bind immobilised NTE can be washed away, those that do bind can be eluted and propagated for further investigation (Dang et al, 1991). The other relevant system is the yeast two-hybrid system (Iwabuchi et al, 1993), in which part or all of the NTE protein would be fused with a DNA binding protein; interactions between NTE and a library of proteins activate the DNA binding protein (via an activator protein fused to the library proteins). The protein complex then activates a reporter gene, the activity of which is used to isolate possible positive interactions.

Of the two systems the yeast two hybrid seems the most likely to work as all the protein is expressed in a eukaryotic environment processing of the protein is more likely to be in the native form, additionally Clontech produce a mammalian version of the two-hybrid assay which would be useful if there were problems expressing NTE in a yeast host. 


\subsubsection{What is the biological role of NTE and what is its role in the development of OPIDP?}

The NTE like genes in Drosophila (sws) may provide a useful model of OPIDP. When attempting to draw parallels between the swisscheese phenotype and OPIDP; the principal difference to consider (besides species) is that the sws $^{5}$ mutation is effectively a constitutive ageing of the gene throughout the insect's development, whereas OPIDP is caused by a single transient event in an adult. To reconcile the two modes of action, two approaches are possible, production of an NTE knockout mouse would produce a constitutive loss of NTE activity more equivalent to sws ${ }^{1}$. The other approach would be to collaborate with Kretzschmar et al (1997), in determining if Drosophila sws has any NTE like activities; such as binding of known neuropathic OPs (preferably ones not developed as insecticides). If a suitable OP can be found then it would be possible to start to dissect the role of sws in development by inhibiting sws (either transiently or constitutively) at various stages of the insect's life cycle to determine if there is any significant variation in the phenotype.

Similar work is possible with YOL4 although the background on it is less well developed (though Hope et al, (1996) have announced a 'C. elegans expression pattern database' which may one day include YOL4 as part of the project involves reporter gene studies on the promoters of hypothetical proteins). Nevertheless, both Drosophila and C. elegans are easily genetically manipulated (due to their short lifecycle) and thus could be used to screen putative NTE binding proteins (produced by the yeast two hybrid system; Section 7.4.2) by knocking out the insect or worm homologue of the binding protein. 


\subsection{Conclusion}

Over the duration of this project considerable advances have been made in the understanding of NTE. Not only has the CDNA been cloned, permitting experiments that directly address the initiation of OP induced-neuropathy at a molecular level, but also NTE has been established to be a novel serine esterase and with a homologue in Drosophila which has a key role in neuronalglial cell interactions during development. With these advances it is possible to start to investigate the unknown events that still lie between ageing of NTE and neuropathy 1 to 3 weeks later. Or to put it another way, the smoking gun of OPIDP has been found, now all that remains to do is find out what the bullet looks like, where it went and who it hit. 
1 MEAPLQTGMV LGVMIGAGVA VVVTAVLILL VVRRLRVPKT PAPDGPRYRF

51 RKRDKVLFYG RKIMRKVSQS TSSLVDTSVS ATSRPRMRKK LKMLNIAKKI

101 LRIQKETPTL QRKEPPPAVL EADLTEGDLA NSHLPSEVLY MLKNVRVLGH ADLTEGDLA NSHLPPEVL

151 FEKPLFLELC RHMVFQRLGQ GDYVFRPGQP DASIYVVQDG LLELCLPGPD

201 GKECVVKEVV PGDSVNSLLS IIDVITGHOH PORTVSARAA RDSTVLRLPV VV PGDSVNSILS IIDVITGXQA PQ

251 EAFSAVFTKY PESLVRVVQI IMVRLQRVTF LALHNYLGLT NELFSHEIQP

301 LRLFPSPGLP TRTSPVRGSK RMVSTSATDE PRETPGRPPD PTGAPLPGPT IRLFPSPGLP ARTSPVR

351 GDPVKPTSLE TPSAPLLSRC VSMPGDISGL QGGPRSDFDM AYERGRISVS

401 LQEEASGGSL AAPARTPTQE PREQPAGACE YSYCEDESAT GGCPFGPYQG

451 RQTSSIFEAA KQELAKLMRI EDPSLLNSRV LLHHAKAGTI IARQGDQDVS

501 LHFVLWGCLH VYQRMIDKAE DVCLFVAQPG ELVGQLAVLT GEPLIFTLRA

551 QRDCTELRIS KSDFYEIMRA QPSVVLSAAH TVAARMSPFV RQMDFAIDWT

601 AVEAGRALYR QGDRSDCTYI VLNGRLRSVI QRGSGKKELV GEYGRGDLIG

651 VVEALTRQPR ATTVHAVRDT ELAKLPEGTI GHIKRRYPQV VTRLIHLLSQ

IAAKLPEGTL GHIWRRYPQV V

$\downarrow$ start of HOL 4.2 sequence

701 KILGNLQQLQ GPEPAGSGLG VPPHSELTNP ASNLATVAIL PVCAEVPMVA LTNP ASNLATVAVI

751 FTLELQHALQ AIGPTLLLNS DIIRARLGAS ALDSIQEFRL SGWLAQQEDA

801 HRIVLYQTDA SLTPWTVRCL RQADCILIVG LGDQEPTLGQ LEQMLENTAV

851 RALKQLVLLH REEGAGPTRT VEWLNMRSWC SGHLHLRCPR RLFSRRSPAK

901 LHELYEKVFS RRADRHSDFS RLARVLTGNT IALVLGGGGA RGCSHIGVLK

951 ALEEAGVPVD LVGGTSIGSF IGALYAEERS ASRTKQRARE WAKSMTSVLE XGVPVA IVGP

1001 PVLDLTYPVT SMFTGSAFNR SIHRVFQDKQ IEDLWLPYFN VTTDITASAM DLGLPYFN VVTDI

1051 RVHKDGSLWR YVRASMTLSG YLPPLCDPKD GHLLMDGGYI NNLPADIARS D GHLLTDGGYI NNLP

1101 MGAKTVIAID VGSODETDLS TYGDSLSGWW LLWKRLNPWA DKVKVPDMAE

1151 IQSRLAYVSC VRQLEVVKSS SYCEYIRPPI DCFTTMDEGK EDQIYDVGYO VVRSS SXDGXIRXXI DYFK FDQIYDVGYQ

1201 YGRAVEGGWS RGNVIEKMLT DRRSTDLNES RRADVLAFPS SGFTDLAEIV YGK

1251 SRIEPPTSYV SDGCADGEES DCLTEYEEDA GPDCSRDEGG SPEGASPSTA

1301 SEMEEEKSIL RQRRCLPQEP PGSATDA

Figure 7.1 Positions of the known pig NTE peptide sequences on the translated sequence of human NTE CDNA a28HOL4.

Identities between NTE peptides and translation are shown in bold. Transmembrane domains predicted by one program are shown single underlined, transmembrane domains predicted by three programs are shown double underlined (see Figure 7.8). 


\begin{tabular}{|c|c|c|c|}
\hline Protein Name & $\frac{\text { Accession }}{\text { Number }}$ & Length (aa) & Origin \\
\hline YOL4_CAEEL & sw:Q02331 & 1351 & C. elegans \\
\hline Swisscheese & em: Z97187 & 1425 & Drosophila \\
\hline YCHK_ECOLI & sw:P37053 & 314 & E. coli \\
\hline YCHK_SHIFL & sw:P37054 & 200 (fragment) & Shigella flexneri \\
\hline YLBK & em: Z98682 & 260 & Bacillus subtilis \\
\hline Mtcy20b11 & tr:005884 & 1048 & Mycobacterium tuberculosis \\
\hline YOA3_MYCTU & sw:Q50733 & 583 & M. tuberculosis \\
\hline YVL7_CAEEL & sw:Q21534 & 880 & C. elegans \\
\hline YMF9_YEAST & sw:Q04958 & 1679 & Saccharomyces cerevisiae \\
\hline YK69_YEAST & sw:P36165 & 910 & S. cerevisiae \\
\hline Q12 & pir:Q12043 & 749 & S. cerevisiae \\
\hline
\end{tabular}

Figure 7.2 Summary of the hypothetical proteins with similarity to NTE.

Where possible the SWISSPROT name is used; the name is abbreviated in the main text by omitting species identifier. When the protein is not from SWISSPROT the most meaningful name was selected from the database entry (all of which are reproduced in full in Appendix 2).

Of these sequences two are known to be expressed in vivo: YOL4 has 17 matching $C$. elegans EST sequences registered in EMBL; swisscheese (sws) has been shown by Northern blotting to exist as $5.4 \mathrm{~kb}$ and $1.7 \mathrm{~kb}$ transcripts (sws 5.4 and sws 1.7). Sws5.4 has been cloned and sequenced and is expressed in Drosophila heads and bodies at all stages of development; in situ hybridisation shows, in brain, it is localised in neurons but not glia. Sws1.7 has not been cloned but is known to be derived from the 5 ' end of sws5.4 and is expressed weakly in Drosophila heads (Hasan, 1990 and Kretzschmar et al, 1997).

The eleven sequences listed above do not comprise an exhaustive list of all the known NTE-like sequences. BLAST searches of the pre-release DNA sequence data at TIGR (The Institute for Genomic Research, personal communication) shows that the genomes of Neisseria meningitidis, Vibrio cholerae, Neisseria gonorrhoeae, Treponema pallidum, Deinococcus radioduran and Thermotoga maritima all contain NTE homologies. All these organisms are important pathogens and it is just possible that one of the non-neuropathic NTE inhibitors could have an antibiotic effect on them. 


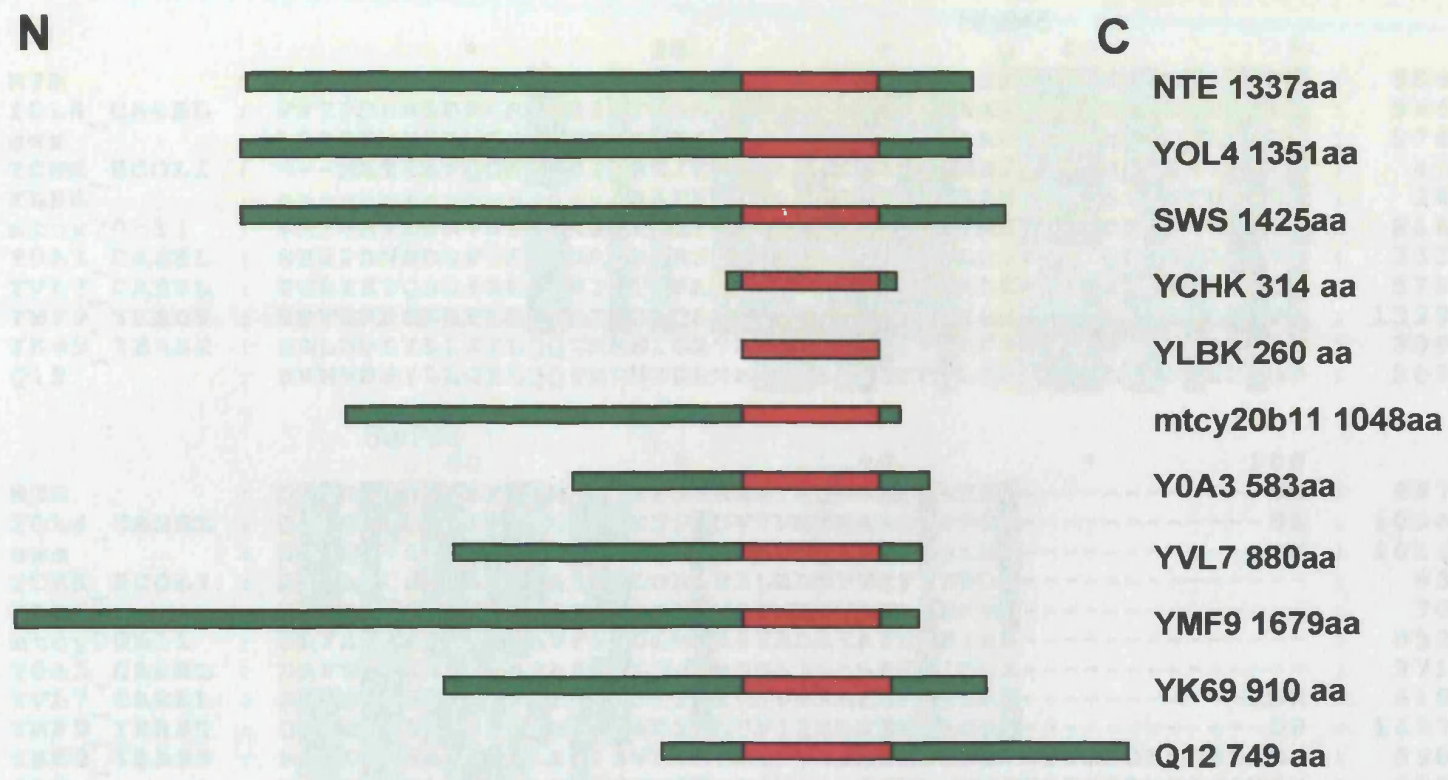

Figure 7.3 Comparison of NTE like proteins.

The putative NTE esterase domain is marked in red. 
NTE

YOI 4_CAEEL sws

YCHK ECOLI YLBK

m tcy $20 b_{11}$

YOA 3 CAEEL

YVL $7^{-}$CAEEL

YME 9 YEAST

YK $69^{-}$YEAST

Q12

NTE

YOL 4_CAEEI

SW

YCHR ECOLI

Y LBK

m tcy $20 b_{11}$

YOA 3 CAEEI

YVI $7^{-} \mathrm{CAEEI}$

YME9- YEAST

YK $69^{-}$YEAST

Q12

NTE

YOL 4 _ CAEEL

SwS

YCHK_ECOLI

$Y \mathrm{LBK}$

m tcy $20 \mathrm{~b} 11$

YOA 3 CAEEI

YVL 7 CAEEL

YME 9 YEAST

YK69 YEAST

Q12

NTE

YOL 4_CAEEL

sws

YCHK_ECOLI

Y LBK

mtcy $20 \mathrm{bl} 1$

YOA 3 CAEEL

YVL $7^{-}$CAEEL

YME $9^{-}$YEAST

YK $69^{-}$YEAST

Q12

NTE

YOL 4 CAEEL

SWS

YCHK ECOLI

Y LBK

mtcy 20 b1 1

YOA3 CAEEI

YVL $7^{-}$CAEEL

YMF $9^{-}$YEAST

YK69-YEAST Q12

His 945

\author{
20
}

SRRADRHSDFSRLARVITGNTIALVLGGGGARGC SHIGVLKALENAGVPV : 959

FWT PDRRSDFSRLARI LTGNAIGLVLGGGGAR GAAHVGVLRALREEGIPV : 966 LSEPNMHSDFSRLARWLTGNS IGLVLGGGGAI.GAAHIGMLKAIQEAGIPV : 978 -- MATIAFOGNLAGIMRKIKIGLALGSGAAI:GWSHIGVINALKKVGIEI : 47 作 VHYRR I IENVR PLAARIAGRS IGLVLGGG GARGFAHLGVLDELERVGVTI : 816 SH H PAN SD GFGALARR I AGRSLGLVMAGG GAKGLAHFGVY QELTEAGVVI : $\quad 335$ YGE ISYQSD FSRLARI LTGNAIGIVFGGG SAI:GAAHAGALRALIEKRVQI : 579 TPVHRH KND FLRLARI L SQA I GLVLGGG GAKGI SHLGVIQAIEEQGIPV : 1399 SDLDDSYLLGILOOTRRNIGRTALVLSGG GTFGLFHI GVLGTLFELDLL P : SNMNDHYLLGILQQTRRNIGRTALVLSGGSTFGLFHIGVLAALFISDLMP : 209

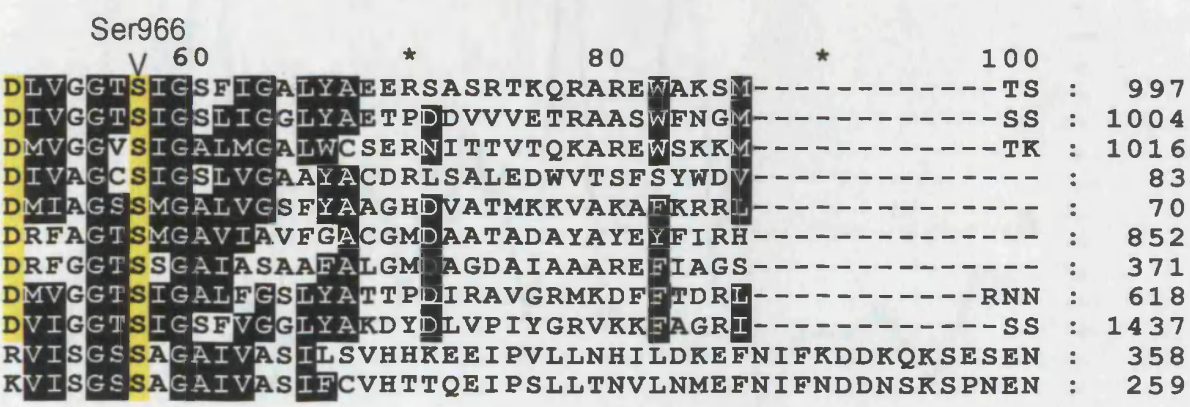

VLEPVTDLTYPVTSMFTESAFNRSTHRVFQDKQIEDLUTLPYFNVTTDITA LWRK LDLTYAH SAMFT TAQFNFSTIRDLFERLIEDLWI SYFCISTDIST : 1054 WFLOILDLTYPI TSMFSGREFNKTTHDTFGDVSIFDLWI PYFTLTTDITA : 1066 - - LRIMDLSWQRGGLLREERVFNQYREIMPETEIENCSRRFAAVATNLST : 131 - - YADY TVPKLGFLKGDRVRQLVHAYTFGKPIEELQIPIGIVACDLQT : 116 - -NPLSDYAFPVRGLVRGRRTLTLLEAAFGDRLVEELPKEFRCVSVDLLA : 900 - -DPLGDYTIPISALTRGGRVDRLVOGFFGNTLIEHLPRGFFSVSADMIT : $\quad 419$ ILDVVRDI YYGILTGHRFNLC QRMLNLVNIEDCWVSFFCITTDLTS : $\quad 668$ IWRMLTDLTWPVTSYTTGHEFNRG WRTFGT TRIEDEWIQYYCNSTNITD: 1487 LLIKTSRE-FKNGTWFDNKHLVNTMIE FLGDLTFREAYNRTGKT-LNITV : 406 LLIKISRF-CQNGTWFNNQPLINTMLSFLGNI,TFREAYNRTGKI-INITV : 307

160

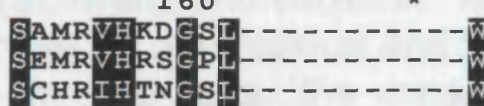

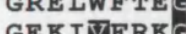

RRPVVHRR GS

GDQI I HRRGS

SSMRIHRN GI

SPASTFROPRT

SPASLFEQPRLLNNLTAPNVLI WSAV
SPASIYEQPKLLNNLTAPNVLIWSAV
CHRIHTNGS

180

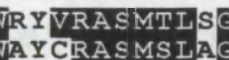

WRYVRSSMSLSG

- HLAIRASCS I

DVIGCS LRL

GAVRAS IS IPG I

TPI

LPPLCDPODGH - -

LCD PRDGH---

QRLDGR - - - -

OVYNGR - - - -

VHNGEO-- - - -

CDPQDGH---

SPI Y

240

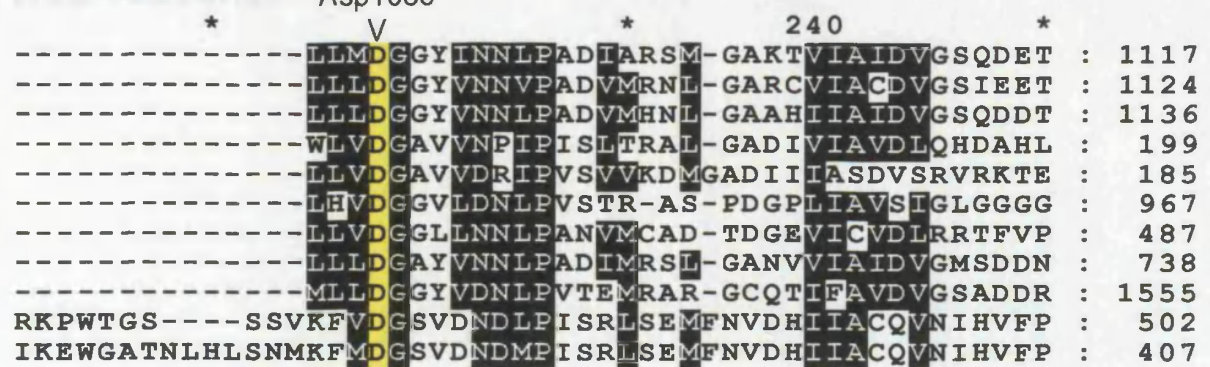

Figure 7.4 Multiple alignment of the putative esterase domain of proteins in Figure 7.3.

The number at the right side of the alignment indicates the residue number for the appropriate sequence. 


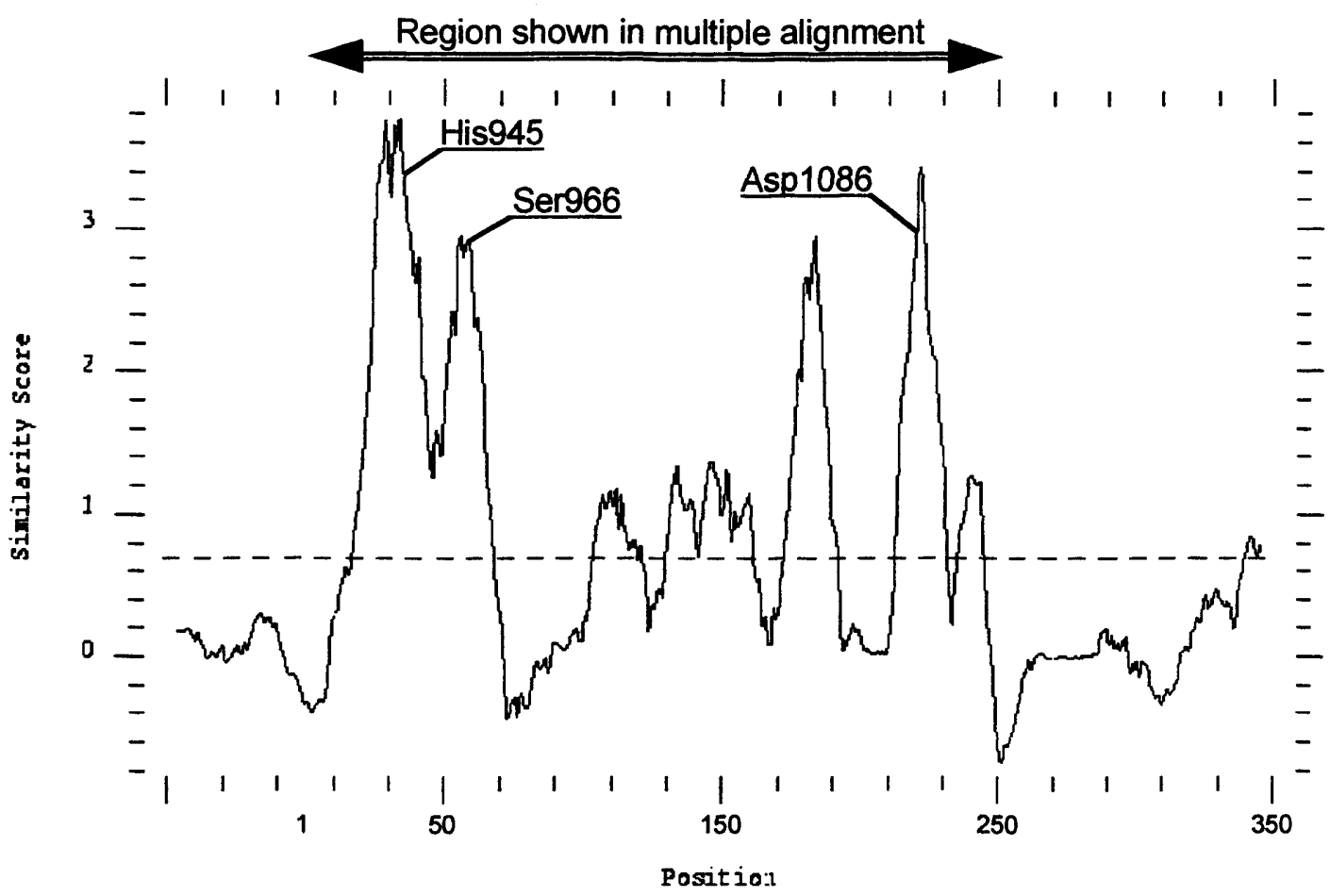

Figure 7.5 Similarity plot of data in Figure 7.4

The GCG program plotsimilarity was used to produce this graph. The program scores a 'window' of 10 residues assessing the overall similarity of all the columns of residues to determine a score based on the Dayhoff amino acid similarity matrix (Schwartz and Dayhoff, 1979). The score is plotted on a graph, the window is moved on one residue and the process is repeated. The higher the point on the graph, the better the conservation between sequences is in that region.

The graph represents the part of a multiple alignment in which all the sequences show significant similarity and has been renumbered to match the alignment shown in Figure 7.4. The dashed line represents the average peak height. The positions of the His, Ser and Asp are numbered relative to the start of the ungapped NTE sequence. 


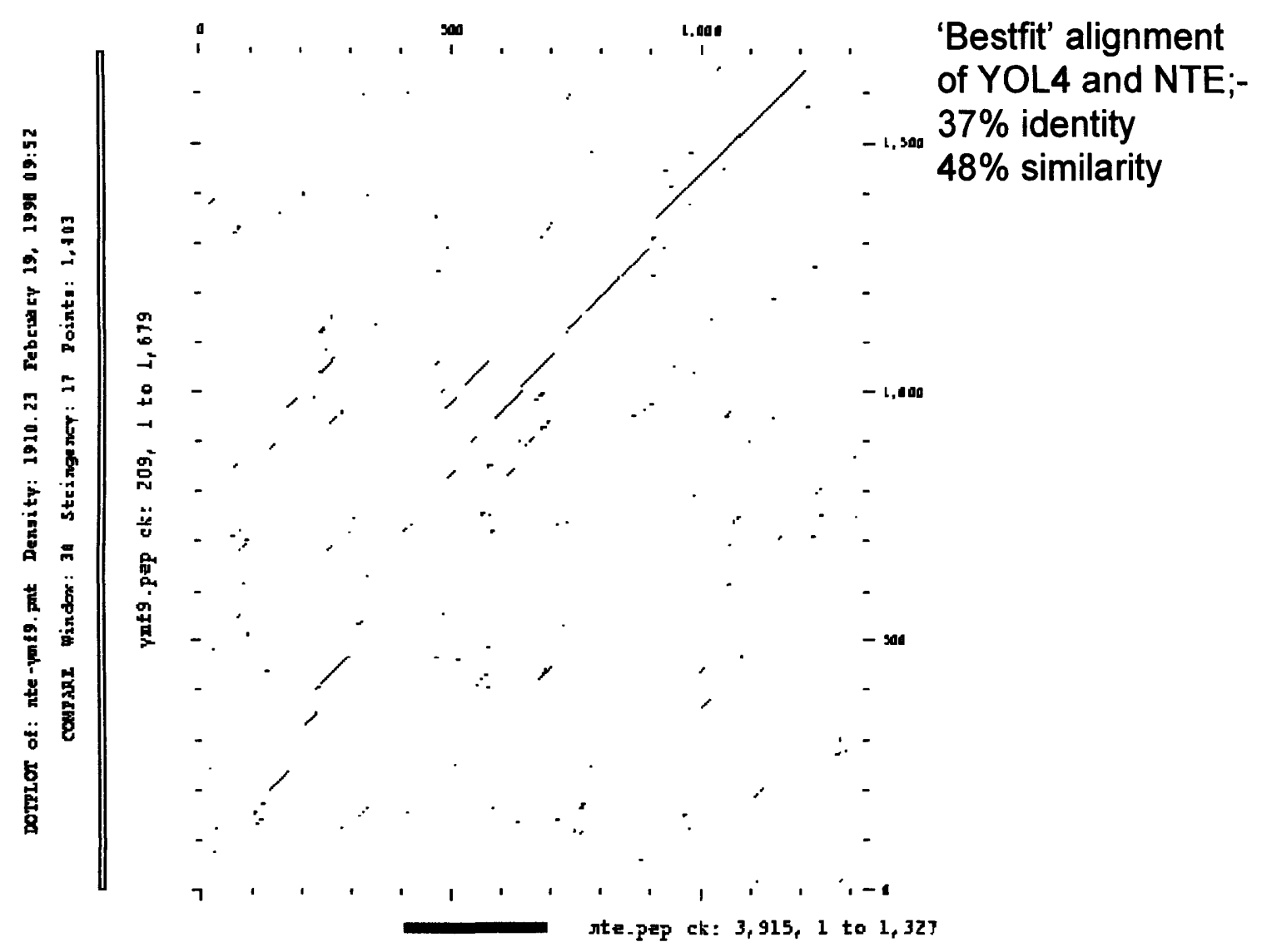

Figure 7.6 Dotplot of YMF9 (vertical) vs NTE (horizontal).

The red bar indicates the region of NTE sequence similar to the KAP group of proteins returned by the gapped-BLAST search. Window size 30 residues, stringency 17 

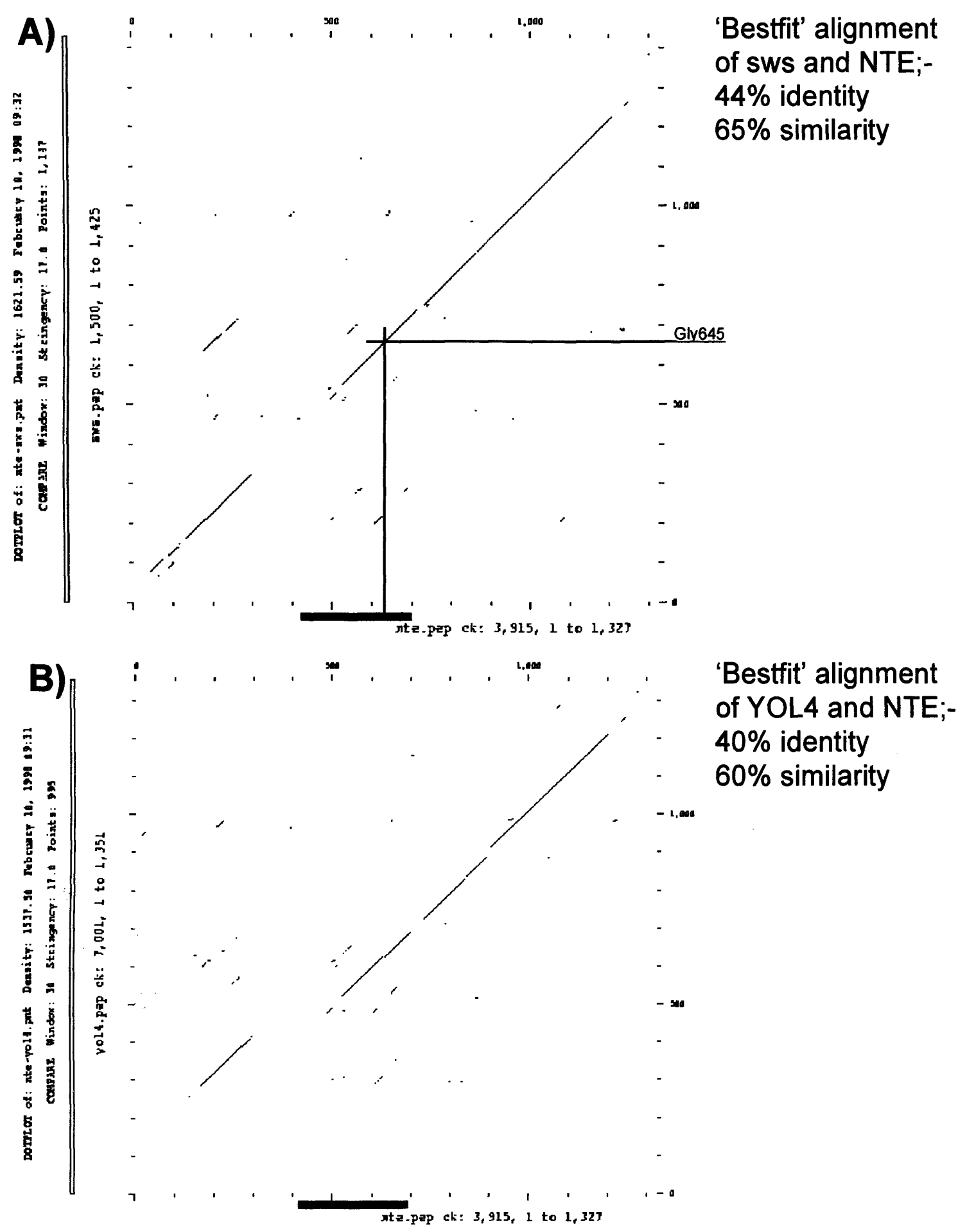

Figure 7.7 Dotplot of NTE (horizontal) vs sws (A) and YOL4 (B).

The red bar indicates the region of NTE sequence similar to the KAP group of proteins returned by the gapped-BLAST search. The comparison was done using GCGs 'compare' program with a comparison window of 30 residues at a stringency of 17 identities. 


\begin{tabular}{|c|c|c|}
\hline Residue No. & Predicted transmembrane region & Prediction program \\
\hline 8 to 32 & GMVLGVMIGAGVAWVTAVLILLW & SAPS \\
\hline 10 to 32 & VLGVMIGAGVAWVTAVLILLW & sosul \\
\hline 11 to 32 & LGVMIGAGVAWWTAVLILLW & TMpred (score 2375) \\
\hline 734 to 753 & LATVAILPVCAEVPMVAFTL & TMpred (score 958) \\
\hline 925 to 943 & VLTGNTIALVLGGGGARGC & TMpred (score 1087) \\
\hline 956 to 976 & GVPVDLVGGTSIGSFIGALYA & TMpred (score 1276) \\
\hline
\end{tabular}

Figure 7.8 Transmembrane predictions for NTE.

All three programs detect a possible transmembrane domain located close to the N-terminal of NTE, the other three hits registered by TMpred seem to be less convincing, as the score is lower (though still significant at $>500$ ) and the two highest are located near or over the putative catalytic domain (see Figure 7.1). 


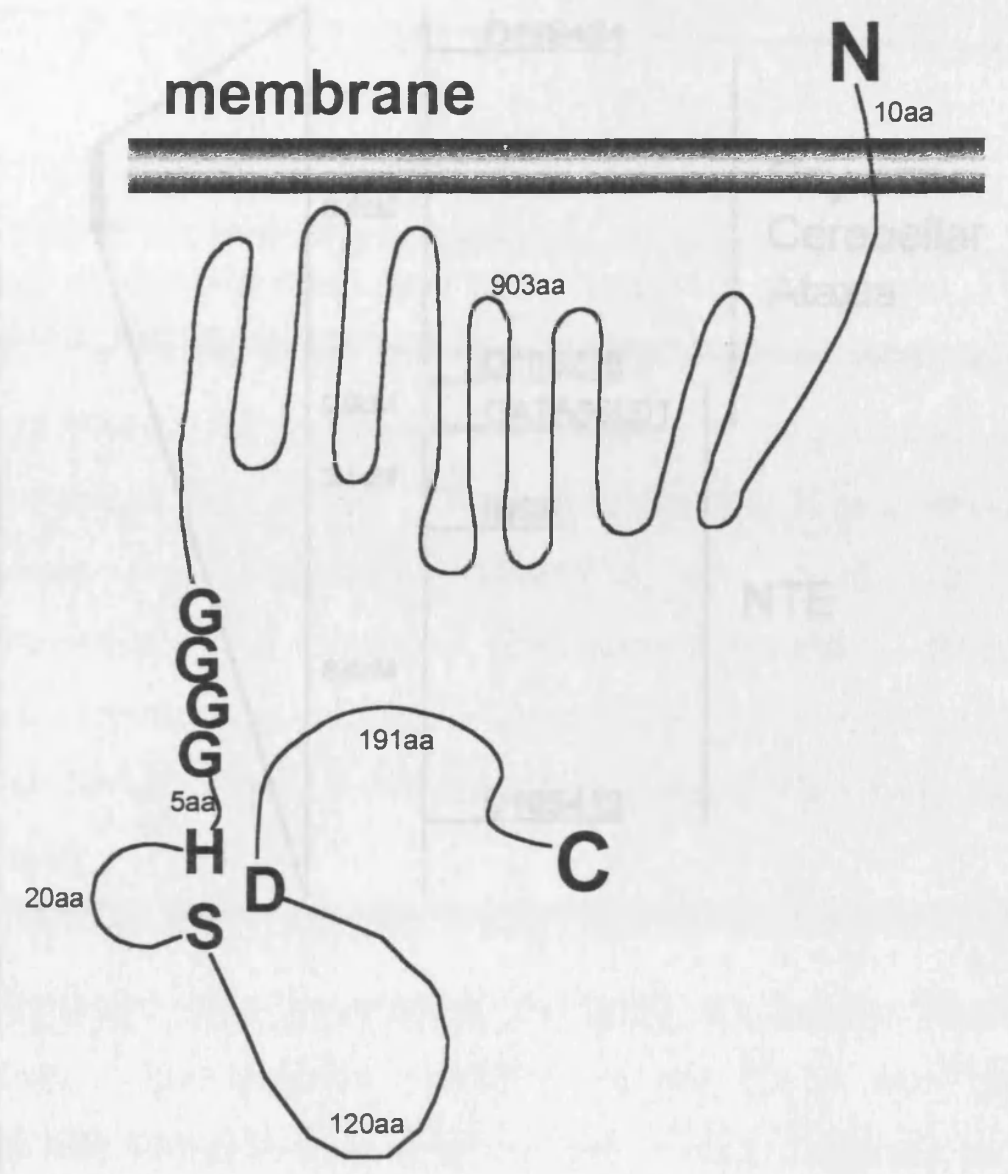

Figure 7.9 Putative secondary structure of NTE (not to scale).

The TM prediction suggests the transmembrane domain is at the $\mathrm{N}$ terminus with a short span of protein sequence emerging from the membrane. To be functional the His, Ser and Asp residues of the catalytic triad must be in close proximity. 


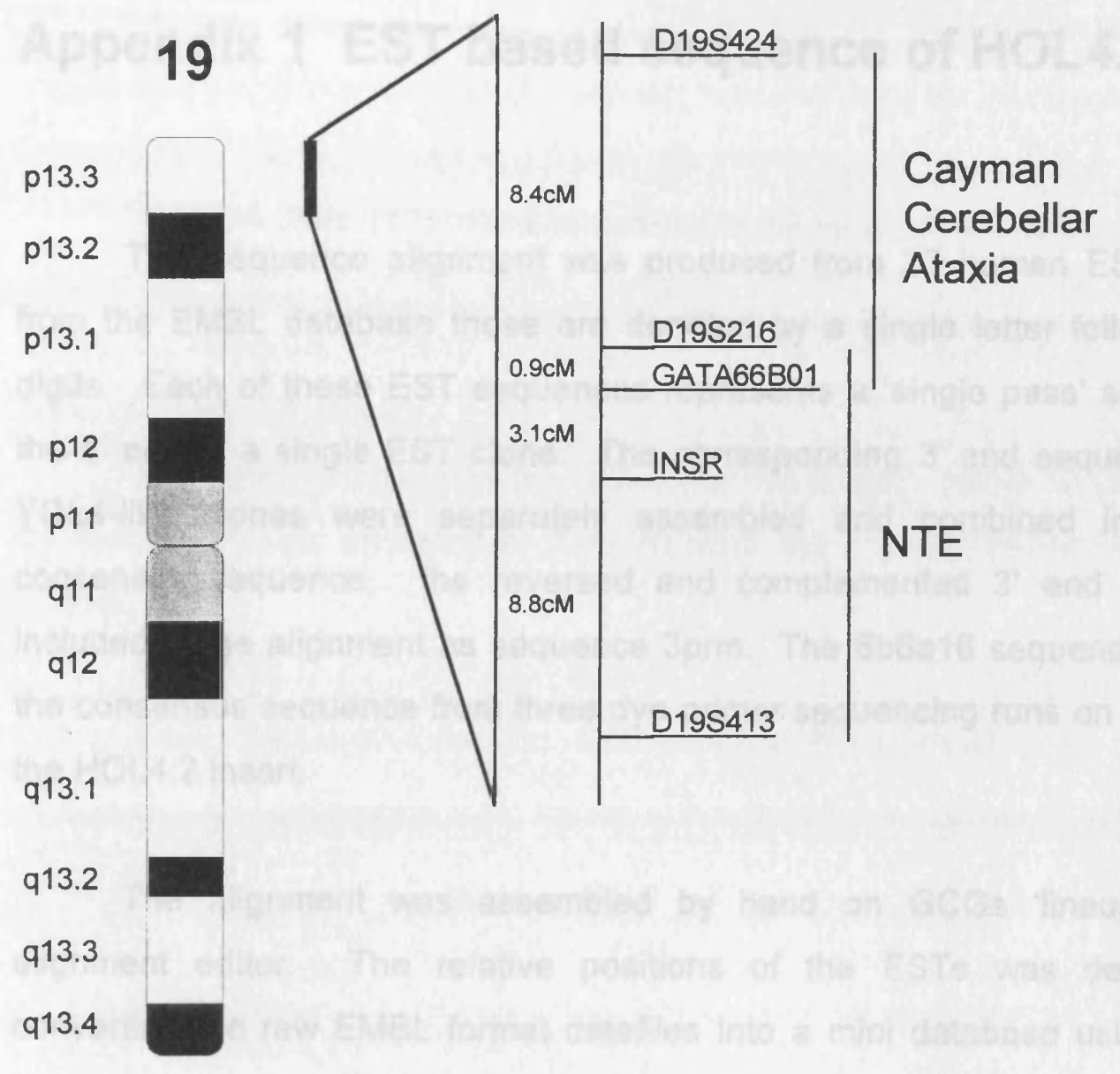

Figure 7.10 Cytogenetic map of Chromosome 19 and genetic map of the ATCAY/NTE location. 


\section{Appendix 1 EST based sequence of HOL4.2}

This sequence alignment was produced from 23 human ESTs retrieved from the EMBL database these are denoted by a single letter followed by five digits. Each of these EST sequences represents a 'single pass' sequencing of the 5' end of a single EST clone. The corresponding 3 ' end sequences for the YOL4-like clones were separately assembled and combined into a single consensus sequence, the reversed and complemented $3^{\prime}$ end sequence is included in the alignment as sequence $3 \mathrm{prm}$. The $8 \mathrm{~b} 6 \mathrm{a} 16$ sequence represents the consensus sequence from three dye primer sequencing runs on the 5 ' end of the HOL4.2 insert.

The alignment was assembled by hand on GCGs 'lineup' sequence alignment editor. The relative positions of the ESTs was determined by converting the raw EMBL format datafiles into a mini database using the GCG 'toblast' program, then performing BLAST searches of that database using individual EST sequences. BLAST was able to detect any of overlap between the search sequence and any other EST. These data could then be assembled into the alignment below running from just beyond the LTNP sequence to the polyadenylation signal (shown in red) and poly-A tail.

The consensus sequence for the alignment (cons) was then translated in all three forward reading frames and the DNA sequence were also used to search SWISSPROT using BLAST. The correct reading frame for any given section of sequence was determined by comparison to the known NTE peptide sequences LTNP, XGVP, DLGL, DGHL, WKS and FDQI. Regions in the alignment where these sequences match have an extra line 'nte-seq'. The YOL4 peptide sequence was also used to fix the reading frame where BLAST showed a significant similarity between the two sequences. For reasons of clarity these matches are not shown in this alignment. 
Regions where the reading frame was fixed by comparison to homologous sequences are shown double underlined; regions were the sequence was merely inferred from the known reading frame are shown single underlined.

The first 66bp of $\mathrm{H} 10469$ and the first 19 bp of T10299 do not match the consensus, this may represent isoforms of NTE but it is more likely they represent immature mRNAs the reagions of mismatch with the consensus have been blocked out with Ns.

This figure is based on the GCG .msf file format. However to save space, all the blank lines except the ones flanking the current sequence have been deleted.

List of EMBL/Genbank accession numbers used in alignment.

$\begin{array}{ll}\text { t09456 } & t 66368 \\ r 87932 & t 10299 \\ r 89699 & h 58564 \\ \text { h05032 } & r 51195 \\ r 56734 & r 15042 \\ r 13907 & h 61615 \\ \text { t23706 } & r 51470 \\ \text { r12067 } & r 45802 \\ r 25505 & r 54769 \\ \text { h09837 } & r 48493 \\ \text { h10469 } & r 47780 \\ r 17727 & \text { m78841 }\end{array}$


$8 \mathrm{~b} 6 \mathrm{a} 16$

t09456

cons

frame 1

frame2

frame 3

$8 \mathrm{~b} 6 \mathrm{a} 16$

t09456

cons

frame 1

frame2

frame 3

NTE-pep

$8 \mathrm{~b} 6 \mathrm{a} 16$

t09456

r87932

cons

frame 1

frame2

frame 3

NTE-pep

$8 \mathrm{~b} 6 \mathrm{a} 16$

t09456

r87932

cons

frame 1

frame2

frame 3

$8 \mathrm{~b} 6 \mathrm{a} 16$

t09456

r87932

r89699

h05032

cons

frame 1

frame2

frame 3

$8 \mathrm{~b} 6 \mathrm{a} 16$ t09456 r87932

r89699

h05032

cons

frame 1

frame2

frame 3

AATTTGCAGC AGCTGCAAGG ACCCTTCCCA GGCTCTGGGT TGGGTGTGCC AATTTGCAGC AGCTGCAAGG ACCCTTCCCA GGCTCTGGGT TGGGTGTGCC ..F..A..A. .A..A..R.. T..I..P..R ..L..W..V. .G..C..A.

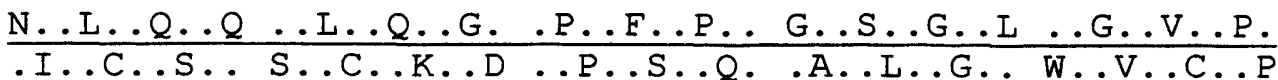

51

CCCACACTCG GAACTCACCA ACCCAGCCAG CAACCTGGCA ACTGTGGCAA

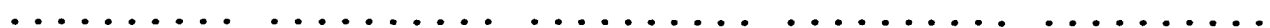
CCCACACTCG GAACTCACCA ACCCAGCCAG CAACCTGGCA ACTGTGGCAA P..T..L..G ..T..H..Q. .P..S.Q.. Q..P..G..N ..C..G..N. .P..H..S..E..L..T..N ..P.A..S..N..L..A.. T..V..A..I

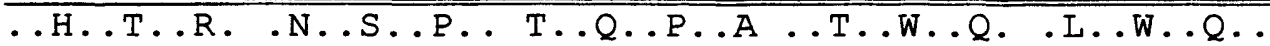
$\ldots \ldots \ldots \ldots$...L.T.N …A..S. .N..L.A. T.V..A.V

101

TCCTGCCTGT GTGTGCTGAG GTCCCCATGG TGGCCTTCAC GCTGGAGCTG ............ CTGAG GTCCCCATGG TGGCCTTCAC GCTGGAGCTG

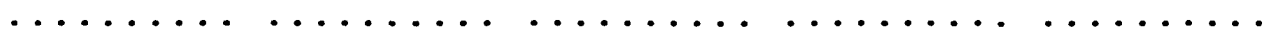
TCCTGCCTGT GTGTGCTGAG GTCCCCATGG TGGCCTTCAC GCTGGAGCTG .P..A..C..V..C..*..G ..P..H..G. .G..I..H.. A..G..A..A .L..P..V. .C..A..E..V..P..M.VV ..A..E.T. .L..E..I.

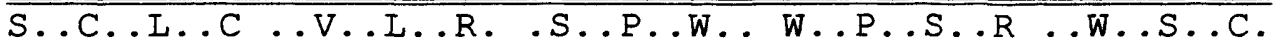

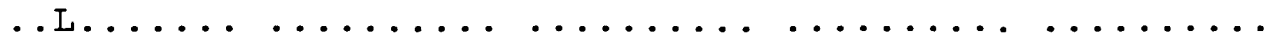

151

200

CAGCACGCCC TGCAGGCCAT CGGTCCGACG CTACTCCTTA ACAGTGACAT CAGCACGCCC TGCAGGCCAT CGGTCCGACG CTACTCCTTA ACAGTGACAT CAGCACGCCC TGCAGGCCAT CGGTCCGACG CTACTCCTTA ACAGTGACAT ..A..R..P. .A..G..H.. R..S..D..A ..T..P..*. .Q..*..H..

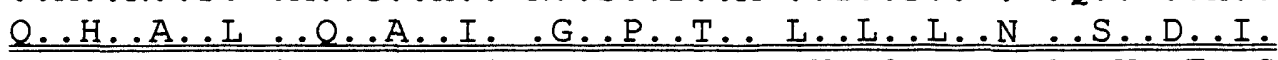
.S.T.P. C.R.P..S .V.R.R. .Y.S.I. . T.V.T.S

201

250

CATCCGGGCA CGCCTGGGGG CCTCCGCACT GGATAGCATC CAAGAGTTCC CATCCGGGCA CGCCTGGGGG CCTCCGCACT GGATAGCATC CAAGAGTTCC $\ldots \ldots \ldots \ldots \ldots \ldots \ldots \ldots \ldots \ldots$.NATAGCATC CAAGAGTTCC $\ldots \ldots \ldots \ldots \ldots \ldots \ldots \ldots \ldots \ldots \ldots \ldots \ldots \ldots \ldots \ldots \ldots$ CA. $\ldots$.

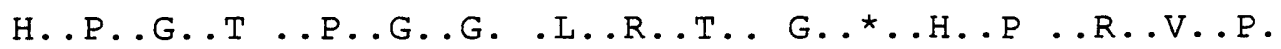

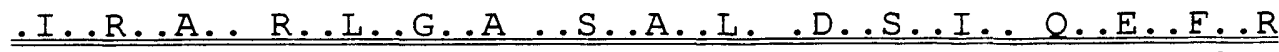
..S.G.H. .A.W..G. P..P.H.W ..I.A.S. .K..S.S.

251

300

GgCTGTCAgG GTGGCTGGCC CAgCAgGAgg ATGCACACCG TATCGTACTC GGCTGTCAGG GTGGCTGGCC CAGCAGGAGG ATGCACACCG TATCGTACTC GGCTGTCAGG GTGGCTGGCC CAGCAGGAGg ATGCACACCG TATCGTACTC GGCTGTCAGG GTNGCTGGCC CAGCAGGAGG ATGCACACCG TATCGTACTC

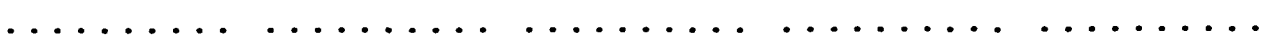
GGCTGTCAGG GTGGCTGGCC CAGCAGGAGG ATGCACACCG TATCGTACTC

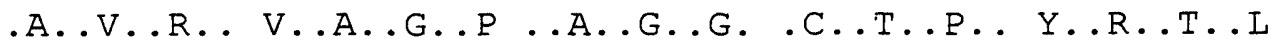

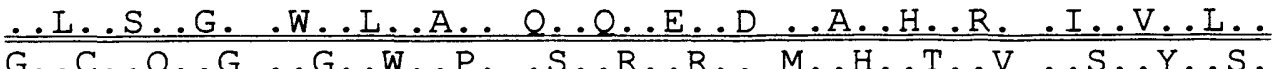


301

$8 \mathrm{~b} 6 \mathrm{a} 16$ t09456 r87932 r89699

h05032

cons

frame I

frame2

frame 3

$8 \mathrm{~b} 6 \mathrm{a} 16$ t09456

r87932

r89699

h05032

cons

frame 1

frame2

frame 3

$8 \mathrm{~b} 6 \mathrm{a} 16$ t09456

r87932

h05032

cons

frame 1

frame 2

frame 3

$8 \mathrm{~b} 6 \mathrm{a} 16$ t09456

r87932

h05032

cons

frame I

frame2

frame 3

t09456

$r 87932$

r89699

cons

frame I

frame 2

frame 3

TACCAGACGG ACGCCTCGCT GACGCCCTGG ACCGTGCGCT GCCTGCGACA TACCAGACGG ACGCCTCGCT GACGCCCTGG ACCGTGCGCT GCCTGCNACA TACCAGACGG ACGCCTCGCT GACGCCCTGG ACCGTGCGCT GCCTGCGACA TACCAGACGG ACGCCTCGCT GACGCCCTGG ACCGTGCGCT GCCTGCGACA $\ldots \ldots \ldots \ldots \ldots \ldots \ldots \ldots \ldots \ldots \ldots \ldots \ldots \ldots \ldots \ldots \ldots \ldots \ldots \ldots$ TACCAGACGG ACGCCTCGCT GACGCCCTGG ACCGTGCGCT GCCTGCGACA ..P..D..G. .R..L..A.. D..A..L..D ..R..A..L. .P..A.T.. Y..Q..T.D...A..S..L..T..P..W. T..V.R..C..L.R..Q.

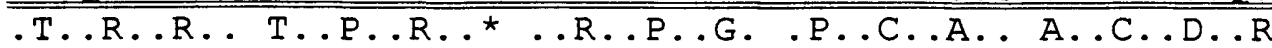

\section{1}

400

G. CCGACTGC ATCCTCATTG TGGGCCTGGG GGACCAGGAG CCTACCCTCG GGCCGACTGC ATCCTCATTG TGGGCCTGGG GGACCAGGAG CCTACCCTCG GGCCGACTGC ATCCTCATTG TGGGCCTGGG GGACAGGAGG CCTACCCTCG GGCCGACTGC ATCCTCATTG TGGGCCTGGG GGACCAGGAG CCT......

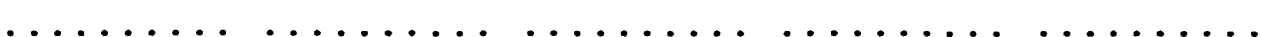
GGCCGACTGC ATCCTCATTG TGGGCCTGGg GGACCAGGAG CCTACCCTCG

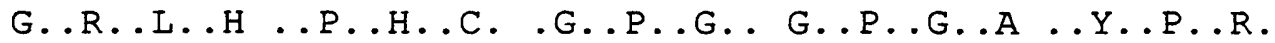
.A..D..C...I..L..I..V ..G..L..G..D..Q.E...P..T..I..G .P.T..A. .S..S.I..W..A.W..G.T.R..S. .I..P.S.

401

GCCANCTGGA GCAGATGCTG GAGAACACGg CTGTGCGCGC CCTTAAGCAG GCCAGCTGGA GCAGATGCTG GAGAACACGG CTNTTCGCGC CCTTAAGCAG GCCAGCTGGA GCAGATGCTG GAGAACACGG CTGTGCGCGC CCTTAAGCAG

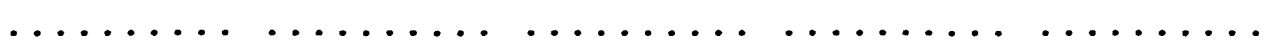
GCCAGCTGGA GCAGATGCTG GAGAACACGG CTGTGCGCGC CCTTAAGCAG .P..A..G.. A..D..A..G ..E..H..G. .C..A.R.. P..*.A..A

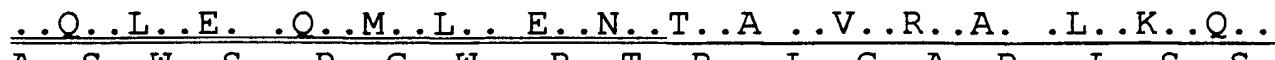
A..S.W.S .R..C.W. .R.T.R.. L..C.A.P .L..S.S.

451 500

CT.GTCCTGC TCCACCGAGA GGANGGCGCG GGCCCC.......... CTAGTCCTTC TTCACCGAGA GGAAGGGCGC GGG............. CTAGTCCTGC TCCACCGAGA GGAGGGCGCG GGCCCACGNA CTCGTTGGGA

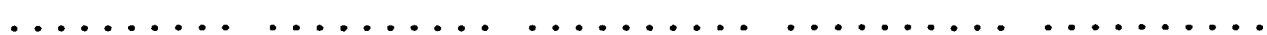
CTAGTCCTGC TCCACCGAGA GGANGGCGCG GGCCCNCGNA CTCGTTGGGA ..S..P..A. .P..P.R.. G..X..R..G ..P.X..X. .S..L..G.

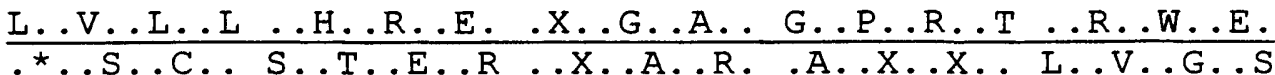
501

$\ldots \ldots \ldots \ldots \ldots \ldots \ldots \ldots \ldots \ldots \ldots \ldots \ldots \ldots \ldots \ldots \ldots$ GTGGCTAAAT ATGCGCATGG GTGCTCGGGG CACTGCACCT .......

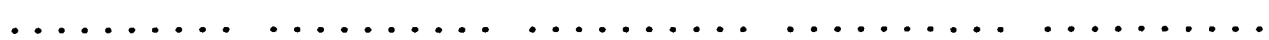
GTGGCTAAAT ATGCGCATGG GTGCTCGGGG CACTGCACCT NNNNNNNNNN V..A..K..Y ..A..H..G. .C..S..G.. H..C..T.X ..X..X..X.

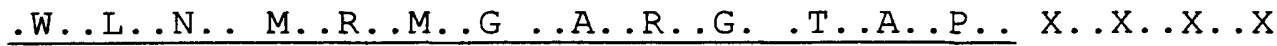
.G..*.I. .C..A.W. V..L.G.A..I.H.I. .X..X..X.. 
r89699

h05032

r56734

r13907

t23706

cons

frame 1

frame2

frame 3

r89699

h05032

r56734

r13907

t23706

f12067

r25505

h09837

h10469

r17727

cons

frame 1

frame2

frame 3

r89699

h05032

r56734

r13907

t23706

f12067

r25505

h0983.7

h10469

r17727

t66368

cons

frame 1

frame2

frame 3

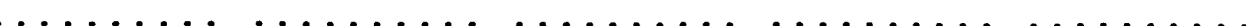

$\ldots \ldots \ldots \ldots \ldots \ldots \ldots \ldots \ldots \ldots \ldots \ldots \ldots$ ACTGCACCTG

$\ldots \ldots \ldots \ldots \ldots \ldots \ldots \ldots \ldots$. $\ldots \ldots$. . . . . .

$\ldots \ldots \ldots \ldots \ldots \ldots \ldots \ldots \ldots \ldots \ldots \ldots$.GCTCGGGGC ACTGCACCTG

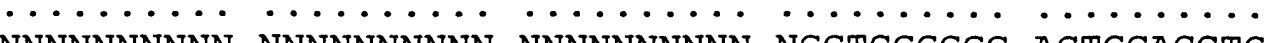
NNNNNNNNNN NNNNNNNNNN NGCTCGGGGC ACTGCACCTG

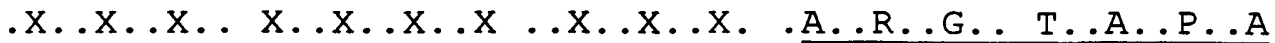
..X.X..X. .X..X..X..X..X.X..X ..L.G.A. .L.H.I. $X \ldots X \ldots X \ldots X \ldots X \ldots X \ldots X . . X \ldots X \ldots X \ldots X \ldots S \ldots G \ldots H \ldots C \ldots T \ldots C$.

601

650

$\ldots \ldots \ldots \ldots \ldots \ldots \ldots \ldots \ldots \ldots \ldots$ CGCTGTCCGC GCGCCTNTTT TCGCGCCGCA GCCCTGCCAA GCTGCATGAG CGCTGTCCGC GCGCCTCTTT TCGCGCCGCA GCCCTGNCAA GCTGCATGAG CGCTGTCCGC GCGCCTCTTT TCGCGCCGCA GCCCTGCCAA GCTGCATGAG

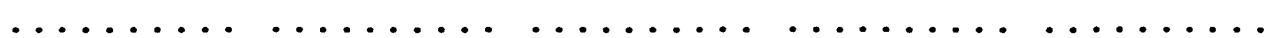

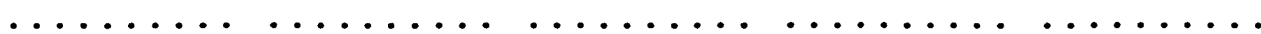
$\ldots \ldots \ldots \ldots \ldots \ldots \ldots$. CGCGCCGC AGCCCTGCCA AGCTGCATGA $\ldots \ldots \ldots \ldots \ldots \ldots \ldots \ldots \ldots \ldots \ldots \ldots \ldots \ldots \ldots \ldots$ $\ldots \ldots \ldots \ldots \ldots \ldots \ldots \ldots \ldots \ldots \ldots$ NNNNN NNNNNNNNNN

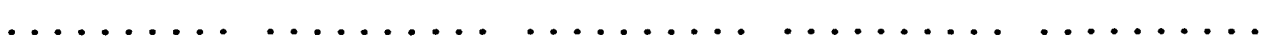
CGCTGTCCGC GCGCCTCTTT TCGCGCCGCA GCCCTGNCAA GCTGCATGAG

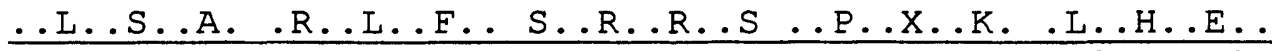

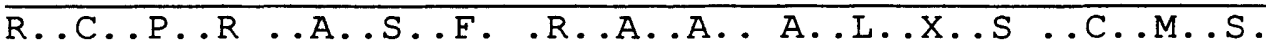

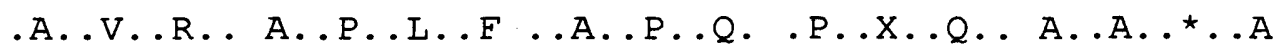

651

700

$\ldots \ldots \ldots \ldots \ldots \ldots \ldots \ldots$ CTCTACGAGA AGGTTTTNC CAGGCGNGCG GACCGGCACA GCGACTTCTC CTCTACGAGA AGGTTTTCTC CAGGCGCGCG GACCGGCACA GCGACTTCTC ........AA GCTTGGCACG AGGGGCGCGC GGACCGGCAC AGCGACTTCT

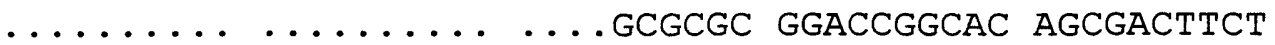
GCTCTACGAG AAGGTTTTCT CCAGGCGCGC GGACCGGCAC AGCGACTTCT GCTCTACGAG AAGGTTTTCT CCAGGCGCGC GGACCGGCAC AGCGACTTCT NNNNNNNNNN NNNNNNNNNN NNNNNNNNNN NNNNNNNNNN NNNNNNNNNN $\ldots \ldots \ldots \ldots \ldots \ldots \ldots$............

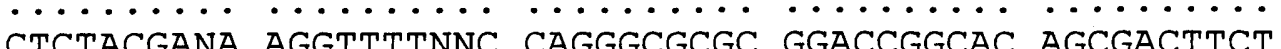

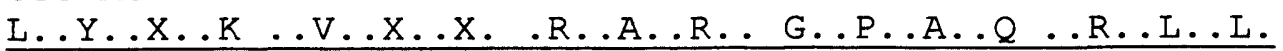

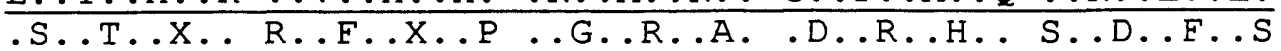
..L.R..X. .G.F..X.. Q..G..A.R .T.G.T. .A.T..S. 
r89699

h05032

r56734

r13907

t23706

f12067

r25505

h09837

h10469

r17727

t66368

t10299

cons

frame 1

frame2

frame 3

h05032

r56734

r13907

t23706

f 12067

r25505

h09837

h10469

r17727

t66368

t10299

cons

frame 1

frame2

frame 3

h05032

r56734

r13907

t23706

f12067

r25505

h09837

h10469

r17727

t66368

t10299

h58564

cons

frame 1

frame2

frame 3 NTE-pep

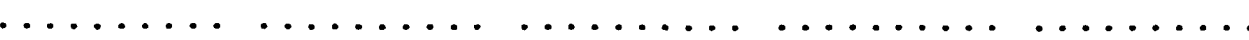

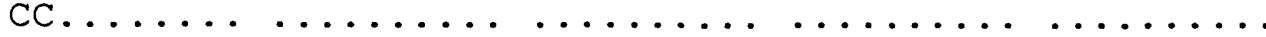

CCGCTTGGCA GGGTGCTCAC GGGGAACACC ATTGCCCTTG TGCTAGGNGG CCGCTTGGCA GGGTGCTCAC GGGGAACACC ATTGCCCTTG TGCTAGGCGG CCCGCTTGGC AGGGTGCTCA CGGGGAACAC CATTGCCCTT GTGCTAGGCG CCCGCTTGGC AGGGTGCTCA CGGGGAACAC CATTGCCCTT GTGCTAGGCG CCCGCTTGGC AGGGTGCTCA CGGGGAACAC CATTGCCCTT GTGCTAGGCG CCCGCTTGGC AGGGTGCTCA CGGGGAACAC CATTGCCCTT GTGCTTGGCG CCCGCTTGCG AGGGTGCTCA CGGGGAACAC CATTGCCCTT GTGCTAGGGG CCCGCTTGGC AGGGTGCTCA CGGGGAACAC CATTGCCCTT GTGCTAGGNT .......... TGCTCA CGGGGAACAC CATTGCCCTT GTGCTAGGNG

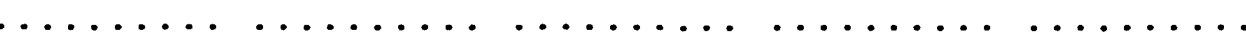
CCCGCTTGGC AgGgTGCTCA CGgGGAACAC CATTGCCCTT GTGCTAGGCG .P..L..G..R..V..L.T ..G..N..T..I..A..L..V..L.G..G

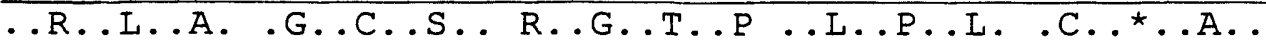
P.A..W..Q ..G.A..H. .G.E.H. H..C.P.C .A.R.R.

751

800

GGGCGGGGNC AGGGNTGCCT CGCACATCGG AGTACTAAAG GCATTAGANG GGGC

GGGGCGGGGC CAGGGGCTGC TCGCACATCG GAGTACTAAA GGCATTAGAG GGGGCAGGGG CTGC...... TCGCACATCG GAGTACTAAA GGCATTAGAG GGGGCGGGGC CAGGGGCTGC TCGCACATCG GAGTACTAAA GGCATTAGAG AGGGCGGGGC CAGGG..TGC TCGCACATCG GAGTACTAAA GGCATTAGAG GGGGCCGGGC CAGGGGCTGC TCGCACATCG GAGTACTAAA GGCATTAGAG GGGGCGGGG. CAGGGC.TC. TCGCACATCG GAGTACTAAA GGCATTAGAG GGGNC.GGGG CAGGGCGTGC TCGCACATCG GAGTACTAAA GGCATTAGAG GGGGCGGGGC CAGGGGCTGC TCGCACATCG GAGTACTAAA GGCATTAGAG

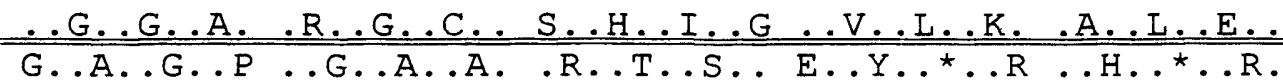

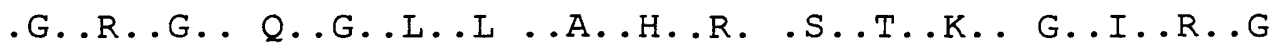

801

850

AGGCGGGGGT NCCC. . TNGA CTNGTGGGCG GCACGTNCAT TGGCTCTNTC GAGGCGGGGG TCCCCGTGGA CCTGGTGGGC GGTACGTCCA TTGGCTCTTT GAGGCGGGGG TCCCCGTGGA CCTGGTGGGC GGCACGTCCA TTGGCTCTTT GAGGCGGNGG TCCCCGTGGA CCTGGTGG.C GGCACGTCCA TTAGCTCTTT GAGGCGGGGG TCCCCGTGGA CCTGGTGGGC GGCACGTCCA TTGGCTCTTT GAGGCGGGGG TCCCC.TGGA CCTGGTGGGC GGCACGTCCA TTGGCTCTTT GAGGCGGGGN TCCCCGTGGA CCTGGTGGGC GGCACGTCCA TTGGCTCTTT GAGGCGGGGT TCCCCGTGGA CCTGGTGGGC GGCACGTCCA TTGGCTCTTT

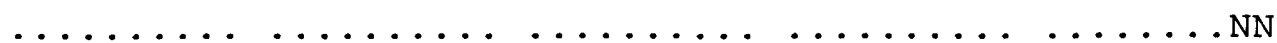
GAGGCGGGGG TCCCCGTGGA CCTGGTGGGC GGCACGTCCA TTGGCTCTTT

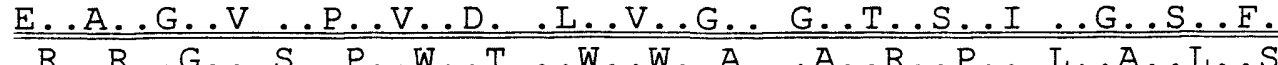
..G..G.G. .P..R..G. P..G..G..R ..H..V..H. .W..L.F. $\ldots A \ldots G \ldots V \ldots P \ldots V \ldots A . . I \ldots V \ldots G \ldots$. . . . . . . . . . 
h05032

r56734

r13907

t23706

f12067

r25505

h09837

h10469

r17727

t66368

t10299

h58564

cons

frame 1

frame2

frame 3

f12067

r25505

h09837

h10469

r17727

t66368

t10299

h58564

cons

frame 1

frame2

frame 3

f 12067

r25505

h09837

h10469

r17727

t66368

t10299

h58564

cons

frame 1

frame2

frame 3

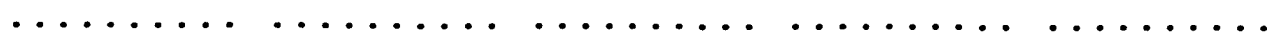

ATCGGAGCGT TGTACGGTAG G................

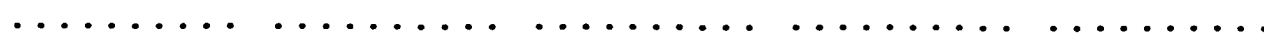

CATCGGAGCG TTGTACGCGA GGAG

CATCGGAGCG TTGTACGCGG AGAGCGCACC CGAGCCGCAC GAAGCAGCGG

CATCGGAGCG TTGTACGCGA GGAGCGCACT CAAGCCG.AC GAAGCAGCGG

CATCGGAGCG TTGTACGCGA GGAGCGCAGC CCA.CCGCAC GAAGCAGCGG

CATCGGAGCG TTGTACGCGA GGAGCGCAGC CCAGCCGCAC GAAGCAGCGG

CATCGGAGCG TTGTACGGGA GGAGCGCA.C CAG.CCGCAC GAAGCAGCGG

CATCGGAGCG TTGTACGGNA GGAGCGCAGG CAAGCCGCAC GAAGCAGCGG NNNNNNNNNN NNNNNNNGGA GGAGCGCAGC GCAGCCGCAC GAAGCAGCGG $\ldots \ldots \ldots \ldots \ldots \ldots \ldots \ldots$ CATCGGAGCG TTGTACGCGA GGAGCGCAGC CNAGCCGCAC GAAGCAGCGG

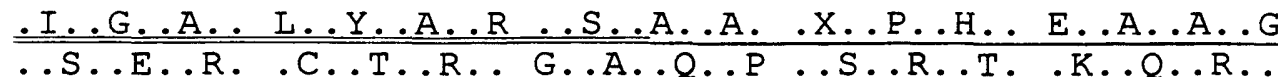

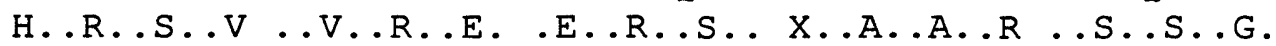

901

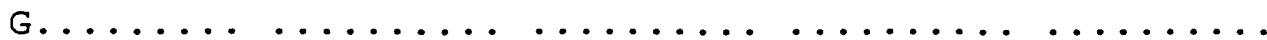

GCCCCGGAGT TGGCAAGAGC ATGACTTCGg TTCTGGNACC TGTNTTGGAA GCCCGGGAGT GGGCAAGAGC ATGACTTCGG TGCTGGAACC TGTGTTGGAC GCCCGGGAGT TGGCAAGAGC ATGACTTCGG TGCTNGAACC TGTGTTNGAC GCCCGGGAGT GGGCAAGAGC ATGACTTCGG TGCTGGAACC TGTGTTGGAC GCCCGGGAGT GGGCAAGAGC ATGACTTCGG TGCTGGAACC TGTGTTGGAC GCCCGGGAGT GGGCAAGAGC ATGACTTCGG TGCTGGAACC TGTGTTGGAC

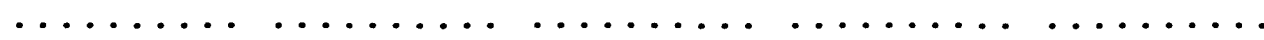
GCCCGGGAGT GGGCAAGAGC ATGACTTCGG TGCTGGAACC TGTGTTGGAC ..P..G..V. .G..K..S. . M..T..S..V ..L..E..P. .V..L..D..

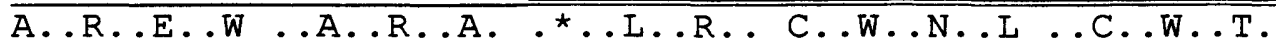
.P..G..S..G..Q.E..H ..D.F.G. .A.G.T.. C..V.G..P

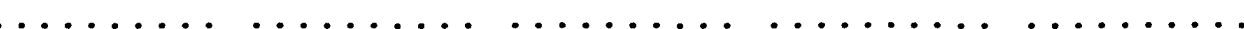

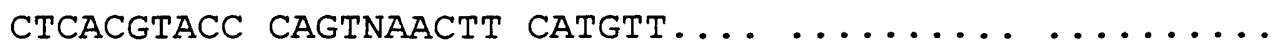
CTCACGTACC CAGTCACCTC CATGTTCACT GGGTCTGCCT TTAACCGNAG CTCACGTACC CAGTCACCTN CATGTTCACT GGGTCTGCCT TTAACCGCAG CTCACGTACC CAGTCACCTC CATGTTCACT GGGTCTGCCT TTAACCGCAG CTCACGTACC CAGTCACCTC CATGTTCACT GGGTCTTCCT TTAACCGCAG CTCACGTACC CAGTCACCTC CATGTTCACT GGGTCTGCCT TTAACCGCAG

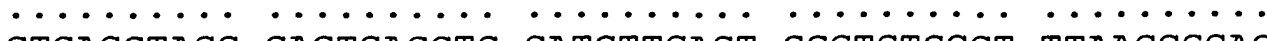
CTCACGTACC CAGTCACCTC CATGTTCACT GGGTCTGCCT TTAACCGCAG

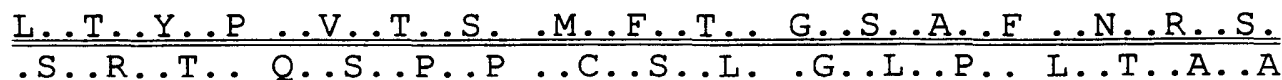
..H.V..P. .S.H..L.. H..V..H..W ..V..C.L. .*.P.Q.. 
r25505

h09837

h10469

r17727

t66368

t10299

h58564

r51195

r15042

cons

frame 1

frame2

frame 3

NTE-pep

h09837

h10469

$r 17727$

t66368

t10299

h58564

r51195

r15042

h61615

r51470

cons

frame 1

frame2

frame 3

NTE-pep

t66368

t10299

h58564

r51195

r15042

h61615

r51470

cons

frame 1

frame2

frame 3

$t 66368$

t10299

h58564

r51195

$r 15042$

h 61615

r51470

cons

frame 1

frame 2

Erame 3

NTE-pep

CATCCATCGG GT.

CATCCATCGG GTCTTTCAGG NTAAGCAGAT TNAGGAC.TG TTGCTGC.TT

CATCCATCGG GTCTTCCAGG ATAAGCAGAT TGAGGACCTG TGGCTGCCTT

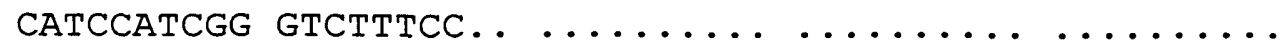

CATCCATCGg GTCTTCCAGg ATAAGCAGAT TGAGGACCTG TGGCTGCCTT ................ AGG ATAAGCAGAT TGAGGACCTG TGGCTGCCTT $\ldots \ldots \ldots \ldots \ldots \ldots \ldots \ldots$. $\ldots \ldots \ldots$ TGAGACCTG TGGCTGCCTT

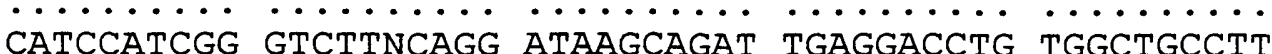

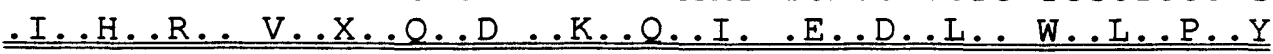
I.S.I..G. .S.X..R.. I..S.R..L ..R.T..C. .G..C.L.

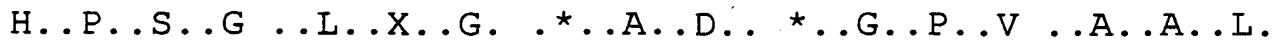
$\ldots \ldots \ldots \ldots \ldots \ldots \ldots \ldots \ldots \ldots \ldots \ldots \ldots \ldots$..........

1051

1100

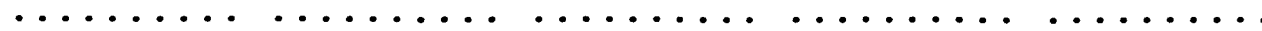

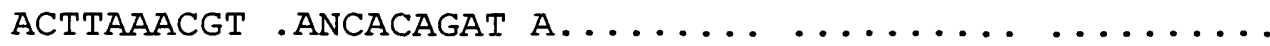

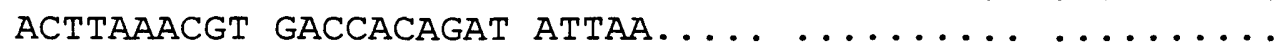

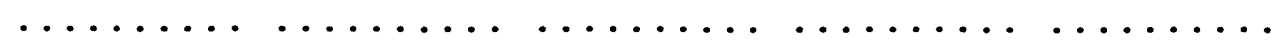

ACTTCAACGT GACCACAGAT ATCACCGCCT CAGCCATGCG AGTCCACAAA ACTTCAACGT GACCACAGAT ATCACCGCCT CAGCCATGCG AGTCCACAAA ACTTCAACGT GACCACAGAT ATCACCGCCT CAGCCATGCG AGTCCACAAA $\ldots \ldots \ldots \ldots \ldots \ldots \ldots \ldots \ldots \ldots \ldots \ldots \ldots \ldots \ldots \ldots \ldots$ $\ldots \ldots \ldots \ldots \ldots \ldots \ldots \ldots \ldots \ldots \ldots \ldots \ldots \ldots \ldots$ AGTCCACAAA

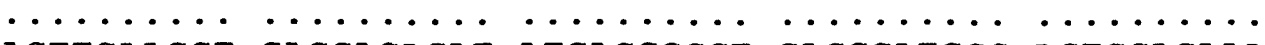
ACTTCAACGT GACCACAGAT ATCACCGCCT CAGCCATGCG AGTCCACAAA

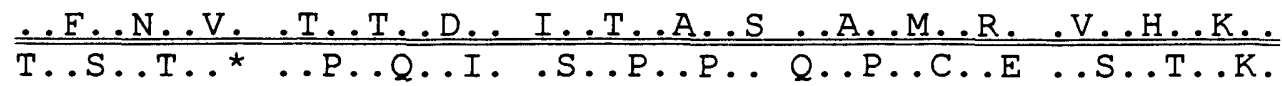

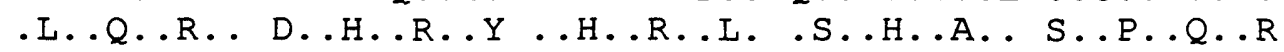

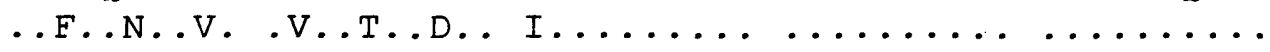

1101

1150

GATGGCTCCC TGTGGCGGTA CGTGCGCGCC AGCATGACGC TGTCGGGCTA GATGGCTCCC TGTGGCGGTA CGTGCGCGCC ANATG.ACGC TGTCGGGCTA GATGGCTCCC TGTGGCGGTA CGTGCGCGCC ANATGGACGC TGTCGGGCTA GATGGCTCCC TGTGGCGGTA CGTGCGCGCC ANATNGACGC TGTCGGGCTA GATGGCTCCC TGTGGCGGTA CGTGCGCGCC ACATGGACGC TGTCGGGCTA $\ldots \ldots \ldots \ldots \ldots \ldots \ldots$ GATGGCTCCC TGTGGCGGTA CGTGCGCGCC ANATGGACGC TGTCGGGCTA

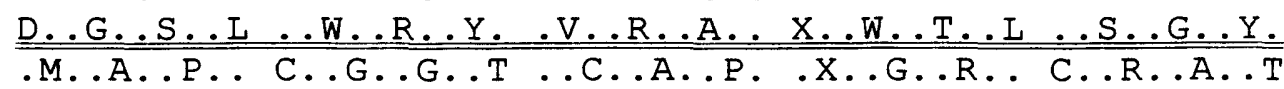
.W..L..P. .V..A..V.. R..A..R.X ..M.D..A. .V..G..L.

1151

1200

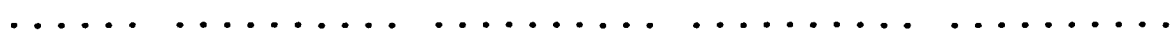

CCTGCCCC.G CTGTGCGACC CAAGGACGGG CACCTACTCA TGGATG.... CCTGCCCCCG CTGTG. ACCC CAAGGACGGG CACCTACTCA TGGATGGCGG CCTGCCCCCG CTGTGNACCC CAAGGACGGG CACCTACTCA TGGATGGCGG CCTGCCCCCG CTGTGNACCC CAAGGACGGG CACCTACTCA TGGATGGCGG CCTGCCCCCG CTGTGACCCC CAAGGACGGG CACCTACTCA TGGATGGCGG

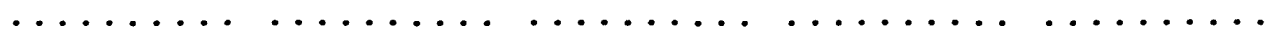
CCTGCCCCCG CTGTGNACCC CAAGGACGGG CACCTACTCA TGGATGGCGG .L..P..P.. L..X..T..P ..R..T..G. .T.Y..S. . W..M..A..A

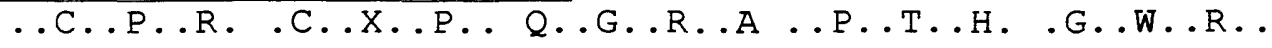
P.A..P.A $\ldots V \ldots X \ldots P . \quad$ K..D.G..H.L.L..M.D.G..G. $\ldots \ldots \ldots \ldots \ldots \ldots \ldots$...........L.T.W..G.G. 
$t 10299$

h58564

r51195

r15042

h 61615

r51470

cons

frame 1

frame2

frame 3

NTE-pep

$t 10299$

h58564

r51195

r 15042

h61615

r51470

r 45802

cons

frame 1

frame2

frame 3

$t 10299$

h58564

r51195

r15042

h 61615

r51470

r45802

cons

frame 1

frame2

frame 3

r51195

r15042

h61615

r51470

r45802

cons

framel

frame2

frame 3 CTACATCAAC AATCTGCCA CGGACATCGC CCGCAGCATG GGTGCCAAAA CTACATCAAC AATCTGCCAG CGGACATCGC CCGCAGCATG GGTGCCAAAA CTACATCAAC AATCTGCCAG CGGACATCGC CCGCAGCATG GGTGCCAAAA CTACATCAAC AATCTGCCAG CGGACATCGC CCGCAGCATG GGTGCCAAAA

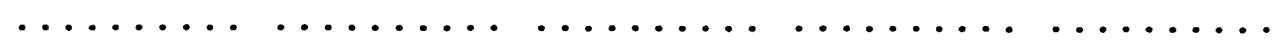
CTACATCAAC AATCTGCCAG CGGACATCGC CCGCAGCATG GGTGCCAAAA ..T..S.T. .I..C.Q.. R..T..S..P ..A..A..W. .V..P.K.. L..H..Q.Q ..S..A..S. .G..H..R.. P.Q..H.G ..C.Q..N.

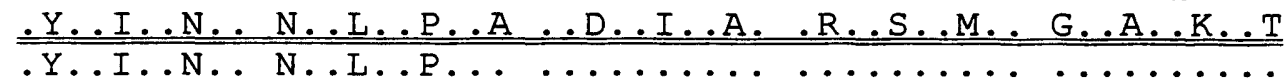

\section{1}

1300

$\ldots \ldots \ldots \ldots \ldots \ldots \ldots \ldots \ldots \ldots \ldots$ CGGTCATCGC CATTGACGTG GGGAGCCAGG ATGAGACGGA CCTCAGCACC CGGTCATCGC CATTGACGTG GGGAGCCAGG ATGAGACGGA CCTCAGCACC CGGTCATCGC CATTGACGTG GGGAGCCAGG ATGAGACGGA CCTCAGCACC CGGTCATCGC CATTGACGTG GGGAGCCAGG ATGAGACGGA CCTCAGCACC $\ldots \ldots \ldots \ldots \ldots \ldots \ldots \ldots \ldots \ldots \ldots \ldots \ldots$. TGAGACGGA CCTCAGCACC

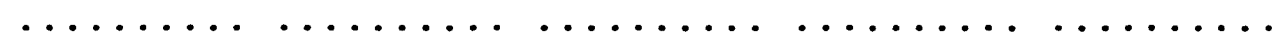
CGGTCATCGC CATTGACGTG GGGAGCCAGG ATGAGACGGA CCTCAGCACC R..S..S..P ..L..T..W. .G..A..R.. M..R..R.T ..S.A..P. .G..H..R..H..* .R..G ..E..P..G. .* .D..G.. P..Q..H..L

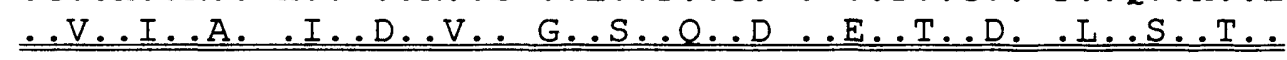

1301

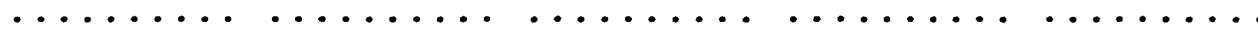

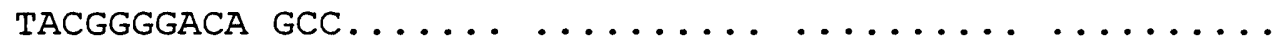

TACGGGGACA GCCTGTTCGG CTGGTGGCTG CTGTGGAAGC GG....... TACGGGGACA GCCTGTCCGG CTGGTGGCTG CTGTGGAAGC GGCTGAATCC TACGGGGACA GCCTGTCCGG CTGGTGGCTG CTGTGGAAGC GGCTGAATCC TACGGGGACA GCCTGTCCGG CTGGTGGCTG CTGTGGAAGC GGCTGAATCC

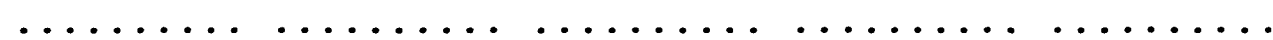
TACGGGGACA GCCTGTCCGG CTGGTGGCTG CTGTGGAAGC GGCTGAATCC .T..G..T..A..C..P..A ..G..G..C. .C..G..S.. G..*....P ..R..G..Q. .P..V.R.. L..V..A..A .V..E..A. .A.E..S. Y..G.D..S ..L..S..G. .W..W..L.. L..W..K..R ..L..N..P.

\section{1}

1400

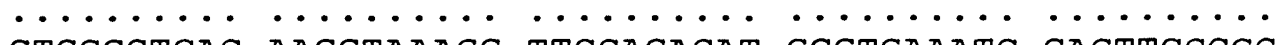
CTGGGCTGAC AAGGTAAAGG TTCCAGACAT GGGTGAAATC CAGTTCCCGC CTGGGCTGAC AAGGTAAAGG TTCCAGACAT GGCTGAAATC CAGTNCCGCC CTGGGCTGAC AAGGTAAAGG NTNCAGACAT AGCTGAAATT CAGTTCCGC.

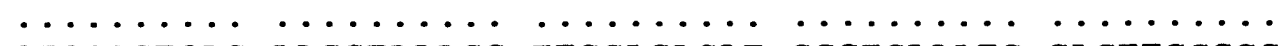
CTGGGCTGAC AAGGTAAAGG TTCCAGACAT GGCTGAAATC CAGTTCCGCC

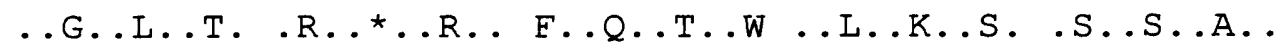

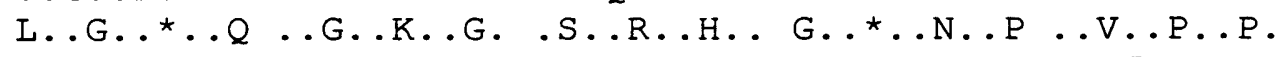
.W..A..D..K..V..K..V ..P..D..M..A..E..I.. Q..F..R.L 
r51195

r 15042

h61615

r51470

$r 45802$

$r 54769$

$r 48493$

$r 47780$

m78841

cons

frame 1

frame2

frame 3

NTE-pep

r15042

h61615

r51470

$r 45802$

r54769

r48493

r47780

m78841

cons

frame 1

frame2

frame 3

NTE-pep

r 45802

r54769

$r 48493$

$\Upsilon 47780$

m78841

3 prm

cons

frame 1

frame2

frame 3

NTE-pep

$r 51470$

$r 45802$

r54769

r48493

$r 47780$

m78841

3 prm

cons

framel

frame2

frame 3

TCPCACGT

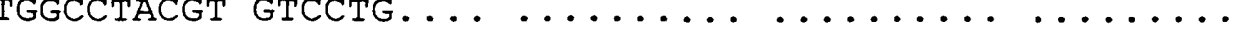
TGGCCTACGT GTCCTGTGTT GCGGCATNAG AAGGTTGTCA AGTCCAGTTC

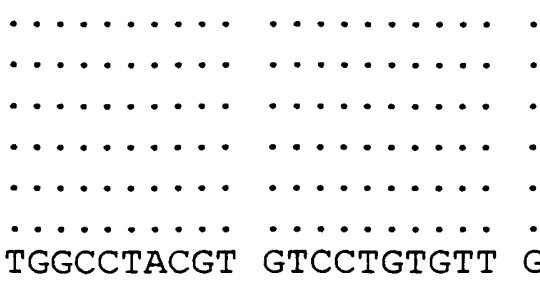

$\ldots \ldots \ldots \ldots \ldots$ . GGCAGCTA GAGGTTGTCA AGTCCAGCTC - GGGCAGCTA GAGGTTGTCA AGTCCAGCTC . NGGCAGCTA GAGGTTGTCA AGTCCAGCTC TGGCCTACGT GTCCTGTGTT GNGGCAGCTA GAGGTTGTCA AGTCCAGCTC W..P.T..C ..P..V..L. .X. Q..L..E..V.V..K ..S.S..S.

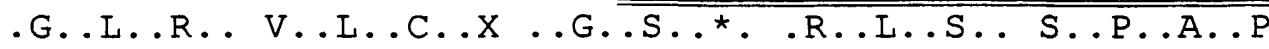
.A..Y..V..S.C..V.. X..A..A..R ..G..C.Q. .V.Q..L..

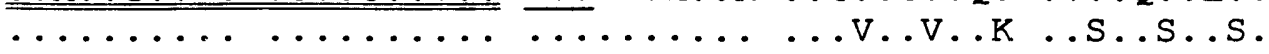

1451

1500

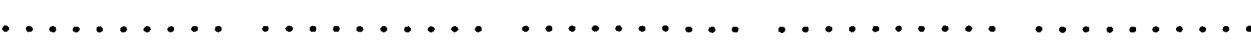

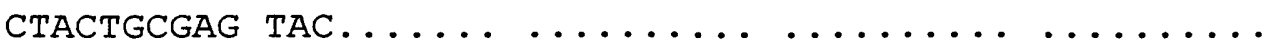

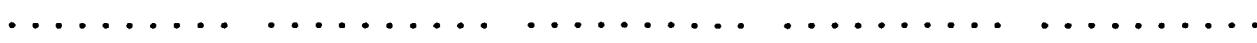

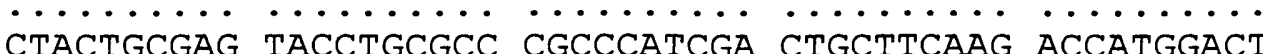
CTACTGCGAG TACCTGCGNC CGCCCATCGA CTGCTTCAAG ACCATGGACT CTACTGCGAG TACCTGCGCC CGCCCATCGA CTGCTTCAAG ACCATGGACT

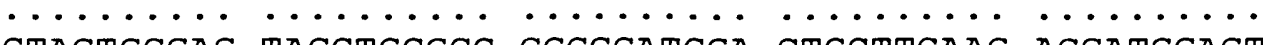
CTACTGCGAG TACCTGCGCC CGCCCATCGA CTGCTTCAAG ACCATGGACT

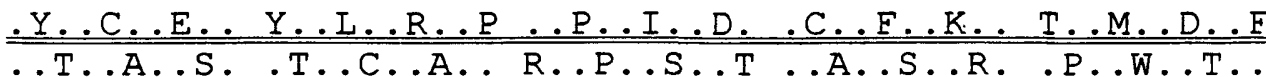

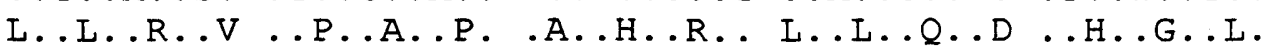

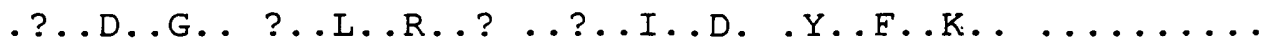

1501

1550

TTGGGAAGTT CGACCAGATC TATGATGTGG GCTACCAGTA CGGGAAGGCG TTGGGAAGTT CGACCAGATC TATGATGTGG GCTACCAGTA CGGGAAGGCG TTGGGAAGTT CGACCAGATC TATGATGTGG GCTACCAGTA CGGGAAGGCG $\ldots \ldots \ldots \ldots \ldots \ldots \ldots \ldots \ldots \ldots \ldots \ldots \ldots$ TA CGGGAAGGCG $\ldots \ldots \ldots \ldots \ldots \ldots \ldots$ .G..K..F. .D..Q..I.. Y..D..V..G ..Y..Q..Y. .G..K..A.. L..G.S..S .T.R..S. .M..M.W.. A.T..S.T ..G.R.R.

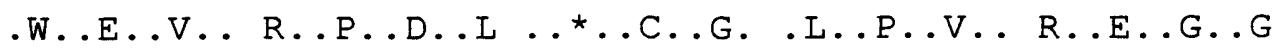
........ .D..Q.I.. Y..D..V.G .Y..Q.Y. .G..K....

1551

1600

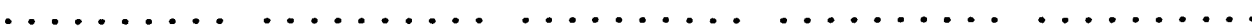

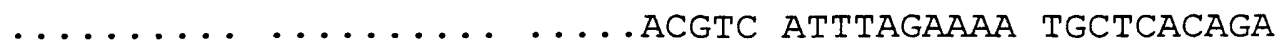
GTGTTTGGAG GCTGGAGCCT TGGCAACGTC ATTGAGAAAA TGCTCACAGA GTGTTTGGAG GCTGGAGCCG TGGCAACGTC ATTGAGAAAA TGCTCACAGA GTGTTTGGAG GCTGGAGCNT NGGCAACGTC ATTGAGAAAA TGCTCACAGA GTGTTTGGAG GCTGGAGCCG TGGCAACGTC ATTGAGAAAA TGCTCACAGA

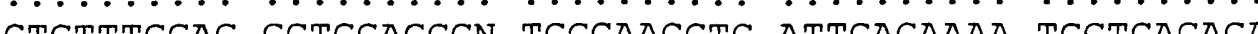
V..F..G.G ..W..S..X. .G..N..V.. I..E..K..M ..L.T..D. .C.I.E.. A..G.A..X .A.T.S. .L.R.K.. C.S.Q.T .V..W..R. .L.E..P. W. W..R.H ..*..E.N. .A..H.R. 
$r 51470$ $r 45802$ $r 54769$ r48493 $r 47780$ m78841

3 prm

cons

frame 1

frame2

frame 3

$r 51470$

$r 45802$

r54769

r48493

$r 47780$

m78841

3prm

cons

frame 1

frame2

frame 3

$r 51470$

$r 45802$

r54769

r48493

r 47780

m78841

3prm

cons

frame 1

frame2

frame 3

r45802

r54769

r48493

r 47780

m78841

3 prm

cons

frame1

frame 2

frame 3

$r 47780$

m78841

3 prm

cons

frame 1

frame 2

frame 3

CCGGCGG.CT ACAGACCTTA ATGAGAGCCG CCGTGCAGAC GTGCTTGCCT CCGGCGGTCT ACAGACCTTA ATGAGAGCCG CCGTGCAGAC GTGCTTGCCT CCGGCGGTCT ACAGACCTTA ATGAGAGCCG CCGTGCAGAC GTGCTTGCCT CCGGCGGTTT ACAGACCTTA ATGAGAGCCG CCGTGCAGAC GTGCTTGCCT CCGGCGGTCT ACAGACCTTA ATGAGAGCCG CCGTGCAGAC GTGCTTGCCT

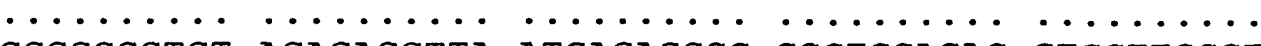

1651

1700

$\ldots \ldots \ldots \ldots \ldots \ldots \ldots \ldots \ldots \ldots \ldots$

TCCTAAGCTC TGGCTTCACT GACTTGGCAT AGATTGTGTC CCGGATTGAG TCCCAAGCTC TGGCTTCACT GACTTNGCAG AGATTGTGTC CCGGATTGAG TCCCAAGCTC TGGCTTCACT GACTTGGCAG AGATTGTGTC CCGGATTGAG TCCCAAGCTC TGGCTTCACT GACTTGGCAG AGATTGTGTC CCGGATTGAG TCCCAAGCTC TGGCTTCACT GACTTGGCAG AGATTGTNTC CCGGATTGAG

TCCCAAGCTC TGGCTTCACT GACTTGGCAG AGATTGTGTC CCGGATTGA

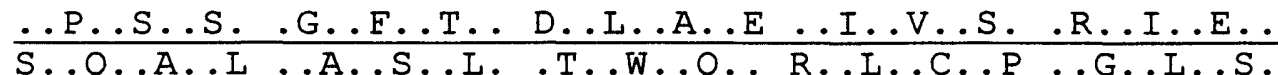
.P..K..L. W..L..H..* ..L..G.R. .D..C..V.. P..D....A

1701

1750 CCCCCCACGA GCTATGTCTC TTTGGCTGT. CTG.ACGGAG AG....... CCCCCCACGA GCTATGTCTC TGTGGCTGTG CTTGACGGAG AGGAGTCAGA CCCCCCACGA GCTATGTCTC TGTGG.TGTG CTGGACGGAG AGGAGTCAGA CCCCCCACGA GCTATGTCTC TTTGGCTGTG CTT.ACGGAG AGG....... CCCCCCACGA GCTATGTCTC TNATGGCTGT GCTGACGGAG AGGAGTCAGA

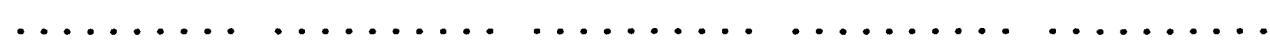
CCCCCCACGA GCTATGTCTC TNTGGCTGTG CTTGACGGAG AGGAGTCAGA P..P.T..S ..Y.V..S. .X..A.V.. L..D.G.E ..E..S.D.

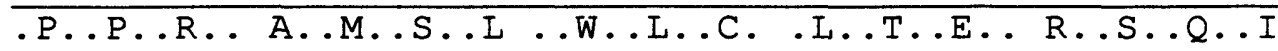
..P..H.E. .I..C.I..X..G..C..A ..*.R..R. .G..V.R.

1751

1800

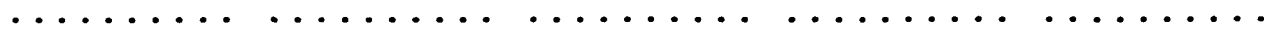

TTGTCTTGAA GAGTATGAGG AGGA................

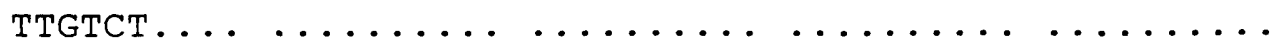

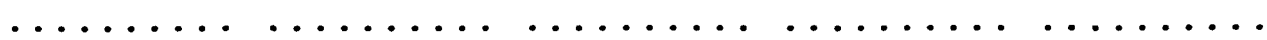

TTGTCTGACA GAGTATGAGG AGGACGCCGG ACCCGACTGC TCGAGGGATG

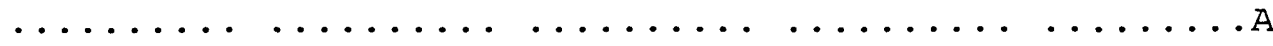
TTGTCTNNNA GAGTATGAGG AGGACGCCGG ACCCGACTGC TCGAGGGATN

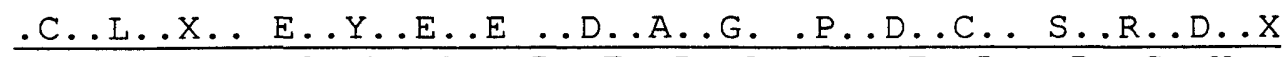
.V..X.X. .S.M..R.. R..T.P.D .P.T.A. .R.G.X..

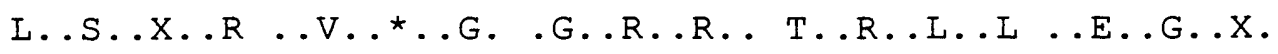

1801

1850

AAGGGGGGTN CCCCGAGGGC GCAA... CCCA GCACTGCCTC CGAGATGGAG AGGGGGGTTC CCCCGAAGGC GCAANCCCCA GCACTGCCTC CGAGATGGAG ANGGGGGNTN CCCCGANGGC GCAANCCCCA GCACTGCCTC CGAGATGGAG

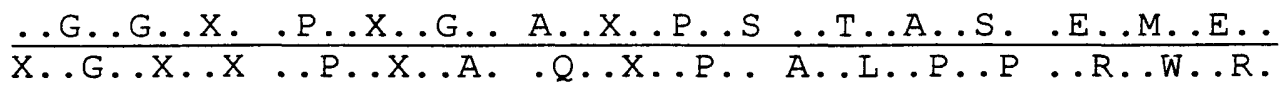

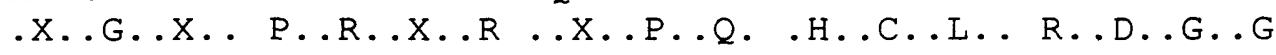


r 47780

$\mathrm{m} 78841$

3 prm

cons

frame 1

frame2

frame 3

$r 47780$

m78841

3 prm

cons

frame 1

frame2

frame 3

$r 47780$

m78841

3 prm

cons

frame 1

frame2

frame 3

m78841

$3 p r m$

cons

frame 1

frame2

frame 3

$\mathrm{m} 78841$

3 prm

cons

frame 1

frame2

frame 3

3prm

cons

frame 1

frame 2

frame 3

GAGGAGAAGT CGATTCTCCG GCAACGACGC TGTCTGCCCC AGGAGCGCCC GAGGAGAAGT CGATTCTCCG GCAACGANGC TGTCTGCCCC AGGAGCGCCC GAGGAGAAGT CGATTCTCCG GCAACGANGC TGTCTGCCCC AGGAGCGCCC E..E..K..S ..I..L..R. .Q..R..X.. C..L..P..Q ..E..R..P.

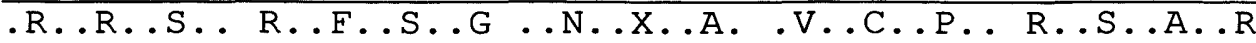
..G..E..V. .D..S..P..A..T..X..L ..S..A..P. .G..A..P..

1901

GGCTCAGCCA CAGATGCCTN AGGACCTCGA CAAGGGTCA. CCCCTCC.TC GGCTCAGCCA CAGATGCCTG AGGACCTCGA ACAGGGTCAC CCCCTCCCTC GGCTCAGCCA CAGATGCCTN AGGACCTCGA NNAGGGTCAC CCCCTCCCTC

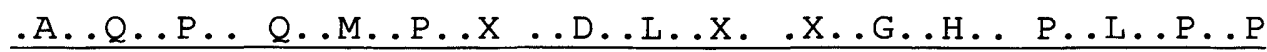
..L..S.H. .R..C.L.. R.T..S.X ..R..V.T. .P..S.L..

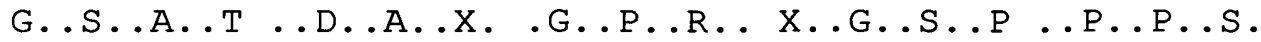

1951 CCACCCCTGG ACTGGGCTGG GGGTGNNCCC GTGGGGGTAG CTCACTCCCC CCACCCCTGG ACTGGNNTGG GGGTGNNCCC GTGGGGGTAG CTCACTCCCC

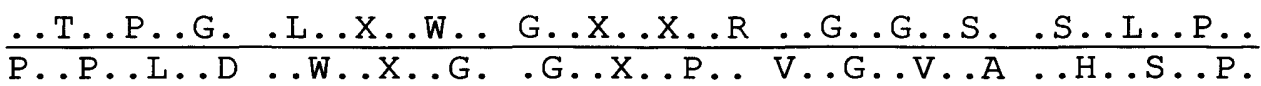

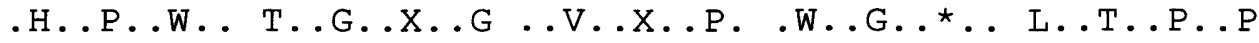

2001

2050

CTCCTGCTGC TATGCCTGTG ACCCCCGCGG CCCACACACT GGACTGACCT CTCCTGCTGC TATGCCTGTG ACCCCCGCGG CCCACACACT GGACTGACCT

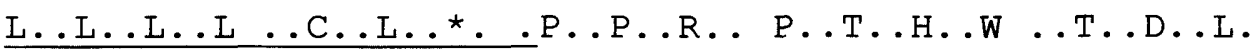

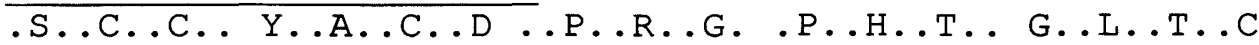
.P.A..A. .M..P..V. T..P.A..A ....T.L. .D.*.P.

2051

GCCCTGAGCG GGGATGCAGT GTTGCACTGA TGACTTGACC AGCCCCTCCC GCCCTGAGCG GGGATGCAGT GTTGCACTGA TGACTTGACC AGCCCCTCCC .P..*..A.. G..M..Q..C ..C....D. .D..L.T.. S..P....P ..P..E..R. .G..C..S..V..A..L..M ..T.*..P. .A..P..P..

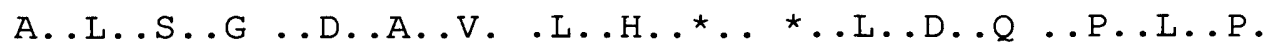

2101

2149

CCAATAAACT CGCCTCTTGG AAAAAAAAAA ............

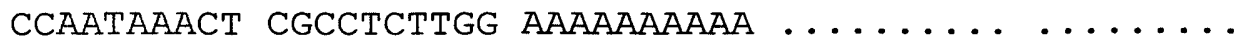

$\ldots$...K..L. .A..S..W. K..................

P.I..N.S ..P.L..G. ..................

.Q....T.. R..L.L..E ................... 


\section{Appendix 2 NTE-like proteins and PROSITE entry}

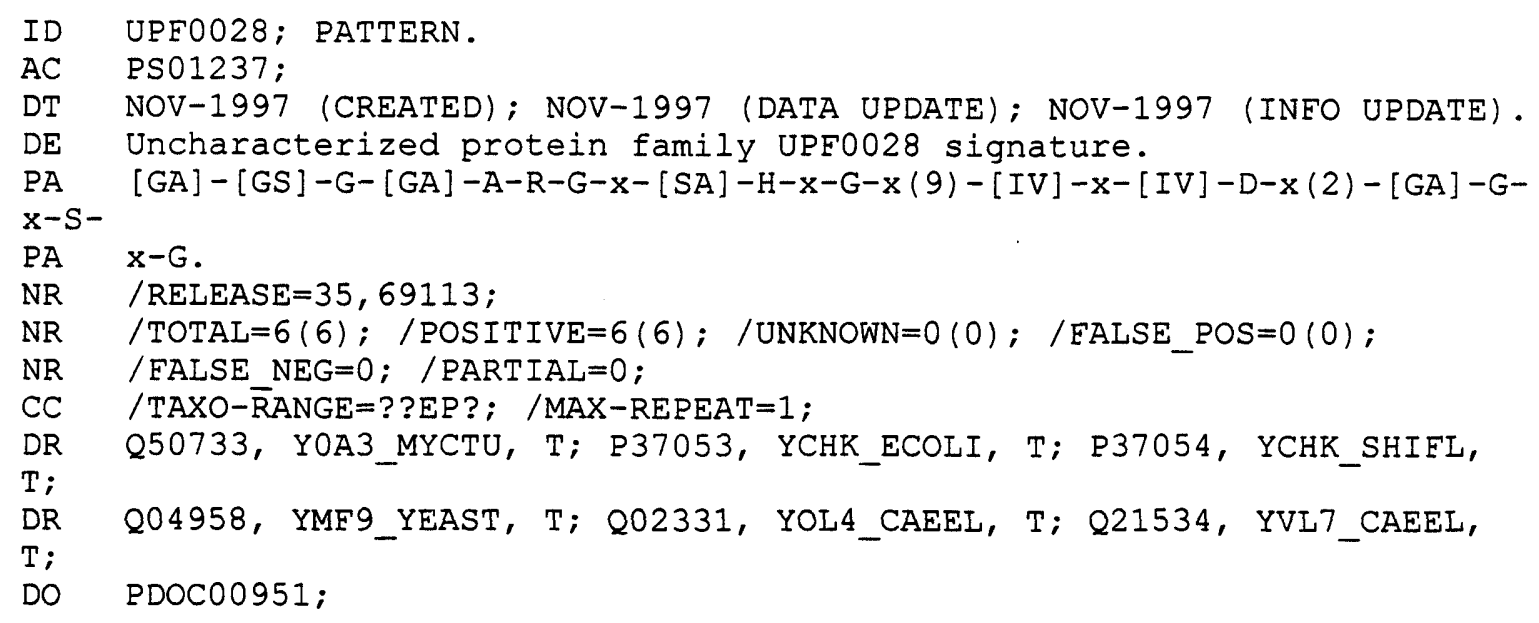

$\{$ PDOC00951\}

$\{P S 01237$; UPF0028\}

\{BEGIN\}

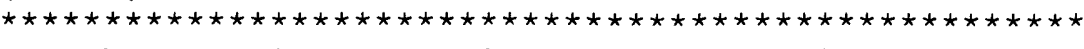

* Uncharacterized protein family UPF0028 signature *

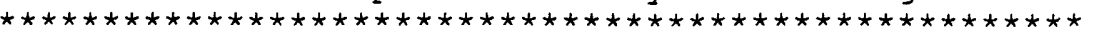

The following uncharacterized proteins have been shown [1] to share regions of similarities:

- Yeast chromosome XIII hypothetical protein YMR059c (1679 aa).

- Caenorhabditis elegans hypothetical protein ZK370.4 (1351 aa).

- Caenorhabditis elegans hypothetical protein M110.7 (880 aa).

- Escherichia coli hypothetical protein ychK (314 aa).

- Mycobacterium tuberculosis hypothetical protein MtCY9C4.03c (583 aa)

These are proteins of variable size which share a domain of about 200

residues. This domain is located at the c-terminal of the eukaryotic members of this family. It can be picked up the database by the

following glycine-rich pattern.

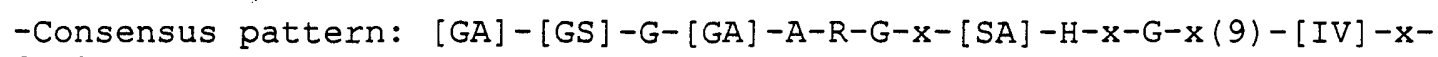

[IV] -D-

$$
x(2)-[G A]-G-x-S-x-G
$$

-Sequences known to belong to this class detected by the pattern: ALI. - Other sequence(s) detected in SWISS-PROT: NONE.

-Last update: November 1997 / First entry.

[ 1] Bairoch A.

Unpublished observations (1997). 


\section{Mtcy20b11}

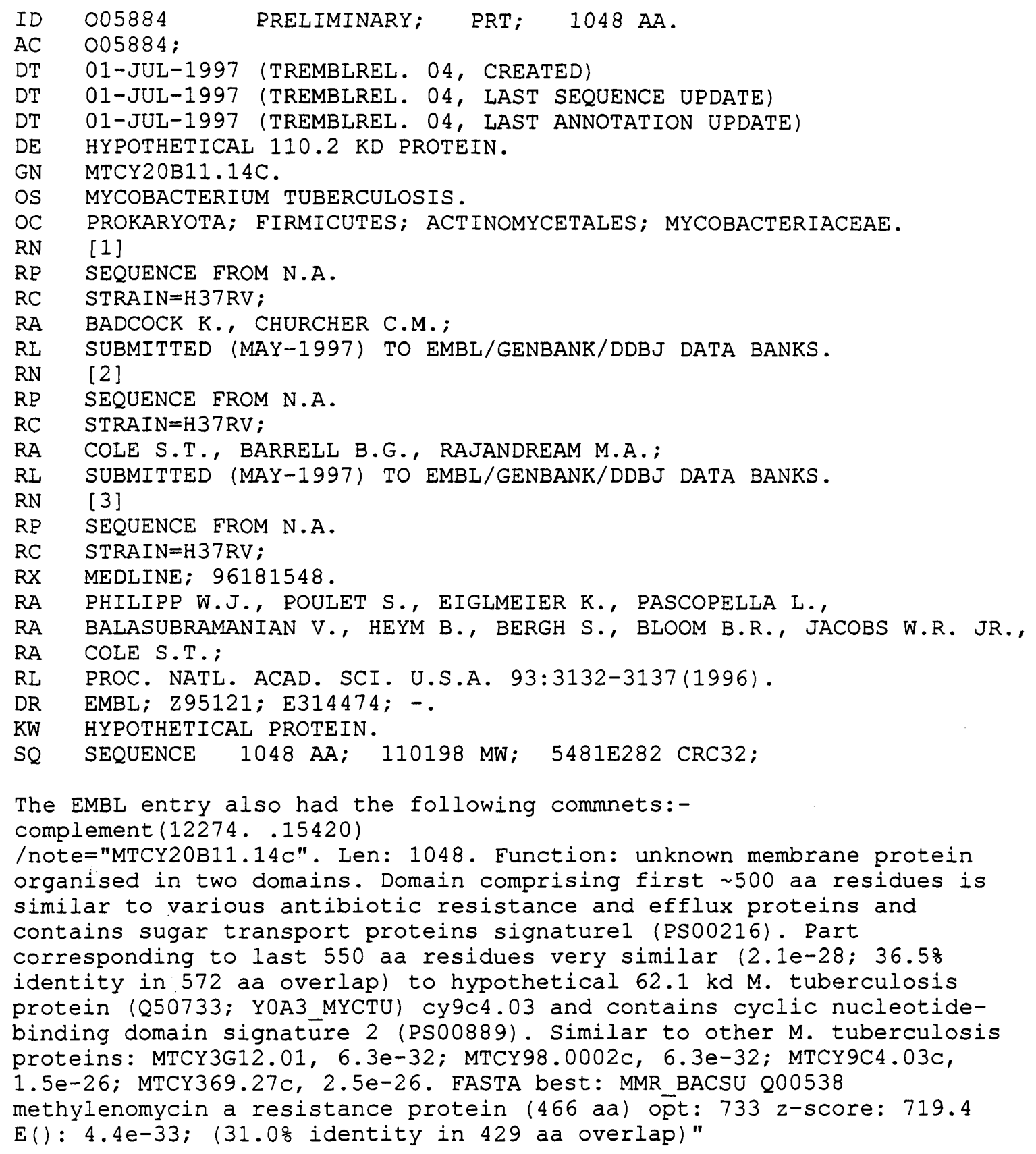

005884 Length: 1048 Eebruary 12, 1998 12:46 Type: P Check: 5529 ..

1 MHISLHGGKG FANLTRRRRP SSASVLLVAG FGAFLAFLDS TIVNIAFPDI

51 QRSFPSYDIG SLSWILNGYN IVFAAFMVAA GRLADLIGRR RTFLSGVLVF

101 TIASGLCAVA GSVEQLVAFR VLQGIGAAIL VPASLALVVE GFDAARRAHA

151 IGLWGAAAAI AAGLGPPIGG LLVEWAGWRW VLLVNVPLGI VAAIATKRML

201 VESRASGRRR MPDLRGALLL AVTLGLVTLG LVKGPDWGWL SVATVGSFLA

251 SVLTSVGFVH SSRSHPAPLV EPALLRSRSE VAGNLLTLVA AAGEYCYGLT

301 HVLYLNYVWH YSLLKAGFAI APAAVVAAVV AAALGRVAGR HGHRVIVLVG

351 ALVWAGSLVW YLQRVGSEPD FLRVWLPGQL LQGIGVGATL PVLSSAALAE

401 VAKGGSYATS SAVVSTTRQL GAVLGVAVMV ILIGKPEHGT AEEALRRGWA

451 MAAICEIAVA VAAAVLGRTN RNPVQMPAPE PAIAPRLEPP IPQPAAAPIE

501 HWAAGDADPL GNLPLFAGLD AATLAQLGEH VEDVELEAGC YLFHEGDPSD

551 SLYVIRTGRV QVLQDSIVLK ELGRGEVLGE LGLLIDAPRS ATVRALRDTK 
601 LVRLTKAQFD EIADHGALAA LVKVLATRLR EAPPPATDST SPEVVVSVIG

651 VSGDAPVPAV AAGLLTALSA RLRAVDPGRV DRDGLDRAER VADKVVLHAA

701 VEDAGWRDFC LRVADRIVLV AGDPNPQAAR LPARARGADI VLAGPAASRE

751 HRRQWEELIT PRSVHVVHYR RILENVRPLA ARIAGRSIGL VLGGGGARGF

801 AHLGVLDELE RVGVTIDRFA GTSMGAVIAV FGACGMDAAT ADAYAYEYFI

851 RHNPLSDYAF PVRGLVRGRR TLTLLEAAFG DRLVEELPKE FRCVSVDLLA

901 RRPVVHRRGR LVDVIGCSLR LPGIYPPQVY NGRLHVDGGV LDNLPVSTRA

951 SPDGPLIAVS IGLGGGGPGS ARQDGSPKVP GIGDTLMRTM TIGSQRGADA

1001 ALSLAQVVIR PDTGAVGLLE FHQIDAAREA GRVAAREAMP HIMALLNR

\section{Q12}

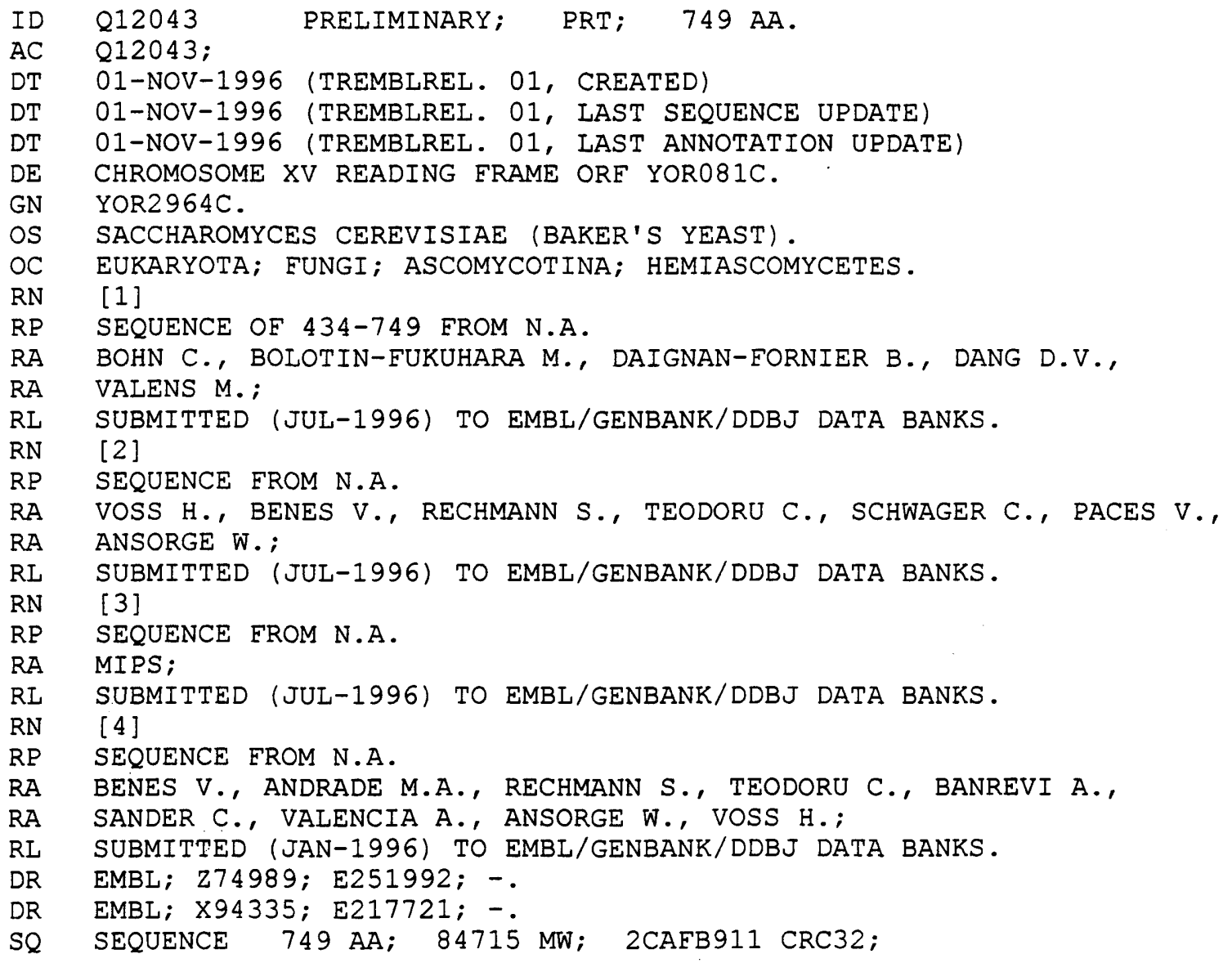

Q12043 Length: 749 February 11, 1998 16:03 Type: P Check: 8887 ..

1 MSNTLPVTEF LLSKYYELSN TPATDSSSLF KWLYHKTLSR KQLLISDLSS

51 QKKHAISYDQ WNDIASRLDD LTGLSEWKTI DESSLYNYKL LQDLTIRMRH

101 LRTTHDYHRL LYLIRTKWVR NLGNMNNVNL YRHSHTGTKQ IIHDYLEESQ

151 AVLTALIHQS NMNDHYLLGI LQQTRRNIGR TALVLSGGST FGLFHIGVLA

201 ALFESDLMPK VISGSSAGAI VASIFCVHTT QEIPSLLTNV LNMEFNIFND

251 DNSKSPNENL LIKISRFCQN GTWFNNQPLI NTMLSFLGNL TFREAYNKTG

301 KILNITVSPA SIYEQPKLLN NLTAPNVLIW SAVCASCSLP GVEPSTPLEE

351 KDPHTGKIKE WGATNLHLSN MKFMDGSVDN DMPISRLSEM FNVDHIIACQ

401 VNIHVFPLLK FSNTCVGGEI EKEITARFRN QVTKIFKFFS DETIHFLDIL

451 KELEFHPYLM TKLKHLFLQQ YSGNVTILPD LSMVGQFHEV LKNPSQLFLL

501 HQTTLGARAT WPKISMIQNN CGQEFALDKA ITELKEKIII SSSIKNPLQF

551 YQPRESEQIK SLSIMDADLP GVDLEESSSN SLSIIKSPNK TAAPGREPLQ

601 PLPSPSSTFN KRKMDMLSPS PSPSTSPQRS KSSFTQQGTR QKANSLSFAI

651 GASSLRLKKS PLKVPSRPQF KKRSSYYNQN MSAEMRKNRK KSGTISSYDV

701 QTNSEDFPIP AIENGSFDNT LFNPSREPMD AMSAATNDNE MNNSDIFQN 


\section{SWISSCHEESE}

Currently no peptide sequence for swisscheese has been submitted any of the sequence databases, this 'database entry' consists of the header for the EMBL DNA entry and a translation based on the published paper.

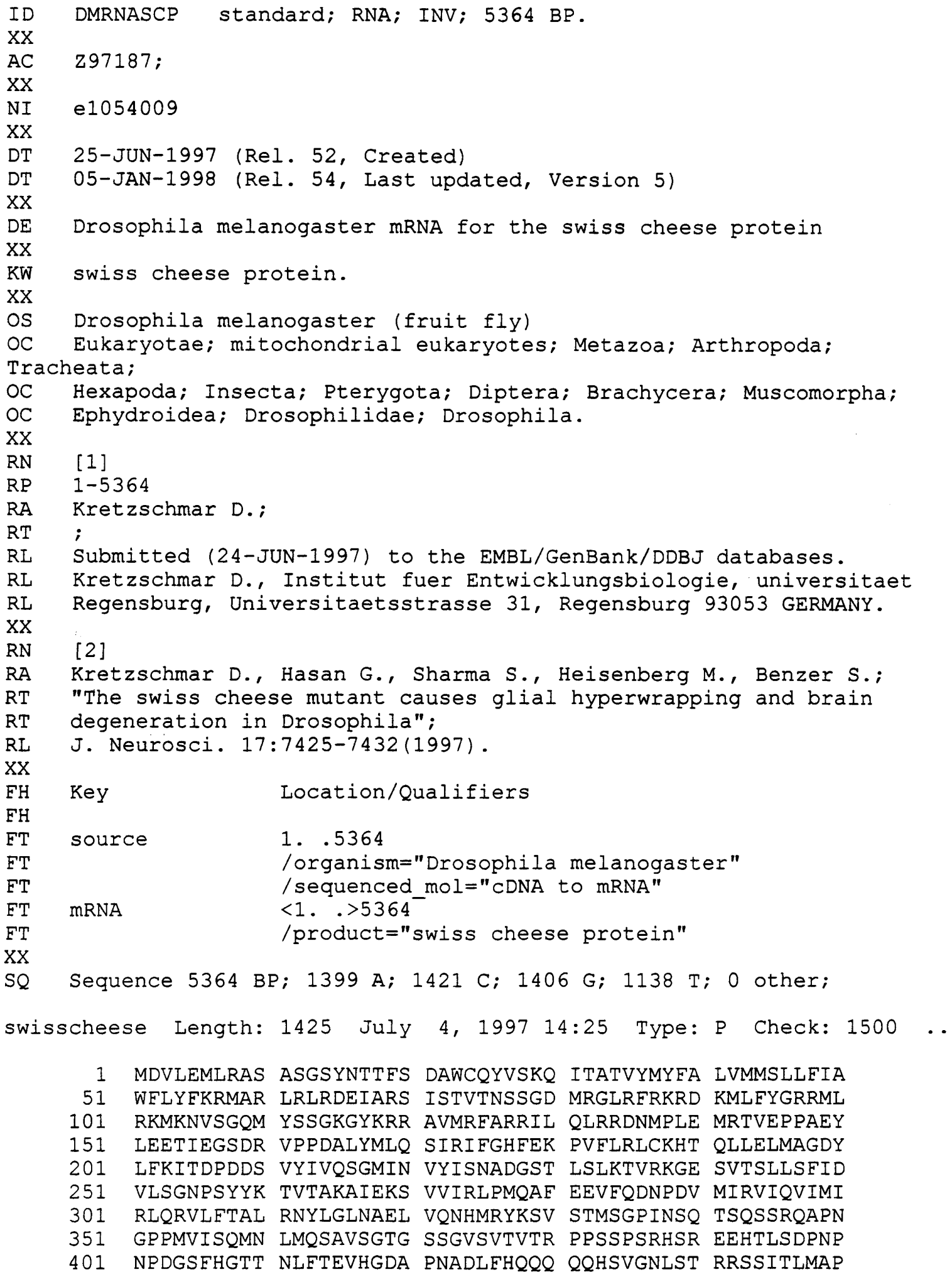


451 DPSHSCLQTP GVTTSIDMRL VQSSAVDSLR KELGLSEEDS HIIEPFVELR

501 ELEPNVTLIT EGNADDVCVW FVMTGTLAVY QSNQDATRAK QDKSDMLIHF

551 VHPGEIVGGL AMLTGEASAY TIRSRSITRI AFIRRAAIYQ IMRQRPRIVL

601 DLGNGVVRRL SPIVRQCDYA LDWIFLESGR AVYRQDESSD STYIVLSGRM

651 RSVITHPGGK KEIVGEYGKG DLVGIVEMIT ETSRTTTVMA VRDSELAKLP

701 EGLENAIKLR YPIVVTKLIS FLSHRELGSM QTRSGSGAPG APVEANPVTH

751 KYSTVALVPI TDEVPMTPFT YELYHSLCAI GPVLHLTSDV VRKQLGSNIF

801 EAANEYRLTS WLAQQEDRNI ITLYQCDSSL SAWTQRCMRQ ADVILIVGLG

851 DRSHLVGKFE REIDRLAMRT QKELVLLYPE ASNAKPANTL SWLNARPWVT

901 KHHHVLCVKR IETRKSQYRI NDLYSRVLLS EPNMHSDFSR LARWLTGNSI

951 GLVLGGGGAR GAAHIGMLKA IQEAGIPVDM VGGVSIGALM GALWCSERNI

1001 TTVTQKAREW SKKMTKWFLQ LLDLTYPITS MFSGREFNKT IHDTFGDVSI

1051 EDLWIPYFTL TTDITASCHR IHTNGSIWRY VRSSMSLSGY MPPLCDPKDG

1101 HLLLDGGYVN NLPADVMHNL GAAHIIAIDV GSQDDTDLTN YGDDLSGWWL

1151 LYKKWNPFTS PVKVPDLPDI QSRLAYVSCV RQLEEVKNSD YCEYIRPPID

1201 KYKTLAFGSF DEIRDVGYVF GKNYEESMAK AGRLGRENQW FNKEPPKRVN

1251 HASLNEYTEI DLAQIVCRLP ETYAVNTAEL FSEDEDCDGY ISEPTTLNTD

1301 RRRIQVSRAG NSLSFSETEM DSDVELDLKL ERKTDKSTQS SPPSNSRSDM

1351 RGKEEARHMS NWHWGVKHKD ETGSGANEAT KTQTGQEQEL QQEQQDQGAT

1401 AEQLVDKDKE ENKENRSSPN NETKN

\section{YOA3 MYCTU}

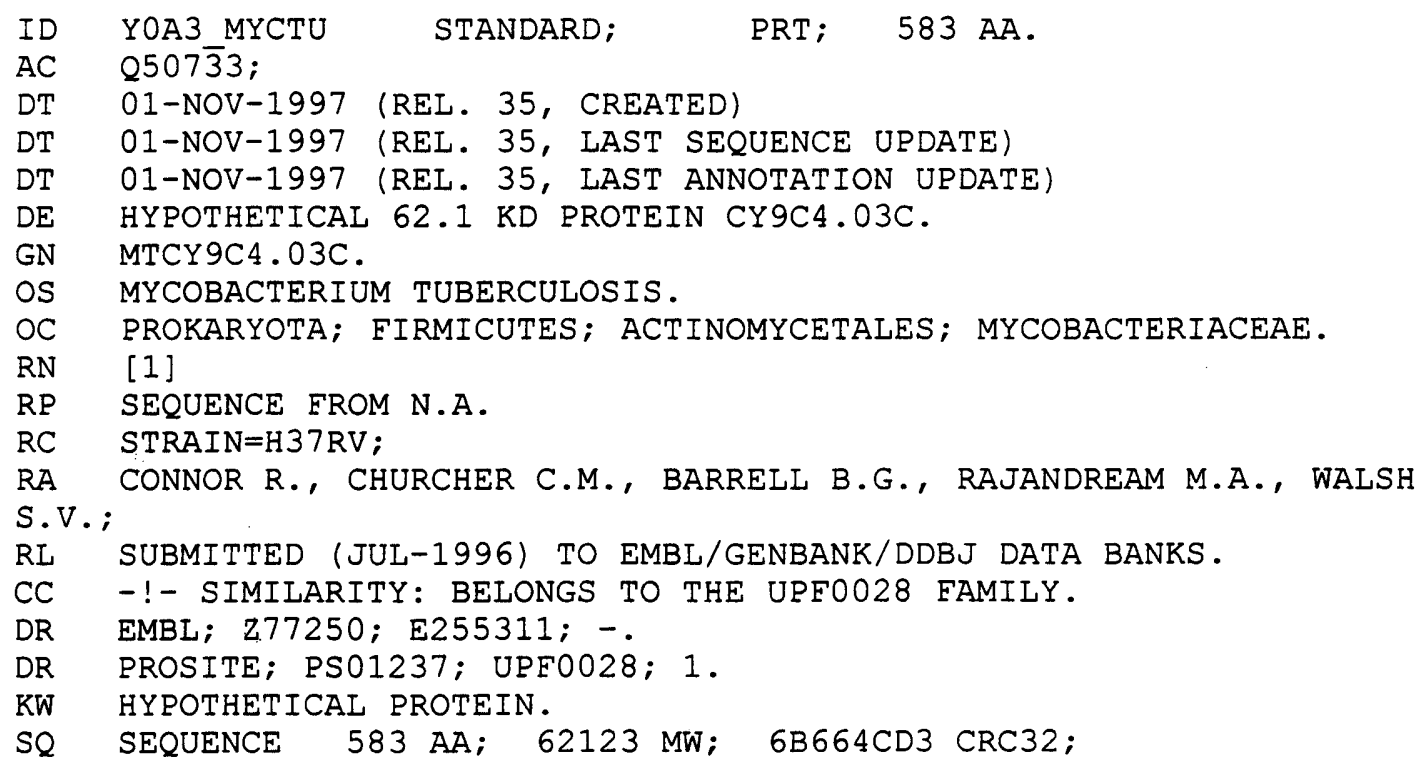

YOA3_MYCTU Length: 583 February 11, 1998 15:57 Type: P Check: 216 . 


\section{YCHK ECOII}

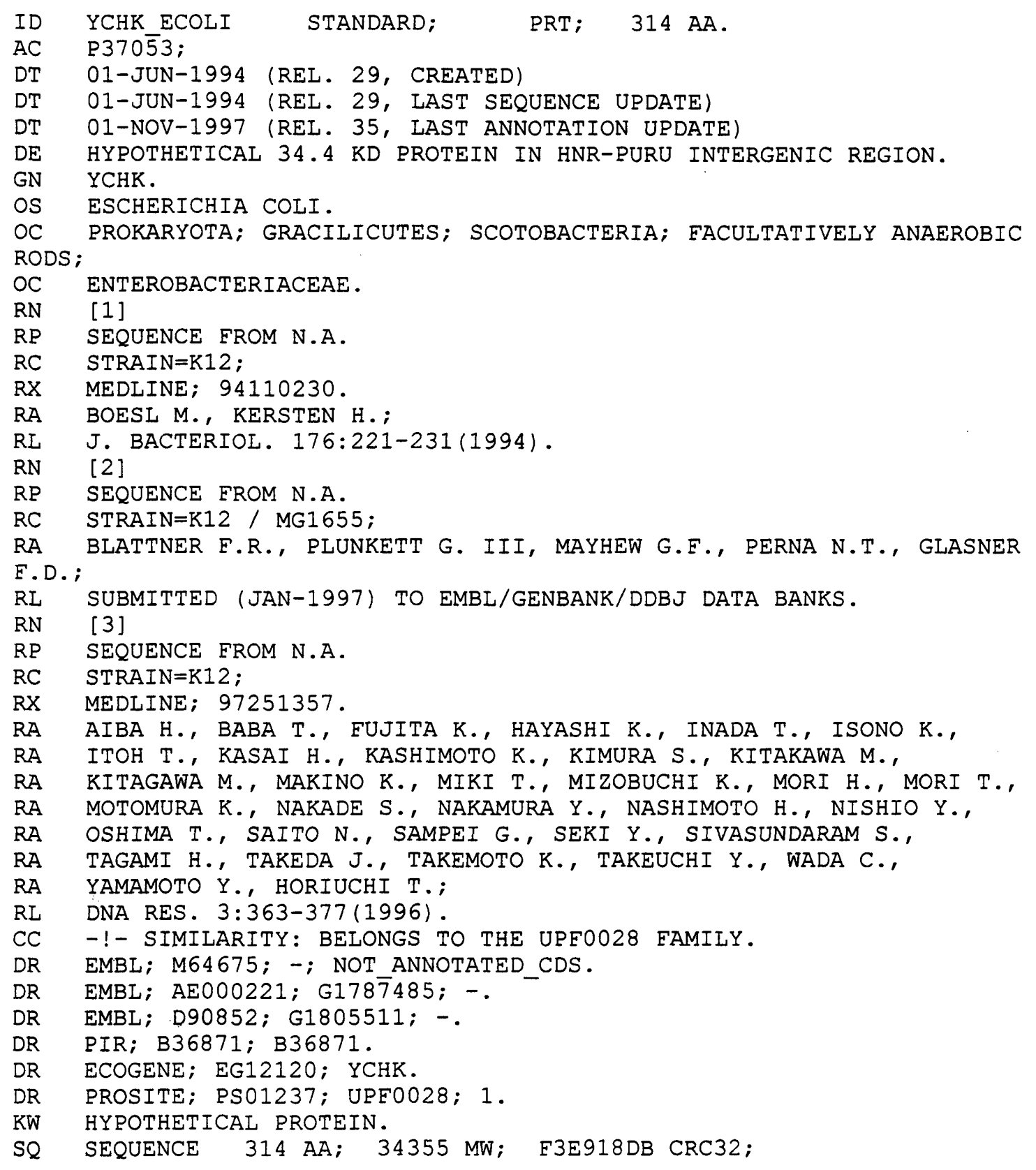

YCHK_ECOLI Length: 314 Eebruary 11, 1998 15:56 Type: P Check: 307 $\cdots$

1 MATIAEQGNL AGIMRKIKIG LALGSGAARG WSHIGVINAL KKVGIEIDIV

51 AGCSIGSLVG AAYACDRLSA LEDWVTSFSY WDVLRLMDLS WQRGGLLRGE

101 RVENQYREIM PETEIENCSR REAAVATNLS TGRELWFTEG DLHLAIRASC

151 SIPGLMAPVA HNGYWLVDGA VVNPIPISLT RALGADIVIA VDLQHDAHLM

201 QQDLISFNVS EENSENGDSL PWHARLKERL GSITTRRAVT APTATEIMTT

251 SIQVLENRLK RNRMAGDPPD ILIQPVCPQI STLDFHRAHA AIAAGQLAVE

301 RKMDELLPLV RTNI 


\section{YCHK_SHIFI}

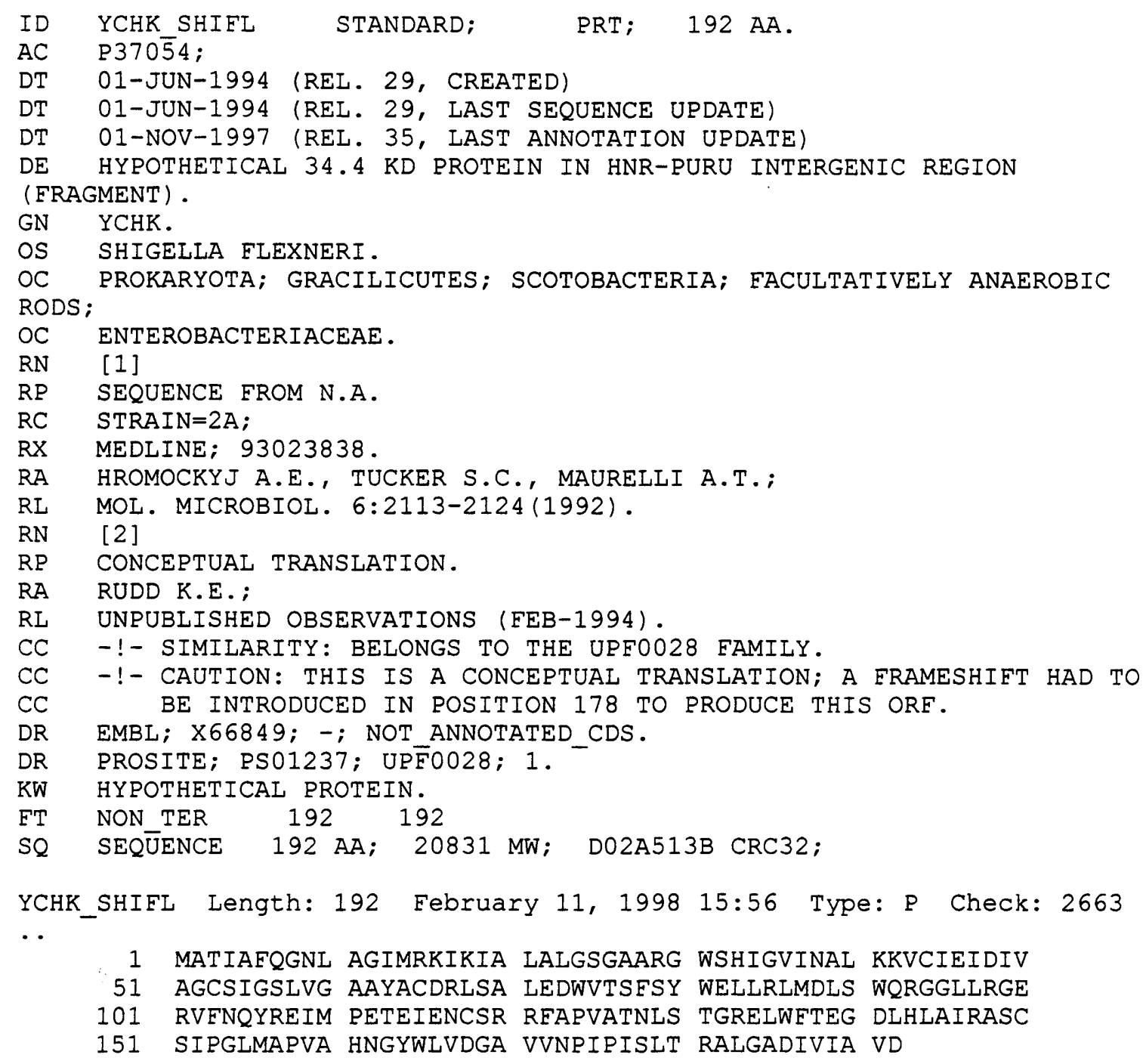

\section{YK69_YEAST}

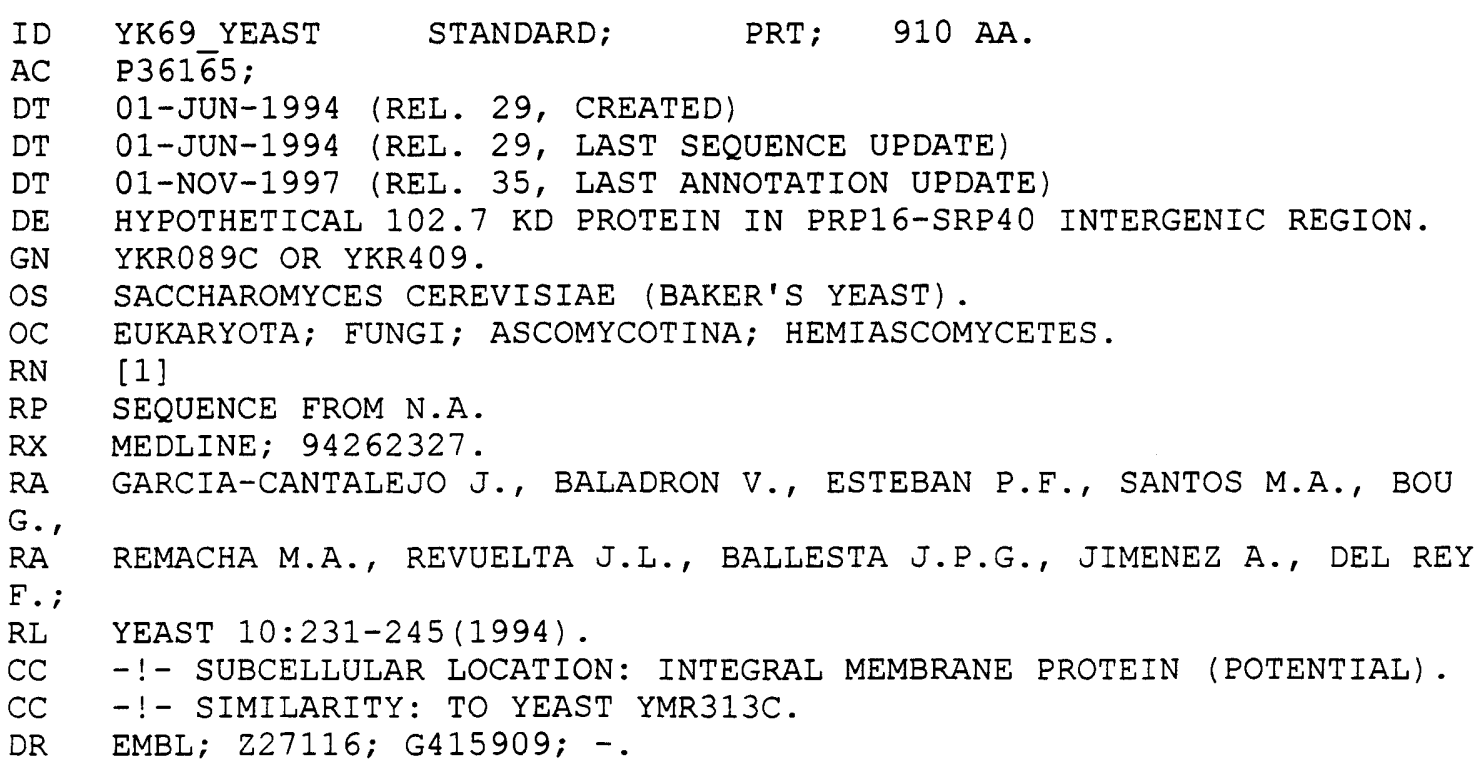




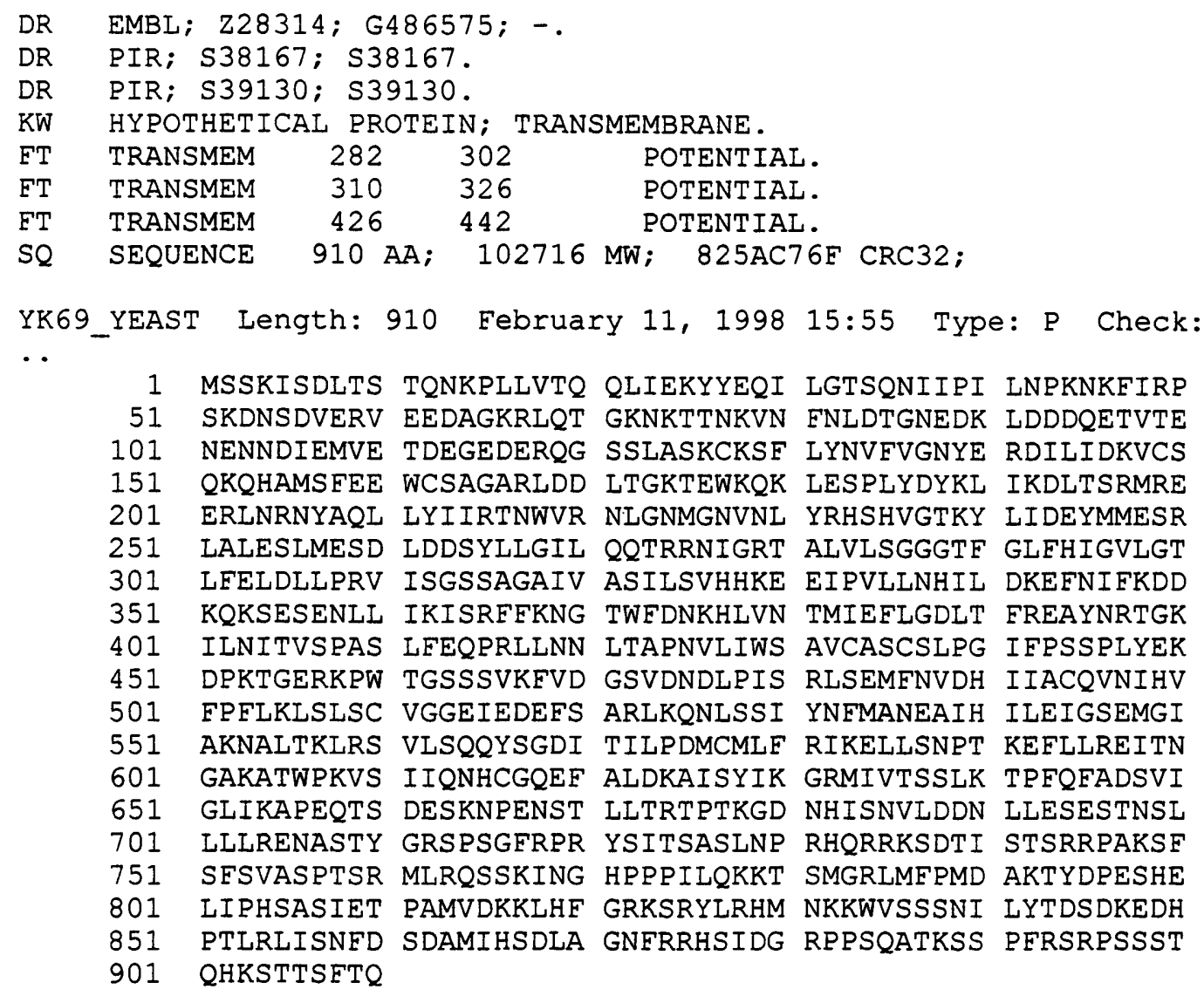

\section{YIBK}

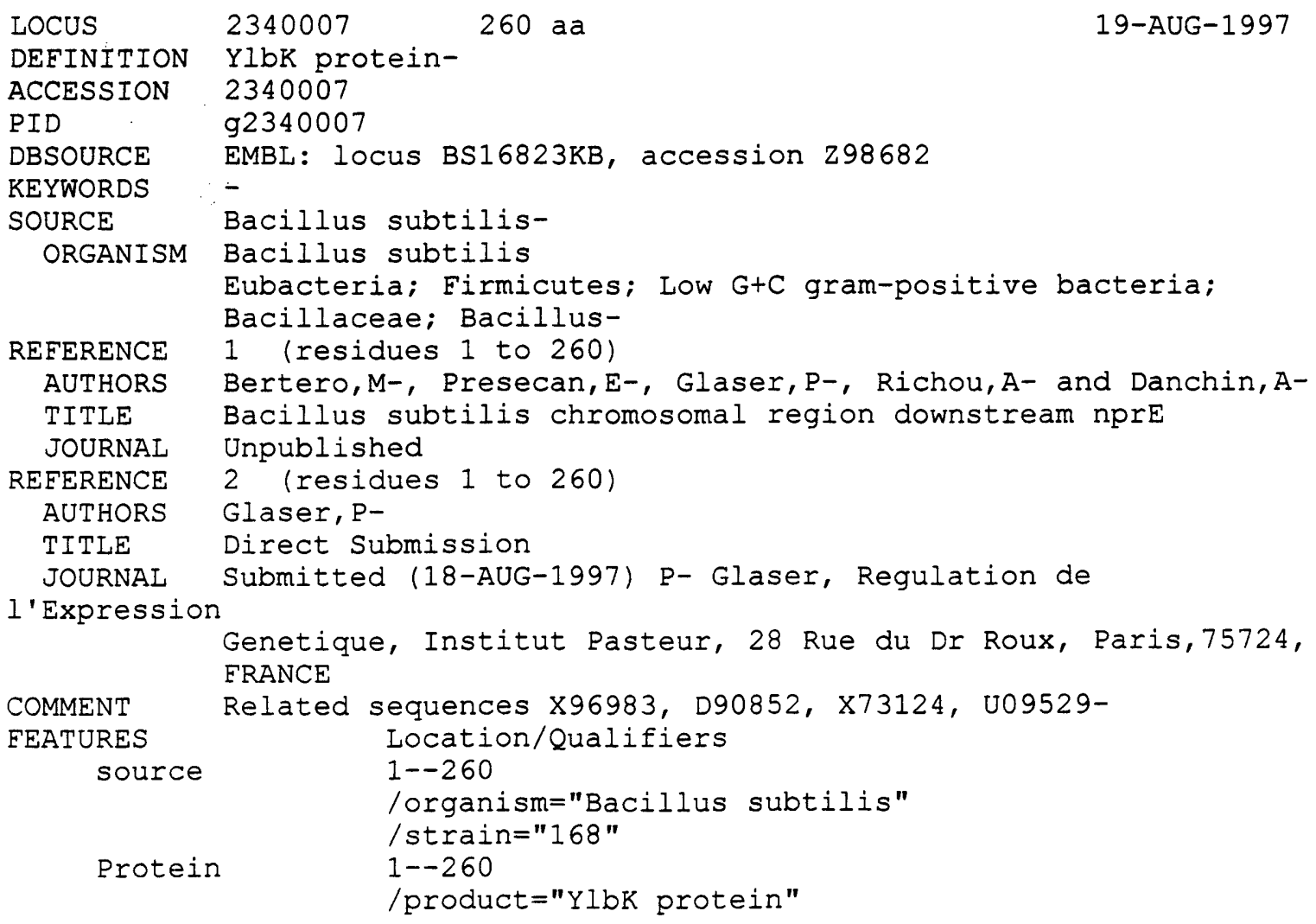




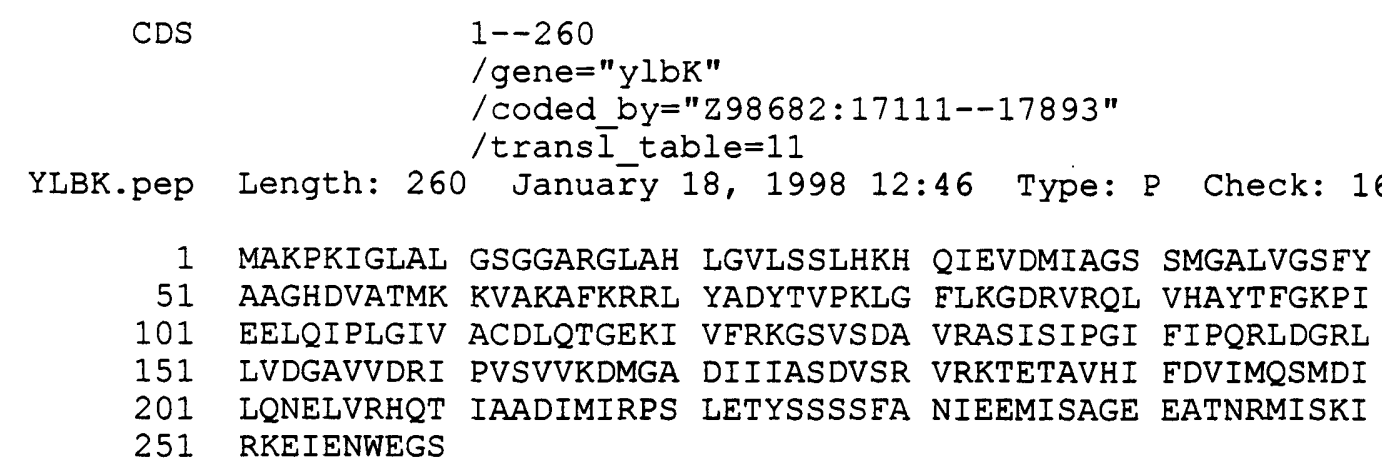

\section{YMF9 YEAST}

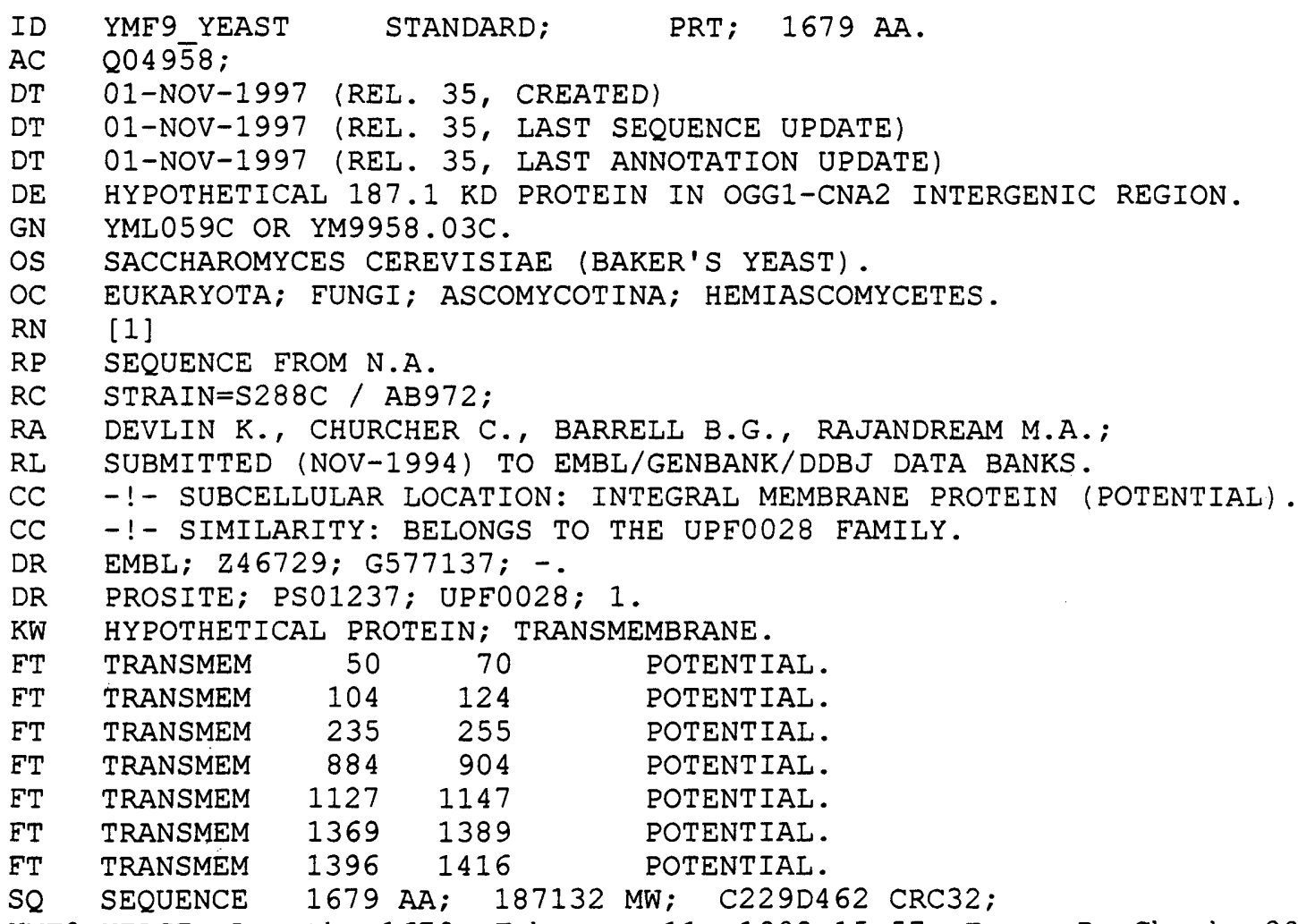
YMF9_YEAST Length: 1679 February 11, 1998 15:57 Type: P Check: 209 $\cdots$

1 MRSMNCTTNN TNNTGQNTKN SLGSSFNSSN YTSYRFQTCL TDQIISEAQT

51 WSLSSLFNES WVVSYFVMGA SRMIFRYGWY LATLSLLRIP KWIFFKLHHV 101 QFTLSFWLIL FALAVIVFVT YTIMKERILS QYKRLTPEFL PLENTGKSGS 151 SANINAASTO SANAPPAIGS STTGASSIID SKKHSLKDGN ENETFLSSYL 201 DQFLSAIKIF GYLEKPVFHD LTKNMKTQKM DEGEILLLDS TIGFAIVVEG 251 TLQLYHEVDH SDKDHGDETD HSDTDGLDDQ DRDEEDEEED DDIDNYDTKS 301 CSSNLIDEED ESVGYIHLKN GLGNEQLLNT VKPGNPLTSL VSILNLFTHS 351 MSSYGNSNEP SELSSPIDTT VSVNNMFCSS EQNFSNTDSM TNSTNSFPTF 401 PSSMPKLVAR AATDCTIGII PPQSFAKLTA KYPRSASHII QMVLTKLYHV 451 TFQTAHDYLG LTKEIMDIEV LLNKSIVYEL PYYLKEAVIR KFKTVDKSSG 501 SADLEPKPKN SNASSKLKKP PKAKPSDGII QSLKIANANA NTSSNSLSLK 551 PEFTHHPSSR HVVLGSRDQF NPGDLLSNVP LSRTMDILSP NPIHNNNRNK 601 SNGINTSTSN QHKRSSRSSS NNASVHSKKF SSLSPELRNA QLSTSPLSLD 651 NTSVHDHIHP SPVHLKGRVS PRPNLLPTTS FSAAQEETED SALRMALVEA 701 MLTYLGVNKS NMSVSSSSIA NMSSLNSPQL NEMYSRRPSN ASFLMSPHCT 751 PSDISVASSF ASPQTQPTML RILPKEYTIS NKRHNKSKSQ DKKKPRAYKE 801 ELTPNLDFED VKKDFAQGIQ LKFFKKGTTI VEQNARGKGL FYIISGKVNV 851 TTNSSSSVVS SMSKPEQVSA QSSHKGENPH HTQHLLYSVG SGGIVGYLSS 
901 LIGYKSFVNI VAKSDVYVGF LSSATLERLF DKYFLIYLRI SDSLTKLLSS

951 RLLKLDHALE WVHLRASETL FSQGDSANGI YVVLNGRLRQ LQQQSLSNSN

1001 TSSEEVETQN IILGELAQGE SFGEVEVLTA MNRYSTIVAV RDSELARIPR

1051 TLEELLALEH PSIMIRVSRL VAKKIVGDRT VPALTGDPLS IKENDFTSLI

1101 PPTKASYSSS LSHKPQNITS GTITFRTITI LPITSGLPVE AFAMKLVQAE

1151 KQVGRTTIGL NQRTTLTHLG RHAFDRLSKL KQSGYFAELE EMYQTVVYIS

1201 DTPVKSNWTR TCIAQGDCIL LLADARSPSA EIGEYEKLIL NSKTTARTEL

1251 ILLHPERYVE PGLTHKWLRY RPWVHSHHHI QFSLTGTTLM NEGKMHVLNN

1301 GALALMDKLI QTEFSRKTQQ NISKLLPDSI KNTVENFSSR FMKSKRQYYT

1351 PVHRHKNDEL RLARILSGQA IGLVLGGGGA RGISHLGVIQ AIEEQGIPVD

1401 VIGGTSIGSF VGGLYAKDYD LVPIYGRVKK FAGRISSIWR MLTDLTWPVT

1451 SYTTGHEFNR GIWKTFGDTR IEDEWIQYYC NSTNITDSVQ EIHSFGYAWR

1501 YIRASMSLAG LLPPLEENGS MLLDGGYVDN LPVTEMRARG CQTIFAVDVG

1551 SADDRTPMEY GDSLNGFWII FNRWNPFSSH PNIPNMAEIQ VRLGYVASVN

1601 ALEKAKNTPG VVYVRPPIEE YATLDFSKFE EIYHVGVDYG RIFLQGLIDD

1651 DKMPYIPGSQ ETTLNSQVPE FLLHRRNSI

\section{YOL4_CAEEL}

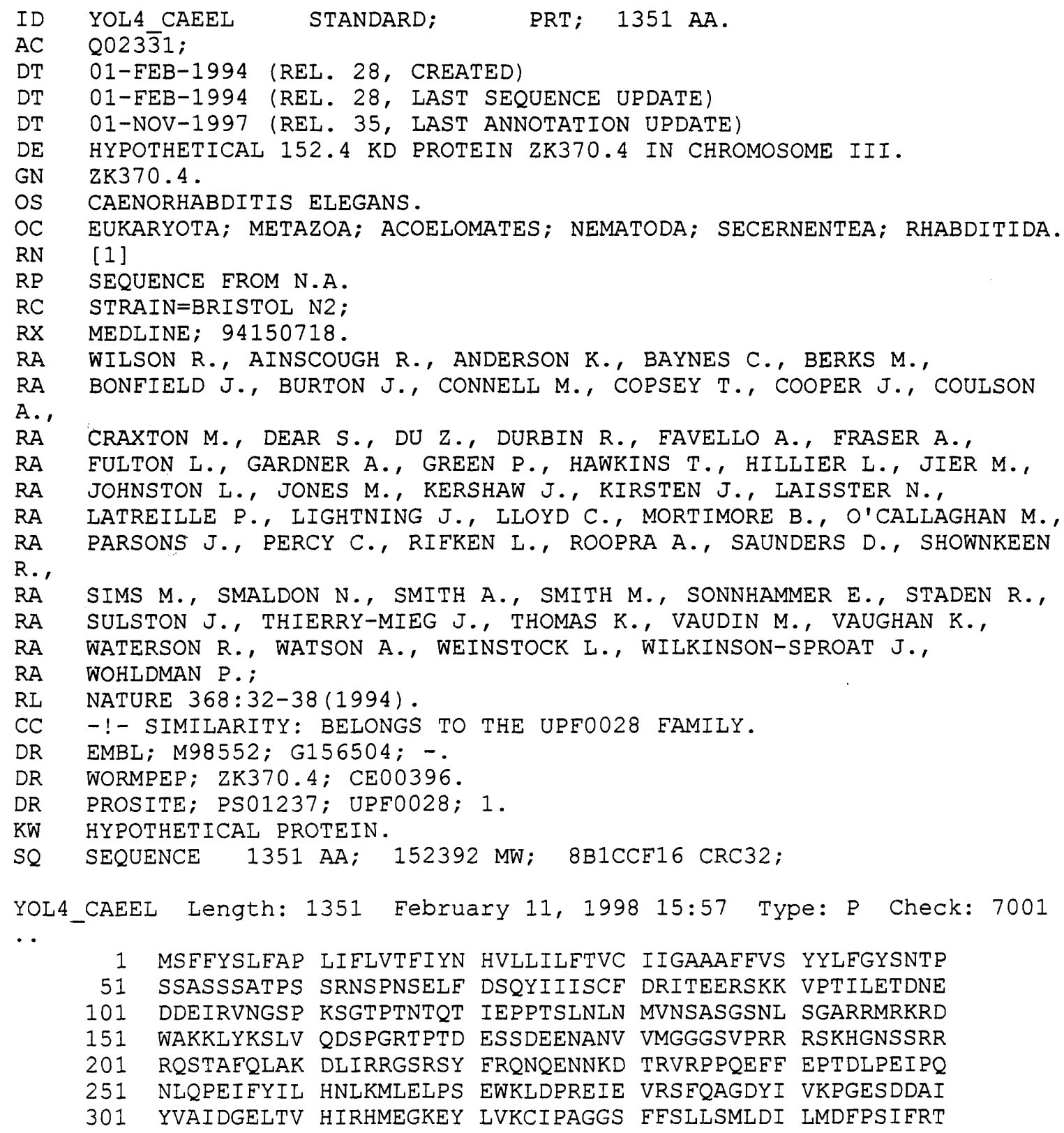


351 VALKAADPCR VAKFPITSER ESYHKNPEAW IRPIQIVITR LLHVTLTTLH 401 QYMGLSSELM RRFTLPRFFF EKRKKISKFL VAYLFSYEQD QLIVARKWMA

451 EAFGLVADDV VSEQIGSKIH LESYEAGHVI IEQGAEEEVL MMVLHGNLIL

501 AQESLFDEEN NEEDESAVIR VTARELVGGL QILTNEPSFY TIRAAVQTRV

551 AIMKKKDFSA FLEAHPEIYL PVAHSVLRRL SPFLRGVDFA LDWVLVDSGH

601 ACYRAGDMAD SLEVVLSGRL RSVEKKTVVE EFGRGDVLGM MEVLTKKPRA

651 TTVLAVRFSQ LARVPEGLLN FIKMQYPQVG FRLVQLLGQY YSQTNRRAPF

701 AAPTVIRTNE LGATDPMSHI KNLHTIAVVP ASPDVPLVPF TCELYHALSS

751 NLRVLRLSSQ KVAACLDPSV LEKQADFRLM HWLNVQEDTY PLVIYECDFT

801 QTNWTRRCLR QADAILVVAI GGKTPEKQTL MRELMNMNQD GVRTNKELIL

851 LWPESTKTPS GTIEWLKNSY FSGHHHIRAP KRMLQWNRKE RKMSRAEVMT

901 PTSVENEVIE YYEKNVFWTP DRRSDFSRLA RILTGNAIGL VLGGGGARGA

951 AHVGVLRALR EEGIPVDIVG GTSIGSLIGG LYAETPDDVV VETRAASWEN

1001 GMSSLWRKLI DLTYAHSAMF TGAQFNFSIK DLFEERLIED LWISYFCIST

1051 DISTSEMRVH RSGPLWAYCR ASMSLAGYLP PLCDPQDGHI LLDGGYVNNV

1101 PADVMRNLGA RCVIACDVGS IEETNLYDYG DSLSGMWVLL KRLNPFGTPP

1151 RILNMEEIQS RLAYVSCVRQ LEVVKKASYC RYLRPPIEPE KTLDFPKEQE

1201 IMELGLKYGR EACHELITND RAGILGDEKE TRKFKRQQSR REKPDVSRAV

1251 SFTDLAAAMS KIPVVRPTLR HSMSLNPSAN GPVGRAGDHF LLDDDLFNDT

1301 DYYEEESSQS ENGNESETED EDLVIGPPSS SSSGGNVSEN PRGTTPRPPS

$1351 \mathrm{~S}$

\section{YVL7_CAEEL}

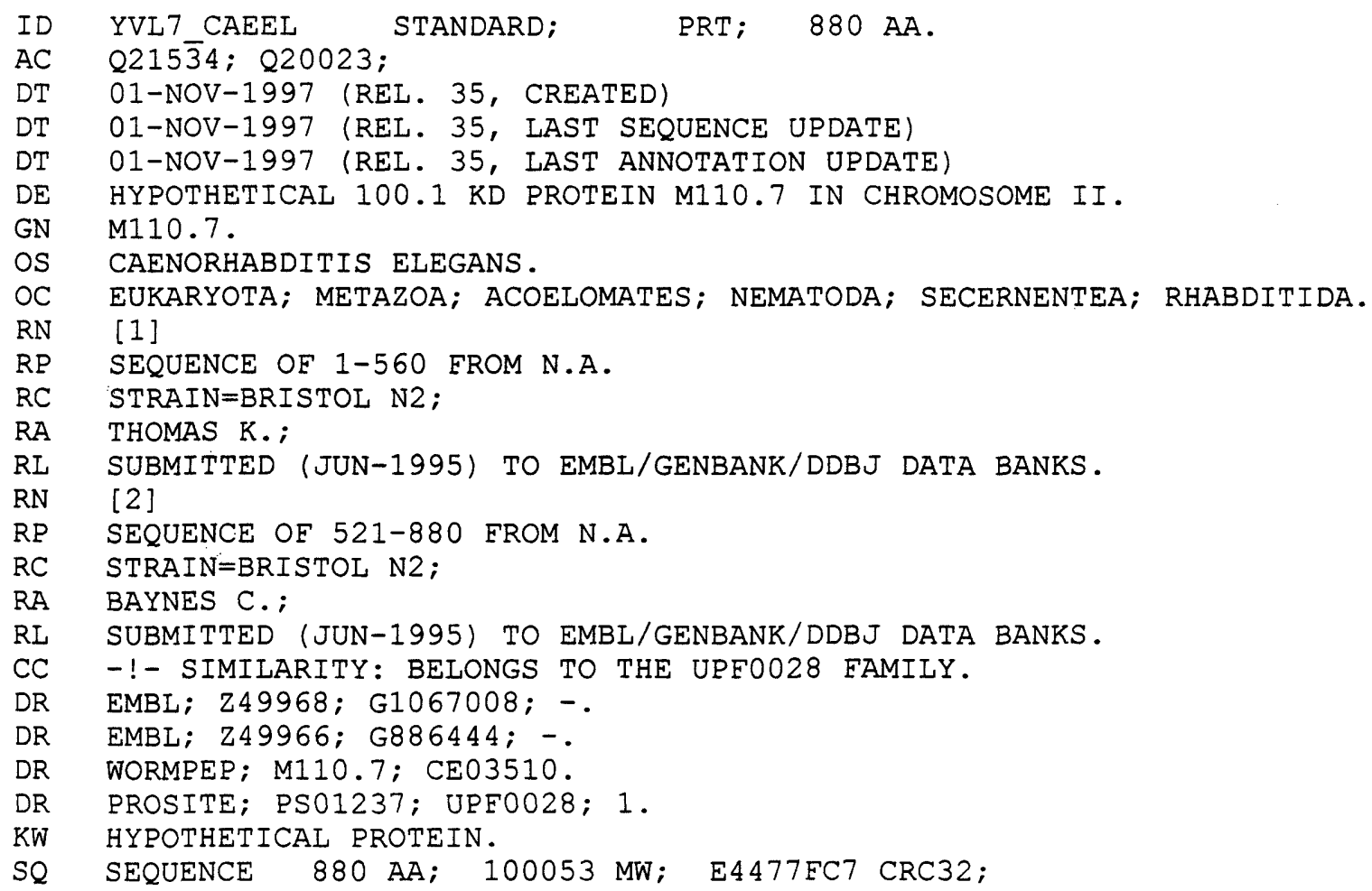

YVL7_CAEEL Length: 880 February 11, 1998 15:57 Type: P Check: 2638 $\cdots$

1 MTPDKKRDSS EKISKQPPRE LFEPNEQEQV PSHIKPEIEF VLKALEGLEL

51 PTTWQLDPKD VETLSIDTGS VVLSPGRAND VIVVVISGEL GIFTNVSLNE

101 KPNNKYIHLK ALTSCRVATY HLTSFHTSFI ANPQQWIRTI QVVMTRLQQC

151 TLITCNMYLG IGGKCLNAGD LEHDDTGTSL TFDVYPGDML PSMQILTNEP

201 AMCSAKALEK TIYFKICRDE YIQFLFAHPV IYLRLAFHAL QFISPFARVF

251 DMAVHWHRIE TGQALFRQGD KSDSMHIVMG GRLRAVDSTK IIEEYGRLDL

301 IGITDMAEKR PRRNTVMAVR ESHIVCIPEN LLSEVKIRYP QVNSARWSRR

351 DFTEFSASRR VPLTEFTCEL YNQLSKHVKT LRLSSSVVEN YFESEVITKK

401 ADYGLMHWLN VQEIAYSLVL YQCDFHKTNW TRRCLRMADA ILMVALGTES 
451 KEEQVLAEAL LSCNEKGVRQ SKELVFLWPI DTPTPSGTAA WIKESYYSGY

501 HHLRAPNRLF SFPLKTREKK IVEYYETTVY GEISYQSDFS RLARILTGNA

551 IGIVEGGGGA RGAAHAGALR ALIEKKVQID MVGGTSIGAL FGSLYATTPD

601 IRAVGRMKDF FTDRLRNNIL DVVRDLTWPY CGILTGHRFN LCVQRMLNDV

651 NIEDCWVSFE CITTDLTSSS MRIHRNGIMW PVVRSSMSIA GYVPPICDPQ

701 DGHLLLDGAY VNNLPADIMR SLGANVVIAI DVGMSDDNTN LRNYGFSISG

751 TWCLFKRWWP FGEELRVINM NEVQNRLAYV CCVNQMEIVK NAQYCYYVKL

801 PIESFGIFDF SKFDQAAQIG YDITKQKMEE FFEDSVATRR KLLGCARNVR

851 QTPQKSKNDN ILSFVNMPVL PKPPSDIKSD 


\title{
Appendix 3 Gapped BLAST search of Swissprot
}

\author{
This search used NTE1 to search SWISSPROT, it misses some of the \\ sequences known to contain an NTE like esterase domain as they had not yet \\ entered the SWISSPROT database.
}

\author{
BLAST Search Results \\ Commencing search, please wait for results.
} BLASTP 2.0.3 [NOV-14-1997]

Reference: Altschul, Stephen F., Thomas L. Madden, Alejandro A.

Sch\&auml;ffer, Jinghui Zhang, zheng Zhang, Webb Miller, and David J. Lipman (1997), "Gapped BLAST and PSI-BLAST: a new generation of protein database search programs", Nucleic Acids Res. 25:3389-3402.

Query $=$ NTE (1327 letters)

Database: Non-redundant SwissProt sequences

68,512 sequences; $24,689,586$ total letters

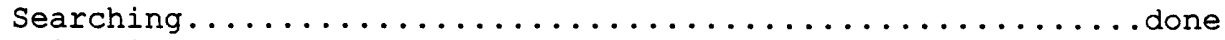

Distribution of 100 Blast Hits on the Query Sequence

Sequences producing significant alignments:

Score

(bits) value

Q02331/YOL4_CAEEL HYPOTHETICAL 152.4 KD PROTEIN ZK370.4 IN C... 680 0.0 Q21534/YVL7 CAEEL HYPOTHETICAL 100.1 KD PROTEIN M110.7 IN CH... 503 e-141 Q04958|YMF9-YEAST HYPOTHETICAL 187.1 KD PROTEIN IN OGG1-CNA2 .. 452 e-126 Q50733/YOA3 MYCTU HYPOTHETICAL 62.1 KD PROTEIN CY9C4.03C 119 2e-26 P37053/YCHK_ECOLI HYPOTHETICAL 34.4 KD PROTEIN IN HNR-PURU I... 103 2e-21 P37054/YCHK SHIFL HYPOTHETICAL 34.4 KD PROTEIN IN HNR-PURU I... 101 8e-21 P05987|KAPR_DICDI CAMP-DEPENDENT PROTEIN KINASE REGULATORY C... 57 le-07 P31319/KAPR APLCA CAMP-DEPENDENT PROTEIN KINASE REGULATORY C... 53 3e-06 P07278|KAPR YEAST CAMP-DEPENDENT PROTEIN KINASE REGULATORY C... 52 6e-06 P31321|KAP1_HUMAN CAMP-DEPENDENT PROTEIN KINASE TYPE I-BETA ... 51 1e-05 P09456|KAPO RAT CAMP-DEPENDENT PROTEIN KINASE TYPE I-ALPHA R.. 50 3e-05 P00514|KAPO_BOVIN CAMP-DEPENDENT PROTEIN KINASE TYPE I-ALPHA... 50 3e-05 P49605/KAPR USTMA CAMP-DEPENDENT PROTEIN KINASE TYPE II REGU. . 49 4e-05 P10644/KAPO_HUMAN CAMP-DEPENDENT PROTEIN KINASE TYPE I-ALPHA... 48 7e-05 P16905/KAPR DROME CAMP-DEPENDENT PROTEIN KINASE REGULATORY C... 48 7e-05 P31322/KAP3_BOVIN AMP-DEPENDENT PROTEIN KINASE TYPE II-BETA... 47 2e-04 P07802/KAPO-PIG CAMP-DEPENDENT PROTEIN KINASE TYPE I-ALPHA R. 47 2e-04 P12849|KAP1-MOUSE AMP-DEPENDENT PROTEIN KINASE TYPE I-BETA $\ldots . .4742$ e-04 P47200|FNRA PSEST TRANSCRIPTIONAL ACTIVATOR PROTEIN FNRA $\cdots 46$ 4e-04 P30625|KAPR CAEEL CAMP-DEPENDENT PROTEIN KINASE REGULATORY C... 46 5e-04 P31323|KAP3 HUMAN CAMP-DEPENDENT PROTEIN KINASE TYPE II-BETA... 46 5e-04 P23926|ANR PSEAE TRANSCRIPTIONAL ACTIVATOR PROTEIN ANR P12369|KAP 3 RAT CAMP-DEPENDENT PROTEIN KINASE TYPE II-BETA R. P37428|FNR SALTY FUMARATE AND NITRATE REDUCTION REGULATORY P.. P03019/FNR_ECOLI FUMARATE AND NITRATE REDUCTION REGULATORY P.. Q90980 $\mathrm{CNG} \overline{3}$ CHICK CYCLIC NUCLEOTIDE GATED CHANNEL, ROD PHOTO... P45199|FNR_HAEIN FUMARATE AND NITRATE REDUCTION REGULATORY P.. Q50734/YOA 4 MYCTU HYPOTHETICAL ABC TRANSPORTER ATP-BINDING P... Q03041/CNG2 BOVIN CYCLIC-NUCLEOTIDE-GATED OLFACTORY CHANNEL ... Q00195/CNG2-RAT CYCLIC-NUCLEOTIDE-GATED OLFACTORY CHANNEL (C. Q28718/CNG2 RABIT CYCLIC-NUCLEOTIDE-GATED OLFACTORY CHANNEL ... P31320|KAPR BLAEM CAMP-DEPENDENT PROTEIN KINASE REGULATORY C... Q24278/CNG DROME CYCLIC-NUCLEOTIDE-GATED CATION CHANNEL (CNG.. Q29441/CNG'3_BOVIN CYCLIC-NUCLEOTIDE-GATED CATION CHANNEL $31 \ldots$ P13861|KAP2 HUMAN CAMP-DEPENDENT PROTEIN KINASE TYPE II-ALPH... Q90805/CNG1_CHICK CYCLIC NUCLEOTIDE GATED CHANNEL, CONE PHOT... Q62398/CNG2 MOUSE CYCLIC-NUCLEOTIDE-GATED OLFACTORY CHANNEL ... P29973/CNG1_HUMAN CGMP-GATED CATION CHANNEL PROTEIN (CYCLIC ... O00194 ICNG1-BOVIN CGMP-GATED CATION CHANNEL PROTEIN (CYCLIC ... P29974/CNG1 MOUSE CGMP-GATED CATION CHANNEL PROTEIN (CYCLIC ... Q62927/CNG1-RAT CGMP-GATED CATION CHANNEL PROTEIN (CYCLIC NU. Q28279|CNG1_CANFA CGMP-GATED CATION CHANNEL PROTEIN (CYCIIC ... Q03043|KGP2 DROME CGMP-DEPENDENT PROTEIN KINASE, ISOZYME 2 F... 
P46148|ETRA_SHEPU ELECTRON TRANSPORT REGULATOR A

GP3_DROME CGMP-DEPENDENT PROTEIN KINASE, ISOZYME 2 F... 40 0.020

P23619|HLYX_ACTPL REGULATORY PROTEIN HLYX

Q14028/CNG4 HUMAN CYCLIC-NUCLEOTIDE-GATED CATION CHANNEL $4 \quad \ldots \quad \ldots \quad 400.027$

Q28181/CNG4_BOVIN 240K PROTEIN OF ROD PHOTORECEPTOR CNG-CHAN... $40 \quad 0.027$

Q03611/YN82_CAEEL HYPOTHETICAL 88.6 KD PROTEIN ZC84.2 IN CHR... 390.035

P12368|KAP2 RAT CAMP-DEPENDENT PROTEIN KINASE TYPE II-ALPHA. $39 \quad 0.046$

P00515|KAP2_BOVIN CAMP-DEPENDENT PROTEIN KINASE TYPE II-ALPH... $38 \quad 0.14$

Q64359/CNGX RAT CYCLIC-NUCLEOTIDE-GATED OLFACTORY CHANNEL OC. $37 \quad 0.18$

P55934/CNG İCTPU CYCLIC-NUCLEOTIDE-GATED CATION CHANNEL $\quad 37 \quad 0.23$

P36165/YK6 $\overline{9}$ YEAST HYPOTHETICAL 102.7 KD PROTEIN IN PRP16-SRP... $37 \quad 0.23$

P12367|KAP2_MOUSE CAMP-DEPENDENT PROTEIN KINASE TYPE II-ALPH . . $37 \quad 370.23$

P54513/YQHO BACSU HYPOTHETICAL 32.9 KD PROTEIN IN GCVT-SPOII... $36 \quad 0.30$

P06170|CRP SALTY CATABOLITE GENE ACTIVATOR (CAMP RECEPTOR PR.. $36 \quad 0.30$

P24290|FX24 RHILV PROBABLE TRANSCRIPTIONAL ACTIVATOR (ORF-240) $36 \quad 0.30$

P29283|NTCA SYNP7 GLOBAL NITROGEN REGULATOR $\quad 36 \quad 0.30$

Q16280/CNG2_HUMAN CYCLIC-NUCLEOTIDE-GATED OLFACTORY CHANNEL ... $36 \quad 0.30$

P03020|CRP ËCOLI CATABOLITE GENE ACTIVATOR (CAMP RECEPTOR PR.. $36 \quad 0.40$

P34578/YNX5_CAEEL HYPOTHETICAL 139.4 KD PROTEIN T20G5.5 IN C... $36 \quad 0.40$

P40308/YM93 YEAST HYPOTHETICAL 73.6 KD PROTEIN IN GLC8-PRE5 $\ldots . \quad 360.52$

P46908|FNR BACSU ANAEROBIC REGULATORY PROTEIN $\quad 36 \quad 0.52$

P43564/YFD 4 YEAST HYPOTHETICAL 119.5 KD PROTEIN IN MOB2-RIM1... 350.68

P33779/NTCA_SYNY3 GLOBAL NITROGEN REGULATOR $\quad 35 \quad 0.90$

Q10898/Y05N MYCTU HYPOTHETICAL 53.5 KD PROTEIN CY251.23 350.90

P12624|MACS_BOVIN MYRISTOYLATED ALANINE-RICH C-KINASE SUBSTR... $34 \quad 1.2$

P29281/CRP_HAEIN CATABOLITE GENE ACTIVATOR (CAMP RECEPTOR PR.. $34 \quad 1.5$

P00516|KGPA BOVIN CGMP-DEPENDENT PROTEIN KINASE, ALPHA ISOZY... $34 \quad 1.5$

P37882/CGB1_MESAU G2/MITOTIC-SPECIFIC CYCLIN BI $33 \quad 2.6$

P14619|KGPB HUMAN CGMP-DEPENDENT PROTEIN KINASE, BETA ISOZYM... $33 \quad 3.5$

P22770|ACH7_CHICK NEURONAI ACETYLCHOLINE RECEPTOR PROTEIN, A... $33 \quad 3.5$

Q16281/CNG3-HUMAN CYCLIC-NUCLEOTIDE-GATED CATION CHANNEL $3 \quad \ldots \quad \ldots 33 \quad 3.5$

P29286/FIXK_BRAJA NITROGEN FIXATION REGULATION PROTEIN FIXK $\quad 32 \quad 4.5$

P32492|MYS4 YEAST MYOSIN-4 ISOFORM

Q08530|BTR BORPE TRANSCRIPTIONAL REGULATORY PROTEIN BTR

P55222/VER PSEAE CYCLIC AMP RECEPTOR-LIKE PROTEIN

P50576|HMD4 BRARE HOMEOBOX PROTEIN DLX-4 $\quad 32 \quad 5.9$

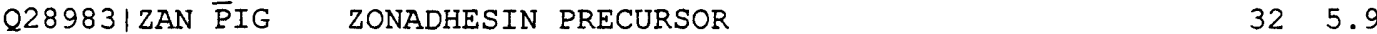

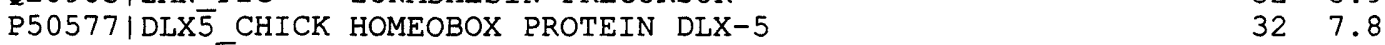

\section{SP|Q02331/YOL4_CAEEL HYPOTHETICAL 152.4 KD PROTEIN ZK370.4 IN CHROMOSOME III Length $=1351$}

Score $=680$ bits $(1736)$, Expect $=0.0$

Identities $=381 / 810(47 \%)$, Positives $=515 / 810(63 \%)$, Gaps $=44 / 810(5 \%)$

Query: 476

Sbjct : 465

Query: 531

Sbjct: 525

Query: 591

Sbjct: 585

Query: 651

Sbjct: 640

Query: 711

$P F A+$

LNSRVLLHHAKAGTI IARQGDQDVSLHEVLWGCLHVYQRMI-----DKAEDVCLFVAQPG 530 $+\mathrm{S}++\mathrm{L} \quad+\mathrm{AG}+\mathrm{I}$ QG $++\mathrm{L}$ VL G L $+Q+2++++$ IGSKIHLESYEAGHVI IEQGAEEEVLMMVLHGNLILAQESLFDEENNEEDESAVIRVTAR 524

Sbjct: 698 APFAAPTVIRT---NELGATDPMSHIKNLHTIAVVPASPDVPLVPFTCELYHALSSNLRV 754

Query: 766 LLLNSDIIRARLGASALDSIQEFRLSGWLAQQEDAHRIVLYQTDASLTPWTVRCLRQADC 825 Sbjct: 755 LRLSSQKVAACLDPSVLEKQADFRLMHWLNVQEDTYPLVIYECDFTQTNWTRRCLRQADA 814 
Query: 826 ILIVGLGDQEPTLGQLEQMLENT---AVRALKQLVLLHREEGAGPTRTVEWLNMRSWCSG 882

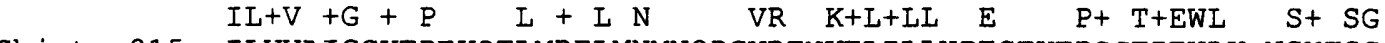
Sbjct: 815 ILVVAIGGKTPEKQTLMRELMNMNQDGVRTNKELILLWPESTKTPSGTIEWLK-NSYFSG 873

Query: 883 HLHLRCPRRL-----FSRRSPAKLH-------ELYEKVFSRRADRHSDFSRLARVL 926 $\begin{array}{lllll} & \text { H H+R P+R+ } & \text { F }+ \text { S A+t } & \text { E YEK } & \text { DR SDFSRLAR+L } \\ \text { Sbjct: } 874 & \text { HHHIRAPKRMLOWNRKFRKMSRAEVMTPTSVENEVIEYYEKNVFWTPDRRSDFSRLARIL } 933\end{array}$

Query: 927 TGNTIALVLGGGGARGCSHIGVLKALEEAGVPVDLVGGTSIGSFIGALYAEERSASRTKQ 986

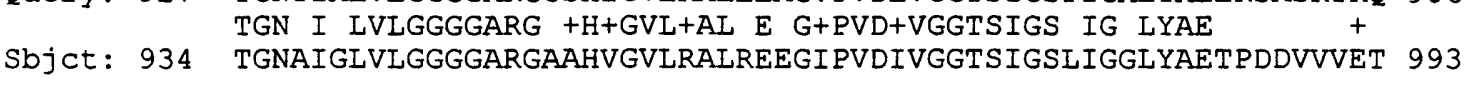

QuerY: 987 RAREWAKSMTSVLEPVLDITYPVTSMFTGSAFNRSIHRVFQDKQIEDLWLPYFNVTTDIT 1046 RA $\mathrm{W}$ M+S+ +LDLTY ++MFTG+ FN SI +F+++ IEDLW+ YF ++TDI+

Sbjct: 994 RAASWFNGMSSLWRKLLDLTYAHSAMFTGAQFNFSIKDLFEERLIEDLWISYFCISTDIS 1053

Query: 1047 ASAMRVHKDGSLWRYVRASMTLSGYLPPLCDPKDGHLLMDGGYINNLPADIARSMGAKTV 1106 $S$ MRVH+ G LW Y RASM+L+GYLPPLCDP+DGHLL+DGGY+NN+PAD+ R++GA+ V

Sbjct: 1054 TSEMRVHRSGPLWAYCRASMSLAGYLPPLCDPQDGHLLLDGGYVNNVPADVMRNLGARCV 1113

Query: 1107 IAIDVGSQDETDLSTYGDSLSGWWLLWKRLNPWADKVKVPDMAEIQSRLAYVSCVRQLEV 1166 IA DVGS +ET+L YGDSLSG $W+L$ KRLNP+ ++ +M EIQSRLAYVSCVRQLEV

Sbjct: 1114 IACDVGSIEETNLYDYGDSLSGMWVLLKRLNPFGTPPRILNMEEIQSRIAYVSCVRQLEV 1173

Query: 1167 VKSSSYCEYLRPPIDCEKTMDFGKFDQIYDVGYQYGKAVFGGWSRGNVIEKMLTDRRST- 1225 $\mathrm{VK}+\mathrm{SYC}$ YLRPPI+ $\mathrm{EKT}+\mathrm{DF} \mathrm{KF}+\mathrm{I}++\mathrm{G}+\mathrm{YG}+\mathrm{N}+\mathrm{L} \mathrm{D}+\mathrm{T}$

Sbjct: 1174 VKKASYCRYLRPPIEPEKTLDFPKFQEIMELGLKYGREACHELIT-NDRAGILGDEKETR 1232

QuerY: 1226 --DLNESRRADVLAFPSSGFTDLAEIVSRI 1253

+ SRR + FTDLA +S+I

Sbjct: 1233 KEKRQQSRREKPDVSRAVSFTDLAAAMSKI 1262

Score $=106$ bits $(262)$, Expect $=2 e-22$

Identities $=87 / 286(30 \%)$, Positives $=134 / 286(46 \%)$, Gaps $=40 / 286(13 \%)$

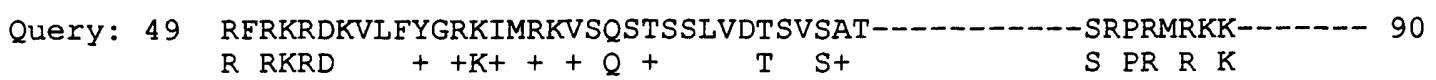

Sbjct: 145 RMRKRD----WAKKLYKSLVQDSPGRTPTDESSDEENANVVMGGGSVPRRRSKHGNSSRR 200

Query: 91 --LKMLNIAKKILRIQKETPTLQRKE-----DPPAVLE-ADLTEGDLANSHLPSEVL 139

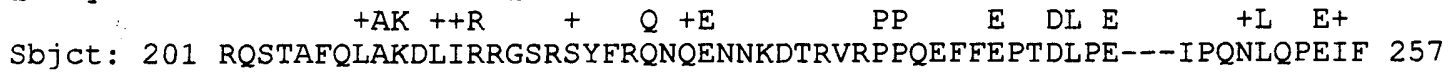

Query: 140 YMLKNVRVLGHFEKPLFLELC-RHMVFQRLGQGDYVFRPGQPDASIYVVQDGLLELCLPG 198 $\mathrm{Y}+\mathrm{L} \mathrm{N}+++\mathrm{L}$ E P + $\mathrm{L} \mathrm{R}++$ GDY + + PG $+\mathrm{D}+\mathrm{IYV}$ DG $\mathrm{L}++$

Sbjct: 258 YILHNLKML---ELPSEWKLDPREIEVRSFQAGDYIVKPGESDDAIYVAIDGELTVHIRH 314

Query: 199 PDGKECVVKEVVPGDSVNSLLSILDVITGHQHPQRTVSARAARDSTVLRLPVEAFSAVFT 258 $+\mathrm{GKE}+\mathrm{VK}+\mathrm{GS}$ SLLS+LD++ $\mathrm{RTV}++\mathrm{AA} \quad \mathrm{V}+\mathrm{P}++\mathrm{F}+$

Sbjct: 315 MEGKEYLVKCIPAGGSFFSLISMLDILMDFPSIFRTVALKAADPCRVAKFPITSFRESYH 374

Query: 259 KYPESLVRVVQIIMVRLQRVTFLALHNYLGLTNELFSHEIQPLRLF 304

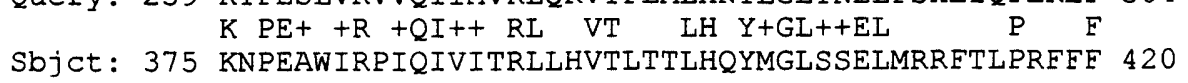

Score $=34.0$ bits $(76)$, Expect $=1.5$

Identities $=28 / 116(24 \%)$, Positives $=51 / 116(43 \%)$, Gaps $=15 / 116(12 \%)$

Query: 597 IDWTAVEAGRALYRQGDRSDCTYIVLNGRLRSVIQRGSGKKELVGEYGRG----DLIGVV 652

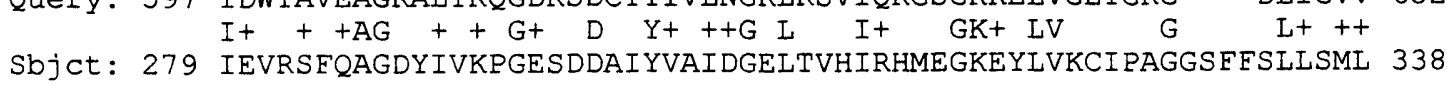

Query: 653 EALTRQP---RATTVHAVRDTELAKLPEGTLGHIKRRYPQ--------VVTRLIHL 697

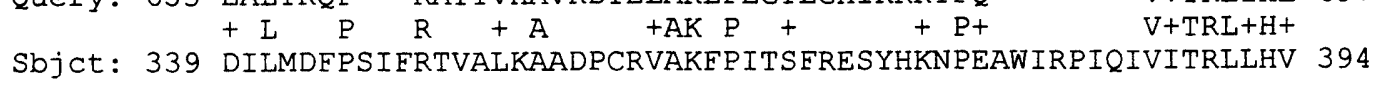


Score $=34.0$ bits $(76)$, Expect $=1.5$

Identities $=24 / 117(20 \%)$, Positives $=52 / 117$ (43\%), Gaps $=6 / 117$ (5\%)

Query: 592 QMDFAIDWTAVEAGRALYRQGDRSDCTYIVLNGRL----RSVIQRGSGKKE--LVGEYGR 645 $\mathrm{Q}+\mathrm{I}+\mathrm{EAG}+\mathrm{QG}+\mathrm{VL}+\mathrm{G} \mathrm{S}+\mathrm{S}+++\mathrm{V}$ Sbjct: 464 QIGSKIHLESYEAGHVIIEQGAEEEVLMMVIHGNIILAQESLFDEENNEEDESAVIRVTA 523

QuerY: 646 GDLIGVVEALTRQPRATTVHAVRDTELAKLPEGTLGHIKRRYPQVVTRLIHLLSQKI 702 $+\mathrm{L}+\mathrm{G}++\mathrm{LT}+\mathrm{P} \mathrm{T}+\mathrm{A} \mathrm{T}+\mathrm{A}+++\mathrm{P}+++\mathrm{H}++++$ Sbjct: 524 RELVGGLQILTNEPSFYTIRAAVQTRVAIMKKKDFSAFLEAHPEIYLPVAHSVLRRI 580

Score $=32.8$ bits $(73)$, Expect $=3.5$

Identities $=28 / 101(27 \%)$, Positives $=48 / 101(46 \%)$, Gaps $=12 / 101(11 \%)$

Query: 171 GDYVERPGQPDASIYVVQDGLLELCLPGPDGKECVVKEVVPGDSVNSLLSILDVITGHQH 230

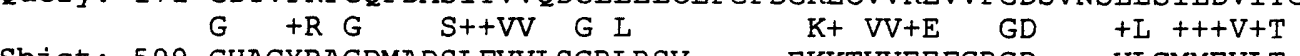

Sbjct: 599 GHACYRAGDMADSLFVVLSGRLRSV-----EKKTVVEEFGRGD----VLGMMEVIT---K 646

QuerY: 231 PQRTVSARAARDSTVLRLPVEAFSAVETKYPESLVRVVQII 271

$\mathrm{R}+\mathrm{ARS}+\mathrm{R}+\mathrm{P}+++\mathrm{YP}+\mathrm{R}+\mathrm{VQ}++$

Sbjct: 647 KPRATTVLAVRFSQLARVPEGLLNFIKMQYPQVGFRLVQLL 687

\section{Sp/Q21534/YVL7_CAEEL HYPOTHETICAL 100.1 KD PROTEIN M110.7 IN CHROMOSOME II Length $=880$}

Score $=503$ bits (1280), Expect $=e^{-141}$

Identities $=279 / 706(39 \%)$, Positives $=400 / 706$ (56\%), Gaps $=55 / 706(7 \%)$

Query: 508 CLHVYQRMIDKAEDVCLFVAQPGELVGQLAVLTGEPLIFTLRAQRDCTFLRISKSDFYEI 567

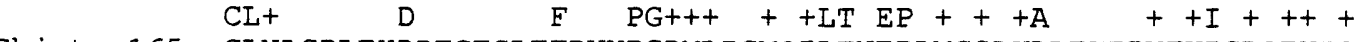

Sbjct: 165 CLNAGDLEHDDTGTSLTEDVYPGDMLPSMQILTNEPAMCSAKALEKTIYFKICRDEYIQE 224

Query: 568 MRAQPSVVLSAAHTVAARMSPFVRQMDFAIDWTAVEAGRALYRQGDRSDCTYIVLNGRLR 627 $+\mathrm{A} P+\mathrm{L} A \quad+S P F R$ D A+ W +E G+AL+RQGD+SD +IV+ GRLR

Sbjct: 225 LFAHPVIYLRLAFHALQFISPFARVFDMAVHWHRIETGQALFRQGDKSDSMHIVMGGRLR 284

Query: 628 SVIQRGSGKKELVGEYGRGDLIGVVEALTRQPRATTVHAVRDTELAKLPEGTLGHIKRRY 687

$+\mathrm{V}+++$ EYGR DIIG + $+++\mathrm{PR}$ TV AVR + + +PE I +K RY

Sbjct: 285 AV-----DSTKIIEEYGRLDLIGITDMAEKRPRRNTVMAVRFSHIVCIPENLISEVKIRY 339

Query: 688 PQVVTRLIHLLSQKILGNLQQLQGPEPAGSGLGVPPHSELTNPASNLATVAILPVCAEVP 747

Sbjct: 340 PQV $+++F$ S VP NSARWSRRDETERSA

SRRVP 362

Query: 748

MVAFTLELQHALQAIGPTLLLNSDIIRARLGASALDSIQEFRLSGWLAQQEDAHRIVLYQ 807 $+\mathrm{ETEL}+\mathrm{L}$ TL $\mathrm{L}+\mathrm{S}++\quad+++\mathrm{L}$ WL $\mathrm{QE} \mathrm{A}+$ +VIYQ

Sbjct: 363 LTEETCELYNQLSKHVKTLRLSSSVVENYEESEVITKKADYGLMHWLNVQEIAYSLVLYQ 422

Query: 808 TDASLTPWTVRCLRQADCILIVGLGDQ---EPTLGQLEQMLENTAVRALKQLVLLHREEG 864 D T WT RCLR AD IL+V LG + E L + VR K+LV L +

Sbjct: 423 CDEHKTNWTRRCLRMADAILMVALGTESKEEQVLAEALLSCNEKGVRQSKELVFLWPIDT 482

Query: 865 AGPTRTVEWLNMRSWCSGHLHLRCPRRLFS---RRSPAKLHELYEKVFSRRADRHSDFSR 921 $\mathrm{P}+\mathrm{T} W+\mathrm{S}+\mathrm{SG}+\mathrm{HLR} \mathrm{P}$ RLES $+\mathrm{K}+\mathrm{E}$ YE SDFSR

Sbjct: 483 PTPSGTAAWIK-ESYYSGYHHLRAPNRLFSFPLKTREKKIVEYYETTVYGEISYQSDFSR 541

Query: 922 LARVITGNTIALVLGGGGARGCSHIGVLKALEEAGVPVDIVGGTSIGSEIGALYA---EE 978

$\begin{array}{lll} & \text { LAR+LTGN I +V GGGGARG +H G L+AL E V +D+VGGTSIG+ G+LYA + } \\ \text { Sbjct: } 542 & \text { LARILTGNAIGIVFGGGGARGAAHAGALRALIEKKVQIDMVGGTSIGALFGSLYATTPDI 601 }\end{array}$

Query: 979 RSASRTKQRAREWAKSMTSVLEPVLDLTYPVTSMFTGSAENRSIHRVEQDKQIEDLWIPY IO38

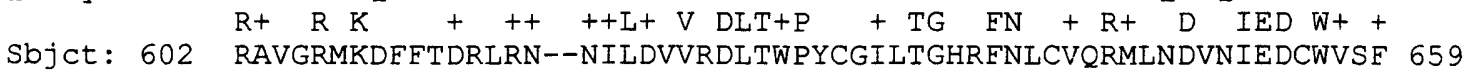

Query: 1039 ENVTTDITASAMRVHKDGSLWRYVRASMTLSGYLPPLCDPKDGHLLMDGGYINNLPADIA 1098

$\mathrm{F}+\mathrm{TTD}+\mathrm{T}+\mathrm{S}+\mathrm{MR}+\mathrm{H}++\mathrm{G}+\mathrm{W} \quad \mathrm{VR}+\mathrm{SM}++\mathrm{GY}+\mathrm{PP}+\mathrm{CDP}+\mathrm{DGHLI}+\mathrm{DG}$ Y+NNLPADI

Sbjct: 660 FCITTDLTSSSMRIHRNGIMWPVVRSSMSIAGYVPPICDPQDGHLLLDGAYVNNLPADIM 719 
Query: 1099 RSMGAKTVIAIDVG-SQDETDLSTYGDSLSGWWLLWKRLNPWADKVKVPDMAEIQSRLAY 1157 $R S+G A \quad V I A I D V G \quad S \quad D \quad T+L \quad Y G \quad S+S G \quad W \quad L+K R \quad P+++++V+M \quad E+Q+R L A Y$ Sbjet: 720 RSLGANVVIAIDVGMSDDNTNLRNYGFSISGTWCLFKRWWPFGEELRVLNMNEVQNRLAY 779

QuerY: 1158 VSCVRQLEVVKSSSYCEYLRPPIDCFKTMDFGKFDQIYDVGYQYGK 1203

$\mathrm{V} C \mathrm{CV}$ Q+E+VK++ YC Y++ PI+ F DF KEDQ +GY K

Sbjct: 780 VCCVNQMEIVKNAQYCYYVKLPIESFGIFDFSKFDQAAQIGYDITK 825

Score $=41.8$ bits $(96)$, Expect $=0.007$

Identities $=39 / 178(21 \%)$, Positives $=71 / 178(38 \%)$, Gaps $=32 / 178(17 \%)$

QuerY: 113 KEPPPAVLEADLTEGDLANSHLPSEVLYMLKNVRVLGHFEKPLFLELC-RHMVFQRLGQG 171 $\mathrm{K}+\mathrm{PP}+\mathrm{E}+\mathrm{E}+\mathrm{SH}+\mathrm{E}+++\mathrm{LK}+\mathrm{L} \mathrm{EP}+\mathrm{L}+++\mathrm{G}$ Sbjct: 15 KQPPRELFEPN--EQEQVPSHIKPEIFFVLKALEGL---ELPTTWQLDPKDVETLSIDTG 69

QuerY: 172 DYVFRPGQPDASIYVVQDGLLELCLPGPDGKECVVKEVVPGDSVNSLLSILDVITGHQHP 231 $\mathrm{V} \mathrm{PG}+\mathrm{I} \mathrm{VV} \mathrm{G} \mathrm{L}+\quad+\mathrm{V}+$

Sbjct: 70 SVVLSPGRANDVIVVVISGELGI-DST 103

Query: 232 QRTVSARAARDSTVLRLPVEAFSAVFTKYPESLVRVVQI IMVRLQRVTFLALHNYLGL 289

$+++\mathrm{A} \quad \mathrm{V}++\mathrm{F} \quad \mathrm{F} \quad \mathrm{P}++\mathrm{R}+\mathrm{Q}++\mathrm{M} R \mathrm{RL}+\mathrm{T}++\mathrm{YLG}+$

Sbjct: 104 NKYIHLKALTSCRVATYHLTSFHTSEIANPQQWIRTIQVVMTRLQQCTLITCNMYLGI 161

\section{SP|Q04958/YMF9_YEAST HYPOTHETICAL $187.1 \mathrm{KD}$ PROTEIN IN OGG1-CNA2 INTERGENIC REGION Length $=1679$}

Score $=452$ bits $(1150)$, Expect $=e-126$

Identities $=278 / 855(32 \%)$, Positives $=440 / 855(50 \%)$, Gaps $=110 / 855(12 \%)$

Query: 459 AAKQELAKLMRIEDPSL-INSRVLLHHAKAGTIIARQGDQDVSLHFVLWGCLHVYQRM-- 515 $\begin{array}{llll}\text { A Kbjet } & 797 \text { AYKEELTPNLDFEDVKKDFAQGIQLKFFKKGTTIVEQNARGKGLFYIISGKVNVTTNSSS } 856\end{array}$ Query: 516 -----IDKAEDVC--_---------LFVAQPGELVGQLAVLTGEPLIFTLRAQRDC 554

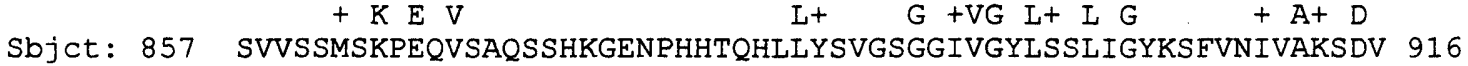
Query: 555 TFLRISKSDFYEIMRAQPSVVLSAAHTVAARMSPFVRQMDFAIDWTAVEAGRALYRQGDR 614 $+\mathrm{S}+++\mathrm{L}++++\mathrm{S}+++\mathrm{DA}++\mathrm{W}+\mathrm{A} \mathrm{L}+\mathrm{QGD}$

Sbjct: 917 YVGFLSSATLERLFDKYFLIYLRISDSLTKLLSSRLLKLDHALEWVHLRASETLFSQGDS 976

Query: 615 SDCTYIVLNGRLRSVIQRGSGKKE---_----LVGEYGRGDLIGVVEALTRQPRATT 663 $\begin{array}{lllll}\text { Sbjct: } 977 \text { ANGIYVVLNGRLRQLQQQSLSNSNTSSEEVETQNIILGELAQGESFGEVEVLTAMNRYST } & 1036\end{array}$

Query: 664 VHAVRDTELAKLPEGTLGHIKRRYPQVVTRLIHLLSQKILGNLQQLQGPFPAGSGLGV-- 721 + AVRD+ELA++P $++\mathrm{P}++\mathrm{R}+\mathrm{L}+++\mathrm{KI}+\mathrm{G}+\quad \mathrm{P} \quad \mathrm{G} \mathrm{I}+$

Sbjct: 1037 IVAVRDSELARIPRTLFELLALEHPSIMIRVSRLVAKKIVGDRTV---PALTGDPLSIKE 1093

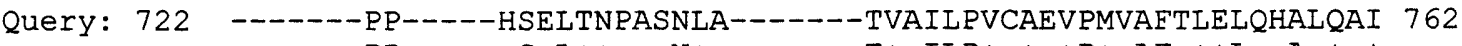

$\begin{array}{cccccc}\text { SP } & \text { S L }++ & \mathrm{N}+ & \mathrm{T}+\text { ILP }+++\mathrm{P}+\mathrm{AF}++\mathrm{A} \text { A }++ & +153\end{array}$

Query: 763 GPTLL-LNSDIIRARLGASALDSIQEFRLSGWLAQQEDAHRIVLYQTDASL-TPWTVRCL 820 $\mathrm{GT}+\mathrm{LN} \quad \mathrm{LG} \mathrm{AD}+++\mathrm{SG}+\mathrm{A}+\mathrm{E}+++\mathrm{V}+\mathrm{Y}+\mathrm{D}++\mathrm{WT} \mathrm{C}+$

Sbjct: 1154 GRTTIGLNQRTTLTHLGRHAFDRLSKLKQSGYFAELEEMYQTVVYISDTPVKSNWTRTCI 1213

Query: 821 RQADCILIVG-LGDQEPTLGQLEQMLENTAVRALKQLVLLHREEGAGPTRTVEWLNMRSW 879 Q DCIL+t +G+ E++L N+ A +L+LLH E $P \quad T$ TL $R$ W Sbjct: 1214 AQGDCILLLADARSPSAEIGEYEKLLLNSKTTARTELILLHPERYVEPGLTHKWLRYRPW 1273

Query: 880 CSGHLHLRCPRRLFSRRSPAKLH-------ELYEKVFSRRADRH 916 $\mathrm{H} \mathrm{H}++\quad+\quad \mathrm{K}+\mathrm{H}+\mathrm{L}+$ FSR +++ Sbjct: 1274 VHSHHHIQFSLTGTTLMNEGKMHVLNNGALALMDKLIQTEFSRKTQQNISKLLPDSTKNT 1333 Query: 917 SDESRLARVLTGNTIALVLGGGGARGCSHIGVLKALE 953 $+D F \quad R L A R+L+G$ I LVLGGGGARG $S H+G V++A+E$

Sbjct: 1334 VENFSSRFMKSKRQYYTPVHRHKNDFLRLARILSGQAIGLVLGGGGARGISHLGVIQAIE 1393 
Query: 954 EAGVPVDLVGGTSIGSFIGALYAEERSASRTKQRAREWAKSMTSVLEPVLDITYPVTSMF 1013 E G+PVD++GGTSIGSF+G LYA++ $\quad \mathrm{R}+++\mathrm{A}++\mathrm{S}++$ DIT+PVTS

Sbjct: 1394 EQGIPVDVIGGTSIGSFVGGLYAKDYDLVPIYGRVKKFAGRISSIWRMLTDLTWPVTSYT 1453

Query: 1014 TGSAFNRSIHRVFQDKQIEDLWLPYFNVTTDITASAMRVHKDGSLWRYVRASMTLSGYLP 1073 $T G \quad F N R I+F D+I E D W+Y++T+I T S$ T $\mathrm{T}$ WRY+RASM+L+G LP

Sbjct: 1454 TGHEFNRGIWKTFGDTRIEDEWIQYYCNSTNITDSVQEIHSFGYAWRYIRASMSLAGLLP 1513

Query 1074 PLCDPKDGHLLMDGGYINNLPADIARSMGAKTVIAIDVGSQDETDLSTYGDSLSGWWLLW 1133 PL $\quad++G+I+D G G Y++N L P \quad R+G+T+A+D V G S$ D+ YGDSL+G+W+++

Sbjct: 1514 PL--EENGSMLLDGGYVDNLPVTEMRARGCQTIFAVDVGSADDRTPMEYGDSLNGFWIIF 1571

Query: 1134 KRLNPWADKVKVPDMAEIQSRLAYVSCVRQLEVVKSSSYCEYLRPPIDCFKTMDFGKFDQ 1193 R NP++ +P+MAEIQ RL YV+ V LE K++ Y+RPPI+ + T+DF KF++

Sbjct: 1572 NRWNPFSSHPNI PNMAEIQVRLGYVASVNALEKAKNTPGVVYVRPPIEEYATLDFSKFEE 1631

Query: 1194 IYDVGYQYGKAVFGG 1208

Sbjct: 1632 IYHVGVDYGRIFLQG 1646

Score $=58.2$ bits $(138)$, Expect $=8 \mathrm{e}-08$

Identities $=25 / 62(40 \%)$, Positives $=40 / 62(64 \%)$

Query: 237 ARAARDSTVLRLPVEAFSAVFTKYPESLVRVVQI IMVRLQRVTFLALHNYLGLTNELFSH 296 ARAA D T + +P ++F + KYP S ++Q +++ +L VTF H+YLGLT E+

Sbjct: 409 ARAATDCTIGIIPPQSFAKLTAKYPRSASHIIQMVLTKLYHVTFQTAHDYLGLTKEIMDI 468

Query: 297 EI 298

Sbjct: $469 \mathrm{EV} 470$

Score $=31.7$ bits $(70)$, Expect $=7.8$

Identities $=32 / 146(218)$, Positives $=61 / 146(408)$, Gaps $=13 / 146(8 \%)$

Query: 140 YMLKNVRVLGHFEKPL---FLELCRHMVFQRLGQGDYVFRPGQPDASIYVVQDGLLE--- 193

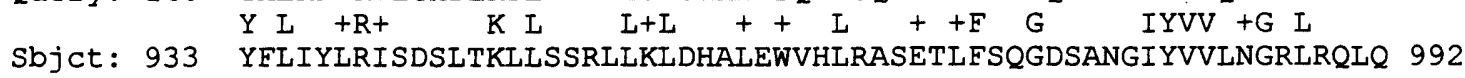

Query: 194 ---LCLPGPDGKECVVKEVVPGD-SVNSLLSILDVITGHQHPQRTVSARAARDSTVLRLP 249

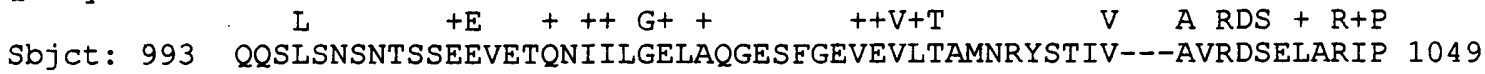

Query: 250 VEAFSAVFTKYPESLVRVVQIIMVRL 275

$\begin{array}{ccc}\text { Sbjct: } & 1050 \text { RTLFELLALEHPSIMIRVSRLVAKKI } 1075\end{array}$

\section{SP|Q50733/YOA3_MYCTU HYPOTHETICAL 62.1 KD PROTEIN CY9C4.03C Length $=583$}

Score $=119$ bits (296), Expect $=2 e-26$

Identities $=69 / 193(35 \%)$, Positives $=109 / 193(55 \%)$, Gaps $=6 / 193(38)$

Query: 919 FSRLARVLTGNTIALVLGGGGARGCSHIGVLKALEEAGVPVDLVGGTSIGSFIGALYAEE 978

F LAR + G ++ LV+ GGGARG +H GV + I EAGV +D GGTS G+ A +A

Sbjct: 295 FGALARRIAGRSLGLVMAGGGARGLAHFGVYQELTEAGVVIDRFGGTSSGAIASAAFALG 354

QuerY: 979 RSASRTKQRAREWAKSMTSVLEPVLDLTYPVTSMFTGSAFNRSIHRVFQDKQIEDLWLPY 1038

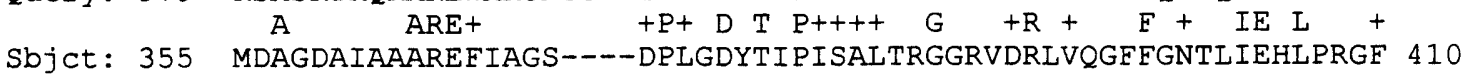

Query: 1039 FNVTTDITASAMRVHKDGSLWRYVRASMTLSGYLPPICDPKDGHLLMDGGYINNLPADIA 1098

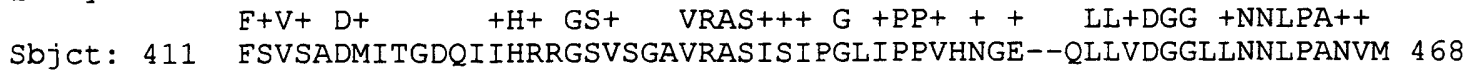


Query: 1099 RSMGAKTVIAIDV 1111

Sbjct: 469 CADTDGEVICVDL 481

Score $=43.8$ bits $(101)$, Expect $=0.002$

Identities $=34 / 120(28 \%)$, Positives $=51 / 120(42 \%)$, Gaps $=11 / 120$ (9\%)

QuerY: 142 LKNVRVLGHFEKPLFLELCRHMVFQRLGQGDYVFRPGQPDASIYVVQDGLLELCLPGPDG 201

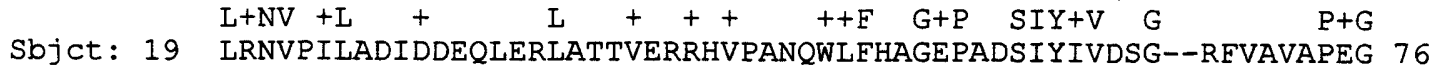
Query: 202 KECVVKEVVPGDSVNSLLSILDVITGHQHPQRTVSARAARDSTVLRLPVEAFSAVFTKYP 261 $\begin{array}{lllll} & \text { V E+ GDS } & \text { L VI G R+ RA RD V R+ E F+ }+ \text { P }\end{array}$ Sbjct: 77 H--VFAEMASGDSIGD----LGVIAG---AARSAGVRALRDGVVWRIAAETFTDMLEATP 127

Score $=36.0$ bits $(81)$, Expect $=0.40$

Identities $=24 / 101(23 \%)$, Positives $=47 / 101(45 \%)$, Gaps $=4 / 101(3 \%)$

Query: 602 VEAGRALYRQGDRSDCTYIVLNGRLRSVIQRGSGKKELVGEYGRGDLIGVVEALTRQPRA 661 $\mathrm{VA}+\mathrm{L}+\mathrm{G}++\mathrm{D}$ YIV $+\mathrm{GR}+\mathrm{V} \mathrm{G}+\mathrm{E} \mathrm{GDIG}++\mathrm{R}+$ Sbjct: 45 VPANQWLFHAGEPADSIYIVDSGRFVAVAPEG----HVFAEMASGDSIGDLGVIAGAARS 100

Query: 662 TTVHAVRDTELAKLPEGTLGHIKRRYPQVVTRLIHLLSQKI 702

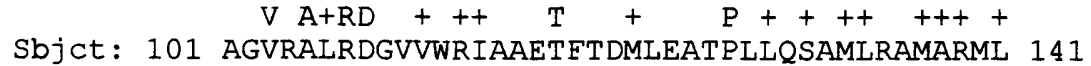
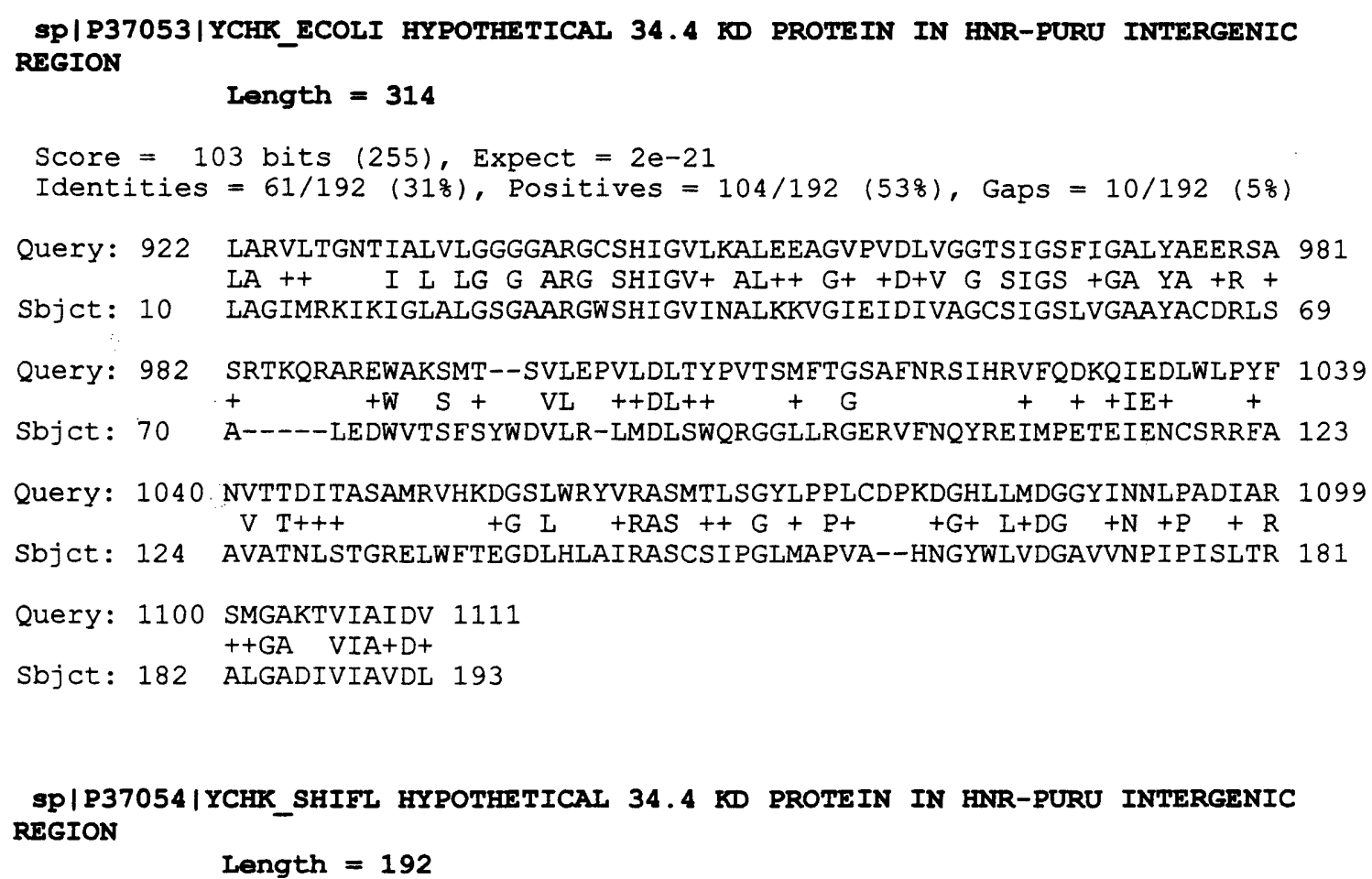
Query: 1041 VTTDITASAMRVHKDGSLWRYVRASMTLSGYLPPLCDPKDGHLLMDGGYINNLPADIARS 1100 V T+++ tG L +RAS ++ G + P+ +G+ L+DG +N +P + R+ Sbjct: 125 VATNLSTGRELWFTEGDLHLAIRASCSIPGLMAPVA--HNGYWLVDGAVVNPIPISLTRA 182

Query: 1101 MGAKTVIAID 1110

+GA VIA+D

Sbjct: 183 IGADIVIAVD 192

\section{SP|P05987|KAPR_DICDI CAMP-DEPENDENT PROTEIN KINASE REGULATORY CHAIN Length $=327$}

Score $=57.4$ bits $(136)$, Expect $=1 e-07$

Identities $=51 / 226(22 \%)$, Positives $=96 / 226(41 \%)$, Gaps $=13 / 226(5 \%)$

Query: 486 KAGTIIARQGDQDVSLHFVLWGCLHVYQRMIDKAEDVCLFVAQPGELVGQLAVLTGEPLI 545 $\mathrm{KAG} I I+Q G D+++G+\mathrm{Y}+++\mathrm{V}+\mathrm{G} G+\mathrm{LA}++\mathrm{G} P$

Sbjct: 88 KAGDIIIKQGDEGDLFYVIDSGICDIYVCQNGGSPTLVMEVFEGGSF-GELALIYGSPRA 146

Query: 546 FTLRAQRDCTFLRISKSDFYEIMRAQPSVVLSAAHTVAARMSPFVRQMDF--------AI 597

$$
\mathrm{T}+\mathrm{A}+\mathrm{D}++++\mathrm{I}+Q \quad++\mathrm{S}+\mathrm{R}+\mathrm{D} \quad \mathrm{A}+
$$

Sbjct: 147 ATVIARTDVRLWALNGATYRRILMDQTIKKRKLYEEFLEKVS-ILRHIDKYERVSLADAL 205

Query: 598 DWTAVEAGRALYRQGDRSDCTYIVLNGRL---RSVIQRGSGKKELVGEYGRGDLIGVVEA 654 $+\quad+\mathrm{G}+\mathrm{RQGD} \mathrm{D} \mathrm{YI}+\mathrm{G}++++\mathrm{VE} \mathrm{D} \mathrm{G}+$

Sbjct: 206 EPVNFQDGEVIVRQGDPGDRFYIIVEGKVVVTQETVPGDHSTSHVVSELHPSDYFGEIAL 265

QuerY: 655 LTRQPRATTVHAVRDTELAKLPEGTLGHIKRRYPQVVTRLIHLLSQ 700

$\mathrm{LT}+\mathrm{PRA} \mathrm{TV}++\mathrm{T}++\mathrm{L}+\mathrm{Q}++\mathrm{R}++\mathrm{Q}$

Sbjct: 266 LTDRPRAATVTSIGYTKCVELDRQRFNRLCGPIDQMLRRNMETYNQ 311

SP|P31319|KAPR_APLCA CAMP-DEPENDENT PROTEIN KINASE REGULATORY CHAIN (N4 SUBUNIT

\section{OFPROTEIN KINASE A)}

Length $=378$

Score $=53.1$ bits $(125)$, Expect $=3 e-06$

Identities $=51 / 202(25 \%)$, Positives $=87 / 202(42 \%)$, Gaps $=18 / 202$ (8\%)

Query: 484 HAKAGTIIARQGDQDVSLHFVLWGCLHVYQRMIDKAEDVCLFVAQPGELVGQLAVLTGEP 543 $\mathrm{H} \quad \mathrm{AG}+\mathrm{I}+\mathrm{QGD}++++\mathrm{G}+\mathrm{VY} \quad+\mathrm{V}+\mathrm{G}$ G+LA+t G P

Sbjct: 154 HRHAGEVIIQQGDEGDNFYVIDQGEVDVY------VNNVHVTSIGEGGSFGELALIYGTP 207

Query: 544 LIFTLRAQRDCTFLRISKSDFYEIMRAQPSVVLSAAHTVAARMSPFVRQMD-------F 595 $\mathrm{T}++\mathrm{A}+\mathrm{D} \mathrm{I}++\mathrm{I}++++\mathrm{S}++\mathrm{D}$

Sbjct: 208 RAATVKAKTDVKLWGIDRDSYRRILMGSTIRKRKIYEDFLSKVS-ILENLDKWERLTVAD 266

Query: 596 AIDWTAVEAGRALYRQGDRSDCTYIVLNGRLRSVIQRGSGKKE--LVGEYGRGDLIGVVE 653 $\mathrm{A}++\mathrm{E}+\mathrm{RQG}+++\mathrm{I}+\mathrm{L} G+\mathrm{V}+\mathrm{QR} S \quad+\mathrm{E}$ VG G $\mathrm{D} G+$

Sbjct: 267 ALEPVQFEDKEEIVRQGEPGEDFFIILEGS-AAVLQRRSENEEPVEVGRLGPSDYFGEIA 325

Query: 654 ALTRQPRATTVHAVRDTELAKL 675

L +PRA TV A + KL

Sbjct: 326 LLIDRPRAATVVARGPLKCVKL 347

\section{SP|P07278|KAPR YEAST CAMP-DEPENDENT PROTEIN KINASE REGULATORY CHAIN Length $=416$}

Score $=51.9$ bits $(122)$, Expect $=6 e-06$

Identities $=47 / 195(24 \%)$, Positives $=84 / 195(42 \%)$, Gaps $=17 / 195(8 \%)$

Query: 488 GTIIARQGDQDVSLHFVLWGCLHVYQRMIDKAEDVCLFVAQPGELVGQLAVLTGEPLIFT 547 G I +QGDQ + V G + Y $\mathrm{D}++\mathrm{PG} \mathrm{G}+\mathrm{LA}++\mathrm{P} \quad \mathrm{T}$ Sbjct: 208 GATIIKQGDQGDYFYVVEKGTVDFY-----VVDNKVNSSGPGSSFGELALMYNSPRAAT 261 
QuerY: 548 LRAQRDCTFLRISKSDFYEIM------RAQPSVVLSAAHTVAARMSPFVRQMDFAIDWT 600 $+\mathrm{A} D \mathrm{DC}++\mathrm{F}+\mathrm{I}+\quad \mathrm{R}+\mathrm{L}++++++\mathrm{A}+\mathrm{D}$

Sbjet: 262 VVATSDCLLWALDRLTERKILLGSSFKKRLMYDDLLKSMPVLKSITTYDRAKLADALDTK 321

QuerY: 601 AVEAGRALYRQGDRSDCTYIVLNGRLRSVIQRGSGKKELVGEYGRGDLIGVVEALTRQPR 660 $+\mathrm{G}+\mathrm{R}+\mathrm{GD}++\mathrm{Y}++\mathrm{G}+\mathrm{V}++\mathrm{G} \mathrm{G}+++\mathrm{D} \mathrm{G} \mathrm{V} \mathrm{L} \mathrm{PR}$ Sbjct: 322 IYQPGETIIREGDQGENFYLIEYGAV-DVSKKGQG---VINKLKDHDYFGEVALLNDLPR 377

Query: 661 ATTVHAVRDTELAKL 675

TV A + T++A L

Sbjct: 378 QATVTATKRTKVATL 392

Score $=32.8$ bits $(73)$, Expect $=3.5$

Identities $=24 / 83(28 \%)$, Positives $=33 / 83(38 \%)$, Gaps $=5 / 83(68)$

QuerY: 601 AVEAGRALYRQGDRSDCTYIVLNGRLRSVIQRGSGKKELVGEYGRGDLIGVVEALTRQPR 660 $+\mathrm{V} G++Q G D+D$ Y $+V$ G $+\quad V$ G G G $++P R$

Sbjct: 204 SVPKGATIIKQGDQGDYFYVVEKGTVDFYVNDNK-----VNSSGPGSSFGELALMYNSPR 258

Query: 661 ATTVHAVRDTELAKLPEGTLGHI 683

$A$ TV A $D$ I $\quad L \quad T \quad I$

Sbjct: 259 AATVVATSDCLLWALDRLTFRKI 281

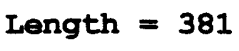

Query: 402 QEEASGGSLAAPARTPTQEPREQPAGACEYSYCEDESATGGCPFGPYQGRQTSSIFEAAK 461 $\mathrm{Q}++\quad++\quad \mathrm{P}+\mathrm{R}+\mathrm{G} \quad \mathrm{YE}++++\mathrm{P}++++\mathrm{A}$

Sbjct: 74 QSDSHDEEVSPTPPNPVVKARRRRGGVSAEVYTEEDAVSYVRKVIPKDYKTMTALAKAIS 133

Query: 462 QEL--AKIMRIEDPSLINSRVLLHHAKAGTIIARQGDQDVSLHFVLWGCLHVYQRMIDKA 519 $++\mathrm{AL} \mathrm{E}++++\mathrm{H} A \mathrm{~A}++Q \mathrm{G}++++\mathrm{V} \mathrm{G}+\mathrm{VY}$

Sbjct: 134 KNVLFAHLDDNERSDIFDAMFPVTHI-AGETVIQQGNEGDNFYVVDQGEVDVY------V 186

Query: 520 EDVCLFVAQPGELVGQLAVLTGEPLIFTLRAQRDCTFLRISKSDF------------Y 565

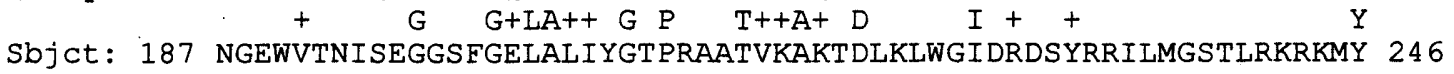

Query: 566 EIMRAQPSVVLSAAH----TVAARMSPFVRQMDFAIDWTAVEAGRALYRQGDRSDCTYIV 621 $\begin{array}{llccc} & \text { E ++ S++ S } & \text { TVA R+ P } & \text { E G + QG+ D YI+ } \\ \text { Sbjct: } 247 \text { EEFLSKVSILESLEKWERLTVADRLEP-- } & \text { P }\end{array}$

Query: 622 LNGRLRSVIQRGSGKKEL--VGEYGRGDLIGVVEALTRQPRATTVHAVRDTELAKL 675 G SV+QR S +E VG G D G + L +PRA TV A + KL Sbjct: 296 TEG-TASVLQRRSPNEEYVEVGRLGPSDYFGEIALLLNRPRAATVVARGPLKCVKL 350

\section{SP|P09456/KAPO RAT CAMP-DEPENDENT PROTEIN KINASE TYPE I-ALPHA REGULATORY CHAIN Length $=381$}

Score $=49.6$ bits $(116)$, Expect $=3 e-05$

Identities $=49 / 199(24 \%)$, Positives $=86 / 199(428)$, Gaps $=18 / 199(9 \%)$

Query: 487 AGTIIARQGDQDVSLHFVLWGCLHVYQRMIDKAEDVCLFVAQPGELVGQLAVLTGEPLIF 546 $A G++Q G D++++G+V Y+V+G \quad G+L A++G P$

Sbjct: 160 AGETVIQQGDEGDNFYVIDQGEMDVYVN-----NEWATSVGEGGSF-GELALIYGTPRAA 213

QuerY: 547 TLRAQRDCTFLRISKSDFYEIMRAQPSVVLSAAHTVAARMSPFVRQMD-------FAID 598 Sbjct: 214 TVKAKTNVKLWGIDRDSYRRILMGSTLRKRKMYEEFLSKVS-IIESLDKWERLTVADALE 272 
QuerY: 547 TLRAQRDCTFLRISKSDFYEIMRAQPSVVLSAAHTVAARMSPFVRQMD--------FAID 598 $\mathrm{T}++\mathrm{A}++\mathrm{I}++\mathrm{I}+\quad+++\mathrm{S}++\mathrm{D}$ A++

Sbjct: 214 TVKAKTNVKLWGIDRDSYRRILMGSTLRKRKMYEEFLSKVS-ILESLDKWERLTVADALE 272

Query: 599 WTAVEAGRAIYRQGDRSDCTYIVLNGRLRSVIQRGSGKKEL--VGEYGRGDLIGVVEALT 656 $\mathrm{E} G++\mathrm{QG}+\mathrm{D}+\mathrm{I}+\mathrm{L} G+\mathrm{V}+\mathrm{QR} S+\mathrm{E}$ VG G D G + I

Sbjct: 273 PVQFEDGQKIVVQGEPGDEFFIILEGS-AAVLQRRSENEEFVEVGRLGPSDYFGEIALIM 331

QuerY: 657 RQPRATTVHAVRDTELAKL 675

+PRA TV A $+\mathrm{KL}$
Sbjct: 332 NRPRAATVVARGPLKCVKL 350

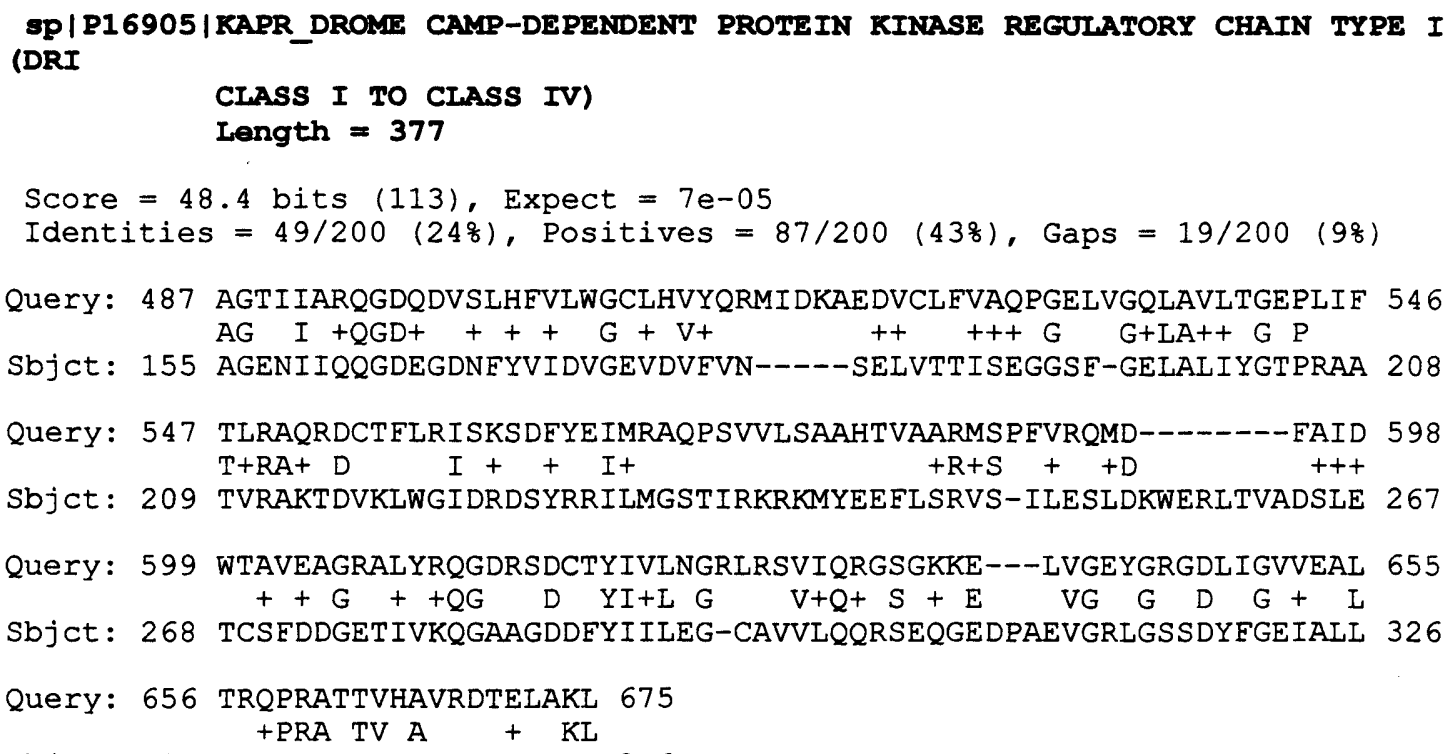

Sbjct: 327 LDRPRAATVVARGPLKCVKL 346

\section{SP|P31322|KAP3_BOVIN CAMP-DEPENDENT PROTEIN KINASE TYPE II-BETA REGULATORY CHAIN \\ Length $=418$}

Score $=47.3$ bits $(110)$, Expect $=2 e-04$

Identities $=71 / 314(22 \%)$, Positives $=117 / 314(36 \%)$, Gaps $=25 / 314(7 \%)$

Query: 375 GDISGLQGG--PRSDFDMAYE-RGRISVSLQEEASGGSLAAPARTPTQEPREQPAGACEY 431

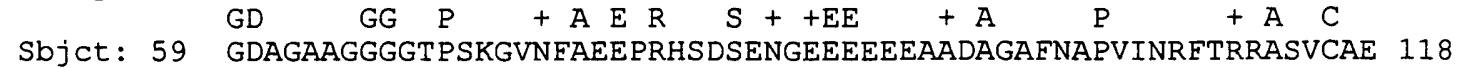

Query: 432 SYCEDESATGGCP--FGPYQGRQTSSIFEAAKQELA-KLMRIEDPSLLNSRVLLHHAKAG 488

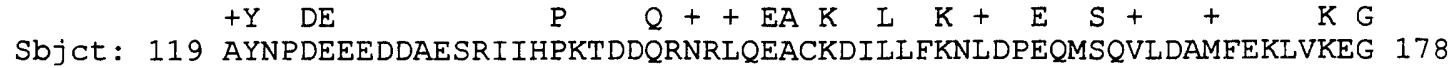

Query: 489 TIIARQGDQDVSLHFVLWGCLHVYQRMIDKAEDVCLFVAQPGELVGQLAVLTGEPLIFTL 548

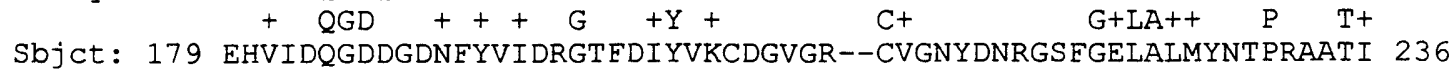

Query: 549 RAQRD-------CTFLRISKSDFYEIMRAQPSVVLSAAHTVAARMSPFVRQMDFAIDWT 600

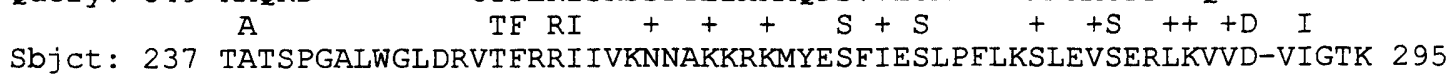
Query: 601 AVEAGRALYRQGDRSDCTYIVLNGRLRSVIQRGSGKKEL-------VGEYGRGDLIGVVE 653

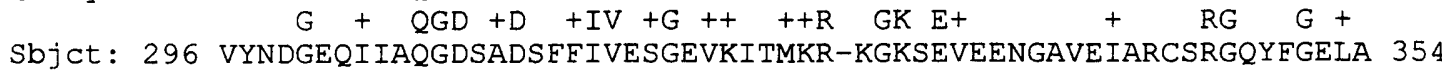
Query: 654 ALTRQPRATTVHAV 667 $+\mathrm{T}+\mathrm{PRA}+\mathrm{HA}+$ Sbjct: 355 LVTNKPRAASAHAI 368 


\section{sp|P07802/KAPO_PIG CAMP-DEPENDENT PROTEIN KINASE TYPE I-ALPHA REGULATORY CHAIN Length $=380$}

Score $=47.3$ bits $(110)$, Expect $=2 e-04$

Identities $=52 / 205$ (25\%), Positives $=89 / 205$ (43\%), Gaps $=30 / 205$ ( $14 \%)$

Query: 487 AGTIIARQGDQDVSLHEVIWGCLHVYQRMIDKAEDVCLFVAQPGELVGQLAVLTGEPLIF 546 $\mathrm{AG}++\mathrm{QGD}++++\mathrm{G}+\mathrm{VY}+\mathrm{V}+\mathrm{G}$ G+LA++ G $\mathrm{P}$

Sbjct: 159 AGETVIQQGDEGDNEYVIDQGEMDVYVN-----NEWATSVGEGGSF-GELALIYGTPRAA 212

Query: 547 TLRAQRDC------ TFLRI-----SKSDFYEIMRAQPSVVLSAAHTVAARMSPFVRQ 592 $\mathrm{T}++\mathrm{A}+++\mathrm{RI} \quad \mathrm{K}$ YE ++ S++ S ++ +

Sbjct: 213 TVKAKTNVKLWGNDRDSYRRILMGSTLRKRKMYEEFLSKVSILESLDKWERLTVADALEP 272

Query: 593 MDEAIDWTAVEAGRALYRQGDRSDCTYIVLNGRLRSVIQRGSGKKEL--VGEYGRGDLIG 650 $+\mathrm{E} \quad \mathrm{E} \mathrm{G}++\mathrm{QG}+\mathrm{D}+\mathrm{I}+\mathrm{L} G+\mathrm{V}+\mathrm{QR} \mathrm{S}+\mathrm{E}$ VG G $\mathrm{D} G$

Sbjct: 273 VQF------EDGQKIVVQGEPGDEFEIILEGS-AAVLQRRSENEEFVEVGRLGPSDYFG 324

Query: 651 VVEALTRQPRATTVHAVRDTELAKL 675

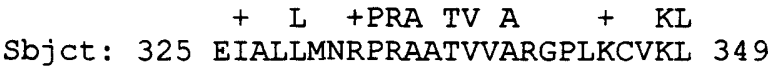

Sp|P12849/KAP1_MOUSE CAMP-DEPENDENT PROTEIN KINASE TYPE I-BETA REGULATORY CHAIN

$$
\text { Length }=381
$$

Score $=46.9$ bits (109), Expect $=2 e-04$

Identities $=60 / 270(22 \%)$, Positives $=109 / 270(40 \%)$, Gaps $=19 / 270(7 \%)$

Query: 417 PTQEPREQPAGACEYSYCEDESATGGCPFGPYQGRQTSSIFEAAKQEL--AKLMRIEDPS 474

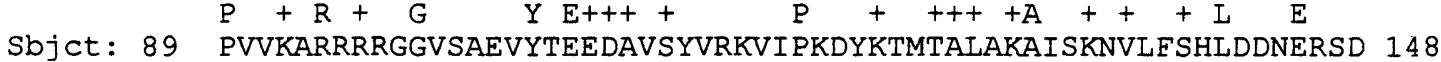

Query: 475 LLNSRVLLHHAKAGTIIARQGDQDVSLHEVLWGCLHVYQRMIDKAEDVCLFVAQPGELVG 534 $++++\mathrm{H} \quad \mathrm{T}+\mathrm{I}+\mathrm{QG}+++++\mathrm{G}+\mathrm{VY}+\mathrm{G} G$

Sbjct: 149 IFDAMEPVTHIGGETVI-QQGNEGDNFYVIDQGEVDVY------VNGEWVTNISEGGSEG 201

Query: 535 QLAVITGEPLIFTLRAQRDCTELRISKSDFYEIM------RAQPSVVISAAHTVAARMS 587 $+\mathrm{LA}+\mathrm{G} P \mathrm{~T}++\mathrm{A}+\mathrm{D} \quad \mathrm{I}++\mathrm{I}+\mathrm{R} \quad \mathrm{LS}++$

Sbjct: 202 ELALIYGTPRAATVKAKTDLKLWGIDRDSYRRILMGSTLRKRKMYEEFLSKVSILESLEK 261

Query: 588 PFVRQMDEAIDWTAVEAGRALYRQGDRSDCTYIVLNGRLRSVIQRGSGKKEL--VGEYGR 645 $+\mathrm{A}++\mathrm{E} G+\mathrm{QG}+\mathrm{D} \mathrm{YI}+\mathrm{G}$ SV+QR $\mathrm{S}+\mathrm{E}$ VG $G$

Sbjct: 262 WERLTVADALEPVQFEDGEKIVVQGEPGDDFYIITEG-TASVLQRRSPNEEYVEVGRLGP 320

QuerY: 646 GDLIGVVEALTRQPRATTVHAVRDTELAKL 675

Sbjct: 321 SDYGGEIALLLNRPRAATVVARGPLKCVKL 350

\section{SP|P47200/ENRA_PSEST TRANSCRIPTIONAI ACTIVATOR PROTEIN FNRA} Length $=244$

Score $=46.1$ bits $(107)$, Expect $=4 \mathrm{e}-04$

Identities $=25 / 108(23 \%)$, Positives $=55 / 108(50 \%)$, Gaps $=1 / 108(0 \%)$

Query: 602 VEAGRALYRQGDRSDCTYIVLNGRLRSVIQRGSGKKELVGEYGRGDLIGVVEALTRQPRA 661

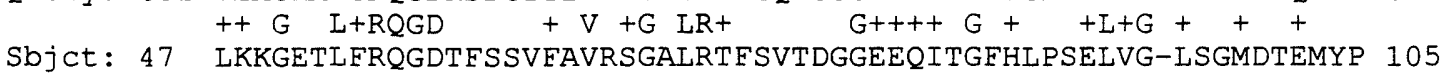

Query: 662 TTVHAVRDTELAKLPEGTLGHIKRRYPQVVTRLIHLLSQKILGNLQQL 709

$\mathrm{T} A+\mathrm{T}+++\mathrm{P} \mathrm{I}+\mathrm{PQ}++\mathrm{L}+++\mathrm{S}+\mathrm{I}+\mathrm{Q}+$

Sbjct: 106 VTAQALETTSVCEIPFERLDELSVILPQLRRQLMRIMSREIRDDQQMM 153 


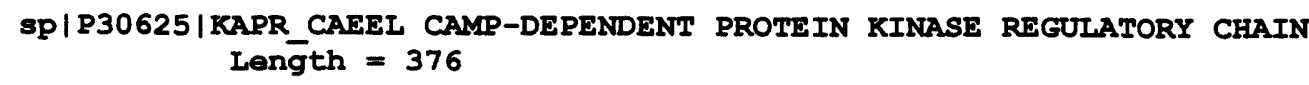

QuerY: 487 AGTIIARQGDQDVSLHFVLWGCLHVYQRMIDKAEDVCLFVAQPGELVGQLAVLTGEPLIF 546 AG I QG $++++G+V Y+\mathrm{L}++\mathrm{G}$ G+LA++ G P

Sbjct: 155 AGETIIEQGEEGDNFYVIDKGTVDVYVN-----HEYVLTINEGGSF-GELALIYGTPRAA 208

Query: 547 TLRAQRDCTFLRISKSDFYEIM------RAQPSVVLSAAHTVAARMSPFVR-QMDFAID 598

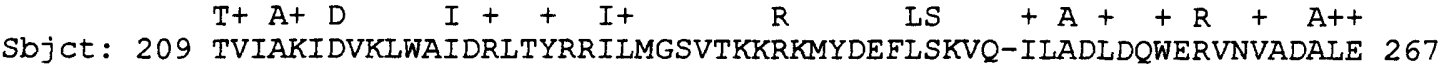

QuerY: 599 WTAVEAGRALYRQGDRSDCTYIVLNGRLRSVIQRGSGKK--ELVGEYGRGDLIGVVEALT 656

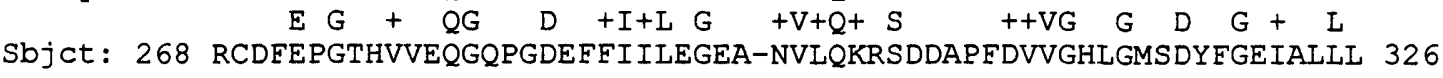
Query: 657 RQPRATTVHAVRDTELAKLPEGTLGHIKRRYPQVVTR 693 $\begin{array}{ll}\text { +PRA TV A } & +\underset{\text { KL }}{\text { Sbjct: }} 327 \text { DRPRAATVVAKTHLKCIKLDRNRFERVMGPVREILKR } 363\end{array}$

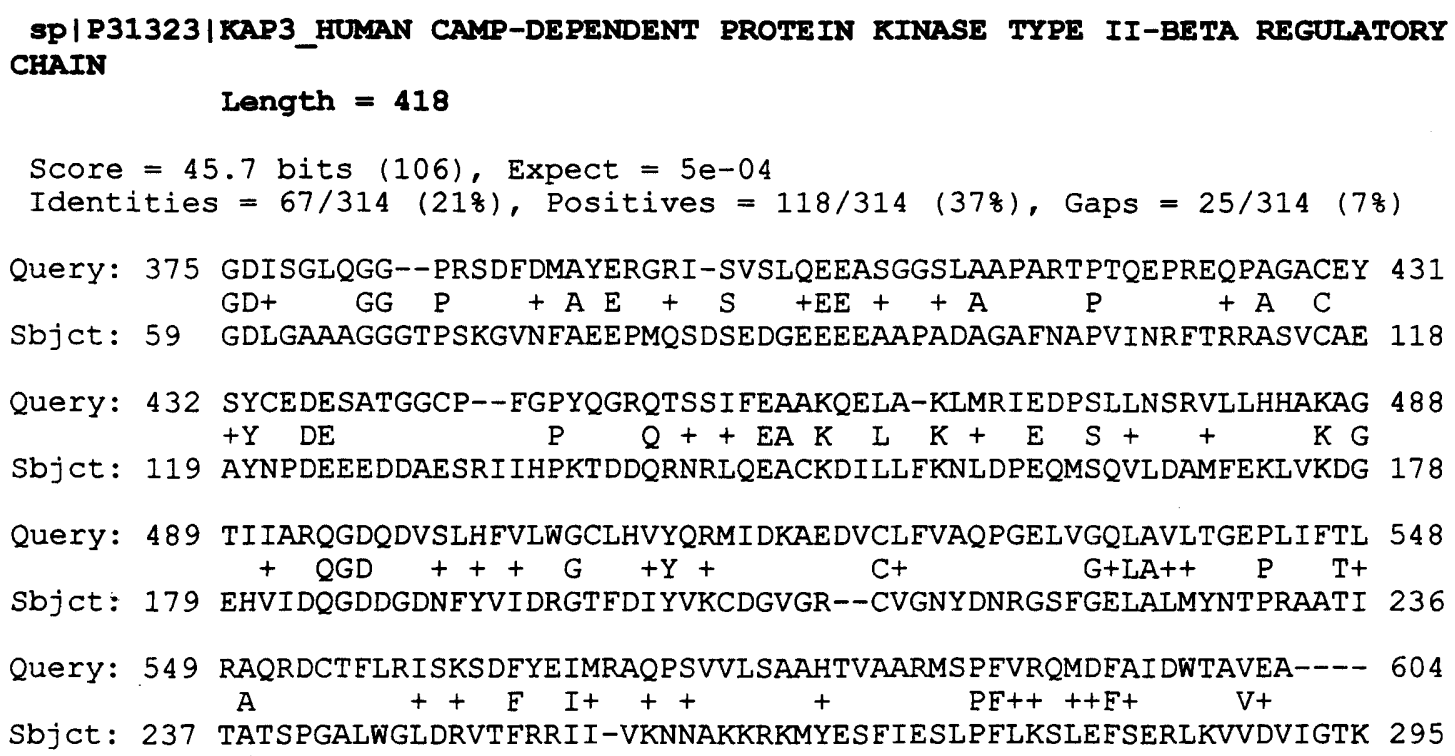


Score $=36.7$ bits $(83)$, Expect $=0.23$

Identities $=27 / 95(28 \%)$, Positives $=48 / 95$ (50\%), Gaps $=8 / 95$ (8\%)

Query: 168 LGQGDYVFRPGQPDASIYVVQDGLLELCLPGPDGKECVVKEVVPGDSVNSLLSILDVITG 227

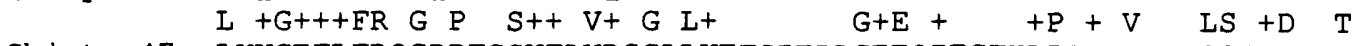
Sbjct: 47 LKKGEFLFRQGDPEGSVFAVRSGALKTFSITDAGEEQITGFHLPSELVG--ISGMDTET- 103

QuerY: 228 HQHPQRTVSARAARDSTVLRLPVEAFSAVFTKYPE 262

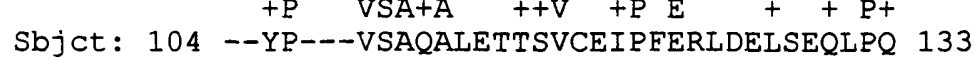

\section{SP|P12369|KAP3 RAT CAMP-DEPENDENT PROTEIN KINASE TYPE II-BETA REGULATORY CHAIN Length $=416$}

Score $=45.3$ bits $(105)$, Expect $=6 e-04$

Identities $=72 / 314(22 \%)$, Positives $=117 / 314(36 \%)$, Gaps $=27 / 314$ (8\%)

Query: 375 GDISGLQGG--PRSDFDMAYERGRISVSLQEE---ASGGSLAAPARTPTQEPREQPAGAC 429

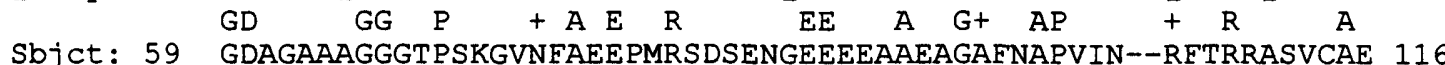

Query: 430 EYSYCEDESATGGCPFGPYQGRQTSSIFEAAKQELA-KLMRIEDPSLLNSRVLLHHAKAG 488 $\mathrm{Y}+\mathrm{E}+\mathrm{E} \quad \mathrm{P} \quad \mathrm{Q}++\mathrm{EAK} \mathrm{L} \mathrm{K}+\mathrm{E} \mathrm{S}++\mathrm{K} \mathrm{G}$

Sbjct: 117 AYNPDEEEDDAESRIIHPKTDDQRNRLQEACKDILLFKNLDPEQMSQVLDAMFEKLVKEG 176

Query: 489 TIIARQGDQDVSLHFVLWGCLHVYQRMIDKAEDVCLFVAQPGELVGQLAVLTGEPLIFTL 548

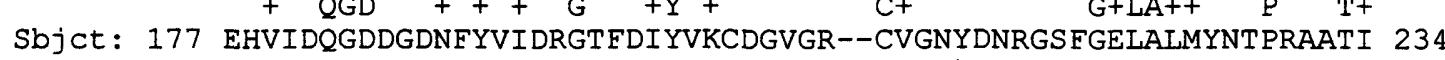

Query: 549 RAQRD-------CTFLRISKSDFYEIMRAQPSVVLSAAHTVAARMSPFVRQMDEAIDWT 600

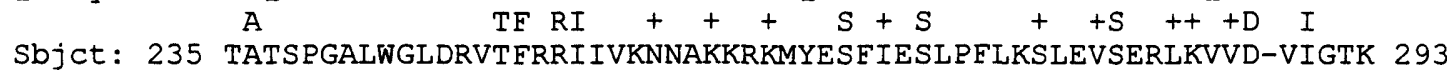
Query: 601 AVEAGRALYRQGDRSDCTYIVLNGRLRSVIQRGSGKKEL-------VGEYGRGDLIGVVE 653 $\begin{array}{cccccc}\text { Sbjct: } & 294 \text { VYNDGEQIIAQGDSADSFFIVESGEVRITMKR-KGKSDIEENGAVEIARCLRGQYFGELA } 352\end{array}$ Query: 654 ALTRQPRATTVHAV 667 $+\mathrm{T}+\mathrm{PRA}+\mathrm{HA}+$ Sbjct: 353 LVTNKPRAASAHAI 366

\section{SP|P37428|FNR_SAITY FUMARATE AND NITRATE REDUCTION REGULATORY PROTEIN Lenḡth $=250$}

Score $=44.5$ bits $(103)$, Expect $=0.001$

Identities $=23 / 104(22 \%)$, Positives $=54 / 104(51 \%)$, Gaps $=1 / 104(08)$

Query: 602 VEAGRALYRQGDRSDCTYIVLNGRLRSVIQRGSGKKELVGEYGRGDLIGVVEALTRQPRA 661 $++\mathrm{G}+\mathrm{L}++\mathrm{GD} \quad \mathrm{Y}++\mathrm{G}++\mathrm{S} \quad \mathrm{G}+++\mathrm{G}+\mathrm{GDL}+\mathrm{G}+\mathrm{A}+$ Sbjct: 52 IQKGQTLFKAGDELKSLYAIRSGTIKSYTITEQGDEQITGFHLAGDLVG-FDAIGSGHHP 110 QuerY: 662 TTVHAVRDTELAKLPEGTLGHIKRRYPQVVTRLIHLISQKILGN 705 $+\mathrm{A}+++++\mathrm{P} \mathrm{TL}++\mathrm{P}++++\mathrm{L}+\mathrm{S}+\mathrm{I} \mathrm{G}+$ Sbjct: 111 SFAQALETSMVCEIPFETLDDLSGKMPNLRQQMMRIMSGEIKGD 154

\section{SP|P03019/FNR_ECOII FUMARATE AND NITRATE REDUCTION REGULATORY PROTEIN Lenḡth $=250$}

Score $=44.5$ bits $(103)$, Expect $=0.001$

Identities $=23 / 104(22 \%)$, Positives $=54 / 104(51 \%)$, Gaps $=1 / 104(0 \%)$

Query: 602 VEAGRALYRQGDRSDCTYIVLNGRLRSVIQRGSGKKELVGEYGRGDLIGVVEALTRQPRA 661 $++\mathrm{G}+\mathrm{L}++\mathrm{GD} \quad \mathrm{Y}++\mathrm{G}++\mathrm{S} \quad \mathrm{G}+++\mathrm{G}+\mathrm{GDL}+\mathrm{G}+\mathrm{A}+$ Sbjct: 52 IQKGQTLFKAGDELKSLYAIRSGTIKSYTITEQGDEQITGFHLAGDLVG-FDAIGSGHHP 110 
Query: 662 TTVHAVRDTELAKLPEGTLGHIKRRYPQVVTRLIHLLSQKILGN 705

$+\mathrm{A}+++++\mathrm{P} \mathrm{TL}++\mathrm{P}++++\mathrm{I}+\mathrm{S}+\mathrm{I} \mathrm{G}+$

Sbjct: 111 SFAQALETSMVCEIPEETLDDLSGKMPNLRQQMMRLMSGEIKGD 154

SP/Q90980/CNG3 CHICK CYCLIC NUCLEOTIDE GATED CHANNEL, ROD PHOTORECEPTOR, ALPHA SUBUNNIT (CNG CHANNNEL 3) (CNG-3) (CNG3) Length $=645$

Score $=44.1$ bits $(102)$, Expect $=0.001$

Identities $=36 / 126(28 \%)$, Positives $=59 / 126(46 \%)$, Gaps $=6 / 126(4 \%)$

Query: 139 LYMLKNVRVLGHFEKPLFLELCRHMVFQRLGQGDYVFRPGQPDASIYVVQDGLLELCLPG 198 I LK VR+ $E \mathrm{~L}+\mathrm{EL}+\mathrm{Q}$ GDY+ R G +Y++++G +L +

Sbjct: 423 LETLKKVRIFADCEAGLLVELVLKLQPQVYSPGDYICRKGDIGREMYIIKEG--KLAVVA 480

Query: 199 PDGKECVVKEVVPGD-SVNSLLSILDVITGHQHPQRTVSARAARDSTVLRIPVEAFSAVF 257

DG $V+V V$ D S +SIL++ +RT + R+ S + L +

Sbjct: 481 DDG---VTQFVVLSDGSYFGEISILNIKGSKAGNRRTANIRSIGYSDLFCLSKDDLMEAL 537

Query: 258 TKYPES 263

$\mathrm{T}+\mathrm{YP}++$

Sbjct: 538 TEYPDA 543

\section{SP|P45199/FNR HAEIN FUMARATE AND NITRATE REDUCTION REGULATORY PROTEIN HOMOLOG Length $=257$}

Score $=44.1$ bits $(102)$, Expect $=0.001$

Identities $=21 / 108(198)$, Positives $=57 / 108(528)$, Gaps $=1 / 108(08)$

Query: 602 VEAGRALYRQGDRSDCTYIVLNGRIRSVIQRGSGKKELVGEYGRGDLIGVVEALTRQPRA 661 $++\mathrm{I}++\mathrm{GD}+\mathrm{Y}++\mathrm{G}++\mathrm{S}$ SG++++ $+\mathrm{GDL}+\mathrm{G}+\mathrm{A}+\mathrm{T}+$

Sbjct: 55 IQKSQVLFKAGDSLNSIYAIRSGTIKSYTISESGEEQITSEHLPGDLVG-FDAITQMQHP 113

Query: 662 TTVHAVRDTELAKLPEGTLGHIKRRYPQVVTRLIHLLSQKILGNLQQL 709

Sbjct : 114 SFAQALETAMVCEIPFDILDDLAGKMPKLRQQILRLMSSEIKSDQEMI 161

Score $=36.0$ bits $(81)$, Expect $=0.40$

Identities $=24 / 98(24 \%)$, Positives $=45 / 98 \quad(45 \%)$, Gaps $=8 / 98 \quad(8 \%)$

Query: 174 VERPGQPDASIYVVQDGLLELCLPGPDGKECVVKEVVPGDSVNSLISILDVITGHQHPQR 233 $+\mathrm{F}+\mathrm{G} \quad \mathrm{SIY}++\mathrm{G}++\quad \mathrm{G}+\mathrm{E}+\quad+$ PGD V $\quad$ D IT QHP

Sbjct: 61 LFKAGDSLNSIYAIRSGTIKSYTISESGEEQITSFHLPGDLVG-----FDAITQMQHPS- 114

Query: 234 TVSARAARDSTVLRLPVEAFSAVFTKYPESLVRVVQII 271

$$
\mathrm{A}+\mathrm{A}+\mathrm{V}+\mathrm{P}++\mathrm{K} \mathrm{P}+++++++
$$

Sbjct: 115 --FAQALETAMVCEIPEDILDDLAGKMPKLRQQILRLM 150

SP/Q50734/YOA4_MYCTU HYPOTHETICAL ABC TRANSPORTER ATP-BINDING PROTEIN CY9C4.04C

$$
\text { Length }=330
$$

Score $=43.8$ bits $(101)$, Expect $=0.002$

Identities $=24 / 68(35 \%)$, Positives $=31 / 68 \quad(45 \%)$

Query: 602 VEAGRALYRQGDRSDCTYIVLNGRLRSVIQRGSGKKELVGEYGRGDLIGVVEALTRQPRA 661 $V+A G \quad L+Q \quad D \quad Y+V \quad G \quad V+G+E L V \quad G D G+I \quad P R+$

Sbjct: 231 VKAGEVLFEQSTMGDLIYVVSEGEFEIVRELADGGEELVKTAAPGDYFGEIGVLFHLPRS 290

Query: 662 TTVHAVRD 669

TV A D

Sbjct: 291 ATVRARSD 298 
Score $=37.9$ bits $(86)$, Expect $=0.10$

Identities $=23 / 83(27 \%)$, Positives $=40 / 83(47 \%)$, Gaps $=1 / 83(1 \%)$

QuerY: 484 HAKAGTIIARQGDQDVSLHFVLWGCLHVYQRMIDKAEDVCLFVAQPGELVGQLAVLTGEP 543 $\mathrm{H} \mathrm{KAG}++\mathrm{Q}++\mathrm{V} \mathrm{G}+++\mathrm{D} \mathrm{E}+++\mathrm{A} \mathrm{PG}+\mathrm{G}++\mathrm{VI} \quad \mathrm{P}$

Sbjct: 230 HVKAGEVLFEQSTMGDLIYVVSEGEFEIVRELADGGEEL-VKTAAPGDYFGEIGVLFHLP 288

QuerY: 544 LIFTLRAQRDCTFLRISKSDFYE 566

Sbjct: 289 RSATVRARSDATAVGYTAQAFRE 311

Score $=37.1$ bits $(84)$, Expect $=0.18$

Identities $=57 / 234(24 \%)$, Positives $=90 / 234(38 \%)$, Gaps $=39 / 234$ (16\%)

Query: 52 KRDKV--LFYGRKIMRKVSQSTSSLVDTSVSATSRPRMRKKLKMLNIAKKILRIOKETP- 108

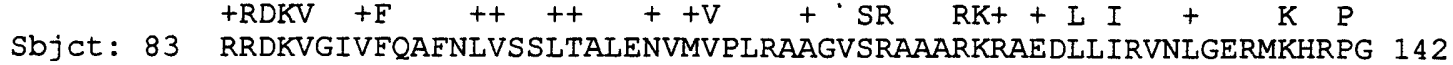

Query: 109 ----TLQRKEPPPAV-LEADLTEGDLANSHLP----SEVLYMLKN-------VRVLGHF 151 QR $\mathrm{A}+\mathrm{L}+\mathrm{L} \quad \mathrm{D}+\mathrm{HL} \quad \mathrm{EVL}++++\quad \mathrm{V} \mathrm{V}$ H

Sbjct: 143 DMSGGQQQRVAVARAIALDPQLILADEPTAHLDFIQVEEVLRLIRSLAQGDRVVVVATHD 202

QuerY: 152 EKPL---DLELCRHMVFQR------LGQGDYVFRPGQPDASIYVVQDGLLELCLPGP 199 $+\mathrm{L} \quad \mathrm{LEL} \quad \mathrm{V}+\mathrm{G}++\mathrm{F} \quad$ IYVV +G E+

Sbjct: 203 SRMLPLADRVLELMPAQVSPNQPPETVHVKAGEVLFEQSTMGDLIYVVSEGEFEIVRELA 262

Query: 200 DGKECVVKEVVPGDSVNSLLSILDVITGHQHPQRTVSARAARDSTVLRLPVEAF 253

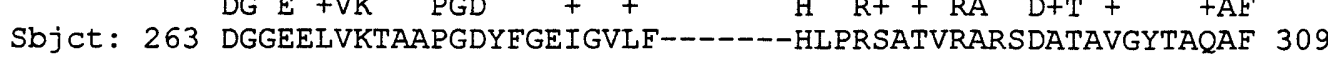

\section{SP|Q03041/CNG2 BOVIN CYCLIC-NUCLEOTIDE-GATED OLFACTORY CHANNEL (CYCLIC- NUCIĒOTIDE-GATED CATION CHANNEL 2) (CNG CHANNEL 2) (CNG-2) (CNG2) Length $=663$}

Score $=42.6$ bits (98), Expect $=0.004$

Identities $=38 / 147(25 \%)$, Positives $=65 / 147(43 \%)$, Gaps $=13 / 147(8 \%)$

Query: 117 PAVLEADLTEGDLANSHLPSEVLYMLKNVRVLGHFEKPLFLELCRHMVFQRLGQGDYVFR 176 PA I A++ $\mathrm{N} \mathrm{HL}+$ LK VR+ E L +EL + Q GDY+ Sbjct: 433 PAKLRAEIA----INVHLST-----IKKVRIFQDCEAGLLVELVLKLRPQVFSPGDYICR 483

Query: 177 PGQPDASIYVVQDGLLELCLPGPDGKECVVKEVVPGDSVNSLLSILDVITGHQHPQRTVS 236 G $+\mathrm{Y}++++\mathrm{G}+\mathrm{L}+\mathrm{DG}++\mathrm{S}+\mathrm{SIL++}+\mathrm{RT}+$ Sbjct: 484 KGDIGKEMYIIKEG--KLAVVADDG--VTQYALLSAGSCFGEISILNIKGSKMGNRRTAN 539

QuerY: 237 ARAARDSTVLRLPVEAFSAVETKYPES 263

Sbjct: 540 IRSLGYSDLFCLSKDDLMEAVTEYPDA 566

SP|Q00195|CNG2_RAT CYCLIC-NUCLEOTIDE-GATED OLFACTORY CHANNEL (CYCLICNUCLEOTIDE-

GATED CATION ChANNEL 2) (CNG CHANNEL 2) (CNG2) (CNG-2) (OCNC1) Length $=664$

Score $=42.6$ bits $(98)$, Expect $=0.004$

Identities $=38 / 147$ (25\%), Positives $=65 / 147$ (43\%), Gaps $=13 / 147$ (8\%)

Query: 117 PAVLEADLTEGDLANSHLPSEVLYMLKNVRVLGHFEKPLFLELCRHMVFQRLGQGDYVFR 176 PA L A + $\quad \mathrm{N} \mathrm{HL}+$ LK VR+ E L +EL $+\mathrm{Q}$ GDY $\mathrm{R}$

Sbjct: 435 PAKLRAEIA----INVHLST-----LKKVRIFQDCEAGLLVELVLKLRPQVFSPGDYICR 485

Query: 177 PGQPDASIYVVQDGLLELCLPGPDGKECVVKEVVPGDSVNSLLSILDVITGHQHPQRTVS 236 $\mathrm{G}+\mathrm{Y}++++\mathrm{G}+\mathrm{L}+\mathrm{DG}++\mathrm{S}+\mathrm{SIL}++\quad+\mathrm{RT}+$ Sbjct: 486 KGDIGKEMYIIKEG--KLAVVADDG--VTQYALLSAGSCFGEISILNIKGSKMGNRRTAN 541 
Query: 237 ARAARDSTVLRLPVEAFSAVFTKYPES 263

$\mathrm{R}+\mathrm{S}+\mathrm{L}+\mathrm{T}+\mathrm{YP}++$

Sbjct: 542 IRSLGYSDLFCLSKDDLMEAVTEYPDA 568

\section{SP|Q28718/CNG2_RABIT CYCLIC-NUCLEOTIDE-GATED OLFACTORY CHANNEL (CYCLINUCLEOTIDE- \\ GATED CATION CHANNEL 2) (CNG ChANNEL 2) (CNG-2) (CNG2) (AORTA CNG CHANNEL) (RACNG) \\ Length $=664$}

Score $=42.6$ bits $(98)$, Expect $=0.004$

Identities $=38 / 147(25 \%)$, Positives $=65 / 147$ (43\%), Gaps $=13 / 147$ (88)

Query: 117 PAVLEADLTEGDLANSHLPSEVLYMLKNVRVLGHFEKPLFLELCRHMVFQRLGQGDYVFR 176 PA L A++ $\mathrm{N} \mathrm{HL}+$ LK VR+ E L +EL $+Q$ GDY $+\mathrm{R}$

Sbjct : 433 PAKLRAEIA----INVHLST-----IKKVRIFQDCEAGLLVELVLKLRPQVFSPGDYICR 483

Query: 177 PGQPDASIYVVQDGLLELCLPGPDGKECVVKEVVPGDSVNSLLSILDVITGHQHPQRTVS 236 G $+\mathrm{Y}++++\mathrm{G}+\mathrm{L}+\mathrm{DG}++\mathrm{S}+\mathrm{SIL}++\quad+\mathrm{RT}+$

Sbjct: 484 KGDIGKEMYIIKEG--KLAVVADDG--VTQYALLSAGSCFGEISILNIKGSKMGNRRTAN 539

Query: 237 ARAARDSTVLRLPVEAFSAVFTKYPES 263

$\mathrm{R}+\mathrm{S}+\mathrm{L}+\mathrm{T}+\mathrm{YP}++$

Sbjct: 540 IRSLGYSDLFCLSKDDLMEAVTEYPDA 566

\section{SP|P31320|KAPR_BLAEM CAMP-DEPENDENT PROTEIN KINASE REGULATORY CHAIN Length $=403$}

Score $=42.2$ bits (97), Expect $=0.005$

Identities $=44 / 196(228)$, Positives $=79 / 196(39 \%)$, Gaps $=8 / 196(4 \%)$

Query: 487 AGTIIARQGDQDVSLHFVLWGCLHVYQRMIDKAEDVCLFVAQPGELVGQLAVLTGEPLIF 546 $A G++R Q G \quad+V$ G L V+ $D V+\quad G \quad G+L A++\quad P$

Sbjct: 179 AGEVVIRQGGVGDYFYVVETGALDVFVNRNGNG-DVKVTDYSAGGSFGELALMYNAPRAA 237

QuerY: 547 TLRAQRDCTFLRISKSDFYEIMRAQPS-----VVLSAAHTVAARMSPFVR-QMDFAIDW 599 $\mathrm{T}+\mathrm{A}+++\mathrm{F}+\mathrm{S}+++\mathrm{P}+\mathrm{R}++\mathrm{A}++$

Sbjct: 238 TVVATAESVLWALDRVTERRILMDHTSRKRRMYEAFLEEVPLLSSLEPYERHKIADALES 297

Query: 600 TAVEAGRALYRQGDRSDCTYIVLNGRLRSVIQRGSGKKELVGEYGRGDLIGVVEALTRQP 659

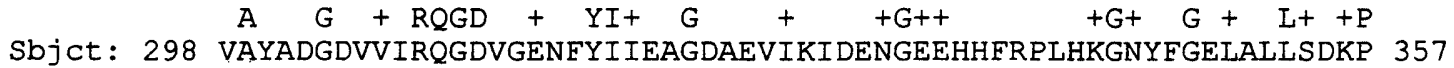

Query: 660 RATTVHAVRDTELAKL 675

$R \quad T+A+A K L$

Sbjct: 358 RVATIRAKGKLKCAKL 373

Score $=41.0$ bits $(94)$, Expect $=0.012$

Identities $=32 / 105(30 \%)$, Positives $=42 / 105(39 \%)$, Gaps $=7 / 105$ (6\%)

Query: 602 VEAGRALYRQGDRSDCTYIVLNGRLRSVIQRGSGKKELVGEYGRGDLIGVVEALTRQPRA 661 $V A G+R Q G \quad D Y+V G L+R \quad V+Y G G++$ PRA Sbjct: 177 VAAGEVVIRQGGVGDYFYVVETGALDVFVNRNGNGDVKVTDYSAGGSFGELALMYNAPRA 236

QuerY: 662 TTVHAVRDTELAKLPEGTLGHI-------KRRYPQVVTRLIHLLS 699

$$
\text { TV A ++ L L T I KRR + + LLS }
$$

Sbjct: 237 ATVVATAESVLWALDRVTFRRILMDHTSRKRRMYEAFLEEVPLLS 281

Score $=32.5$ bits $(72)$, Expect $=4.5$

Identities $=19 / 81(23 \%)$, Positives $=39 / 81 \quad\left(47 \frac{\%}{8}\right)$, Gaps $=1 / 81\left(1 \frac{\circ}{8}\right)$

Query: 488 GTIIARQGDQDVSLHFVLWGCLHVYQRMIDKAEDVCLFVAQPGELVGQLAVLTGEPLIFT 547 $\mathrm{G}++\mathrm{RQGD}+++\mathrm{G} \quad \mathrm{V}+++\mathrm{E}+\quad \mathrm{G} \mathrm{G}+\mathrm{LA}+\mathrm{L}++\mathrm{P}+\mathrm{T}$

Sbjct: 303 GDVVIRQGDVGENFYIIEAGDAEVI-KIDENGEEHHFRPLHKGNYFGELALLSDKPRVAT 361 
Query: 548 LRAQRDCTFLRISKSDFYEIM 568

$+R A+\quad++K \quad F++$

Sbjct: 362 IRAKGKLKCAKLGKKAFTRLL 382

\section{SP|Q24278/CNG_DROME CYCLIC-NUCLEOTIDE-GATED CATION CHANNEL (CNG CHANNEL) Lenḡth $=665$}

\section{Score $=42.2$ bits (97), Expect $=0.005$}

Identities $=34 / 128(268)$, Positives $=54 / 128(418)$, Gaps $=4 / 128(38)$

Query: 142 LKNVRVLGHFEKPLFLELCRHMVFQRLGQGDYVFRPGQPDASIYVVQDGLLELCLPGPDG 201 $\mathrm{LK} V R+\quad E \quad \mathrm{~L} I+Q \quad \mathrm{I} D Y+R G+\mathrm{Y}+\mathrm{V}+\mathrm{G}+\mathrm{L}+\mathrm{G}$ DG

Sbjct: 424 LKQVRIFHDTEPGLLEALVLKLKLQVESPGDYICRKGDVGKEMYIVKRG--KLSVVGDDG 481

Query: 202 KECVVKEVVPGDSVNSLLSILDVITGHQHPQRTVSARAARDSTVLRLPVEAFSAVETKYP 261 $\begin{array}{llllll}\text { Sbjct: } & 482 & \text {--ITVLATLGAGSVFGEVSVLEIAGNRTGNRRTANVRSLGYSDLFCLAKRDLWETISDYP } & +\mathrm{V} 39\end{array}$

Query: 262 ESLVRVVQ 269

Sbjct: 540 EARSTLTQ 547

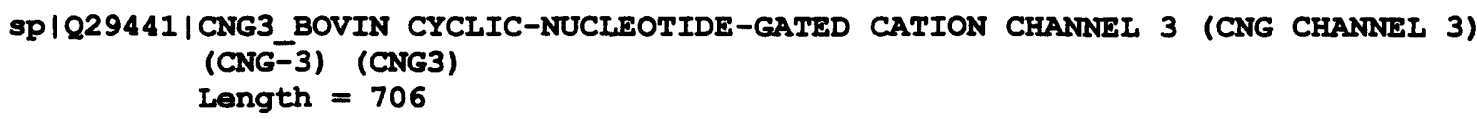

Query: 142 LKNVRVLGHFEKPLFLELCRHMVFQRLGQGDYVFRPGQPDASIYVVQDGLLELCLPGPDG 201 $\mathrm{L}+\mathrm{VR}+\mathrm{E} \mathrm{L}+\mathrm{EL}+\mathrm{GDY}+\mathrm{G}+\mathrm{Y}++++\mathrm{G}+\mathrm{L}+\mathrm{DG}$ Sbjct: 496 LRKVRIFQDCEAGLLVELVLKLRPAVFSPGDYICKKGDIGREMYIIKEG--KLAVVAEDG 553

QuerY: 202 KECVVKEVVPGD-SVNSLISILDVITGHQHPQRTVSARAARDSTVLRLPVEAFSAVFTKY 260

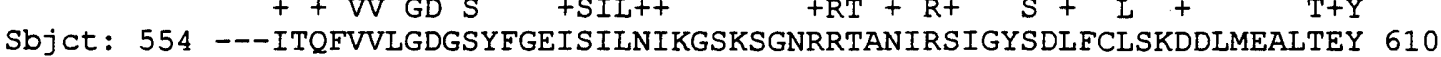

Query: 261 PES 263

Sbjct: 611 PEA 613

\section{SP|P13861/KAP2 HUMAN CAMP-DEPENDENT PROTEIN KINASE TYPE II-ALPHA REGULATORY CHAIN Length $=404$}

Score $=41.4$ bits $(95)$, Expect $=0.009$

Identities $=69 / 317(21 \%)$, Positives $=125 / 317(38 \%)$, Gaps $=28 / 317$ (8\%)

Query: 417 PTQEPREQPAGACEYSYCEDESATGGCPFGPYQGRQTSSIFEAAKQELA-KLMRIEDPSL 475

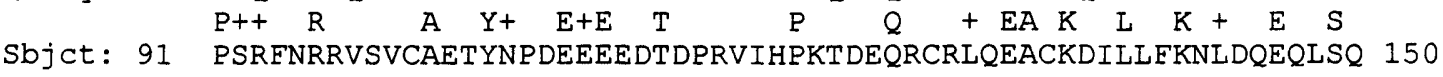

Query: 476 LNSRVLLHHAKAGTIIARQGDQDVSLHFVLWGCLHVYQRMIDKAEDVCLFVAQPGELVGQ 535

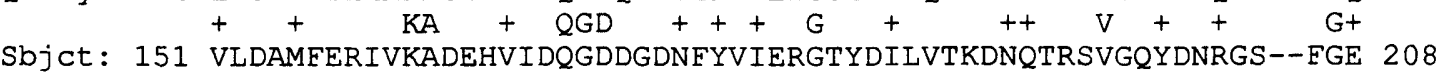

Query: 536 LAVLTGEPLIFTLRAQRD--_---CTFLRISKSDFYEIMRAQPSVVLSAAHTVAARMS 587 $\mathrm{LA}+\mathrm{P} \mathrm{T}+\mathrm{A}+\mathrm{TFRI}+++\mathrm{S}+\mathrm{S}++\mathrm{S}$

Sbjct: 209 LALMYNTPRAATIVATSEGSLWGLDRVTFRRIIVKNNAKKRKMFESFIESVPLLKSLEVS 268

Query: 588 PFVRQMDFAIDWTAVEAGRALYRQGDRSDCTYIVLNG------RLRSVIQRGSGKKEL-V 640

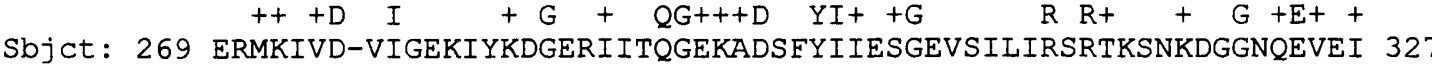


Query: 641 GEYGRGDLIGVVEALTRQPRATTVHAVRDTELAKLP----EGTLGH----IKRRYPQVVT 692
+G G + +T +PRA + +AV D + + E LG +KR Sbjct: 328 ARCHKGQYFGELALVTNKPRAASAYAVGDVKCLVMDVQAFERLLGPCMDIMKRNISHYEE 387

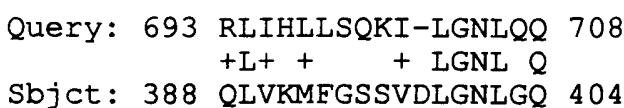

SP|Q90805/CNG1_CHICK CYCLIC NUCLEOTIDE GATED CHANNEL, CONE PHOTORECEPTOR, ALPHA

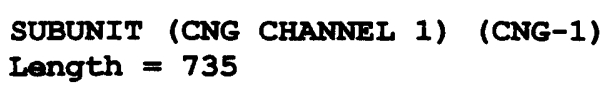

Query: 142 LKNVRVLGHFEKPLFLELCRHMVFQRLGQGDYVFRPGQPDASIYVVQDGLLELCIPGPDG 201 $\mathrm{LK} \mathrm{VR}+\mathrm{E} \mathrm{L}+\mathrm{EL}+\mathrm{GDY}+\mathrm{G}+\mathrm{G}+++\mathrm{G}+\mathrm{L}+\mathrm{DG}$ Sbjct: 519 LKKVRIFODCEAGLLIELVLKLKPTVESPGDYICKKGDIGREMYIIKEG--KLAVVADDG 576 Query: 202 KECVVKEVVPGD-SVNSLLSILDVITGHQHPQRTVSARAARDSTVLRLPVEAFSAVFTKY 260

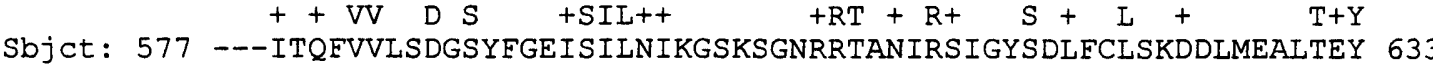

Query: 261 PES 263 $\mathrm{PE}+$

Sbjct: 634 PEA 636

SP/Q62398/CNG2 MOUSE CYCLIC-NUCLEOTIDE-GATED OLFACTORY CHANNEL (CYCLICNUCLẼTIDE-GATED CATION CHANNEL 2) (CNG CHANNEL 2) (CNG-2) (CNG2) Length $=664$

Score $=41.4$ bits (95), Expect $=0.009$

Identities $=38 / 147(25 \%)$, Positives $=65 / 147(43 \%)$, Gaps $=13 / 147(8 \%)$

Query: 117 PAVLEADLTEGDLANSHLPSEVLYMLKNVRVLGHFEKPLFLELCRHMVFQRLGQGDYVFR 176 PA I A++ $\quad \mathrm{N} \mathrm{HL}+$ LK VR+ $\mathrm{E} \mathrm{L} \mathrm{+EL}+Q$ GDY+ $R$ Sbjct: 435 PAKLRAEIA----INVHLST-----LKKVRIFQDCEAGLLVELVLKLRPQVFSPGDYICR 485

Query: 177 PGQPDASIYVVQDGLLELCLPGPDGKECVVKEVVPGDSVNSLLSILDVITGHQHPQRTVS 236 G $+\mathrm{Y}++++\mathrm{G}+\mathrm{L}+\mathrm{DG}++\mathrm{S}+\mathrm{SIL}++$ +RT +

Sbjct: 486 KGDIGKEMYIIKEG--KLAVVADDG--VTQYALLSAGSCFGEISILNIKGSKMGNRRTGT 541

Query: 237 ARAARDSTVLRLPVEAFSAVFTKYPES 263

$\mathrm{R}+\mathrm{S}+\mathrm{L}+\mathrm{T}+\mathrm{YP}++$

Sbjct: 542 IRSLGYSDLFCLSKDDLMEAVTEYPDA 568

SP|P29973/CNG1_hUMAN CGMP-GATED CATION CHANNEL PROTEIN (CYCLIC NUCLFOTIDE GATED

CHANNEL, PHOTORECEPTOR) (CYCLIC-NUCLEOTIDE-GATED CATION CHANNEL 1) (CNG CHANNEL 1) (CNG-1) (CNG1)

Length $=686$

Score $=41.4$ bits (95), Expect $=0.009$

Identities $=33 / 123(26 \%)$, Positives $=58 / 123(46 \%)$, Gaps $=6 / 123(4 \%)$

Query: 142 LKNVRVLGHFEKPLFLELCRHMVFQRLGQGDYVFRPGQPDASIYVVQDGLLELCLPGPDG 201 $\mathrm{LK} V \mathrm{VR}+\mathrm{E} \mathrm{L}+\mathrm{EL}+\mathrm{Q}$ GDY + $+\mathrm{G}+\mathrm{Y}++++\mathrm{G}+\mathrm{L}+\mathrm{DG}$ Sbjct: 470 LKKVRIFADCEAGLLVELVLKLQPQVYSPGDYICKKGDIGREMYIIKEG--KLAVVADDG 527

Query: 202 KECVVKEVVPGD-SVNSLLSILDVITGHQHPQRTVSARAARDSTVIRLPVEAFSAVFTKY 260 $\mathrm{V}+\mathrm{VV} \mathrm{DS}+\mathrm{SIL}++\quad+\mathrm{RT}+++\mathrm{S}+\mathrm{L}+\mathrm{T}+\mathrm{Y}$

Sbjct: 528 ---VTQFVVLSDGSYFGEISILNIKGSKAGNRRTANIKSIGYSDLFCLSKDDLMEALTEY 584 
SPIQ00194/CNG1_BOVIN CGMP-GATED CATION CHANNEL PROTEIN (CYCLIC NUCIEOTIDE GATED

CHANNEL, PHOTORECEPTOR) (CYCLIC-NUCLEOTIDE-GATED CATION CHANNEL 1) (CNG CHANNEL 1) (CNG-1) (CNG1)

Length $=690$

Score $=41.0$ bits $(94)$, Expect $=0.012$

Identities $=32 / 123(268)$, Positives $=58 / 123(47 \%)$, Gaps $=6 / 123(48)$

Query: 142 LKNVRVLGHFEKPLFLELCRHMVFQRLGQGDYVFRPGQPDASIYVVQDGLLELCLPGPDG 201 $\mathrm{LK} V \mathrm{VR}+\mathrm{E} \mathrm{I}+\mathrm{EL}+\mathrm{Q} \mathrm{GDY}+\mathrm{G}+\mathrm{Y}++++\mathrm{G}+\mathrm{L}+\mathrm{DG}$

Sbjct: 472 LKKVRIFADCEAGLIVELVLKLQPQVYSPGDYICKKGDIGREMYIIKEG--KLAVVADDG 529

Query: 202 KECVVKEVVPGD-SVNSILSILDVITGHQHPQRTVSARAARDSTVLRLPVEAFSAVFTKY 260

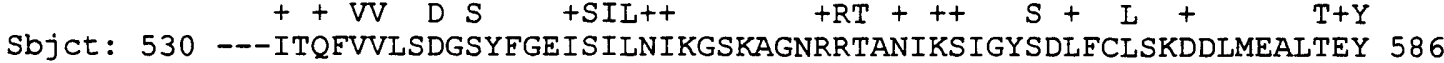

Query: 261 PES 263 $\mathrm{P}++$

Sbjct: 587 PDA 589

SP|P29974/CNG1_MOUSE CGMP-GATED CATION CHANNEL PROTEIN (CYCLIC NUCLEOTIDE GATED

CHANNEL, PHOTORECEPTOR) (CYCLIC-NUCLEOTIDE-GATED CATION CHANNEL 1) (CNG CHANNEL 1) (CNG-1) (CNG1)

Length $=684$

Score $=41.0$ bits $(94)$, Expect $=0.012$

Identities $=32 / 123(26 \%)$, Positives $=58 / 123(47 \%)$, Gaps $=6 / 123(4 \%)$

QuerY: 142 LKNVRVLGHFEKPLFLELCRHMVFQRLGQGDYVFRPGQPDASIYVVQDGLLELCLPGPDG 201 LK VR+ $E I+E L+Q$ GDY + + G +Y++++G +I + DG

Sbjct: 466 LKKVRIFADCEAGLIVELVLKLQPQVYSPGDYICKKGDIGREMYIIKEG--KLAVVADDG 523

QuerY: 202 KECVVKEVVPGD-SVNSLISILDVITGHQHPQRTVSARAARDSTVLRLPVEAFSAVFTKY 260

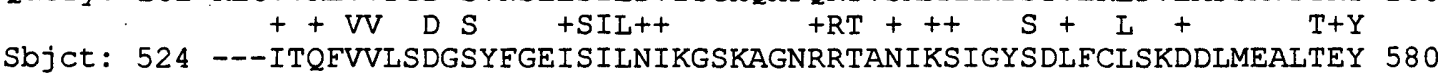

Query: 261 PES 263

Sbjct: 581 PDA 583

SP|Q62927/CNG1_RAT CGMP-GATED CATION CHANNEL PROTEIN (CYCLIC NUCLEOTIDE GATED CHANNNEL, PHOTORECEPTOR) (CYCLIC-NUCLEOTIDE-GATED CATION CHANNEL 1) (CNG CHANNEL 1) (CNG-1) (CNG1) Length $=683$

Score $=41.0$ bits $(94)$, Expect $=0.012$

Identities $=32 / 123(26 \%)$, Positives $=58 / 123(47 \%)$, Gaps $=6 / 123(4 \%)$

Query: 142 LKNVRVLGHFEKPLFLELCRHMVFQRLGQGDYVFRPGQPDASIYVVQDGLLELCLPGPDG 201 $\mathrm{LK} V R+\mathrm{E} \mathrm{L}+\mathrm{EL}+\mathrm{Q}$ GDY + G $+\mathrm{Y}++++\mathrm{G}+\mathrm{L}+\mathrm{DG}$

Sbjct: 465 LKKVRIFADCEAGLLVELVLKLQPQVYSPGDYICKKGDIGREMYIIKEG--KLAVVADDG 522

Query: 202 KECVVKEVVPGD-SVNSLISILDVITGHQHPQRTVSARAARDSTVLRLPVEAFSAVFTKY 260 $++\mathrm{VV}$ D S +SIL + + +RT + + $\mathrm{S}+\mathrm{L}+\quad \mathrm{T}+\mathrm{Y}$ Sbjct: 523 ---ITQFVVLSDGSYFGEISILNIKGSKAGNRRTANIKSIGYSDLFCLSKDDLMEALTEY 579

Query: 261 PES 263

Sbjct: 580 PDA 582 
SP/Q28279/CNGI_CANEA CGMP-GATED CATION CHANNEL PROTEIN (CYCLIC NUCLEOTIDE GATED

CHANNEL, PHOTORECEPTOR) (CYCLIC-NUCLEOTIDE-GATED CATION CHANNEL 1) (CNG CHANNEL 1) (CNG-1) (CNG1)

Length $=691$

Score $=41.0$ bits $(94)$, Expect $=0.012$

Identities $=32 / 123(26 \%)$, Positives $=58 / 123(47 \%)$, Gaps $=6 / 123\left(4 \frac{8}{8}\right)$

Query: 142 LKNVRVLGHFEKPLFLELCRHMVFQRLGQGDYVFRPGQPDASIYVVQDGLLELCLPGPDG 201 LK VR+ $E$ L +EL $+Q$ GDY + + + $++++G+I+D G$ Sbjct: 473 LKKVRIFADCEAGLIVELVLKLQPQVYSPGDYICKKGDIGREMYIIKEG--KLAVVADDG 530 QuerY: 202 KECVVKEVVPGD-SVNSLLSILDVITGHQHPQRTVSARAARDSTVLRLPVEAFSAVFTKY 260

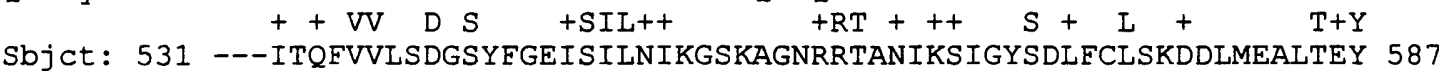
Query: 261 PES 263

Sbjct: 588 PDA 590 SP / 003043/KGP2_DROME CGMP-DEPENDENT PROTEIN KINASE, ISOZYME 2 FORMS T1/T3
(CGK)

(PROTEIN FORAGING)

Length $=1088$

Score $=40.6$ bits (93), Expect $=0.016$

Identities $=37 / 173(218)$, Positives $=76 / 173(43 \%)$, Gaps $=16 / 173$ (98)

QuerY: 487 AGTIIARQGDQDVSLHFVLWGCLHVYQRMIDKAEDVCLFVAQPGELVGQLAVLTGEPLIF 546 $\mathrm{A}+\mathrm{I}++\mathrm{GD}++\mathrm{G}+\mathrm{V}+\mathrm{E} \mathrm{L} \quad+++\mathrm{G}+\mathrm{LA}+\mathrm{L}$

Sbjct: 543 AKNLIIKEGDVGSIVYVMEDGRVEVSR------EGKYLSTLSGAKVLGELAILYNCQRTA 596

Query: 547 TLRAQRDCTFLRISKSDFYEIMRAQPSVVLSAAHTVAARMSPFVRQM--------DFAID 598 $\mathrm{T}+\mathrm{A}+\mathrm{C} \mathrm{I}+\mathrm{F}$ IM $+++\mathrm{A}+++\mathrm{P}+++$ Sbjct: 597 TITAITECNLWAIERQCFQTIMM-RTGLIRQAEYSDFLKSVPIFKDLAEDTLIKISDVLE 655 Query: 599 WTAVEAGRALYRQGDRSDCTYIVLNGRLRSVI-QRGSGKKELVGEYGRGDLIG 650 $\mathrm{T}+\mathrm{G}+\mathrm{RQG}$
Sbjet: 656 ETHYQRGDHIVRQGARGDTFFIISKGKVRVTIKQQDTQEEKFIRMLGKGDFFG 708

\section{SP|P46148|ETRA SHEPU ELECTRON TRANSPORT REGULATOR A Length $=250$}

Score $=40.2$ bits $(92)$, Expect $=0.020$

Identities $=19 / 108(17 \%)$, Positives $=54 / 108(49 \%)$, Gaps $=1 / 108(0 \%)$

Query: 602 VEAGRALYRQGDRSDCTYIVLNGRLRSVIQRGSGKKELVGEYGRGDLIGVVEALTRQPRA 661

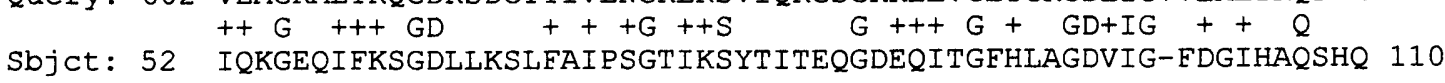

QuerY: 662 TTVHAVRDTELAKLPEGTLGHIKRRYPQVVTRLIHLLSQKILGNLQQL 709

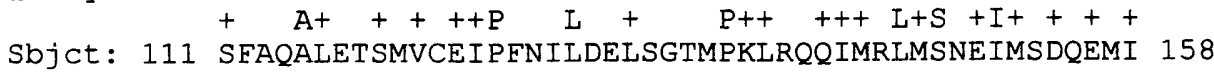


SP|P32023|KGP3_DROME CGMP-DEPENDENT PROTEIN KINASE, ISOZYME 2 FORMS T2/CD5 (CGK)

(PROTEIN FORAGING)

Length $=933$

Score $=40.2$ bits (92), Expect $=0.020$

Identities $=29 / 129(22 \%)$, Positives $=60 / 129(46 \%)$, Gaps $=10 / 129$ (7\%)

Query: 531 ELVGQLAVLTGEPLIFTLRAQRDCTFLRISKSDFYEIMRAQPSVVLSAAHTVAARMSPFV 590 $+++G+L A+L \quad T+A+C \quad I+F I M+++A+++P$

Sbjct: 426 KVLGELAILYNCQRTATITAITECNLWAIERQCEQTIMM-RTGLIRQAEYSDFLKSVPIF 484

Query: 591 RQM-------DFAIDWTAVEAGRALYRQGDRSDCTYIVLNGRLRSVI-QRGSGKKELVG 641 $++\quad++\mathrm{T}+\mathrm{G}+\mathrm{RQG} \mathrm{R} \mathrm{D}+\mathrm{I}+\mathrm{G}++\mathrm{R} \mathrm{I} \mathrm{Q}+++++$

Sbjct: 485 KDLAEDTLIKISDVLEETHYQRGDHIVRQGARGDTFFIISKGKVRVTIKQQDTQEEKFIR 544

Query: 642 EYGRGDLIG 650

G+GD G

Sbjct: 545 MLGKGDFFG 553

SP|P23619|HLYX_ACTPL REGULATORY PROTEIN HLYX

Length $=240$

Score $=39.9$ bits $(91)$, Expect $=0.027$

Identities $=20 / 108(188)$, Positives $=54 / 108$ (498), Gaps $=1 / 108(08)$

Query: 602 VEAGRALYRQGDRSDCTYIVLNGRLRSVIQRGSGKKELVGEYGRGDLIGVVEALTRQPRA 661

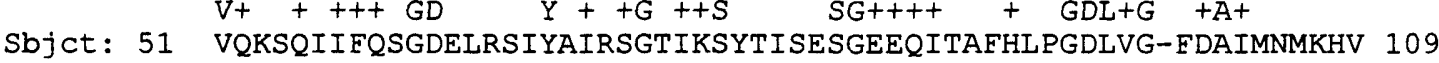

Query: 662 TTVHAVRDTELAKLPEGTLGHIKRRYPQVVTRLIHLLSQKILGNLQQL 709

$\begin{array}{ccc}\text { A }+++++P & \text { L }++ \text { P }+++++\mathrm{L}+\mathrm{S}+\mathrm{I}+++ \\ \text { Sbjct } & 110 \text { GEAQALETSMICEIPFDILDDLAGKMPKIRHQIMRLMSNEIKSDQEMI } 157\end{array}$

SP|Q14028/CNG4 HUMAN CYCLIC-NUCLEOTIDE-GATED CATION CHANNEL 4 (CNG CHANNEI 4) (CNG-4) (CNG4) (CYCLIC NUCLEOTIDE-GATED CATION CHANNEL MODULATORY SUBUNIT)

Length $=909$

Score $=39.9$ bits $(91)$, Expect $=0.027$

Identities $=34 / 117(29 \%)$, Positives $=56 / 117(47 \%)$, Gaps $=14 / 117(11 \%)$

Query: 155 LFLELCRHMVFQRLGQ-------GDYVFRPGQPDASIYVVQDGLLELCLPGPDGKECVV 206 $L F \quad R M+F \quad L+D Y V+G+\quad+Y++Q G+++L$ GPDGK $+V$ Sbjct: 620 LFQGCDRQMIFDMLKRLRSVVYLPNDYVCKKGEIGREMYIIQAGQVQV-LGGPDGKSVLV 678

Query: 207 KEVVPGDSVNSLLSILDVITGHQHPQRTVSARAARDSTVLRLPVEAFSAVFTKYPES 263 $+\mathrm{SV}+\mathrm{S}+\mathrm{LV} \mathrm{G}++\mathrm{RT}+\mathrm{A}++\mathrm{L}+++$ YPES Sbjct: 679 --TLKAGSVFGEISLLAVGGGN---RRTANVVAHGFTNLFILDKKDLNEILVHYPES 730

SP/Q28181/CNG4_BOVIN 240K PROTEIN OF ROD PHOTORECEPTOR CNG-CHANNEI (CONTAINS: GLUTAMIC ACID-RICH PROTEIN (GARP) AND CYCIIC-NUCLEOTIDE-GATED CATION CHANNEL 4 (CNG CHANNEL 4) (CNG-4) (CYCLIC NUCLEOTIDE-GATED CATION CHANNEL MODULATORY SUBUNIT)) Length $=1394$

Score $=39.9$ bits $(91)$, Expect $=0.027$

Identities $=34 / 117(29 \%)$, Positives $=56 / 117(47 \%)$, Gaps $=14 / 117$ (11\%)

Query: 155 LFLELCRHMVFQRLGQ-------GDYVFRPGQPDASIYVVQDGLLELCLPGPDGKECVV 206 $\mathrm{LF} \quad \mathrm{R} \mathrm{M}+\mathrm{F} \mathrm{L}+\mathrm{DYV}+\mathrm{G}+\quad+\mathrm{Y}++\mathrm{Q} \mathrm{G}+++\mathrm{I}$ GPDGK $+\mathrm{V}$ Sbjct : 1076 LFQGCDRQMI FDMLKRLRSVVYLPNDYVCKKGEIGREMYIIQAGQVQV-LGGPDGKSVLV 1134 
Query: 207 KEVVPGDSVNSLLSILDVITGHQHPQRTVSARAARDSTVLRLPVEAFSAVFTKYPES 263 $+\mathrm{SV}+\mathrm{S}+\mathrm{L} \mathrm{V} \mathrm{G+}+\mathrm{RT}+\mathrm{A}++\mathrm{L}+++$ YPES Sbjct: 1135 --TLKAGSVFGEISLLAVGGGN---RRTANVVAHGFTNLFILDKKDLNEILVHYPES 1186

SP/Q03611/YN82_CAEEL HYPOTHETICAL 88.6 KD PROTEIN ZC84.2 IN CHROMOSOME III Length $=772$

Score $=39.5$ bits $(90)$, Expect $=0.035$

Identities $=31 / 122(25 \%)$, Positives $=54 / 122(43 \%)$, Gaps $=4 / 122(38)$

Query: 142 LKNVRVLGHFEKPLFLELCRHMVFQRLGQGDYVFRPGQPDASIYVVQDGLLELCLPGPDG 201 $\mathrm{L}+\mathrm{VR}+\mathrm{E} \mathrm{L} \mathrm{EL}+\mathrm{Q} \mathrm{GD}+++\mathrm{G}+\mathrm{Y}+\mathrm{V}+\mathrm{G} \mathrm{L}++\quad \mathrm{DG}$ Sbjct: 519 LRKVRIFQDCEAGLLAELVLKLQLQVFSPGDFICKKGDIGREMYIVKRGRLQVV--DDDG 576

QuerY: 202 KECVVKEVVPGDSVNSLLSILDVITGHQHPQRTVSARAARDSTVLRLPVEAFSAVFTKYP 261

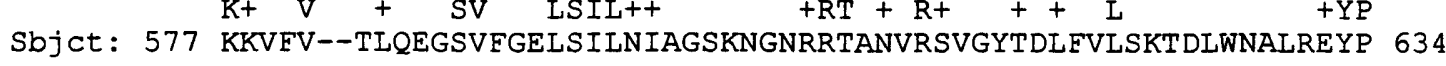
Query: 262 ES 263 Sbjct: 635 DA 636

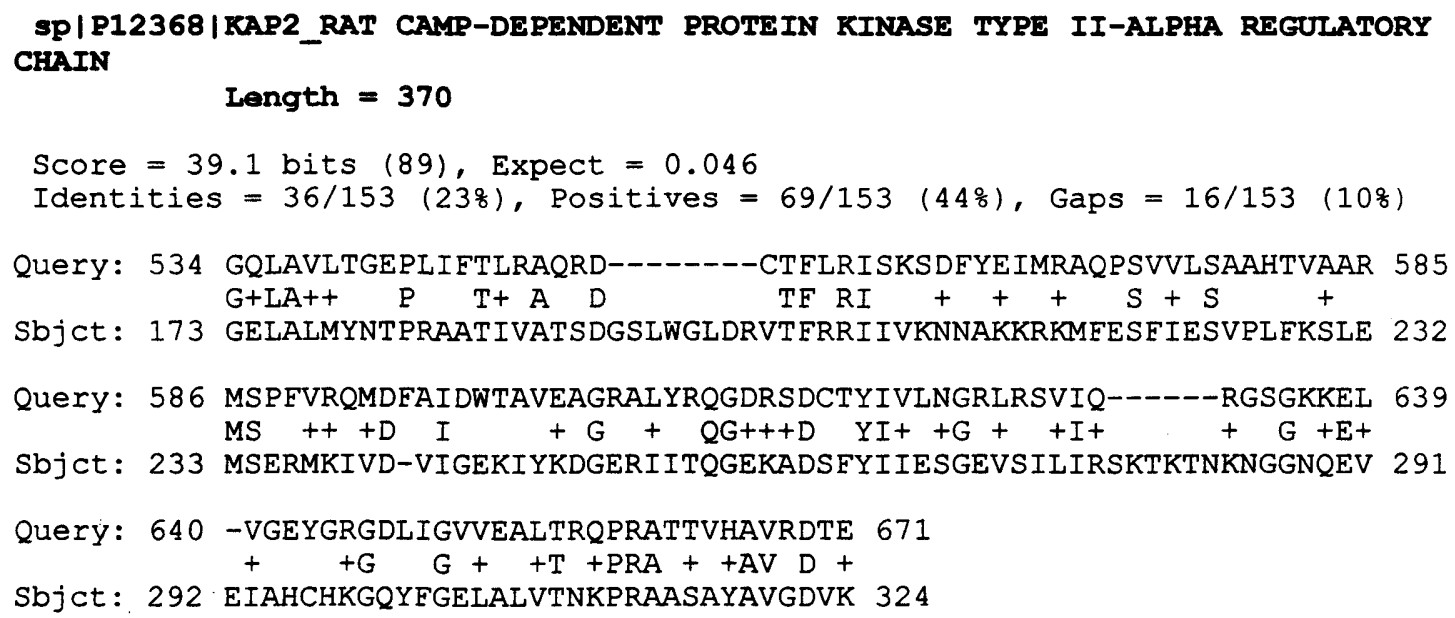

\title{
Appendix 4 5' RACE and HOL4.2 DNA sequences
}

\section{a28}

\author{
1 AACAAAAGCT GGAGTCCACC GCGGTGGCGG CCGCCCGGGC AGGTGGCGCC \\ 51 ACCATCGgtC CCGgAgtCCC AgtgatgCtC tgtgCCAtAg AgCCCCCAtA \\ 101 ACTTCACTAC TACGTGATAG TAAATCCCCG GCAAAAACCA GCAGCGCCTT \\ 151 GCAAGCCCAC GCCACCCCAA GCATCCCAGG ACTCTTCTGA AACGACTCCG \\ 201 GGCTACCAGA TCGGCCGTCC AGCTGGAATC AACCGATGGA GGCTCCGCTG \\ 251 CAAACTgGAA TGgTGCTTGg CGTGATGATC GgGGCCGGAG TGgCGgtgGT \\ 301 GGTCACGGCC GTGCTCATCC TCCTGGTGGT GCGGAGGCTG CGAGTGCCAA \\ 351 AAACCCCAGC CCCGGATGGC CCCCGGTATC GGTTCCGGAA GAGGGACAAA
}


401 GTGCTCTtCT AtgGCCGGAA GATtATGCGg AAGGTGTCAC AATCCACCTC

451 CTCCCTCGTG GATACCTCTG TCTCCGCCAC CTCCCGGCCA CGCATGAGGA

501 AgAAACTGAA GATGCTCAAC ATTGCCAAGA AGATCCTGCG CATCCAGAAA

551 GAGACGCCCA CGCTGCAGCG GAAGGAGCCC CCGCCCGCAG TGCTAGAAGC

601 TGACCTGACC GAGGGCGACC TGGCTAACTC CCATCTGCCC TCTGAAGTGC

651 TTTATATGCT CAAGAACGTC CGGGTGCTGG GCCACTTCGA GAAGCCACTC

701 TTCCTGGAGC TCTGCCGCCA CATGGTCTTC CAGCGGCTGG GCCAGGGTGA

751 CTACGTCTTC CGGCCGGGCC AGCCAGATGC CAGCATCTAC GTGGTGCAGG

801 ACGGGCTGCT GGAGCTCTGT CTGCCAGGGC CTGACGGGAA GGAGTGTGTG

851 GTGAAGGAAG TGGTTCCTGG GGACAGCGTC AACAGCCTTC TCAGCATCCT

901 GGATGTCATC ACCGGTCACC AGCATCCCCA GCGGACCGTG TCTGCCCGGG

951 CGGCCCGGGA CTCCACGGTG CTGCGCCTGC CGGTGGAAGC ATTCTCCGCG

1001 GTCTTCACCA AGTACCCGGA GAGCTTGGTG CGGGTCGTGC AGATCATCAT

1051 GGTGCGGCTG CAGCGAGTCA CCTTACTGGC ACTGCACAAC TACCTGGGTC

1101 TGACCAATGA GCTCTTCAGC CACGAGATCC AGCCCCTGCG TCTGTTCCCC

1151 AGCCCCGGCC TCCCAACTCG CACCAGCCCT GTGCGGGGCT CCAAGAGAAT

1201 GGTCAGCACC TCAGCTACAG ACGAGCCCAG GGAGACCCCA GGGCGGCCAC

1251 CCGATCCCAC CGGGGCCCCG CTGCCTGGAC CTACAGGGGA CCCTGTGAAG

1301 CCCACATCCC TGGAAACCCC CTCGGCCCCT CTGCTGAGCC GCTGCGTCTC

1351 CATGCCAGGG GACATCTCAG GCTTGCAGGG TGGCCCCCGC TCCGACTTCG

1401 ACATGGCCTA TGAGCGTGGC CGGATCTCCG TGTCCCTGCA GGAAGAGGCC

1451 TCCGGGGGGT CCCTGGCAGC CCCCGCTCGG ACCCCCACTC AGGAGCCTCG

1501 TGAGCAGCCG GCAGGCGCCT GTGAATACAG CTACTGTGAG GATGAGTCGG

1551 CCACTGGTGG CTGCCCTTTC GGGCCCTACC AGGGCCGCCA GACCAGCAGC

1601 ATCTTCGAGG CAGCAAAGCA GGAGCTGGCC AAGCTGATGC GGATTGAGGA

1651 CCCCTCCCTC CTGAACAGCA GAGTCTTGCT GCACCACGCC AAAGCTGGCA

1701 CCATCATTGC CCGCCAGGGA GACCAGGACG TGAGCCTGCA CTTCGTGCTC

1751 TGGGGCTGCC TGCACGTGTA CCAGCGCATG ATCGACAAGG CGGAGGACGT

1801 GTGCCTGTTC GTAGCGCAGC CCGGGGAACT GGTGGGGCAG CTGGCGGTGC

1851 TCACTGGCGA ACCTCTCATC TTCACACTGC GAGCCCAACG CGACTGCACC

1901 TTCCTGCGGA TCTCCAAGTC CGACTTCTAT GAGATCATGC GCGCACAGCC

1951 CAGTGTGGTG CTGAGTGCGG CGCACACGGT GGCAGCCAGG ATGTCGCCCT 
2001 TCGTGCGCCA GATGGACTTC GCCATCGACT GGACTGCAGT GGAGGCGGGA 2051 CGCGCGCTGT ACAGGCAGGG CGACCGCTCC GACTGCACTT ACATCGTGCT

2101 CAATGGGCGG CTGCGTAGCG TGATCCAGCG AgGCAGTGGC AAGAAGGAGC 2151 TGGTGGGCGA GTACGGCCGC GGCGACCTCA TCGGCGTGGT GGAGGCACTG 2201 ACCCGGCAGC CGCGAGCCAC GACGGTGCAC GCGGTGCGCG ACACGGAGCT 2251 GGCCAAGCTT CCCGAGGGCA CCTTGGGTCA CATCAAACGC CGGTACCCGC 2301 AGGTCGTGAC CCGCCTTATC CACCTACTGA GCCAGAAAAT TCTAGGGAAT 2351 TTGCAGCAGC TGCAAGGACC CTTCCCAGCA GGCTCTGGGT TGGGTGTGCC 2401 CCCACACTCG GAACTCACCA ACCCAGCCAG CAACCTGGCA ACTGTGGCAA 2451 TCCTGCCTGT GTGTGCTGAG GTCCCCATGG TGGCCTTCAC GCTGGAGCTG 2501 CAGCACGCCC TGCAGGCCAT CGGTCCGACG CTACTCCTTA ACAGTGACAT 2551 CATCCGGGCA CGCCTGGGGG CCTCCGCACT GGATAGCATC CAAGAGTTCC 2601 GGCTGTC

\section{HOL 4.2}

1 GCGGGGAATT TGCAGCAGCT GCAAGGACCC TTCCCAGGCT CTGGGTTGGG 51 TGTGCCCCCA CACTCGGAAC TCACCAACCC AGCCAGCAAC CTGGCAACTG 101 TGGCAATCCT GCCTGTGTGT GCTGAGGTCC CCATGGTGGC CTTCACGCTG 151 GAGCTGCAGC ACGCCCTGCA GGCCATCGGT CCGACGCTAC TCCTTAACAG 201 TGACATCATC CGGGCACGCC TGGGGGCCTC CGCACTGGAT AGCATCCAAG 251 AGTTCCGGCT GTCAGGGTGG CTGGCCCAGC AGGAGGATGC ACACCGTATC 301 GTACTCTACC AGACGGACGC CTCGCTGACG CCCTGGACCG TGCGCTGCCT 351 GCGACAGGCC GACTGCATCC TCATTGTGGG CCTGGGGGAC CAGGAGCCTA 401 CCCTCGGCCA GCTGGAGCAG ATGCTGGAGA ACACGGCTGT GCGCGCCCTT 451 AAGCAGCTAG TCCTGCTCCA CCGAGAGGAG GGCGCGGGCC CCACGCGCAC 501 CGTGGAGTGG CTAAATATGC GCAGCTGGTG CTCGGGGCAC CTGCACCTGC 551 GCTGTCCGCG CCGCCTCTTT TCGCGCCGCA GCCCTGCCAA GCTGCATGAG 601 CTCTTCGACA AGGTTTTCTC CAGGCGCGCG GACCGGCACA GCGACTTCTC 701 CCGCTTGGCG AGGGTGCTCA CGGGGAACAC CATTGCCCTT GTGCTAGGCG 751 GGGGCGGGGC CAGGGGCTGC TCGCACATCG GAGTACTAAA GGCATTAGAG 801 GAGGCGGGGG TCCCCGTGGA CCTGGTGGGC GGCACGTCCA TTGGCTCTTT 901 CATCGGAGCG TTGTACGCGG AGGAGCGCAG CGCCAGCCGC ACGAAGCAGC 
951 GGGCCCGGGA GTGGGCCAAG AGCATGACTT CGGTGCTGGA ACCTGTGTTG 1001 GACCTCACGT ACCCAGTCAC CTCCATGTTC ACTGGGTCTG CCTTTAACCG 1051 CAGCATTCAT CGGGTCTTCC AGGATAAGCA GATTGAGGAC CTGTGGCTGC 1101 CTTACTTCAA CGTGACCACA GATATCACCG CCTCAGCCAT GCGAGTCCAC 1151 AAAGATGGCT CCCTGTGGCG GTACCTGCGC GCCAGCATGA CGCTGTCGGG 1201 CTACCTGCCC CCGNTGTGCG ACCCCAAGGA CGGGCACCTA CTCATGGATG 1251 GCGGCTACAT CAACAATCTG CCAGCGGACA TCGCCCGCAG CATGGGTGCC 1301 AAAACGGTCA TCGCCATTGA CGTGGGRAGC CAGGATGAGA CGGACCTCAG 1351 CACCTACGGG GACAGCCTGT CCGGCTGGTG GCTGCTGTGG AAGCGGCTGA 1401 ATCCCTGGGC TGACAAGGTA AAGGTTCCAG ACATGGCTGA AATCCAGTCC 1451 CGCCTGGCCT ACGTGTCCTG TGTGCGGCAG CTAGAGGTTG TCAAGTCCAG 1501 CTCCTACTGC GAGTACCTGC GCCCGCCCAT CGACTGCTTC AAGACCATGG 1551 ACTTTGGGAA GTTCGACCAG ATCTATGATG TGGGCTACCA GTACGGGAAG 1601 GCGGTGTTTG GAGGCTGGAG CCGTGGCAAC GTCATTGAGA AAATGCTCAC 1651 AGACCGGCGG TCTACAGACC TTAATGAGAG CCGCCGTGCA GACGTGCTTG 1701 CCTTCCCAAG CTCTGGCTTC ACTGACTTGG CAGAGATTGT GTCCCGGATT 1751 GAGCCCCCCA CGAGCTATGT CTCTGATGGC TGTGCTGACG GAGAGGAGTC 1801 AGATTGTCTG ACAGAGTATG AGGAGGACGC CGGACCCGAC TGCTCGAGGG 1851 ATGAAGGGGG GTCCCCCGAG GGCGCAAGCC CCAGCACTGC CTCCGAGATG 1901 GAGGARGAGA AGTCGATTCT CCGGCAACGA CGCTGTCTGC CCCAGGAGCC 1951 GCCCGGCTCA GCCACAGATG CCTGAGGACC TCGACAGGGG TCACCCCCTC 2001 CCTCCCACCC CTGGACTGGG CTGGGGGTGG CCCCGTGGGG GTAGCTCACT 2051 CCCCCTCCTG CTGCTATGCC TGTGACCCCC GCGGCCCACA CACTGGACTG 2101 ACCTGCCCTG AGCGGGGATG CAGTGTTGCA CTGATGACTT GACCAGCCCC 2150 TCCCCCAATA AACTCGCCTC TTGGAAAAAA AAAAAAAAAA AAAAAA 


\section{Translation of the full a28-HOL4.2 sequence}

(residues mentioned in Chapter 4 shown in bold) 1

a28HOL4 AACAAAAGCT GGAGTCCACC GCGGTGGCGG CCGCCCGGGC AGGTGGCGCC ntelpep .T..K..A.. G..V..H..R ..G..G..G. .R..P..G.. R..W..R..H

$51 \quad 100$

a28HOL4 ACCATCGGTC CCGGAGTCCC AGTGATGCTC TGTGCCATAG AGCCCCCATA ntelpep ..H..R..S. .R..S..P.. S..D..A..L ..C..H..R. .A..P..I..

$101 \quad 150$ ntelpep T..S..I..L ..R..D..S. .K..S..P.. A..K..T..S ..S..A..L

151200

a28HOL4 GCAAGCCCAC GCCACCCCAA GCATCCCAGG ACTCTTCTGA AACGACTCCG

ntelpep .Q..A..H.. A..T..P..S .I..P..G. .L..F..*.. N..D..S..G

$\begin{array}{ll}201 & 250 \\ \text { a28HOL4 } & \end{array}$

ntelpep ..L..P..D. .R..P..S.. S..W..N..Q ..P..M..E. .A..P..L.

251

a28HOL4 CAAACTGGAA TGGTGCTTGG CGTGATGATC GGGGCCGGAG TGGCGGTGGT

ntelpep Q..T..G..M ..V..L..G. .V..M..I.. G..A..G..V ..A..V..V.

301

a28HOL4 GGTCACGGCC GTGCTCATCC TCCTGGTGGT GCGGAGGCTG CGAGTGCCAA

ntelpep .V..T..A.. V..L..I..I ..L..V..V. .R..R..L.. R..V..P..K

$351 \quad 400$

a28HOL4 AAACCCCAGC CCCGGATGGC CCCCGGTATC GGTTCCGGAA GAGGGACAAA

ntelpep ..T..P..A. .P..D..G.. P..R..Y.R ..F..R..K. .R..D..K..

a28HOL4 GTGCTCTTCT ATGGCCGGAA GATTATGCGG AAGGTGTCAC AATCCACCTC

ntelpep V..L..F..Y ..G..R..K. .I..M..R.. K..V..S..Q ..S..T..S.

$451 \quad 500$

a28HOL4 CTCCCTCGTG GATACCTCTG TCTCCGCCAC CTCCCGGCCA CGCATGAGGA ntelpep .S..L..V.. D..T..S..V ..S..A..T. .S..R..P.. R..M..R..K

ntelpep ..K..L..K. .M..L..N.. I..A..K..K ..I..L..R. .I..Q..K..

$551 \quad 600$

a28HOL4 GAGACGCCCA CGCTGCAGCG GAAGGAGCCC CCGCCCGCAG TGCTAGAAGC

ntelpep E..T..P.T ..L..Q.R. .K..E..P.. P..P..A.V ..L.E..A.

$601 \quad 650$

a28HOL4 TGACCTGACC GAGGGCGACC TGGCTAACTC CCATCTGCCC TCTGAAGTGC

ntelpep .D..I..T.. E..G.D..I ..A..N..S. .H.I..P.. S..E..V.I

651

a28HOL4 TTTATATGCT CAAGAACGTC CGGGTGCTGG GCCACTTCGA GAAGCCACTC

ntelpep ..Y..M..L. .K..N..V.. R..V..L..G ..H..F..E. .K..P..L..

701

a28HOL4 TTCCTGGAGC TCTGCCGCCA CATGGTCTTC CAGCGGCTGG GCCAGGGTGA

ntelpep F..L..E..L ..C..R..H. .M..V..F.. Q..R..L..G .Q..G..D. 
751

a28HOL 4

CTACGTCTTC CGGCCGGGCC AGCCAGATGC CAGCATCTAC GTGGTGCAGG

ntelpep .Y..V..F.. R..P..G..Q ..P.D..A. .S..I.Y.. V..V....D

801

a28HOL 4

ACGGGCTGCT GGAGCTCTGT CTGCCAGGGC CTGACGGGAA GGAGTGTGTG

ntelpep

..G..L..L. .E..L..C.. L..P..G..P ..D..G..K. .E..C..V..

851

900

a28HOL 4

GTGAAGGAAG TGGTTCCTGG GGACAGCGTC AACAGCCTTC TCAGCATCCT

ntelpep

V..K..E..V ..V..P..G. .D..S..V.. N..S..L..L ..S..I..L.

901

950

a28HOL 4

GGATGTCATC ACCGGTCACC AGCATCCCCA GCGGACCGTG TCTGCCCGGG

ntelpep

951

1000

a28HOL4 CGGCCCGGGA CTCCACGGTG CTGCGCCTGC CGGTGGAAGC ATTCTCCGCG ntelpep ..A..R..D. .S..T..V.. L..R..L..P ..V..E..A. .F..S..A..

1001

1050

a28HOL4

GTCTTCACCA AGTACCCGGA GAGCTTGGTG CGGGTCGTGC AGATCATCAT

ntelpep

1051

1100

a28HOL 4

ntelpep

CAGCGAGTCA CCTTACTGGC ACTGCACAAC TACCTGGGTC .V..R..L.. Q..R..V..T ..L..L..A. .L..H..N.. Y..L..G..L

1101

1150

a28HOL 4

TGACCAATGA GCTCTTCAGC CACGAGATCC AGCCCCTGCG TCTGTTCCCC ntelpep ..T..N..E. .L..F..S.. H..E..I..Q ..P.L..R. .L..F..P.

1151

1200

a28HOL 4 ntelpep

AGCCCCGGCC TCCCAACtCg CACCAGCCCt GtGCGGGGCT CCAAGAGAAT S..P..G.L ..P.T.R. .T..S..P. V..R..G..S ..K..R..M.

1201

1250

a28HOL4

GGTCAGCACC TCAGCTACAG ACGAGCCCAG GGAGACCCCA GGGCGGCCAC ntelpep

.V..S..T.. S..A..T.D ..E..P.R. .E.T..P.. G.R..P.P

1251

1300

a28HOL 4 CCGATCCCAC CGGGGCCCCG CTGCCTGGAC CTACAGGGGA CCCTGTGAAG

ntelpep

1301

1350

a28HOL 4

CCCACATCCC TGGAAACCCC CTCGGCCCCT CTGCTGAGCC GCTGCGTCTC

ntelpep

P..T..S..L ..E..T..P. .S..A..P.. L..L..S.R ....V..S.

1351

1400

a28HOL 4

CATGCCAGGG GACATCTCAG GCTTGCAGGG TGGCCCCCGC TCCGACTTCG

ntelpep

1401

1450

a28HOL4 ACATGGCCTA TGAGCGTGGC CGGATCTCCG TGTCCCTGCA GGAAGAGGCC ntelpep ..M..A..Y. .E..R..G.. R..I..S..V ..S..I..Q. .E..E..A..

1451

a28HOL 4 TCCGGGGGGT CCCTGGCAGC CCCCGCTCGG ACCCCCACTC AGGAGCCTCG ntelpep .E..Q..P..A..G..A..C ..E.Y..S. .Y..C..E..D.E..S.A 
a28HOL4 CCACTGGTGG CTGCCCTTTC GGGCCCTACC AGGGCCGCCA GACCAGCAGC ntelpep ..T..G..G. .C..P..F.. G..P.Y..Q ..G..R..Q. .T..S..S..

1601

a28HOL4 ATCTTCGAGG CAGCAAAGCA GGAGCTGGCC AAGCTGATGC GGATTGAGGA ntelpep I..F..E..A..A..K..Q. .E..L..A..K..L..M..R ..I..E..D.

a28HOL 4 CCCCTCCCTC CTGAACAGCA GAGTCTTGCT GCACCÄCGCC AAAGCTGGCA ntelpep .P..S..L.. L..N..S..R ..V..L..L. .H..H..A.. K..A..G.T 1701

a28HOL 4 ntelpep

a28HOL 4 ntelpep

a28HOL 4 ntelpep

a28HOL 4 ntelpep

a28HOL 4 ntelpep

a 28 HOL 4 ntelpep

a28HOL 4 ntelpep

a28HOL 4 ntelpep

a28HOL 4 ntelpep

a28HOL 4 ntelpep

a 28 HOL 4 ntelpep

a28HOL 4 ntelpep

2251

TTCCTGCGGA TCTCCAAGTC CGACTTCTAT GAGATCATGC GCGCACAGCC

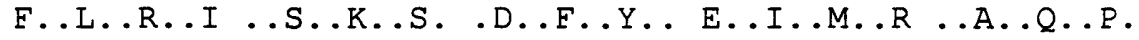

1951 2000

CAGTGTGGTG CTGAGTGCGG CGCACACGGT GGCAGCCAGG ATGTCGCCCT .S..V..V.. L..S..A..A ..H.T..V. .A..A..R.. M..S..P..F

2001 2050

TCGTGCGCCA GATGGACTTC GCCATCgaCT GGACTGCAGT GGAGGCGGGA ..V..R..Q. .M..D..F.. A..I..D..W ..T.A..V. .E..A..G..

2051 2100

CGCGCGCTGT ACAGGCAGGG CGACCGCTCC GACTGCACTT ACATCGTGCT R..A..I..Y ..R..Q..G. .D..R..S.. D..C.T.Y ..I..V.I.

2101

2150

CAATGGGCGG CTGCGTAGCG TGATCCAGCG AGGCAGTGGC AAGAAGGAGC .N..G..R.. L..R..S..V ..I..Q..R. .G..S..G.. K..K..E..L

2151 2200 TGGTGGGCGA GTACGGCCGC GGCGACCTCA TCGGCGTGGT GGAGGCACTG ..V..G..E. .Y..G..R..G..D..L.I ..G..V..V. .E..A..L..

ACCCGGCAGC CGCGAGCCAC GACGGTGCAC GCGGTGCGCG ACACGGAGCT T.R..Q.P \#R..A.T. .T.V..H.. A..V.R..D . T.E..L.

$$
2300
$$
.A..K..L.. P..E..G..T ..L..G..H..I..K..R..R..Y..P..Q

a28HOL4 AGGTCGTGAC CCGCCTTATC CACCTACTGA GCCAGAAAAT TCTAGGGAAT ntelpep ..V..V..T. .R..L..I.. H..L..L..S ..Q..K..I. .L..G..N. 
a28HOL4 TTGCAGCAGC TGCAAGGACC CTTCCCAGCA GGCTCTGGGT TGGGTGTGCC ntelpep L..Q..Q..L ..Q..G..P. .F..P..A.. G..S..G..L ..G..V..P.

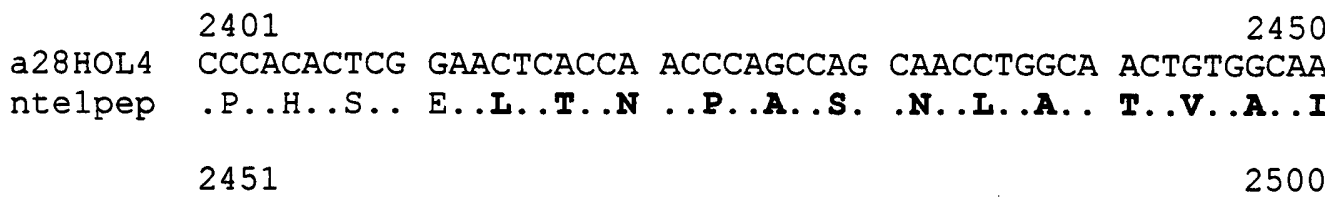
a28HOL4 TCCTGCCTGT GTGTGCTGAG GTCCCCATGG TGGCCTTCAC GCTGGAGCTG ntelpep ..I..P..V. .C..A..E.. V..P..M..V ..A..F..T. .L..E..I.. 2501

a28HOL4 CAGCACGCCC TGCAGGCCAT CGGTCCGACG CTACTCCTTA ACAGTGACAT ntelpep Q..H..A..L ..Q..A..I. .G..P.T.. L..L..L..N ..S..D..I.

a28HOL4 CATCCGGGCA CGCCTGGGGG CCTCCGCACT GGATAGCATC CAAGAGTTCC ntelpep .I..R..A.. R..L..G..A ..S..A..L. .D..S..I.. Q..E..F..R 26012650 a28HOL4 GGCTGTCAGG GTGGCTGGCC CAGCAGGAGG ATGCACACCG TATCGTACTC ntelpep ..L..S..G. .W..L..A.. Q..Q..E..D ..A..H..R. .I..V..I.. ntelpep Y..Q..T..D ..A..S..L. .T..P..W.. T..V..R..C ..L..R..Q. 2701 a28HOL4 GGCCGACTGC ATCCTCaTTG TGGGCCTGGG GGACCAGGAG CCTACCCTCG ntelpep .A..D..C.. I..L..I..V ..G..L..G. .D..Q..E.. P..T..L..G 2751 2800

a28HOL 4 GCCAgCTGGA GCAGATGCTG GAGAACACGG CTGTGCGCGC CCTTAAGCAg ntelpep ..Q..L..E. .Q..M..L.. E..N..T..A ..V..R..A. .I..K..Q.. 2801 a28HOL4 CTAgTCCTGC TCCACCgAgA gGAGGGCGCG GGCCCCACGC GCACCGTGGA ntelpep L..V..L..L ..H..R..E. .E..G..A.. G..P.T..R ..T..V..E.

a28HOL4 GTGGCTAAAT ATGCGCAGCT GGTgCTCGGG GCACCTGCAC CTGCgCTGTC ntelpep .W..L..N.. M..R..S..W ..C..S..G. .H..L..H.. L..R..C..P 2901 a28HOL4 CGCGCCGCCT CTTTTCGCGC CGCAGCCCTG CCAAGCTGCA TGAGCTCTTC ntelpep ..R..R..L. .F..S..R.. R..S..P..A ..K..L..H. .E..I..F.. $2951 \quad 3000$

a28HOL4 GACAAGGTTT TCTCCAGGCG CGCGGACCGG CACAGCgaCT TCTCCCGCTT ntelpep D..K..V..F ..S..R..R. .A..D..R.. H..S..D..F ..S..R..L. 3001

a28HOL4 GGCGAGGGTG CTCACGGGGA ACACCATTGC CCTTGTGCTA GGCGGGGGCG ntelpep .A..R..V.. L..T..G..N ..T..I.A. .L..V..L.. G..G..G..G 3051 a28HOL4 GGGCCAGGGG CTGCTCGCAC ATCGGAGTAC TAAAGGCATT AGAGGAGGCG ntelpep ..A..R..G. .C..S..H.. I..G..V..L ..K..A..L. .E..E..A..

a28HOL4 GGGGTCCCCG TGGACCTGGT GGGCGGCACG TCCATTGGCT CTTTCATCGG ntelpep G..V..P..V ..D..L..V. .G..G..T.. S..I..G..S ..F..I..G. 
a28HOL4 AGCGTTGTAC GCGGAGGAGC GCAGCGCCAG CCGCACGAAG CAGCGGGCCC ntelpep .A..L..Y..A..E..E..R ..S.A..S. .R..T.K.. Q..R..A..R

GGGAGTGGGC CAAGAGCATG ACTTCGGtgC TGGAACCTGT GTTGGACCTC

TCATCGgGTC TTCCAgGATA AgCAgAtTgA GGACCTGTgG CTGCCTTACT .H..R..V.. F..Q..D.K ..Q.I.E. .D..I..W.. I..P.Y..F

a28HOL 4 TCAACGTGAC CACAGATATC ACCGCCTCAg CCATGCgAGT CCACAAAGAT ntelpep ..N..V..T. .T..D..I.. T..A..S.A..M..R..V. .H..K..D.

a28HOL 4 ntelpep

a28HOL 4 ntelpep

a28HOL 4 ntelpep

a28HOL 4 ntelpep

a28HOL 4 ntelpep

a 28 HOL 4 ntelpep

a28HOL 4 ntelpep

a28HOL 4 ntelpep

a 28HOL 4 ntelpep

a28HOL 4 ntelpep

3401 3450 GGCTCCCTGT GGCGGTACCt GCGCGCCAGC ATGACgCTGT CGGGCTACCT G..S..L..W ..R..Y..L. .R..A..S.. M..T.L..S ..G..Y..L.

3451

3500 GCCCCGCTG TGCGACCCCA AgGACGGGCA CCTACTCATG GATGGCGGCT .P..P..L.. C..D..P..K ..D..G..H. .L..L..M.. D..G..G..Y

3501

3550

ACATCAACAA TCTGCCAGCG GACATCGCCC GCAGCATGGG TGCCAAAACG ..I..N..N. .I..P..A.. D..I..A..R ..S..M..G. .A..K..T.

3551 3600 GTCATCGCCA TTgACGTGGG GAGCCAGGAT gAGaCGGACC TCAGCACCTA V..I..A..I ..D..V..G. .S..Q..D. E..T.D..L ..S.T.Y.

CGGGGACAGC CTGTCCGGCT GGTGgCTgCT GTGGAAgCGG CTgAATCCCT .G..D..S.. L..S..G..W ..W..L..L. .W..K..R.. L..N..P.WW 3651 3700 GGGCTGACAA GGTAAAGGTT CCAGACATGG CTGAAATCCA GTCCCGCCTG ..A..D..K. .V..K..V.. P..D..M..A ..E..I.Q. .S.R..L..

GCCTACGTGT CCTGTGTgCG GCAGCTAGAG GTTGTCAAGT CCAGCTCCTA A..Y..V..S ..C..V..R. .Q..L..E..V..V..K..S ..S..S.Y.

3751

CTGCGAGTAC CTGCGCCCGC CCATCGACTG CTTCAAGACC ATGGACTTTG .C..E..Y.. L..R..P.P \#I..D..C. .F..K....M..D....G

GGAAGTTCGA CCAGATCTAT GATGTGGGCT ACCAGTACGG GAAGGCGGTG ..K..F..D. .Q..I.Y.. D..V..G.Y ..Q.Y..G. .K..A..V..

3851

TTTGGAGGCT GGAGCCGTGG CAACGTCATT GAGAAAATGC TCACAGACCG E..G..G..W ..S..R..G. .N..V..I.. E..K..M..L ..T.D.R. GTCTACA GACCTTAATG AGAGCCGCCG TGCAGACGTG CTTGCCTTCC .R..S..T.. D..I..N..E ..S..R..R. .A..D..V.. L..A..F..P 
a28HOL4 CAAGCTCTGG CTTCACTGAC TTGGCAGAGA TTGTGTCCCG GATTGAGCCC ntelpep ..S..S..G. .F..T..D.. L..A..E..I ..V..S..R. .I..E..P..

4001

a28HOL4 CCCACGAGCT ATGTCTCTGA TGGCTGTGCT GACGGAGAGG AGTCAGATTG ntelpep P..T.S..Y ..V..S..D. .G..C..A.. D..G.E.E ..S..D.C.

a28HOL4 TCTGACAGAG TATGAGgagG ACGCCgGACC CGACTGCTCG AgGGATGAAG ntelpep .L..T.E..Y..E.E..D ..A..G..P. .D..C..S..R..D.E..G

a28HOL 4 ntelpep

a28HOL 4 ntelpep

a28HOL 4 ntelpep

a28HOL 4 ntelpep

a28HOL 4 ntelpep

a28HOL 4 ntelpep

CCTGAGCGGG GATGCAGTGT TGCACTGATG ACTTGACCAG CCCCTCCCCC .L..S..G.. D..A..V..L ..H..*..*. .L..D..Q.. P..L..P.QQ

4401 4440

a28HOL4 AATAAACTCG CCTCTTGGAA AAAAAAAAAa IaAAAAAAAA ntelpep ..*..T..R. .L..L..E.. K..K..K..K ..K..K.. 


\section{References}

Abou-Donia M. B. (1981) Organophosphorus ester induced delayed neurotoxicity. Ann. Rev. Pharmacol. Toxicol. 21, 511-548

Aldridge W. N., (1954) Tricresylphosphate and cholinesterase. Biochem. J. 56 pp185-189

Altschul S. F., Madden T. L., Alejandro A., Schaumlffer A. A., Zhang J., Zhang Z., Miller W, and Lipman D. J., (1997) Gapped BLAST and PSI-BLAST: a New Generation of Protein Database Search Programs. Nucleic Acids Res. 25 pp3389-3402.

Argos P., (1989) Predicutions of Portein Structure from Gene and Amino Acid Sequences. IRL press. Protein Stucture a Practical Approach. Edited by Creinghton T. E. pp169-190

Auld V. J., Fetter R. D., Broadie K. B. and Goodman C. S., (1995) Gliotactin, a Novel Transmembrane Protein on Peripheral Glia, Is Required to Form the Blood-Nerve Barrier in Drosophila. Cell 81 pp757-767

Ayyub C., Paranjape J., Rodrigues V. and Siddiqi O., (1990) Genetics of Olfactory Behavior in Drosphila Melanogaster. J. Neurogenetics 6 pp243-262

Bitstrup P. L., Bonnell J. A. and Beckett A. G., (1953) Paralysis Following Poisoning by a New Organic phosphorus insecticide (mipafox) Brit. Med. j. 1 pp1068-69

Bouldin T. W. and Cavanagh J. B., Organophosphate Neuropathy: I. A TeasedFiber Study of the Spatio-Temporal Spread of Axonal Degeneration. Am. Ass. Pathologists 94(2) pp241-251

Brendel V., Bucher P., Nourbakhsh I., Blaisdell B. E. and Karlin S. (1992) Methods and Algorithms for Statistical Analysis of Protein Sequences. Proc. Natl. Acad. Sci. USA 89 pp2002-2006

Brenner S., (1988) The Molecular Evolution of Genes and Proteins: a Tale of Two Serines. Nature 334 pp528-530

Carlson F. R., (1996) Olfaction in Drosophila from Odor to Behavior. TIG 12 (5) pp175-180

Carrington C. D. and Abou-Donia M. B., (1985) Axoplasmic Transport and Turnaround of Neurotoxic Esterase in Hen Sciatic Nerve. J. Neurochem. 44 pp616-621

Casida J. E., Eto M., and Baron R. L., (1961) Biological Activity of Tri o-cresyl Phosphate metabolite Nature 191 pp1396-1397 
Creighton T. E., (1993) Proteins Structure and Molecular Properties. W. H. Freeman and Company pp261-325

Chatel J.-M., Grassi J., Frobert Y., Massoulié J. and Vallette F.-M., (1993) Existence of an Inactive Pool of Acetylcholinesterase in Chicken Brain. Neurobiology 90 pp2476-2480

Clothier B. and Johnson M. K., (1974) Rapid Ageing of Neurotoxic Esterase after Inhibition by Di-isopropyl Phosphorofluoridate. Biochem. J. 177 pp549-558

Clark M., Lehrach H., Appel B., Eisen J., Johnson S., Marra M., Eddy S., Hillier L., Allen M., Bowles L., Dubuque T., Geisel G., Jost S., Kucaba T., Lacy,M., Le N., Lennon G., Martin J., Moore B., Schellenberg K., Steptoe M., Tan F., Theising B., White Y., Wylie T., Waterston R., Wilson R. (1997) WashU Zebrafish EST Project. Unpublished AC: AA494858

Clontech (1997) Marathon-Ready cDNA User manual (PT1156-1 version PR64097)

Chemnitius J. M., Haselmeyer K. H. and Zech R., (1984) Neurotoxic Esterase: Gel Filtration and Isoelectric Focusing of Carboxylesterases Solubilized from Hen Brain. Life Sci. 34 pp1119-1125

Dang C. V., Barrett J., Villagarcia M., Resar L. M. S., Kato G. J. and Fearon E. R., (1991) Intracellular Leucine Zipper Interactions Suggest C-Myc HeteroOligomerization. Molecular and Cellular Biology 11(2) pp954-962

Davies K. E. and Read A. P., (1988) Locating Genes. Molecular Basis of Inherited Disease Second Edition IRL press. Edited by D. Rickwood pp15-36

Davis C. S. and Richardson R., (1987) Neurotoxic Esterase: Characterisation of the Solubilized Enzyme and the Conditions for its Solubilization from Chicken Brain Microsomal Membranes with lonic, Zwitterionic, or non-ionic detergents. Biochem. Phamac 36 pp1393-1399

Devlin K., Churcher C., Barrell B. G. and Rajandream M. A., unpublished em:Z46729, sw: Q04958

Drapeau G. R. (1976) [38] Protease from Staphyloccus aureus. Methods in Enzymology. 45, pp469-475

Dry I. B. and Robinson S. P., (1994) Molecular cloning and characterisation of grape berry polyphenol oxidase Plant Molecular Biology 26 pp495-502

Edwards J. B. D. M., Ravassard P., Icard-Liepkalns C. and Mallet J. (1995) CDNA Cloning by RT-PCR. IRL press. PCR 2: A Practical Approach. Edited by McPherson M. J., Hames B. D. and Taylor G. R. pp89-118 
Escalera S. de la., Bockamp E.-O., Moya F., Piovant M. and Jiménez F., (1990) Characterization and gene cloning of neurotactin, a Drosophila transmembrane protein related to cholinesterases. EMBO Jounal 9 (11) pp3593-3601

Eto M., Casida J. E. and Eto T., (1962) Hydroxylation and Cyclization Reactions Involved in the Metabolism of Tri o-cresyl Phosphate. Biochem. Pharmacol. 11 pp337-352

Frohman M. A., (1990) RACE: Rapid Amplification of CDNA Ends. PCR Protocols: A guide to Methods and Applications. Academic Press, Inc. Edited by Innis M.A, Gelfand D. H., Sninsky J. J. and White T. J. pp28-38

Glynn P., Rüffer-Turner M. E., Read D. J., Wylie S. and Johnson M. K (1993) Molecular Characterisation of Neuropathy Target Esterase: Proteoolysis of the [3H]DFP-Labelled Polypeptide. Chem.-Biol. Interactions 87, 361-367

Glynn P., Read D. J., Guo R., Wylie S., and Johnson M. K., (1994) Synthesis and characterization of a biotinylated organophosphorus ester for detection and affinity purification of a brain serine esterase: neuropathy target esterase. Biochem. J. 301, 551-556

Glynn P., Holton J. L., Nolan C. C., Read D. J., Brown L., Hubbard A. and Cavanagh J. B., (1998) Neuropathy Target Esterase: Immunolocalization to Neuronal Cell Bodies and Axons. Neuroscience 83(1) pp295-302

Gnagey A. L., Forte M., and Rosenbery T. L., (1997) Isolation and Characterization of Acetylcholinesterase from Drosophila. J. Biol. Chem. 262(27) pp13290-13298

Goldenring J. R., Shen K. R., Vaughan H. D., and Modlin I. M., (1993) Identification of a Small GTP-binding Protein, Rab25, Experssed in the Gastrointestinal Mucosa, Kidney, and Lung. J. Biol. Chem. 268(25) pp1841918422

Heisenberg and Böhl (1979) Isolation of Brain Mutants of Drosophila by Histological Means. Z. Naturforsch 34 pp143-147

Hasan G., (1990) Molecular cloning of an Olfactory Gene from Drosphila Melanogaster. Proc. Natl. Acad. Sci. USA. 87 pp9037--9041

Hofmann K. and Stoffel W., (1993) TMbase- A Database of Membrane Spanning Protein Segments Biol. Chem. Hoppe-Seyler 347 pp166

Holmstedt B., (1963) Structure-activity Relationships of the Organophosphorus Anticholinesterase Agents. Cholinesterases and Anticholinesterase Agents. Berlin, Heidelberg: Springer. Edited by Koelle G. B. pp428-485

Henikoff and Henikoff, (1992) Amino Acid Substitution Matrices from Protein Blocks. Proc. Natl. Acad. Sci. USA 89; 10915-10919 
Hope I. A., Albertson D. G., Martinelli S. D., Lynch A. S., Sonnhammer E. and Durbin R., (1996) The C. elegans Expression Pattern Database: a Beginning TIG 12(9) pp370-371. URL:http://eatworms.swmed.edu/Worm_labs/Hope

Ichtchenko K., Hata Y., Nguyen T., Ullrich B., Messler M., Moomaw C. and Südhof T. C., (1995) Neuroligin 1: A Splice Site-Specific Ligand for b-Neurexins. Cell 81 pp435-443

Ichtchenko K., Nguyen T. and Südhof T. C., (1996) Structure, Alternative Splicing, and Neurexin Binding of Multiple Neuroligins. J. Biological Chemistry 271 (5) pp2676-2682

Inoue N., Fujishiro K., Mori K. and Matsuoka M., (1988) Triorthocresyl Phosphate Poisoning - A Review of Human Cases. J. UOEH 10 (4) pp433-442

Iwabuchi K., Li B., Bartel P. and Fields S. (1993) Use of the 2-Hybrid System to Identify the Domain of P53 Involved in Oligomerization. Oncogene 8(6), pp16931696

Johnson M. K., (1969a) A Phosphorylation Site in Brain and the Delayed Neurotoxic Effect of Some Organophosphorus Compounds. Biochem. J. 111 pp487-495

Johnson M. K., (1969b) The Delayed Neurotoxic Effect of Some Organophosphorus Compounds. Identification of the Phosphorylation Site as an Esterase. Biochem. J. 144 pp711-717

Johnson M. K., (1970) Organophosphorus and Other Inhibitors of Brain 'Neurotoxic esterase' and the Development of Delayed Neurotoxicity in Hens. Biochem. J. 120 pp523-531

Johnson M. K., (1975) Organophosphorus Esters Causing Delayed Neurotoxic Effects: Mechanism of Action and Structure/Activity Studies. Arch.Toxicol. 34 pp259-288

Johnson M. K. (1975b) Structure-Activity Relationships for Substrates and Inhibitors of Hen Brain Neurotoxic Esterase. Biochem. Pharmacol. 24 pp797-805

Johnson M. K., (1978) Improved Assay Of Neurotoxic Esterase for Screening Organophosphates for Delayed Neurotoxic Potential. Arch. Toxicol. 37 pp113115

Johnson M. K., (1982) The Target for Initiation of Delayed Neurotoxicity by Organophosphates Esters: Biochemical Studies and Toxicological Applications. Rev. Biochem. Toxicol Vol 4 New York: Elsevier. Edited by Hodgson E., Bend J. R. and Philpot R. M. pp141-212 
Johnson M. K. and Read D. J., (1987) The Influence of Chirality on the Delayed neuropathic potential of some organophosphate esters: delayed neuropathic and protective respectively are caused by the $L(-)$ and $D(+)$ isomers of EPN and its oxon. Toxicol. Appl. Pharmicol. 90 pp103-115

Johnson M. K., (1988) Sensitivity and Selectivity of Compounds Interacting with Neuropathy Target Esterase: Further Structure/Activity Studies. Biochem. Pharmacol.. 37 pp4094-4104

Johnson M. K., (1992) Molecular Events in Delayed Neuropathy: Experimental Aspects of Neuropathy Target Esterase. Clinical and Experimental Toxicology of Organophosphates and Carbamates. Butterworth Heinemann. Edited by Ballantyne B. and Marrs T. C. pp90-113

Johnson W. G., Murphy M., Murphy W. I. and Bloom A. D. (1987) Recessive congenital cerebellar disorder in a genetic isolate: CPD type VII Neurology(Abstract) 28 pp352-353.

Kidd K. K. and Ruano G. (1995) Optimizing PCR. PCR 2: A Practical Approach. Edited by McPherson M. J., Hames B. D. and Taylor G. R. pp1-22

Kozak M., (1986) Point mutations Define a Sequence Flanking the AUG Initiator Codon that Modulates Translation by Eukaryotic Ribosomes. Cell 44 pp283-292

Krejci E., Duval N., Chatonnet A., Vincens P., and Massoulie J., (1991) Cholinesterase-like domains in enzymes and structural proteins: Functional and evolutionary relationships and identification of a catalytically essential aspartic acid. Proc. Natl. Acad. Sci. USA 88, pp6647-6651

Kretzschmar D., Hasan G., Sharma S., Heisenberg M. and Benzer S., (1997) The swiss cheese mutant causes glial hyperwrapping and brain degeneration in Drosophila. J. Neurosci. 17 (19) pp7425-7432

Kwok S., Kellogg D. E., McKinney N., Spasic D., Goda L., Levenson C. and Sninsky J. J., (1990) Effects of Primer-Template Mismatches on the Polymerase Chain Reaction: Human Immunodeficency Virus Type 1 Model Studies. Nucleic Acids Research 18 (4) pp999-1005

Laemmli U. K., (1971) Cleavage of Structural Proteins During Assembly of Bacteriophage T4. Nature 227 pp680-685

Lamballe F., Klein R., and Barbacid M. (1991) trkC, a New Member of the trk Family of Tyrosine Protein Kinases Is a Receptor of Neurotrophin-3. Cell 66 pp967-979

Layer P. G., Weikert T. and Alber R., (1993) Cholinesterases Regulate Neurite Growth of Chick Nerve Cells In Vitro by Means of a Non-enzymic Mechanism. Cell Tissue Res 273 pp219-226 
Lee C. C. and Caskey C . T., (1990) CDNA Cloning using Degenerate Primers. PCR Protocols: A guide to Methods and Applications. Academic Press, Inc. Edited by Innis M.A, Gelfand D. H., Sninsky J. J. and White T. J. pp46-53

Lennon G. G., Auffray C., Polymeropoulos M. and Soares M. B., (1996) The I.M.A.G.E. Consortium: An Integrated Molecular Analysis of Genomes and their Expression. Genomics 33 pp151-152

Lotti M., (1992) The Pathogenesis of Organophosphate Polyneruopathy. Crit. Rev. Toxicol. 21(6) pp465-487

Lundberg K. S., Shoemaker D. D., Adams M. W. W., Short J. M., Sorge J. A., and Mathur E. J. (1991) High-fidelity Amplification Using a Thermostable DNA Polymerase Isolated from Pyrococcus furiosus. Gene 108 pp1-6

Lush, M. J., Li, Y., Read, D., Whillis, A. C. and Glynn, P. (1998) Neuropathy Target Esterase (NTE) and a homologous Drosophila neurodegenerativeassociated mutant protein contains a novel domain conserved from bacteria to man. Biochem. J. 332 pp1-4

Luque E. A., Lemanski L. F., and Dube D. K., (1994) Molecular Cloning, Sequencing and Expression of an Isoform of Cariac Alpha-tropomyosin from the Mexican Axoloti (Ambystoma Mexicanum). Biochem. and Biophys Research Communications 203(1) pp319-325

Mackay C. E., Hammock B. D. and Wilson B. W. (1996) Identification and Isolation of a $155 \mathrm{kDa}$ Protein with Neuropathy Target Esterase Activity. Fundamental and Applied Toxicology 30 pp23-30

McPherson M. J., Jones K. M. and Gurr S. J. (1991) PCR with highly degenerate primers. PCR: A Practical Approach. IRL press. Edited by McPherson M. J., Quirke P., and Taylor G. R. pp171-186

Meredith C. and Johnson M. K., (1989) Species Distribution of ParaoxonResistant Brain Polypeptides Radiolabelled with Diisopropyl Phosphorofluoridate ( $\left.\left.{ }^{3} \mathrm{H}\right] \mathrm{DiPF}\right)$ : Electrophoretic Assay for the Aged Polypeptide of [3H]DiPF-Labelled Neuropathy Target Esterase. J. Neurochem, 52, 1248-1252

Meredith C. and Johnson M. K., (1988) Neuropathy Target Esterase: Rates of Turnover In Vivo Following Covalent Inhibition with Phenyl Di-nPentylphosphinate. J. Neurochem. 51(4) pp1097-1101

Hirokawa T., Boon-Chieng S. and Mitaku S., (1998) SOSUI: Classification and Secondary Structure Prediction System for Membrane Proteins. in press, Bioinformatics, formerly known as CABIOS

Morgan J. P., and Penovich P., (1978) Jamaica Ginger paralysis. Archives Neurology 35 pp530-532 
Moretto A., Lotti M., Sabri M. I. and Spencer P. S., (1987) Progressive Deficit of Retrograde Axonal Transport is Associated with the Pathogenesis of di-n-butyl dichlorvos axonopathy. J. Neurochem 49 pp1515-1522

Nystuen A., Benke P. J., Merren J., Stone E., and Sheffield V. C., (1996) A Cerebellar Ataxia Locus Identified by DNA pooling to Search for Linkage Disequoilibrium in an Isolated Population from the Cayman Islands. Human Mol. Genet. 5 (4) pp525-531

Ochman H., Medhora M. M., Garza D., and Hartl D. L. (1990) Amplification of Flanking Sequences by Inverse PCR. PCR Protocols: A guide to Methods and Applications. Academic Press, Inc. Edited by Innis M.A, Gelfand D. H., Sninsky J. J. and White T. J. pp219-227

Olender E. H. and Simoni R. D., (1992) The intracellular Targeting and membrane Topology of 3-Hydroxy-3-methyl Glutaryl-CoA Reductase. J. Biol. Chem. 267 pp4233-4235

Olson P. F., Fessler L. I., Nelson R. E., Sterne R. E., Campbell A. G. and Fessler J. H., (1990) Glutactin, a Novel Drosophila Basement Membranerelated Glycoprotein with Sequence Similarity to Serine Esterases. EMBO J. 9(4) pp1219-1227

OMIM (1997), Online Mendelian Inheritance in Man. Center for Medical Genetics, Johns Hopkins University (Baltimore, MD) and National Center for Biotechnology Information, National Library of Medicine (Bethesda, MD), 1997. World Wide Web URL: http://www.ncbi.nlm.nih.gov/omim/

Paterson J. and O'Hare K., (1991) Structure and Transcription of the Singed Locus of Drosophila Melanogaster. Genetics 129 pp1073-1084

Poulsen E. and Aldridge W. N., (1964) Studies On Esterases In The Chicken Central Nervous System. Biochem. J. 90 pp182-189

Pope C. N. and Padillia S. S., (1989) Chromatographic characterization of neurotoxic esterase. Biochem. Pharmacol. 38 pp181-188

Promega (1996) Promega Protocols and Applicaton Guide $3 r^{d}$ Edition Promega, pp49-51

QIAGEN (1997) QIAquick Spin Handbook. QIAGEN

Rice G. C., McCabe S., Pennica D. P., Woronicz J., Borree J. and Goeddel D. V. (1990) Expression Cloning by Transient Expression Utilising Fluorescence Activated Cell Sorting. DNA and Protein Engineering Techniques 2 (1) pp18-22

Rüffer-Turner M. E., Read D. J., and Johnson M. K., (1992) Purification of Neuropathy Target Esterase from Avian Brain After Prelavelling with [3H]Disopropyl Phosphorpfluoridate. J. Neurochem. 58 (1) pp135-141 
SambrookJ., Fritsh E. F. and Maniatis T., (1989a) Molecular Cloning, A Laboratory Manual ( ${ }^{\text {nd }}$ edition) Cold Spring Harbor Laborotory Press

SambrookJ., Fritsh E. F. and Maniatis T., (1989b) Molecular Cloning, A Laboratory Manual( $2^{\text {nd }}$ edition) Cold Spring Harbor Laborotory Press pp12.4

Schmidt H., Dresselhaus T., Buck F. and Heinz E., (1994) Purification and PCR-based CDNA Cloning of a Plastidial n-5 Desaturase. Plant Molecular Biology 26 pp631-642

Schmidt J. V., Carver L. A., and Bradfield C. A. (1993) Molecular characterization of the Murind Ahr Gene. J. Biol. Chem. 268, 22203-22209

Schuler G.D., Boguski M. S., Stewart E. A., Stein L. D., Gyapay G., Rice K., White R. E., Rodriguez-Tomé P., Aggarwal A., Bajorek E., Bentolila S., Birren B. B., Butler A., Castle A. B., Chiannilkulchai N., Chu A., Clee C., Cowles S., Day P. J. R., Dibling T., Drouot N., Dunham I., Duprat S., East C., Edwards C., Fan J.-B., Fang N., Fizames C., Garrett C., Green L., Hadley D., Ma J., MacGilvery A., Mader C., Maratukulam A., Matise T. C., Mckusick K. B., Morissette J., Mungall A., Muselet D., Nusbaum H. C., Page D. C., Peck S., Perkins S., Piercy M., Qin F., Quackenbush J., Ranby S., Reif T., Rozen S., Sanders C., She X., Silva J., Slonim D. K., Soderlund C., Sun W.-L., Tabar P., Thangarajh T., Vega-Czarny N., Vollrath D., Voyticky S., Wilmer T., Wu X., Adams M. D., Auffray C., Walter N. A. R., Brandon R., Dehejia A., Goodfellow P. N., Houlgatte R., Hudson Jr J. R., Ide S. E., lorio K. R., Lee W. Y., Seki N., Nagase T., Ishikawa K., Nomura N., Phillips C., Polymeropoulos M. H., Sandusky M., Schmitt K., Berry R., Swanson K., Torres R., Venter J. C., Sikela J. M., Beckmann J. S., Weissenbach J., Myers R. M., Cox D. R., James M. R., Bentley D., Deloukas P., Lander E. S. and Hudson T. J. (1996) A Gene Map of the Human Genome. Science 274 pp540-546

Schwartz, R. M. and Dayhoff, M. O. [1979] Atlas of Protein Sequence and Structure. National Biomedical Research Foundation, Washington D.C. Edited by Dayhoff, M. O. pp353-358

Silver J., (1991) Inverse Polymerase Chain Reaction. PCR: A Practical Approach. IRL press. Edited by McPherson M. J., Quirke P., and Taylor G. R. pp 137-146

Simmonds R. J., 1992 Chemistry of Biomolecules An Introduction. Royal Society of Chemistry. pp94-95

Smith M.I., Elvove E. and Frazier W. H., (1930) The pharmacological action of certain phenol esters with special reference to the etiology of so-called ginger paralysis Public Health Reports, 45 (42) pp2509-2524

Stratagene, (1997) pCR-Script Amp SK(+) Cloning Kit. Instruction Manual (Revision \#076004) 
Taylor G. R., (1991 Polymerase Chain Reaction: Basic Priniples and Automation. PCR: A Practical Approach. IRL press. Edited by McPherson M. J., Quirke P., and Taylor G. R. pp1-14

Thomas T. C., Székács A., Hammock B. D., Wilson B. W. and McNamee M. G. (1990) Characterization of Neuropathy Target Esterase using Trifluromethylketones. Biochem. Pharmacol. 257 pp106-116

Thomas T. C., Székács A., Hammock B. D., Wilson B. W. and McNamee M. G. (1993) Affinity Chromatography of Neuropathy Target Esterase. ChemicoBiological Interactions 87 No. 1-3 pp347-360

Tindall, K. R., and Kunkel T. A. (1988) Fidelity of DNA synthesis by the Thermus aquaticus DNA polymerase. Biochemistry 27; 6008-6013

Wicking C. and Williamson B. (1991) From Linked Marker to Gene. TIG 7 (9) pp288-293

Williams D. G. and Johnson M. K. (1981) Gel Electrophoretic Identification of Hen Brain Neurotoxic Esterase, Labelled with Tritiated Diisopropyl Phosphorofluoridate. Biochem J. 199 pp323-333

Wilson R., Ainscough R., Anderson K., Baynes C., Berks M., Bonfield J., Burton J., Connell M., Copsey T., Cooper J., Coulson A., Craxton M., Dear S., Du Z., Durbin R., Favello A., Fraser A., Fulton L., Gardner A., Green P., Hawkins T., Hillier L., Jier M., Johnston L., Jones M., Kershaw J., Kirsten J., Laisster N., Latreille P., Lightning J., Lloyd C., Mortimore B., O'callaghan M., Parsons J., Percy C., Rifken L., Roopra A., Saunders D., Shownkeen R., Sims M., Smaldon N., Smith A., Smith M., Sonnhammer E., Staden R., Sulston J., Thierry-Mieg J., Thomas K., Vaudin M., Vaughan K., Waterson R., Watson A., Weinstock L., Wilkinson-Sproat J., Wohldman P. (1994). $2.2 \mathrm{Mb}$ of Contiguous Nucleotide Sequence from Chromosome III of C. elegans. Nature 368 32-38

Yoshida M., Tomizawa M., Wu S.-Y., Quistad G. B. and Casida (1995) Neuropathy Target Esterase of Hen Brain: Active Site Reactions with 2-[octyl${ }^{3} \mathrm{H}$ ]Octyl-4H-1,3,2-Benzodioxaphosphorin 2-Oxide and 2-Octyl-4H-1,3,2-[aryl- ${ }^{3} \mathrm{H}$ ] Benzodioxaphosphorin 2-Oxide. J. Neurochem. 64(4) pp 1680-1687 